\title{
TARGETING ANTIGEN PRESENTING CELLS TO TREAT AUTOIMMUNE INFLAMMATION
}

\author{
by \\ Aras Toker \\ Malaghan Institute of Medical Research \\ Wellington, New Zealand
}

\begin{abstract}
a thesis
submitted to the Victoria University of Wellington in fulfilment of the requirements for the degree of Master of Science in Cell \& Molecular Bioscience
\end{abstract}

May 2009 


\section{Abstract}

Glatiramer acetate (GA) is approved for the treatment of relapsing-remitting multiple sclerosis (MS), and can suppress experimental autoimmune encephalomyelitis (EAE), a murine model of human MS. GA treatment is associated with the induction of anti-inflammatory $\mathrm{T}_{\mathrm{H}} 2$ responses and with the antigen specific expansion of regulatory $\mathrm{T}$ cells that counteract or inhibit pathogenic events in MS and EAE. These $\mathrm{T}$ cell mediated mechanisms of protection are considered to be a result of modulation of antigen presenting cells (APCs) by GA, rather than direct effects on T cells. However, it is unknown if GA preferentially targets a specific APC subset or can act through multiple APCs . In addition, GA-modulated innate cells may also exhibit direct antigen non-specific suppression of autoreactive cells.

One objective of this study was to identify the target cell population of GA and to assess the potential of the target cells to antigen non-specifically suppress immune responses. Fluorophor-labelled GA bound to monocytes after intravenous injections, suggesting that monocytes may be the primary target of GA In addition, intravenous GA treatment enhanced the intrinsic ability of monocytes to suppress $\mathrm{T}$ cell proliferation, both and . The findings of this study therefore suggest that GA-induced monocytes may contribute to GA therapy through direct mechanisms of antigen non-specific $\mathrm{T}$ cell immunosuppression.

A further objective of this work was to investigate the potential of an drug targeting approach. This approach was hypothesised to increase the uptake of GA by the target cells and substantially improve GA treatment through antigen specific mechanisms such as induction of $\mathrm{T}_{\mathrm{H}} 2$ or regulatory $\mathrm{T}$ cells. Targeting antigens to professional APCs with an anti-MHC class II antibody resulted in significantly enhanced T cell proliferation

. However, no EAE suppression occurred when GA was targeted to MHC class II . In addition, targeting GA specifically to monocytes also failed to suppress EAE.

These findings suggest that GA treatment may selectively modulate monocytes to enhance their ability to inhibit autoreactive T cells, which could be part of the mechanism by which GA ameliorates MS. Targeting GA to a specific cell type may not be a powerful approach to improve treatment, because increased proliferation of GA specific $\mathrm{T}$ cells is not sufficient for disease suppression, and conjugation to antibodies may functionally reduce GA to a mere antigen devoid of immunomodulatory capacity. 


\section{Table of Contents}

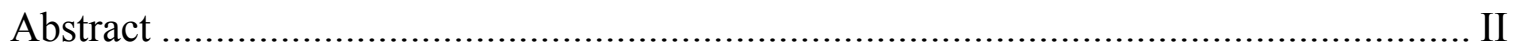

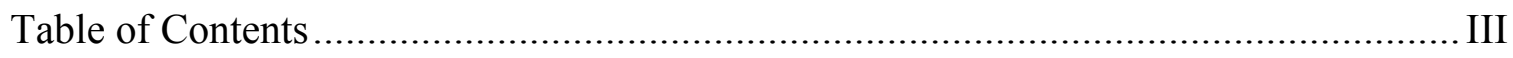

List of Figures and Tables .................................................................................. VII

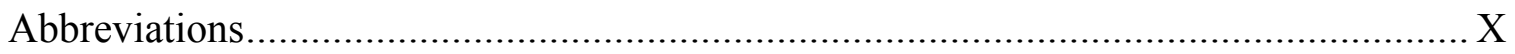

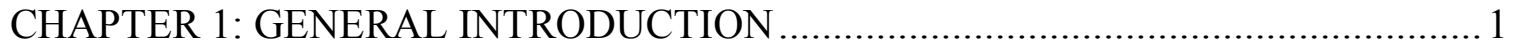

1.1 Principles of Innate and Adaptive Immunity ...................................................... 2

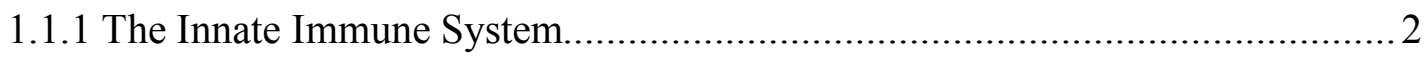

1.1.2 The Adaptive Immune System .............................................................. 3

1.2 Antigen Presentation to T Cells .................................................................... 4

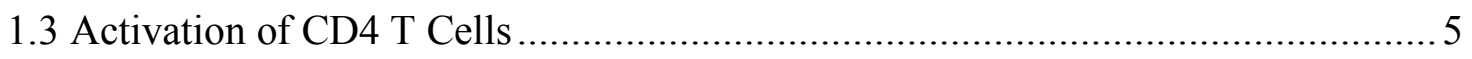

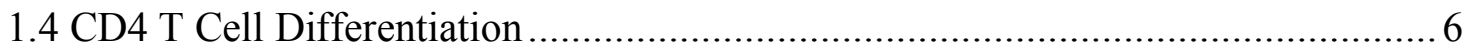

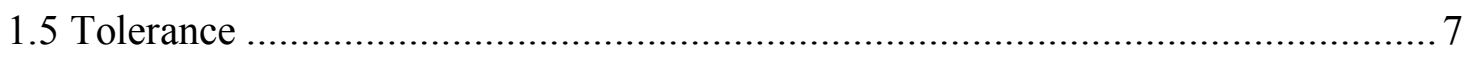

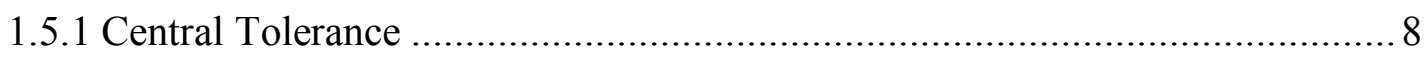

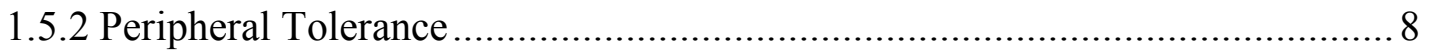

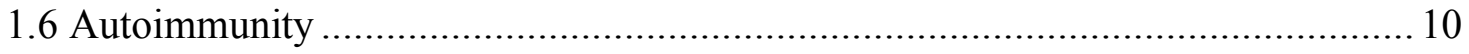

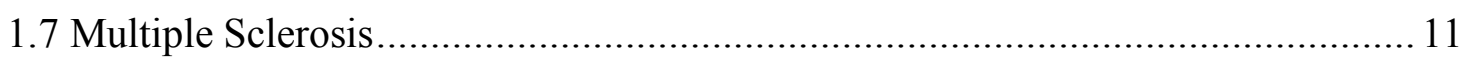

1.7.1 Statistics and Epidemiology …………...................................................... 11

1.7.2 Murine EAE as a Model of Human MS..................................................... 12

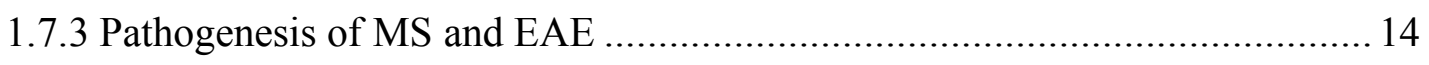

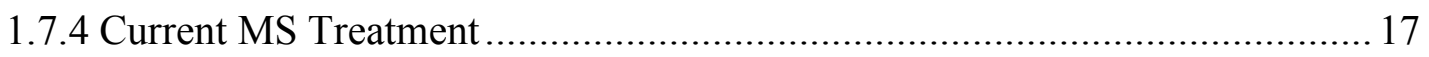

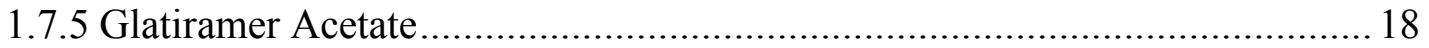

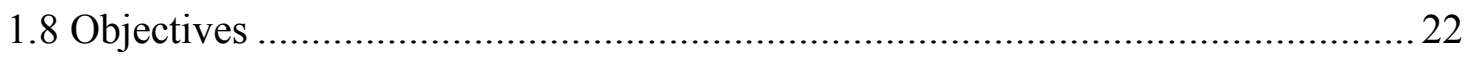

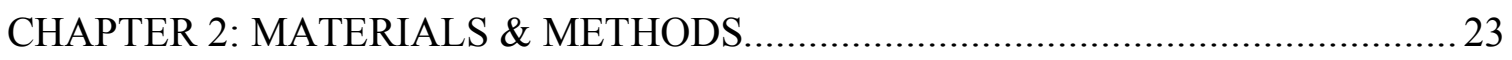

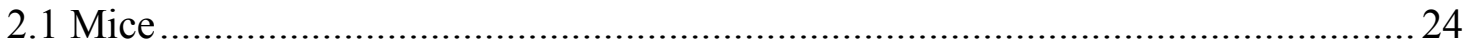

2.1.1 Maintenance and Ethic Approvals...........................................................2 24

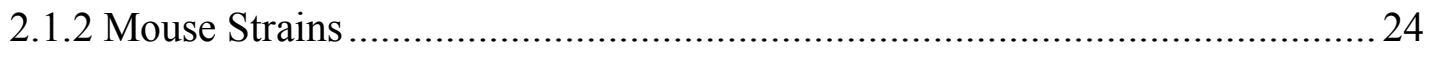

2.1.3 Genotyping of TCR Transgenic Mouse Strains …………………………...25

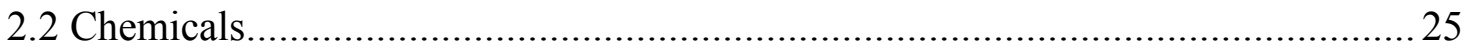

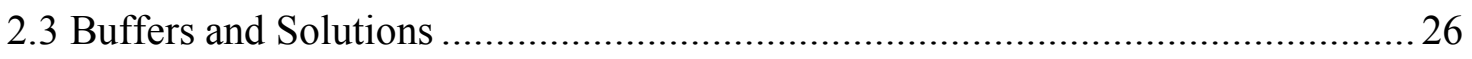

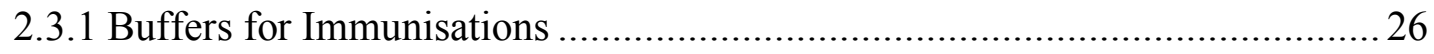

2.3.2 Buffers and Media for Cell Preparation and Tissue Culture...........................26 


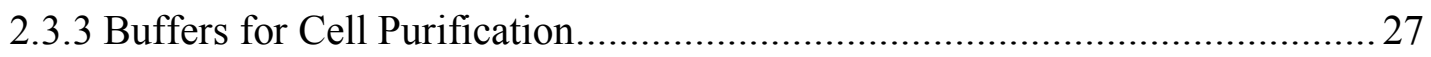

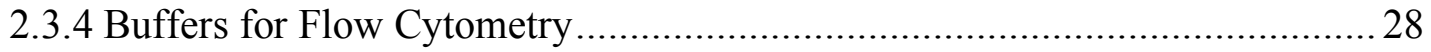

2.3.5 Solutions for Preparation of $\mathrm{Cl}_{2} \mathrm{MDP}$ Liposomes ...................................... 28

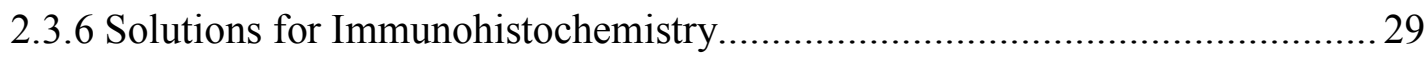

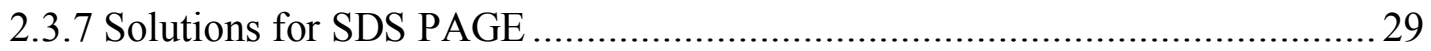

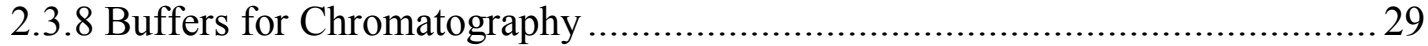

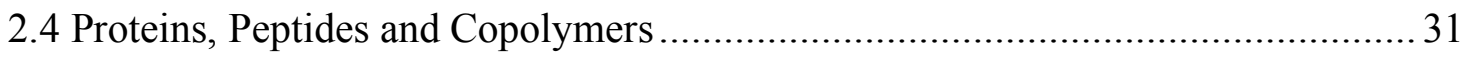

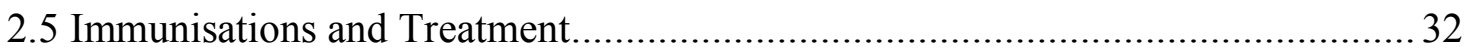

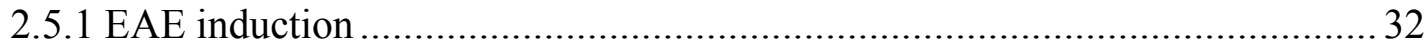

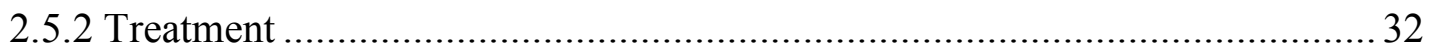

2.5.3 Immunisations to Prime Cells for Proliferation Assays or to Study Lymphoid

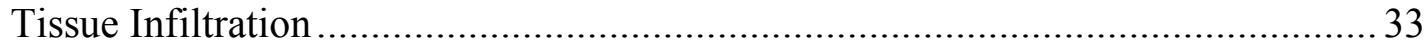

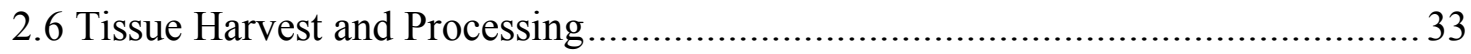

2.6.1 Preparation of Cells from Spleens and Lymph Nodes..................................... 33

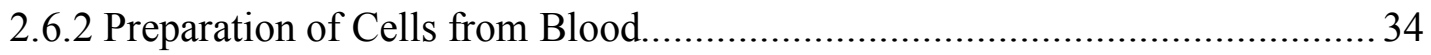

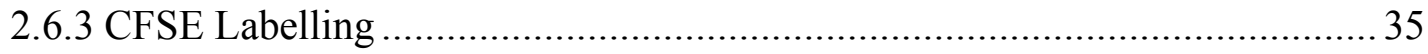

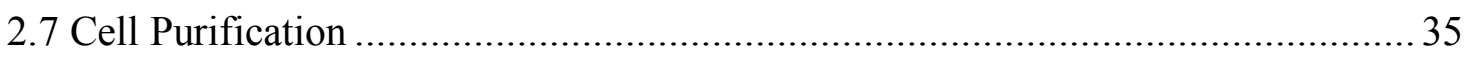

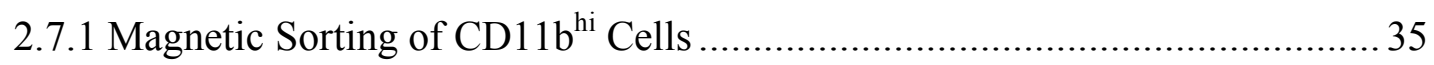

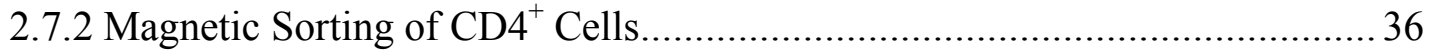

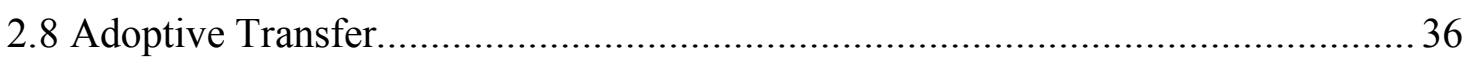

2.9 Proliferation and Suppression Assays ............................................................... 37

2.9.1 Antigen Specific Proliferation Assays................................................. 37

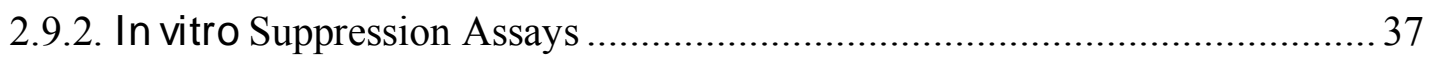

2.9.3 Antigen specific Proliferation Assays ................................................... 38

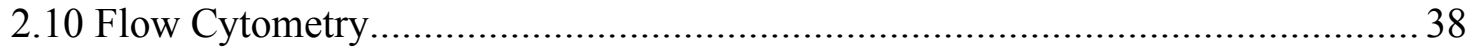

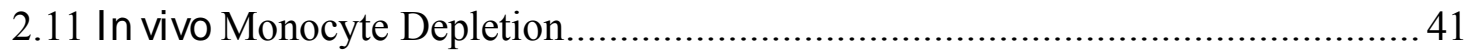

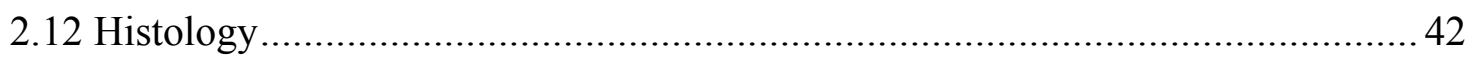

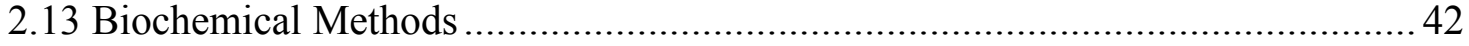

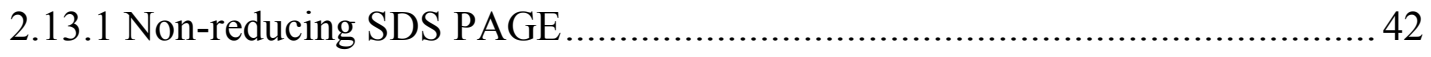

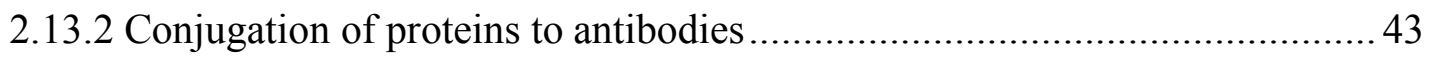

2.13.3 Conjugation of GA to Superantigens......................................................... 43

2.13.4 Fast protein liquid chromatography (FPLC) ............................................... 44

2.13.5 Fluorophor Labelling of Antibodies and Proteins ........................................ 46 
2.14 Statistical Analysis

CHAPTER 3: TARGETING GLATIRAMER ACETATE TO ANTIGEN PRESENTING

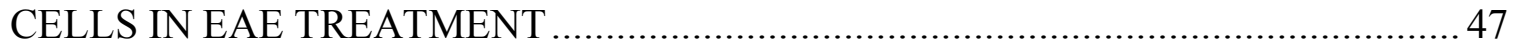

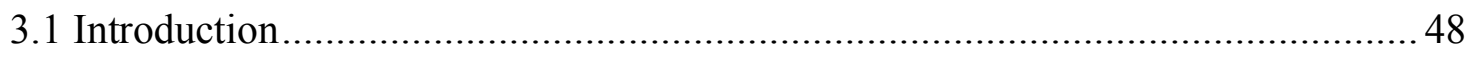

3.1.1 The MHCII Antigen Presentation Pathway ............................................. 48

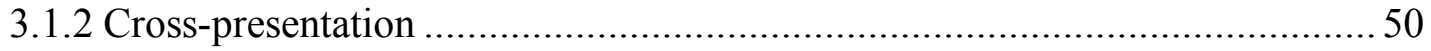

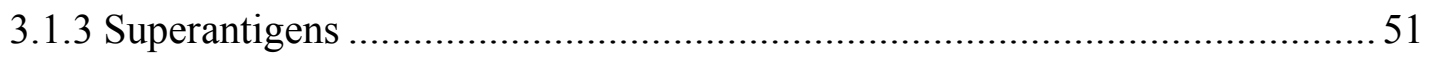

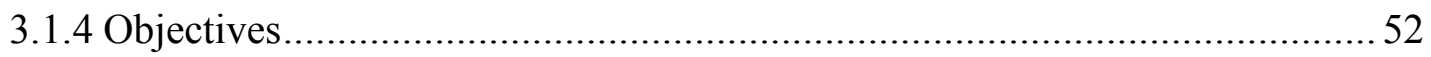

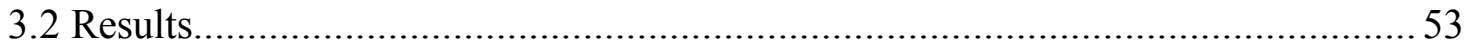

3.2.1 Establishment of a GA Treatment Model of EAE Suppression .................... 53

3.2.1.1 Pre-treatment with GA in PBS does not suppress EAE ........................53

3.2.1.2 Co-Immunisation with $\mathrm{MOG}_{35-55}$ and GA Suppresses EAE .................. 54

3.2.1.3 Mannitol is not Required for Effective Co-Immunisation Treatment ......57

3.2.1.4 GA does not Suppress EAE in $\mathrm{MOG}_{35-55}$ TCR Transgenic Mice.............58

3.2.2 Antigen Targeting to MHCII with a Specific Antibody $\quad$.....................6 60

3.2.2.1 Conjugation of MOG and OVA Proteins to an anti-MHCII Antibody..... 60

3.2.2.2 Targeting MOG and OVA to MHCII Enhances CD4 T Cell Proliferation

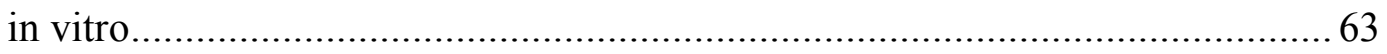

3.2.2.3 Enhanced CD4 T Cell Proliferation is Independent of Fc $\gamma \mathrm{R}$ Mediated

Uptake of M5/114-Antigen Conjugates..................................................... 66

3.2.2.4 Targeting OVA to MHCII enhances CD8 T cell Proliferation in vitro .... 67

3.2.3 Antigen Targeting to MHCII with a Modified Superantigen $\quad$.............6 68

3.2.4 Targeting GA to MHCII Increases T Cell Proliferation $\quad$.....................6 69

3.2.5 Targeting GA to MHCII does not suppress EAE...................................... 72

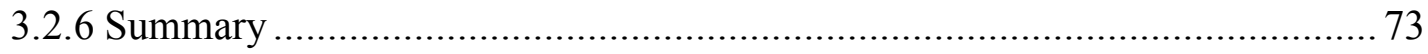

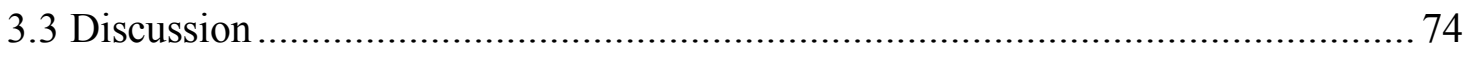

3.3.1 Establishment of a GA Treatment Model in C57BL/6J Mice....................... 74

3.3.2 GA does not Protect TCR Transgenic Mice from EAE.............................. 75

3.3.3 Targeting Antigens to MHCII Enhances T Cell Proliferation $\quad$............ 76

3.3.4 Targeting GA to MHCII in EAE Treatment ........................................... 78

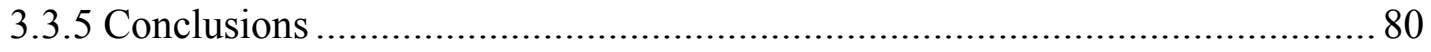


CHAPTER 4: TARGETING GLATIRAMER ACETATE TO MONOCYTES IN EAE

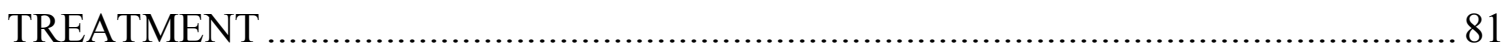

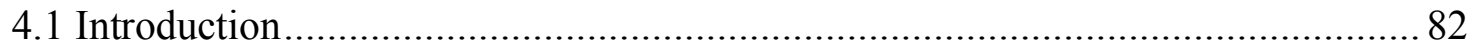

4.1.1 Monocytes in Innate and Adaptive Immunity ......................................... 82

4.1.1.1 Monocyte Subpopulations ........................................................ 82

4.1.1.2 Differential Activation of Monocytes and Macrophages ....................... 84

4.1.2 Myeloid-Derived Suppressor Cells.................................................... 85

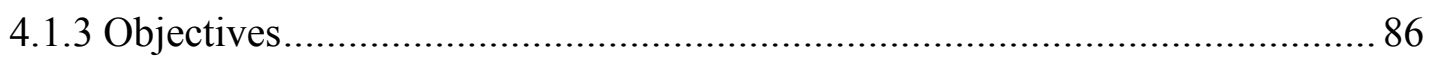

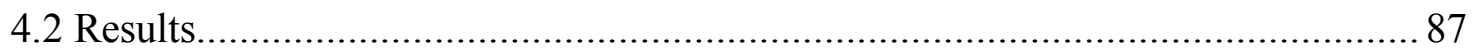

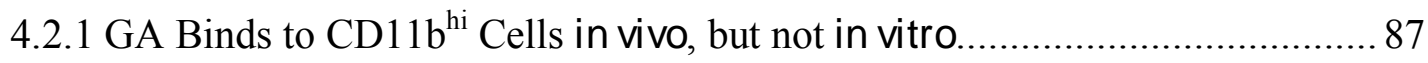

4.2.1.1 GA ${ }^{+}$Cells in the Blood Show Monocyte Cell Surface Phenotype ........... 91

4.2.1.2 Time-course of Monocyte Staining by GA ........................................ 93

4.2.2 Intravenous GA Treatment Enhances the Suppressive Ability of Monocytes. 94

4.2.3 Effects of GA on Monocytes cannot be Detected in Draining Lymph Nodes. 98 4.2.4 GA Treatment does not Impair T Cell Proliferation in EAE ....................... 101

4.2.5 Temporary Monocyte Depletion does not Impair GA Treatment of EAE .... 104

4.2.6 Targeting GA to CD11b does not Suppress EAE at Low Dose................... 105

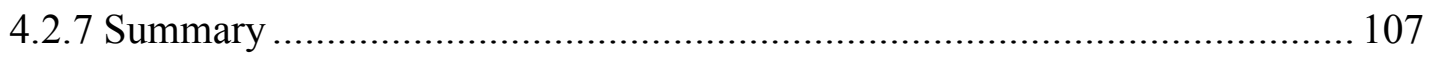

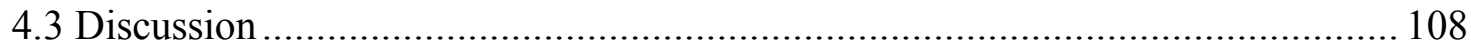

4.3.1 GA Binding to Blood Monocytes $\quad$............................................... 108

4.3.2 Does GA Treatment Affect Monocytes in the Draining Lymph Node?........ 110

4.3.3 T Cell Suppression by GA-Modulated Monocytes ................................. 111

4.3.4 Targeting GA to Monocytes in EAE Treatment ................................... 113

4.3.5 The Role of Trypsinogen and DM SMEZ2 ........................................... 114

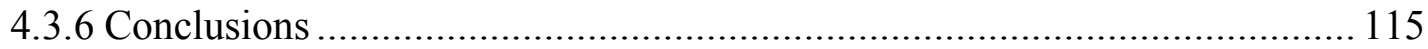

CHAPTER 5: GENERAL DISCUSSION ............................................................ 117

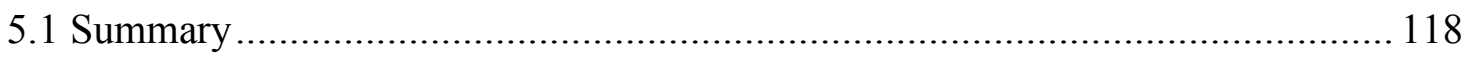

5.2 Antigen Targeting Strategies in Disease Treatment....................................... 119

5.2.1 MHCII as an Antigen Target.......................................................... 119

5.2.2 Does Targeting GA to Cell Surface Molecules Have Therapeutic Potential?120

5.3 Glatiramer Acetate: Update on Mechanism of Action ................................... 122

5.3.1 How Important is Direct Binding to MHCII? ........................................ 122

5.3.2 Divergent T cell Mediated Mechanisms of Suppression ........................... 123 
5.3.3 Possible Contribution of Multiple APC Subsets to GA Therapy................. 124

5.3.4 Possible Role for Suppressive Monocytes in GA Treatment...................... 124

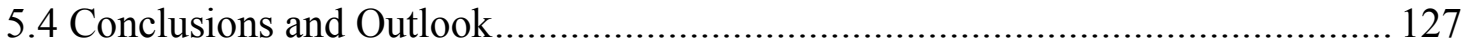

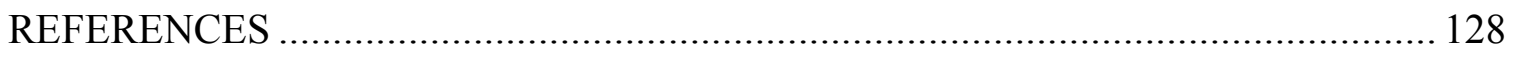

APPENDIX 1: SUPPLEMENTARY FIGURES AND TABLES ............................ 138

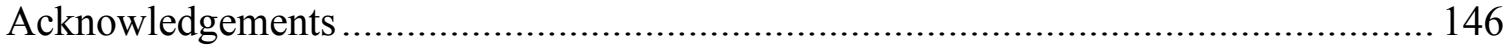




\section{List of Figures and Tables}

Figure 1.1: Mechanism of Action of Glatiramer Acetate (GA) in EAE Suppression. ..... 21

Table 2.1: Antibodies used for flow cytometry.............................................................. 39

Figure 2.1: Gating on total or mononuclear cells in flow cytometry............................. 40

Figure 2.2: Gating based on cell surface marker expression in flow cytometry. ............. 41

Figure 3.1: Overview on the MHCII antigen presentation pathway................................ 49

Figure 3.2: Pre-treatment with GA in PBS does not suppress EAE............................... 53

Figure 3.3: Co-immunisation with $\mathrm{MOG}_{35-55}$ and GA in CFA suppresses EAE.............. 54

Figure 3.4: GA treatment prevents cellular infiltration of the spinal cord....................... 55

Figure 3.5: Determination of the optimal dose of GA for efficient EAE suppression. .... 56

Figure 3.6: Co-immunisation treatment is superior to GA treatment in the neck. ........... 57

Figure 3.7: Therapeutic effect of GA does not depend on the presence of mannitol. ...... 58

Figure 3.8: GA does not suppress EAE in $\mathrm{MOG}_{35-55} \mathrm{TCR}$ transgenic mice. ....................59

Table 3.1: Optimisation of the conjugation of M5/114 to MOG...................................... 61

Figure 3.9: Optimisation of the conjugation of MOG and OVA proteins to M5/114...... 62

Table 3.2: Purification of M5/114-antigen conjugates by chromatography. .................... 63

Figure 3.10: Targeting MOG to MHCII enhances CD4 T cell proliferation _......64

Figure 3.11: Targeting OVA to MHCII enhances CD4 T cell proliferation $\quad$........65

Figure 3.12: Increased CD4 T cell proliferation to M5/114-MOG is not diminished by

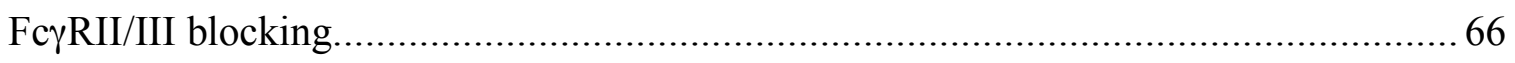

Figure 3.13: Targeting OVA to MHCII enhances CD8 T cell proliferation $\quad$........67

Figure 3.14: Targeting MOG and OVA proteins to MHCII with a modified SAg results in enhanced CD4 $\mathrm{T}$ cell proliferation

Figure 3.15: Targeting GA to MHCII results in enhanced T cell proliferation................. 70

Figure 3.16: Targeting GA to MHCII with a monoclonal antibody does not suppress

EAE

Figure 3.17: Targeting GA to MHCII with a modified SAg does not suppress EAE. ..... 73

Figure 4.1: GA binds to a CD11 $\mathrm{b}^{\text {hi }}$ cell population after intravenous injection............... 88

Figure 4.2: GA staining of $\mathrm{CD} 11 \mathrm{~b}^{\text {hi }}$ cells with different fluorophors.............................. 89

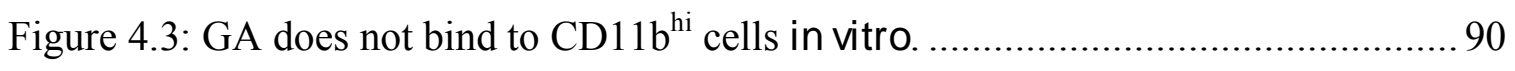

Figure 4.4: $\mathrm{GA}^{+}$cells in the blood show monocyte cell surface phenotype...................... 91

Figure 4.5: Binding of GA to CD1 $1 \mathrm{~b}^{\mathrm{hi}}$ cells is independent of MHCII.......................... 92 
Figure 4.6: GA staining of blood monocytes is stable over 18 hours.

Figure 4.7: GA enhances the ability of monocytes to suppress polyclonal $\mathrm{T}$ cell

proliferation.

Figure 4.8: GA treated and naïve monocytes equally suppress $\mathrm{MOG}_{35-55}$ specific $\mathrm{T}$ cell

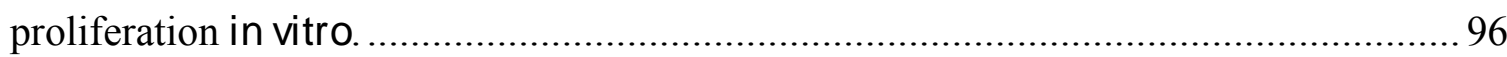

Figure 4.9: Intravenous GA treatment suppresses CD4 T cell proliferation $\quad$........97

Figure 4.10: GA-FITC cannot be detected in lymph nodes after immunisation. .............98

Figure 4.11: The percentage of CD11 bi ${ }^{\text {hi }}$ cells in the draining lymph node does not change

after immunisation.

Figure 4.12: $\mathrm{CD} 11 \mathrm{~b}^{\text {hi }} \mathrm{Ly}_{6 \mathrm{G}}^{+}$granulocytes accumulate in spleen and blood after immunisation with CFA.

Figure 4.13: Adoptively transferred monocytes cannot be detected in draining lymph

nodes of immunised recipients.

Figure 4.14: Co-immunisation with GA does not impair $\mathrm{MOG}_{35-55}$ specific $\mathrm{T}$ cell

proliferation. 102

Figure 4.15: Co-immunisation treatment with $\mathrm{GA}$ does not suppress $\mathrm{MOG}_{35-55}$ specific $\mathrm{T}$ cell proliferation in EAE.

Figure 4.16: Temporary monocyte depletion does not impair GA treatment of EAE... 104

Figure 4.17: Intravenous GA treatment does not suppress EAE.............................. 105

Figure 4.18: Targeting GA to CD11b does not suppress EAE................................ 106

Figure 5.1: Proposed updates on the mechanism of action of GA in EAE.................. 126 


$\begin{array}{ll}\text { Abbreviations } \\ \text { APC } & \text { Antigen presenting cell } \\ \text { APC } & \text { Allophycocyanin } \\ \text { APL } & \text { Altered peptide ligand } \\ \text { BBB } & \text { Blood-brain barrier } \\ \text { BDNF } & \text { Brain-derived neurotrophic factor } \\ \text { CCR2 } & \text { CC-chemokine receptor 2 } \\ \text { CFA } & \text { Complete Freund's adjuvant } \\ \text { CFSE } & \text { Carboxyfluorescein succinimidyl ester } \\ \text { CNS } & \text { Central nervous system } \\ \text { CTL } & \text { Cytotoxic T lymphocyte } \\ \text { DMF } & \text { Dimethylformamide } \\ \text { DMSO } & \text { Dimethylsulfoxide } \\ \text { DC } & \text { Dendritic cell } \\ \text { dH }{ }_{2} \mathrm{O} & \text { distilled water } \\ \text { EAE } & \text { Experimental autoimmune encephalomyelitis } \\ \text { EDTA } & \text { Ethylene diamine tetra acetate } \\ \text { ER } & \text { Endoplasmic reticulum } \\ \text { FACS } & \text { Fluorescence-activated cell sorting } \\ \text { FCS } & \text { Foetal calf serum } \\ \text { FITC } & \text { Fluorescein isothiocyanate } \\ \text { FPLC } & \text { Fast protein liquid chromatography } \\ \text { GA } & \text { Glatiramer acetate } \\ \text { Gr-1 } & \text { Granulocyte antigen 1 } \\ \text { IFA } & \text { Incomplete Freund's adjuvant } \\ \text { IFN } & \text { Interferon } \\ \text { Ig } & \text { Immunoglobulin } \\ \text { IL } & \text { Interleukin } \\ \text { iNOS } & \text { Inducible nitric oxide synthase } \\ \text { LPS } & \text { Lipopolysaccharide } \\ \text { Mac-1 } & \text { Membrane activating complex 1 } \\ \text { MBP } & \text { Myelin basic protein } \\ & \end{array}$




$\begin{array}{ll}\text { MCP-1 } & \text { Monocyte chemoattractant protein-1 } \\ \text { MDSC } & \text { Myeloid-derived suppressor cell } \\ \text { MFI } & \text { Mean fluorescence index } \\ \text { MHC } & \text { Major histocompatibility complex } \\ \text { MOG } & \text { Myelin oligodendrocyte glycoprotein } \\ \text { MMP } & \text { Matrix metalloprotease } \\ \text { MRI } & \text { Magnetic resonance imaging } \\ \text { MS } & \text { Multiple sclerosis } \\ \text { MSCH } & \text { Mouse spinal cord homogenate } \\ \text { PBS } & \text { Phosphate-buffered saline } \\ \text { PBMC } & \text { Peripheral blood mononuclear cell } \\ \text { PE } & \text { Phycoerythrin } \\ \text { PerCP } & \text { Peridinin chlorophyll protein } \\ \text { PLP } & \text { Proteolipid protein } \\ \text { PPMS } & \text { Primary progressive multiple sclerosis } \\ \text { PRR } & \text { Pattern recognition receptor } \\ \text { ROS } & \text { Reactive oxygen species } \\ \text { rpm } & \text { Revolutions per minute } \\ \text { RRMS } & \text { Relapsing-remitting multiple sclerosis } \\ \text { RT } & \text { Room temperature } \\ \text { SA } & \text { Streptavidin } \\ \text { SDS-PAGE } & \text { Sodium dodecyl sulfate polyacrylamide gel electrophoresis } \\ \text { SMEZ } & \text { Streptococcal mitogenic exotoxin Z } \\ \text { SPMS } & \text { Secondary progressive multiple sclerosis } \\ \text { STAT } & \text { signal transducer and activator of transcription } \\ \text { TAP } & \text { Transporter associated with antigen processing } \\ \text { TCR } & \text { T cell receptor } \\ \text { TGF } & \text { Transforming growth factor } \\ \text { TH } & \text { T helper } \\ \text { TLR } & \text { Toll-like receptor } \\ \text { TNF } & \text { Tumour necrosis factor } \\ \text { T-reg } & \text { Regulatory T cell } \\ & \end{array}$




\section{CHAPTER 1:}

\section{GENERAL INTRODUCTION}


The main function of the immune system is to protect the host against harmful pathogens, through a combination of innate and adaptive immune responses. The innate immune system provides the first line of defence against pathogens. Innate immune responses occur rapidly, are not specific to a particular antigen and do not confer immunological memory. In contrast, adaptive immune responses are antigen specific and show delayed kinetics in comparison to innate immunity. In addition, adaptive immunity results in the generation of immunological memory, which confers improved and accelerated immune responses against subsequent challenges with the same pathogen.

The ability to provide effective antigen specific adaptive immune responses requires a vast and diverse lymphocyte repertoire. One of the drawbacks of this lymphocyte diversity is the presence of self-reactive clones among lymphocytes. In normal conditions, self-reactivity is avoided by multiple mechanisms that maintain selftolerance. However, when one or several of these regulatory mechanisms fail, autoimmune disorders such as multiple sclerosis (MS) can develop.

This general introduction will briefly summarise the innate and adaptive arms of the immune system, and review mechanisms of tolerance and development of autoimmunity. Probable causes, pathogenesis and current treatment of MS will be introduced, with particular focus on treatment with the current MS therapeutic glatiramer acetate (GA).

\subsection{Principles of Innate and Adaptive Immunity}

\subsubsection{The Innate Immune System}

The innate immune system forms the first line of defence against invading pathogens. Components of the innate immune system include physical barriers such as the skin, mucosal surfaces and epithelia; chemical mediators such as complement, and a variety of immune cells with specialised functions (1).

Cells of the innate immune system express pattern recognition receptors (PRRs) that recognise conserved structures on pathogens and trigger the initial cell-mediated innate response (1). The best-defined pattern recognition receptors are Toll-like receptors (TLRs), which recognise bacterial or viral components such as lipopolysaccharide (LPS)

of the gram-negative bacterial cell wall or virus-derived double stranded RNA (2). TLR signalling results in the activation of transcription factors that promote the expression of 
inflammatory cytokines, chemokines or other inflammatory mediators that initiate an immediate innate immune response against the pathogen (2).

An important cellular component of the innate immune system are phagocytes that can recognise pathogens through PRRs, followed by the internalisation and destruction of the pathogen. Monocytes and macrophages are mononuclear phagocytes that are found in the blood or tissue, respectively (3). Neutrophils are polymorphonuclear phagocytes that are found in the blood and tissue. In addition to phagocytosis, neutrophils also contribute to the elimination of pathogens through the release of cytotoxic granules and production of reactive oxygen species (ROS) (4).

The complement system consists of a cascade of sequentially activated proteins (5). Complement promotes phagocytosis and lysis of pathogens through recruitment of macrophages and neutrophils. In addition, pathogens or infected host cells can be coated by complement proteins, which are then recognised by phagocytes, resulting the destruction of the target cells (5).

In addition to directly attacking pathogens, some innate cells such as monocytes, macrophages or dendritic cells (DCs) can also act as antigen presenting cells (APCs) and induce adaptive immune responses (6). Priming of adaptive responses is facilitated by TLR triggering, which induces maturation of the APCs and renders them able to interact with components of the adaptive immune system in a productive manner (1).

\subsubsection{The Adaptive Immune System}

Adaptive immune responses can be sub-divided into humoral and cell-mediated responses. The main players in humoral immunity are B cells (7). B cells express cellsurface immunoglobulins (or B cell receptors) that recognise three-dimensional structures of antigens on pathogens or pathogen components. This antigen recognition triggers the activation of B cells, which then differentiate into antibody producing plasma cells or memory B cells (7). Antibodies specifically bind to antigens, resulting in the formation of antigen-antibody complexes that can be recognised and eliminated by cells of the innate immune system (7).

Cellular immunity is mediated by $\mathrm{T}$ cells, which express antigen specific $\mathrm{T}$ cell receptors (TCRs) and combat mostly intracellular pathogens (8). Circulating T cells can be activated by APCs in lymphoid organs and differentiate into effector T cells. Based on their effector function, $\mathrm{T}$ cells can be subdivided into cytotoxic $\mathrm{T}$ lymphocytes (CTL, also known as CD8 $\mathrm{T}$ cells) and $\mathrm{T}$ helper cells ( $\mathrm{T}_{\mathrm{H}}$ cells or CD4 T cells). CD8 T cells can 
eliminate intracellular pathogens by inducing apoptosis of infected host cells through granzymes or Fas-FasL interaction (8). CD4 T cells produce cytokines that can further enhance CTL responses or promote humoral immunity (9).

CD8 $\mathrm{T}$ cells and CD4 $\mathrm{T}$ cells have common progenitors that migrate from the bone marrow to the thymus during their development (10). Differentiation of pre-T cells into mature $\mathrm{T}$ cells in the thymus involves the rearrangement of TCR subunits, which determines the antigen specificity of the $\mathrm{T}$ cells. Once the rearrangement is complete, a functional TCR is expressed on the cell surface, and the T cells can undergo selection and maturation to CD4 or CD8 T cells, before they egress from the thymus and enter the circulation (10).

\subsection{Antigen Presentation to T Cells}

The majority of circulating $\mathrm{T}$ cells are naïve cells that have not encountered antigen (11). In order to differentiate into effector $\mathrm{T}$ cells, they require to be activated by APCs. Immature APCs are recruited to sites of infection by chemokines such as monocyte chemoattractant protein-1 (MCP-1) or chemokine (C-C motif) ligand 20 (CCL20) that are produced by resident cells in response to the infection (12). Recruited APCs acquire antigen at the infection site and migrate to secondary lymphoid organs, such as lymph nodes or the spleen, where they present antigen to $\mathrm{T}$ cells (11). Concurrently with their migration, the APCs undergo phenotypic maturation, a process that is initiated by the activation of TLR signalling pathways by microbial compounds at the site of infection (1).

APCs possess two major mechanisms of antigen presentation (6). Antigens derived from intracellular bacteria or viruses are presented on major histocompatibility complex (MHC) class I molecules, whereas antigens derived from pathogens that reside in the extracellular space or within vesicular compartments are presented on $\mathrm{MHC}$ class II molecules (13). This dichotomy ensures that the immune system can mount appropriate responses to pathogens replicating in different intracellular or extracellular compartments.

The MHC class I (MHCI) antigen presentation pathway acquires antigenic peptides within the APC (6). This pathway involves the degradation of pathogen-derived proteins by cytosolic proteasome complexes and the subsequent transport of peptides into the endoplasmic reticulum (ER), where they are loaded onto newly synthesised MHCI 
molecules (6). Peptide-loaded MHCI molecules are transported to the cell surface, where they can be recognised by TCRs on CD8 T cells. In presence of additional APC-derived co-stimulatory signals, TCR triggering results in the proliferation of the CD8 T cells and their differentiation into cytotoxic T cells (8).

The majority of pathogenic bacteria replicate in the extracellular space of their host. Antigens derived from these bacteria are not accessible to the MHCI pathway, and the immune system requires alternative means of presenting this type of antigens to $\mathrm{T}$ cells. APCs sample their environment for extracellular antigens, process and present these antigens on MHCII molecules (13). Peptide-MHCII complexes (pMHCII) are recognised by specific TCRs on CD4 T cells. In presence of appropriate co-stimulation, recognition of antigen results in the activation of CD4 $\mathrm{T}$ cells, which then differentiate into functional $\mathrm{T}_{\mathrm{H}}$ cells (11).

\subsection{Activation of CD4 T Cells}

Each naïve T cell bears about 30,000 identical TCRs on its surface (11). The core of each one of these TCRs consists of two polypeptide chains with highly variable regions termed TCR- $\alpha$ and TCR- $\beta$ (14). The TCR- $\alpha \beta$ heterodimer is associated with invariant accessory molecules, such as the CD3 complex and the TCR- $\zeta$ chain. Both the TCR- $\alpha$ and the TCR- $\beta$ chains have an N-terminal variable (V) region, which form a single antigen binding site upon dimerisation (15). In addition, both chains have an extracellular constant $(\mathrm{C})$ region for association with $\mathrm{CD} 3$, a hinge region that is responsible for dimerisation, a hydrophobic transmembrane domain and a short cytoplasmic tail (14).

TCRs on CD4 T cells respond to short contiguous peptides that are displayed on MHCII molecules on APCs in secondary lymphoid organs (11). For an efficient interaction with the APC to occur, the TCR is required to bind to both MHCII itself and the presented peptide. Upon recognition of a pMHCII complex, cytoplasmic domains of CD3 and the TCR- $\zeta$ chain become phosphorylated (16). This phosphorylation initiates signalling cascades that lead to the activation of transcription factors and altered gene expression, resulting in the overall activation of the T cell. CD4 is a co-receptor that is expressed on cells that recognise pMHCII complexes (CD4 T cells). Both the TCR and CD4 are required to interact with pMHCII for efficient activation of the T cell (11). 
In addition, a co-stimulatory signal is required for optimal activation of naïve $\mathrm{T}$ cells (11). This co-stimulatory signal must come from the same APC that is presenting antigen, and is delivered by either B7.1 (CD80) or B7.2 (CD86) (11). These two structurally related glycoproteins are exclusively expressed on professional APCs, and interact with CD28 on the surface of T cells. Hence, signals through the TCR itself, through CD4 and through CD28 are required to act in concert for optimal activation of CD4 T cells (11).

TCR signalling is not an all-or-nothing process, and can rather deliver signals of varying quality. Signalling through TCRs can trigger the production of different cytokines and the acquisition of distinct effector functions by the $\mathrm{T}$ cell, depending on the type, strength and frequency of the signal as well as the nature of co-stimulation (17). These variables also depend on the environment in which $\mathrm{T}$ cell stimulation occurs; for instance the presence of pro- or anti-inflammatory cytokines or the type of co-stimulatory molecules expressed by the APCs (17).

Activation of $\mathrm{T}$ cells is followed by the process of clonal expansion, during which $\mathrm{T}$ cells proliferate and gradually acquire properties that distinguish them as effector $\mathrm{T}$ cells $\left(\mathrm{T}_{\mathrm{H}}\right.$ cells in case of CD4 $\mathrm{T}$ cells). Once differentiated into functional $\mathrm{T}_{\mathrm{H}}$ cells, they can exert effector function through recognition of cognate pMHCII without further need for co-stimulation (11).

\subsection{CD4 T Cell Differentiation}

During clonal expansion, the pathway of differentiation of CD4 T cells is determined by the local cytokine environment, the type of co-stimulation and the nature of the pMHCII ligand (9). Hence, innate cells that present antigen, provide co-stimulation and produce cytokines play an important role in $\mathrm{T}$ cell differentiation into $\mathrm{T}_{\mathrm{H}}$ cell subsets.

Initially two different effector phenotypes of CD4 T cells were proposed (18). These were distinguished on the basis of their cytokine production patterns, where $T_{H} 1$ cells produce interferon- $\gamma(\mathrm{IFN}-\gamma)$ and promote inflammation and cell-mediated immunity; and $\mathrm{T}_{\mathrm{H}} 2$ cells produce interleukin-4 (IL-4), and are responsible for orchestrating the clearance of helminths and other extracellular pathogens by enhancing humoral immunity (17). Recently, a third subset of CD4 T cells has been discovered. This subset has been termed $\mathrm{T}_{\mathrm{H}} 17$ based on its prominent IL-17 production, and mediates 
the clearance of certain classes of pathogens that cannot be effectively dealt with by $T_{H} 1$ or $\mathrm{T}_{\mathrm{H}} 2$ cells. (19).

Cytokines are the most important factors that drive the differentiation of naïve CD4 T cells into effector cells. Macrophages, monocytes and DCs secrete IL-12 in response to TLR stimulation, and IL-12 is the major cytokine that promotes $\mathrm{T}_{\mathrm{H}} 1$ cell differentiation $(9,20) . \mathrm{T}_{\mathrm{H}} 1$ cells can activate macrophages through the production of IFN- $\gamma$, which in turn destroy microbes through ROS production or phagocytosis (3). In addition, IFN- $\gamma$ promotes the activation of CD8 $\mathrm{T}$ cells into active cytotoxic $\mathrm{T}$ cells that eliminate intracellular pathogens (8). $\mathrm{T}_{\mathrm{H}} 1$ responses are accompanied by severe inflammation, and often result in tissue damage, which stems from the toxic side effects of cytokines and other inflammatory mediators released during the immune attack on the pathogen (17).

Development of $\mathrm{T}_{\mathrm{H}} 2$ cells is commonly considered to be regulated by IL-4 (20). $\mathrm{T}_{\mathrm{H}} 2$ cells are also characterised by a pronounced production of IL-4, and additional $\mathrm{T}_{\mathrm{H}} 2$ cytokines are IL-5, IL-10 and IL-13 (20). These cytokines are critically involved in initiating and regulating immunity against extracellular pathogens, by stimulation of $\mathrm{B}$ cells to produce neutralising antibodies (9). $\mathrm{T}_{\mathrm{H}} 2$ cytokines also have anti-inflammatory functions, such as blocking IFN- $\gamma$ induced macrophage activation (17). Hence, activation of $\mathrm{T}_{\mathrm{H}} 2$ cells results in the containment of acute and chronic inflammation and limits tissue damage.

IL-6 and transforming growth factor- $\beta$ (TGF- $\beta$ ) are the major cytokines that induce differentiation of $\mathrm{T}_{\mathrm{H}} 17$ cells $(21)$. $\mathrm{T}_{\mathrm{H}} 17$ mediated immunity is considered to serve as a means to clear pathogens that cannot be effectively challenged by the other $T_{H}$ cell subsets (19). A pathogenic role of $\mathrm{T}_{\mathrm{H}} 17$ cells and associated cytokines is also implicated in autoimmune diseases in both experimental animals and humans (21).

\subsection{Tolerance}

An important feature of the immune system is its ability to distinguish between self and non-self, so that immune responses are mounted only against foreign antigens (10). The term tolerance refers to the multiplicity of mechanisms that the immune system uses to eliminate or contain cells that are potentially self-reactive (22). Central tolerance refers to the events that delete potential self-reactive lymphocytes during their 
development in the bone marrow and the thymus. Peripheral tolerance involves the elimination or suppression of mature self-reactive lymphocytes in secondary lymphoid organs or peripheral tissue (22).

\subsubsection{Central Tolerance}

Central $\mathrm{T}$ cell tolerance involves the elimination of potentially self-reactive $\mathrm{T}$ cells and takes place in the thymus (22). Developing $\mathrm{T}$ cells (thymocytes) encounter endogenous pMHC complexes, and the affinity of their TCR to these complexes determines the fate of the thymocyte. Thymocytes that fail to recognise pMHC complexes do not mature and die within the thymus (10). By this way it is guaranteed that thymocytes that cannot recognise self-MHC molecules do not mature, as they would have no function in the periphery.

Thymocytes that express TCRs with low affinity to self-peptide-MHC complexes undergo positive selection in the thymic cortex; they mature and egress to the periphery (22). Positive selection of cells that recognise self-MHC molecules also enriches the thymocyte pool for cells that are potentially self-reactive, i.e. in addition to self-MHC also recognise self-peptides. However, thymocytes with high affinity to self-pMHC complexes are deleted through induction of apoptosis in the thymic medulla (10). By this mechanism of negative selection, the immune system prevents these cells from reaching the periphery where they could become activated by APCs presenting self-peptides and cause damage to host tissue.

Efficient negative selection is ensured by the promiscuous expression of peripheral antigens by epithelial cells in the thymic medulla (22). Many antigens that are otherwise restricted to certain tissues are also expressed in the thymus. This ensures that $\mathrm{T}$ cells specific for these tissue-specific antigens do not mature. However, not all tissue specific antigens are expressed in the thymus. Hence, although central tolerance mechanisms are highly effective, they cannot eliminate all potentially self-reactive lymphocytes (22).

\subsubsection{Peripheral Tolerance}

The fact that few people develop autoimmune disorders in spite of incomplete deletion of autoreactive cells by central tolerance mechanisms indicates that additional mechanisms of tolerance exist. Autoreactive cells that evade central tolerance are controlled by mechanisms collectively known as peripheral tolerance. Peripheral 
tolerance mechanisms include anergy, ignorance and suppression of autoreactive cells (23).

T cells that have low affinity to self-pMHCII complexes escape deletion in the thymus. However, these cells do not become activated when they encounter antigen. Instead, a state of antigen specific unresponsiveness, called anergy, is induced (23). This mechanism likely plays an important role in the development of tolerance to autoantigens, because many ubiquitous self-antigens are continuously presented to $\mathrm{T}$ cells (23). However, $\mathrm{T}$ cell anergy can be overcome when the $\mathrm{T}$ cells encounter antigen presented by APCs that express high levels of co-stimulatory factors as a result of infection, resulting in activation of the $\mathrm{T}$ cell and self-directed immune responses (23).

An additional mechanism of peripheral tolerance is the presence of physical barriers between $\mathrm{T}$ cells and cognate antigen that can maintain a state of ignorance to self-antigens (24). For example, tissue-specific antigens that are only expressed in the central nervous system (CNS) are shielded from lymphocytes by the blood-brain barrier (BBB), which prevents entry of immune cells into the CNS (24). However, if the physical barrier is disrupted and becomes permeable to autoreactive cells, these self-antigens can become targets of autoimmune responses (25).

Specialised T cells with the ability to suppress immune responses can regulate the activity of autoreactive cells. Principal among these suppressive cells are a population of $\mathrm{CD}^{+} \mathrm{CD}^{+} 5^{+}$regulatory $\mathrm{T}$ cells (T-regs) (26). T-regs can be identified in mice by the expression of the transcription factor Foxp3, a key regulator of T-reg development, maintenance and function (26).

T-regs may suppress autoreactive cells through secretion of inhibitory cytokines such as IL-10 and TGF- $\beta$, secretion of pro-apoptotic factors such as granzymes, impairment of $\mathrm{T}$ cell stimulation by modulation of DCs; or consummation of IL-2, a survival factor of activated T cells, through high level expression of CD25 (IL-2 receptor $\alpha$ chain) (27). The importance of T-regs in maintaining tolerance is clearly demonstrated by the development of severe autoimmunity by both mice and humans that lack functional Foxp3 (26).

Multiple central and peripheral tolerance mechanisms are required to act in concert to prevent immune responses to self-antigens without diminishing the ability of the immune system to mount efficient responses against pathogens. Failure of one or 
several of these tolerance mechanisms can result in the initiation of self-directed immune responses and the development of autoimmune disorders.

\subsection{Autoimmunity}

Autoimmune responses resemble normal immune responses to non-self antigens, with the exception that they are specific for antigens expressed by the host, or autoantigens (25). Differences between some foreign antigens and autoantigens can be subtle; hence lymphocytes that recognise pathogen-derived antigens can also be reactive to autoantigens (10).

The most common autoimmune diseases are thought to arise from specific activation of self-reactive lymphocytes by autoantigens, or by foreign antigens that closely resemble autoantigens. However, the antigens that incite autoimmunity are difficult to identify, due to the spontaneous nature of most autoimmune disorders. The study of autoantibodies and specificity of self-reactive $\mathrm{T}$ cells provides a means to identify target antigens of autoimmune responses (28).

Immune responses to pathogens generally cease after the elimination of the pathogen. However, this pattern of resolution does not apply to autoimmune disorders, due to the vast excess and continuous synthesis of autoantigens (10). Hence, autoimmune diseases tend to develop into a chronic condition, accompanied by inflammation and tissue damage (25). As a result, more autoantigens are released, and additional autoantigens may become targets of immune responses, a phenomenon termed epitope spreading (28). Hence, autoimmune diseases cannot be cured or reversed once they are established.

Two major patterns of autoimmune diseases can be distinguished. In systemic autoimmune disorders, immune responses are directed to ubiquitous autoantigens; for example in systemic lupus erythematosus, immune responses are directed against chromatin or the spliceosome complex (29). In contrast, in organ-specific autoimmune diseases, immune attack is directed against particular host organs or tissues. Examples of organ-specific autoimmunity are insulin-dependent diabetes mellitus or multiple sclerosis, in which immune responses target $\beta$ cells in pancreatic islets or the myelin sheath that isolates nerve fibres in the CNS, respectively $(25,30)$. 


\subsection{Multiple Sclerosis}

Multiple sclerosis (MS) is an inflammatory disease of the CNS white matter, characterised by infiltration of the CNS by $\mathrm{T}$ lymphocytes and macrophages, demyelination and axonal damage (31). Destruction of CNS tissue results in neurological deficits such as sensory loss, limb weakness, incontinence, cognitive defects and paralysis (32). MS has complex genetic determinants of susceptibility, and contribution of infectious agents to disease initiation or progression is also possible (33).

\subsubsection{Statistics and Epidemiology}

MS typically manifests between the ages of 20 and 40. It is more common in females than in males and estimated to affect 2.5 million people worldwide (25) and about one in 1000 people in New Zealand ${ }^{1}$. The prevalence of MS is significantly higher in populations in high latitudes such as in Europe and Australasia (33). However, MS is much less common among Maori people in New Zealand than among New Zealanders of European origin (34), indicating that the genetic background, rather than the geographic location, may be the determinant of susceptibility.

The contribution of genetic determinants to disease susceptibility is illustrated by the fact that monozygotic twins have a concordance rate of $25-30 \%$ to develop MS, compared to only 3-5\% in dizygotic twins and non-twin siblings (35). To date, HLA-DR2 is the only locus that is linked to MS with certainty. However, the inheritance pattern of MS resembles those of polygenic diseases, and other loci may also contribute to susceptibility. Genetic factors account only in part for disease susceptibility, since monozygotic twins remain $70-75 \%$ discordant (35), suggesting that both genetic determinants and environmental factors play a role in the development of MS.

The clinical course of MS is generally categorised as being either relapsingremitting (RRMS) or primary progressive (PPMS). RRMS is the disease type present in $85-90 \%$ of total MS patients, and is characterised by a series of attacks that result in varying degrees of disability from which patients recover partly or completely, usually followed by a remission period of variable duration before another attack (32). The course of disease in about $40 \%$ of RRMS patients ultimately changes to a progressive form known as secondary-progressive MS (SPMS, ref. (31)). Progressive forms of the disease lack acute attacks, but rather involve a gradual clinical decline. An additional

\footnotetext{
${ }^{1}$ The Multiple Sclerosis Society of New Zealand (MSSNZ), http://www.msnz.org.nz/
} 
form, chronic relapsing MS, is characterised by a progressive disease course from onset with occasional relapses later in disease (35).

The prognosis of MS is widely variable. On average, patients require unilateral assistance in ambulation fifteen years after diagnosis without treatment. At the same time, $10-15 \%$ of patients even require a wheelchair, whereas $20-25 \%$ of the patients are unrestricted in their ambulance (35).

It is known that both genetic and environmental factors contribute to MS susceptibility, but the disease-initiating event is unclear. Some infectious agents like Epstein-Barr virus, human herpes virus 6 or

have been suggested to be involved in the onset of MS, but evidence for a causative link between infectious agents and MS is currently lacking (35). Investigating the cause of MS is particularly challenging, because disease is artificially induced in experimental models of MS (36). Therefore, even though these models are valuable for investigating immunological mechanisms, they cannot serve as a platform for studying possible causes of MS.

\subsubsection{Murine EAE as a Model of Human MS}

Experimental autoimmune encephalomyelitis (EAE, also known as experimental allergic encephalomyelitis) is a disease model of the immune system responding to priming with CNS-restricted self-antigens, that resembles human MS (36). Initial studies were carried out by Louis Pasteur and Thomas M. Rivers in the early $20^{\text {th }}$ century on rabbits. Since then, EAE has been replicated in a range of species, including guinea pigs, mice, rats, pigs, dogs, sheep and primates (37).

Susceptibility to EAE is variable among species, strains or even commercial sources of the animals. For instance, generally mice are more resistant to EAE than rats (37). In addition, some mouse strains are resistant to EAE induction (such as BALB/c), whereas some other strains (such as C57BL/6J) are susceptible (37).

Two different types of EAE are distinguished: active EAE and passive EAE. Active EAE is induced by immunisation with homogenised CNS tissue or purified myelin components such as myelin basic protein (MBP), myelin oligodendrocyte glycoprotein (MOG) or proteolipid protein (PLP) emulsified in Complete Freund's Adjuvant (CFA) (37). CFA is an emulsion that contains heat-killed

and paraffin oil, and ensures the gradual and constant release of the encephalitogenic peptides. The addition of mycobacteria amplifies the immune response by antigen non-specific immune stimulation through PRRs, and is required for 
overcoming peripheral tolerance and the initiation of autoimmunity (37). In addition, injection of pertussis toxin is also required for the development of clinical EAE in mice (37).

Passive EAE is induced by the adoptive transfer of myelin-reactive CD4 T cells from immunised animals into naïve recipients (37). Under these conditions, EAE develops more quickly, is less variable and injection of mycobacteria into the recipients is avoided. However, the generation of myelin-reactive $\mathrm{T}$ cell clones requires timeconsuming restimulation with antigen (37).

EAE models have several genetic, immunological and histopathological features common to MS. For instance, genetic susceptibility to MS is associated with the MHC, as is susceptibility to EAE in mice $(38,39)$. The major pathological features common in both mice and humans include the destruction of the myelin sheath that isolates nerve fibres with limited damage to the fibres themselves and the presence of multiple CNS lesions (36).

Immunologically, both EAE and MS are considered to be $\mathrm{T}_{\mathrm{H}} 1$ mediated diseases (25). In addition, $\mathrm{T}_{\mathrm{H}} 17$ cells contribute substantially to disease pathogenesis, certainly in EAE and probably in MS $(19,40) . \mathrm{T}_{\mathrm{H}} 1 / \mathrm{T}_{\mathrm{H}} 17$ cells infiltrate the CNS, and recruit and/or activate additional cell types like CD8 T cells, macrophages or neutrophils. These recruited cells are the main causes of CNS tissue damage in both EAE and MS (38).

EAE models have proven very valuable because they are rapid models that exhibit many of the features of human MS (38). Therefore EAE models can be used to investigate whether the mechanism of action of a particular drug is likely to translate into a treatment for the human disease. The value of EAE is demonstrated by several drugs that were developed in EAE models and have either been approved or have shown promise in clinical trials for MS treatment, such as glatiramer acetate (GA) and mitoxantrone $(41,42)$.

An obvious limitation of the murine EAE model is the lack of diversity in mouse strains as opposed to outbred human populations, which makes it difficult to estimate the proportion of responder patients in clinical trials based on data from mouse studies (38). MS is a disease with great heterogeneity in clinical manifestation and extent of disability, and this heterogeneity cannot be reproduced in EAE. In addition, MS treatment is often initiated at times of long-established disease, whereas EAE treatment is usually commenced concomitantly with disease induction or directly after the onset of disease. 
Hence the immunological and pathological prerequisites at the time of EAE treatment cannot be readily extrapolated to the situation in MS patients (38).

EAE is the first identified and best-characterised animal model of human autoimmune disease (36). It resembles human MS in several immunological and pathophysiological features. Despite its limitations, EAE offers great overall value for the study of demyelinating autoimmune disease, and has a strong track record in the development of drugs that are currently in clinical use for MS treatment (38).

\subsubsection{Pathogenesis of MS and EAE}

The immunopathogenesis of MS is characterised by an autoimmune attack against components of the oligodendrocyte myelin sheath that isolates axons in the CNS (25). Inflammation is initiated by the activation of myelin-specific $\mathrm{T}$ cells in the periphery, which subsequently infiltrate the CNS through the permeated BBB and recruit other types of inflammatory cells. These recruited cells are responsible for the destruction of the oligodendrocyte myelin sheath and damage to the axons (25).

Under normal conditions, $\mathrm{T}$ cells specific for autoantigens are anergised or suppressed by peripheral tolerance mechanisms (10). In MS, tolerance is overcome and myelin-specific CD4 T cells become activated in the periphery by an as yet unknown mechanism (28). In EAE, self-reactive $\mathrm{T}$ cells are activated by APCs presenting artificially introduced myelin antigens. These APCs express high levels of co-stimulatory molecules that enable them to induce immunity to the myelin antigens, rather than tolerance (31). This mature APC phenotype is a consequence of TLR stimulation through bacterial components that are contained in CFA (36).

Concurrently with their activation by APCs, CD4 T cells differentiate into proinflammatory $\mathrm{T}_{\mathrm{H}}$ cells (38). IFN- $\gamma$ producing $\mathrm{T}_{\mathrm{H}} 1$ cells were previously believed to be the main lymphocyte subset involved in the initiation of MS and EAE. However, recent studies have demonstrated that $\mathrm{T}_{\mathrm{H}} 17$ cells likely play a substantial role in the development of EAE as well (43). In addition, IL-17 expression is elevated in the CSF of MS patients (43), suggesting that $\mathrm{T}_{\mathrm{H}} 17$ cells may also be involved in the pathogenesis of the human disease.

Mice that lack IL-17 are partially resistant to EAE, whereas IFN- $\gamma$ deficient mice show exacerbated EAE $(44,45)$. However, pure populations of IL-17 producing $\mathrm{T}_{\mathrm{H}} 17$ cells cannot induce passive EAE, whereas IFN- $\gamma$ secreting $\mathrm{T}_{\mathrm{H}} 1$ populations can (46). In 
EAE, $T_{H} 1$ and $T_{H} 17$ cells are differentially stimulated by different encephalitogenic peptides, and adoptively transferred CD4 T cells induce different forms of EAE, depending on the $\mathrm{T}_{\mathrm{H}} 1: \mathrm{T}_{\mathrm{H}} 17$ ratio in the transferred population (47). Hence, regulation of CNS autoimmunity by $\mathrm{T}_{\mathrm{H}} 1$ and $\mathrm{T}_{\mathrm{H}} 17$ cells is complex, and both subsets probably contribute to the initiation and progression of EAE.

Activated myelin-specific CD4 $\mathrm{T}$ cells (probably both $\mathrm{T}_{\mathrm{H}} 1$ and $\mathrm{T}_{\mathrm{H}} 17$ ) are able to penetrate the BBB and enter the CNS. Increased expression of cell adhesion molecules on endothelial cells adjacent to CNS lesions is well documented in both MS and EAE (31). Activated CD4 T cells can bind to these adhesion molecules and extravasate through the endothelium. Activated CD4 T cells also produce matrix metalloproteases (MMPs) that promote the breakdown of the BBB by degrading type IV collagen, the main component of the basement lamina $(31,48)$. Blockage of selected endothelial adhesion molecules (such as activated leukocyte cell adhesion molecule (ALCAM) or very late antigen-4 (VLA-4)), as well as MMPs (such as MMP9) can reduce severity and delay the onset of EAE $(25,49)$, suggesting that permeation of the BBB to allow entry of CD4 T cells into the CNS is a key event in the pathogenesis.

Inflammatory CD4 $\mathrm{T}$ cells infiltrate the CNS and recognise myelin antigens that are presented by CNS resident APCs such as microglia (50). Recognition of antigen reactivates the CD4 $\mathrm{T}$ cells and stimulates them to produce inflammatory mediators such as cytokines, chemokines or MMPs (51). While increased levels of MMPs further promote BBB breakdown, chemokines and cytokines recruit and activate additional inflammatory cell types, such as CD8 T cells, B cells, neutrophils or macrophages (25, 31). These recruited cells cause damage to CNS tissue through a number of different mechanisms (25).

CD4 T cells are crucial for the initiation of both MS and EAE (25), but their role in the effector phase of the diseases is probably limited to the recruitment of other inflammatory cell types. CD8 $\mathrm{T}$ cells are the main $\mathrm{T}$ cell population that are found in active MS lesions, and adoptive transfer of activated myelin-specific CD8 T cells can induce passive EAE (25). CD8 T cells contribute to destruction of the myelin sheath and axonal damage directly through their cytotoxic granules or by receptor-mediated induction of apoptosis, for instance through Fas-FasL interaction (25).

In addition to CD8 T cells, disease severity of both MS and EAE correlates with CNS-infiltrating macrophages (52). Macrophages can amplify the local inflammatory 
response by presenting antigens to $\mathrm{T}$ cells (25). Moreover, activated macrophages directly cause tissue damage through production of MMPs, ROS or nitric oxide (NO). Free radicals like ROS and NO cause pronounced cytotoxicity during CNS inflammation in both MS and EAE (25).

Macrophages and CD8 T cells are important contributors to tissue damage in both EAE and MS. In addition, autoantibodies that are produced by activated B cells and neutrophil-derived cytotoxic intermediates such as NO (25) also cause demyelination in EAE and may contribute to tissue damage in MS (50). Hence, a number of different cell types cause demyelination and axonal damage through different mechanisms, but disease initiation requires the activation of myelin specific CD4 T cells (25).

In early stages of MS, autoreactive CD4 T cells are likely to be restricted in specificity to one or few self-antigens. In later stages, $\mathrm{T}$ cells specific for an increasing number of myelin antigens become activated, and this epitope spreading is also observed in EAE (28). Even though EAE is usually induced by immunisation with a single myelin protein epitope, $\mathrm{T}$ cells reactive to other epitopes of the same protein become activated, followed by $\mathrm{T}$ cell activation to other myelin proteins (28).

Autoimmune responses can only be triggered when mechanisms of tolerance fail. Antigen specific anergy or regulatory $\mathrm{T}$ cells normally inhibit self-specific $\mathrm{T}$ cells in circulation. In mice, Foxp3 $3^{+}$T-regs are essential for the prevention of spontaneous autoimmune responses (53), and adoptive transfer of $\mathrm{CD} 25^{+}$cells can inhibit active EAE. In contrast, depletion of $\mathrm{CD} 25^{+}$cells results in exacerbation of disease (54). There is no numerical deficiency in T-regs in MS patients compared to healthy controls, but T-regs from MS patients show impaired ability to suppress proliferation of responder $\mathrm{T}$ cells (53). Therefore, impaired T-reg function probably contributes to the development of MS.

In summary, immunological events that promote pathogenesis of EAE and MS include the activation of myelin-specific $\mathrm{T}_{\mathrm{H}} 1$ and $\mathrm{T}_{\mathrm{H}} 17$ cells in the periphery, which is rendered possible by deficient peripheral tolerance mechanisms. The myelin-specific cells then transmigrate through the permeated BBB and become reactivated in the CNS. Upon reactivation, the $T_{H} 1 / T_{H} 17$ cells produce inflammatory cytokines and chemotactic factors that potentiate local inflammation and recruit additional inflammatory cell types. Among these recruited cells, mainly CD8 T cells and macrophages are responsible for the damage to CNS tissue. 


\subsubsection{Current MS Treatment}

The cornerstone of current management of RRMS is the acute treatment of relapses with corticosteroids (CS) and long-term treatment with disease-modifying agents such as $\beta$-interferons (IFN- $\beta$ ) or glatiramer acetate (GA). Second-line treatment in nonresponders include more aggressive agents including natalizumab and mitoxantrone, and trials with combinations of disease-modifying drugs are ongoing (55).

CS are a class of steroid hormones that have strong anti-inflammatory properties. High dose short-term oral or parenteral CS therapy is in common use for the treatment of acute exacerbations of inflammatory diseases (56). This therapy provides symptomatic relief, improves motor function and accelerates recovery from relapses in RRMS patients (56). CS are considered to act by suppressing lymphocyte proliferation and by inhibiting MMPs, thus reducing traffic of activated myelin-reactive lymphocytes into the CNS (56). However, CS treatment is associated with a number of adverse effects, including depression, insomnia, hypertension, diabetes, gastritis and oedema (56).

Natalizumab (Tysabri ${ }^{\circledR}$, Biogen Idec/Elan), a humanised monoclonal antibody directed against the cell adhesion molecule $\alpha_{4} \beta_{1}$ integrin, is the most recent drug to be approved for treatment of RRMS (57). By blocking of $\alpha_{4} \beta_{1}$ integrin, natalizumab prevents CNS infiltration by immune cells (57). Treatment with natalizumab reduces relapse frequency and impedes the overall disability progression more efficiently than any other RRMS treatment approved to date (55). However, blockage of $\alpha_{4} \beta_{1}$ integrin also impairs the capability of the immune system to effectively combat infections (58). Natalizumab treatment can render patients susceptible to opportunistic infections, such as progressive multifocal leukoencephalopathy, a viral infection that almost exclusively occurs in severely immunocompromised patients (58). As a result, the use of natalizumab is largely restricted to patients that do not respond to other disease-modifying treatments and is only prescribed after vigorous risk-benefit assessment (58).

Mitoxantrone (Novantrone ${ }^{\circledR}$, EMD Serono) is approved for treatment of RRMS, SPMS and relapsing progressive MS (41). It exert cytotoxicity on proliferating cells by introducing double strand breaks in the DNA (59). Mitoxantrone has also non-cytotoxic immunosuppressive effects, and inhibits proliferation of T cells, B cells and macrophages (59). However, severe adverse effects such as cardiac dysfunction and therapy-related acute leukaemia have been associated with mitoxantrone treatment (41). Hence 
mitoxantrone is considered to be a treatment option for patients who experience insufficient response to IFN- $\beta$ or GA.

IFN- $\beta$ are approved for RRMS treatment, and are used as a first-line therapeutic. At present, two types of recombinant human IFN- $\beta$ are in use: IFN- $\beta_{1 \text { a }}$ (Avonex ${ }^{\circledR}$, Rebif $^{\circledR}$ ) and IFN- $\beta_{1 b}\left(\right.$ Betaferon $\left.^{\circledR}\right)$ (60). The therapeutic effect is considered to be predominantly due to the induction of a $\mathrm{T}_{\mathrm{H}} 2$ favouring cytokine shift through modulation of cytokine production by APCs. In addition, IFN- $\beta$ treatment reduces transmigration of autoreactive lymphocytes through the BBB (60). The result of the treatment is a reduction of relapse frequency and disease progression of about 35\% (61). IFN- $\beta$ are generally well tolerated, but a major drawback of the use of $\beta$-IFN in MS treatment is the development of neutralising antibodies to IFN- $\beta$, which render one third of the patients unresponsive to treatment one year after its initiation (61).

\subsubsection{Glatiramer Acetate}

Glatiramer acetate (GA, Copaxone ${ }^{\circledR}$, Teva Pharmaceuticals) is a random copolymer of L-alanine, L-glutamic acid, L-lysine and L-tyrosine in a molar ratio of 4.2:3.4:1.4:1.0, respectively, with a degree of polymerisation of 45-100 (62). These amino acids are the main constituents of MBP, a major encephalitogen implicated in MS pathogenesis and used to induce EAE in mice (42).

The initial purpose in synthesising GA was to develop a synthetic polypeptide that could mimic MBP to be used for the induction of EAE. Instead, GA was found to suppress EAE under certain conditions (42). EAE suppression by GA has been replicated in a number of species, including mice, rats and primates (42). Following these preclinical studies, GA successfully went through clinical trials, and was approved by the Food and Drug Administration for the treatment of RRMS in 1996 (42).

In RRMS treatment, GA is subcutaneously administered daily at a dose of $20 \mathrm{mg}$. This treatment significantly reduces the frequency of relapses in RRMS patients and delays the overall progression of the disease (63). Sustained inhibition of disease progress and improvement of neurological function by long-term GA treatment has also been reported (64).

GA is generally well tolerated and has an excellent risk-benefit profile in RRMS patients. Apart from single case reports on skin necrosis and hepatitis, only mild and transient adverse effects are known (65-67). These include mild injection site reactions 
including inflammation and capillary congestion, as well as immediate post-injection reactions such as chest tightness, palpitations, shortness of breath or chest pain (65).

\section{Mechanism of Action}

The mechanism of action of GA is not fully understood. Most of the knowledge on its mechanism of action has been obtained in EAE studies using mice or rats. In spite of some differences between treatment of EAE and human MS, the mechanism of disease alleviation by GA has significant overlap in the rodent and human disease (62).

The induction of anti-inflammatory $\mathrm{T}$ cells is the best researched feature of GA treatment, and a shift from $\mathrm{T}_{\mathrm{H}} 1$ to $\mathrm{T}_{\mathrm{H}} 2$ is well established in both mice and humans (6870). GA-induced $\mathrm{T}_{\mathrm{H}} 2$ cells are able to cross the BBB and exert immunomodulation and suppress ongoing inflammatory processes in the CNS by secreting anti-inflammatory cytokines (71). Consistent with this, disease inhibition correlates with increased production of anti-inflammatory $\mathrm{T}_{\mathrm{H}} 2$ cytokines such as IL-4, IL-10, IL-5 and TGF- $\beta$ (72).

The generation of an anti-inflammatory milieu in the CNS antigen nonspecifically inhibits the recruitment and function of inflammatory cells that mediate damage to CNS tissue, an effect termed bystander suppression (73). This feature of the drug not only allows suppression of EAE induced by a number of encephalitogens, but is also of particular benefit in MS treatment, where epitope spreading causes immune reactions directed against multiple antigens (33).

CNS-infiltrating $\mathrm{T}_{\mathrm{H}} 2$ cells can also secrete soluble factors that directly prevent tissue damage. GA-reactive $\mathrm{T}$ cells in the brain of treated mice secrete brain-derived neurotrophic factor (BDNF), a neurotrophin family growth factor that can protect axons from pathological insult (74). GA-specific T cells isolated from treated MS patients also produce BDNF after restimulation

(75). In addition, CNS-infiltrating $\mathrm{T}_{\mathrm{H}} 2$ cells may also have the potential to induce the production of BDNF and other neuroprotective factors by CNS resident cells (76).

In addition to the initiation of $\mathrm{T}_{\mathrm{H}} 2$ responses, GA treatment also results in the antigen-specific expansion of Foxp3 ${ }^{+}$T-regs in both mice (77) and humans (78). T-regs are able to inhibit myelin-directed $\mathrm{T}$ cell responses, and adoptive transfer of GA-induced T-regs efficiently suppresses EAE (77). Hence Foxp3 ${ }^{+}$T-regs appear to be significantly involved in suppression of EAE, and probably of human MS as well $(78,79)$.

GA treatment initiates both anti-inflammatory and regulatory $\mathrm{T}$ cell responses. Interestingly, GA-T $\mathrm{T}_{\mathrm{H}} 2$ cells and T-regs are mutually exclusive populations in mice (80). 
Hence GA may suppress EAE by two different non-overlapping $\mathrm{T}$ cell mediated mechanisms. However, T cell mediated mechanisms are not sufficient to explain the full extent of protection in EAE. Selective ablation of $\mathrm{T}_{\mathrm{H}} 2$ or regulatory $\mathrm{T}$ cell function does not completely abrogate protection from EAE in GA treated mice $(81,82)$. T cell independent mechanisms such increased lymphocyte apoptosis have been proposed (83), but data in support of these hypotheses are currently lacking. Therefore additional, as yet unidentified mechanisms are likely to contribute to GA therapy.

GA does not affect $\mathrm{T}$ cells directly (84), therefore the induction of protective $\mathrm{T}$ cell responses likely occurs indirectly through effects of GA on cells that present antigen to $\mathrm{T}$ cells (80). Co-culture of CD4 T cells with monocytes or DCs that have been previously exposed to GA results in the preferential secretion of $\mathrm{T}_{\mathrm{H}} 2$ cytokines such as IL-4 and IL-5 $(84,85)$. In contrast, production of IFN- $\gamma$ by the T cells is reduced. These findings are consistent with the idea that modulation of APCs by GA is responsible for the increased presence of $\mathrm{T}_{\mathrm{H}} 2$ cells in treated mice and MS patients (86). In further support of an important role for APCs in GA treatment, monocytes isolated from GA treated mice produce increased amounts of IL-10 and TGF- $\beta$, and reduced TNF- $\alpha$ and IL-12 in comparison to untreated mice (80). These monocytes are also able to present antigen and induce differentiation of $\mathrm{T}_{\mathrm{H}} 2$ cells and T-regs (80).

The mechanism by which GA exerts immunomodulation on APCs is currently not known. GA can directly bind to MHCII molecules on the surface of APCs regardless of the MCHII haplotype and without internalisation or processing (88). It is not known whether GA can induce T cells after direct binding to MHCII. However, GA

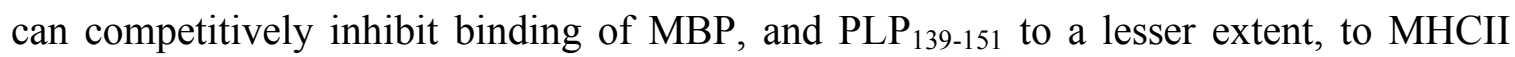
molecules $(89,90)$, and inhibit MBP specific $\mathrm{T}$ cell responses by acting as a TCR antagonist (91). This mechanism of effector $\mathrm{T}$ cell inhibition may contribute to GA's efficacy in suppressing EAE. However, the importance of TCR antagonism in the treatment of the human disease remains in dispute (62).

In addition to modulating APCs to induce protective $\mathrm{T}$ cell responses, it has also been suggested that GA contributes actively to neural repair and neurogenesis (76). Elevated proliferation of oligodendrocyte progenitor cells has been observed in brains of GA-treated EAE mice. These progenitors migrated towards regions of tissue damage, and might differentiate into mature oligodendrocytes and replace damaged myelin (76). Consistent with this, mice treated with GA after the onset of clinical EAE often exhibit 
full recovery of neurological function (76). However, it is not clear whether repair of damaged CNS tissue occurs in GA treated MS patients.

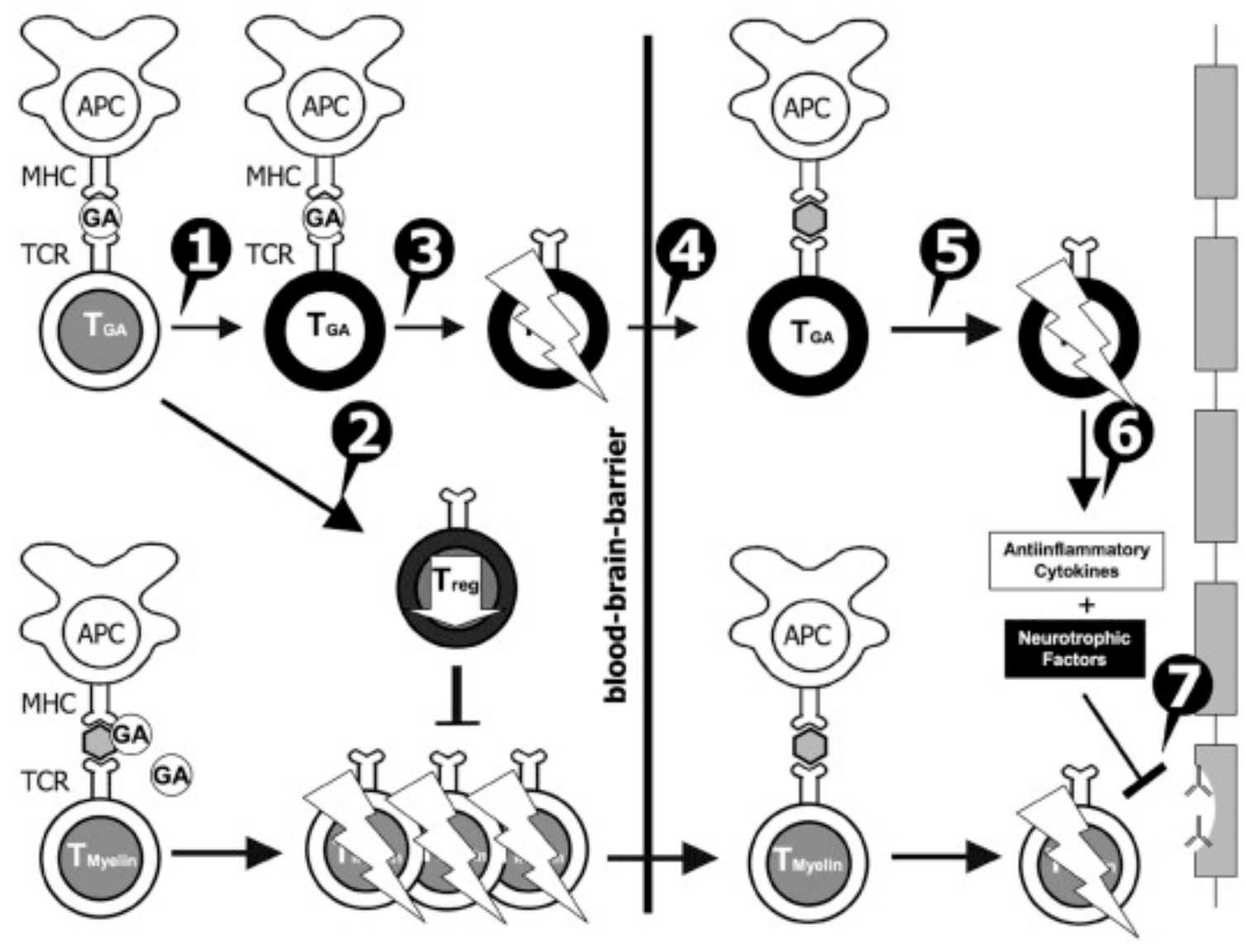

Figure 1.1: Mechanism of Action of Glatiramer Acetate (GA) in EAE Suppression.

1: GA primarily acts on APCs in the periphery, rendering them able to induce antigenspecific $\mathrm{T}_{\mathrm{H}} 2$ cells. 2: In addition, GA-induced APCs also induce T-regs that are able to suppress pathogenic $\mathrm{T}$ cells in the periphery. 3,4: Constant activation by peripheral APCs renders the $T_{H} 2$ cells able to infiltrate the CNS. 5,6: After crossing the BBB, GA-specific $\mathrm{T}$ cells become reactivated and secrete anti-inflammatory cytokines that alleviate ongoing CNS inflammation. 7: In addition, $\mathrm{T}_{\mathrm{H}} 2$ cells also secrete neurotrophic factors or stimulate local cells to do so, which contributes to protection from axonal damage and supports remyelination. Adopted from ref. (63).

In summary, the current data on the mechanism of action of GA suggests that GA primarily acts on APCs in peripheral tissue, rendering the APCs able to induce antiinflammatory and regulatory $\mathrm{T}$ cell responses (figure 1.1). These T-regs can then suppress pathogenic $\mathrm{T}$ cell responses in the periphery. In addition, GA-induced $\mathrm{T}_{\mathrm{H}} 2$ cells infiltrate the CNS after their activation and can exhibit bystander suppression 
producing anti-inflammatory cytokines that dampen inflammation and neuroprotective factors that prevent damage to CNS tissue.

\subsection{Objectives}

GA is one of the most efficient therapies currently available for the treatment of MS. Currently available data suggests that the primary mode of action involves antigen specific expansion of anti-inflammatory and regulatory $\mathrm{T}$ cells (62), which likely results from a GA-induced phenotype shift in APCs (86).

GA is only effective when administered daily at high doses, over long time periods (63). Hence strategies aimed at improving the efficacy of GA, thereby reducing the therapeutic dose, are desirable. The first objective of this study was to improve GA therapy in a murine EAE model, by conjugation to high affinity targeting molecules that specifically bind to cell surface components on APCs. This targeting strategy was hypothesised to substantially increase uptake of GA by the APCs and presentation of GA-derived peptides to CD4 $\mathrm{T}$ cells. Hence the targeting strategy exploits the antigen specific mechanisms of GA to achieve a significant reduction of the therapeutic dose in EAE. Drug targeting is currently a field of extensive research, and targeting GA to its target cells has the potential for clinical application in MS treatment.

The primary target of GA are considered to be innate cells with the ability of antigen presentation, but experimental evidence of direct effects of GA on such cells

is currently unavailable. Furthermore, the specific target of GA within the multiplicity of cell types that can present antigen is unknown.

The second objective of this study was to identify the target cell population of GA. Additional aims were to characterise the target cells and assess their ability to exert direct $\mathrm{T}$ cell immunosuppression. Antigen non-specific suppression of autoreactive cells may contribute to the efficacy of GA in EAE and MS, and gaining a complete picture of the mechanism of action of GA may lead to the development of improved immunomodulatory therapies. 


\section{CHAPTER 2:}

MATERIALS \& METHODS 


\subsection{Mice}

\subsubsection{Maintenance and Ethic Approvals}

All mice were bred and maintained at the Biomedical Research Unit of the Malaghan Institute of Medical Research, based at Victoria University of Wellington, New Zealand. Experiments were approved by the Victoria University Animal Ethics Committee and performed under the guidelines of the Victoria University, under the license numbers 2007R8M (Investigating the cause and inhibitory schemes of experimental autoimmune encephalomyelitis, a murine model for human multiple sclerosis) or 2006R19 (The use of mouse tissues for multiple research projects).

\subsubsection{Mouse Strains}

C57BL/6J $\left(\mathrm{H}-2^{\mathrm{b}}\right)$ mice were originally purchased from the Jackson Laboratory (Bar Harbour, ME, USA).

2D2 mice have a transgenic TCR specific for the $\mathrm{H}-2^{\mathrm{b}}$ binding $\mathrm{MOG}_{35-55}$ peptide. The mouse strain was generated by microinjection of cDNA encoding a $\mathrm{MOG}_{35-55}$ specific TCR, which expresses V $\alpha 3.2$ and V $\beta 11$ TCR segments, into fertilised oocytes of C57BL/6J mice (92). 2D2 mice were obtained from Prof. Vijay K. Kuchroo (Harvard Medical School, Boston, MA, USA).

OT-II mice have a transgenic TCR specific for the $\mathrm{H}-2^{\mathrm{b}}$ binding $\mathrm{OVA}_{323-339}$ peptide. The OT-II mice are on C57BL/6J background and were originally obtained from Dr. William Heath (Walter and Eliza Hall Institute for Medical Research, Melbourne, VIC, Australia).

OT-I mice have a transgenic TCR specific for the $\mathrm{K}^{\mathrm{b}}$ binding $\mathrm{OVA}_{257-264}$ peptide. The strain was generated by microinjection of cDNA encoding the specific TCR into C57BL/6J blastocysts. OT-I mice are on C57BL/6J background and were obtained from Prof. Frank Carbone (University of Melbourne, Melbourne, VIC, Australia).

B6.SJL-ptprc ${ }^{\text {a }}$ mice were developed by intercrossing C57BL/6J mice with SJL/J mice. This mouse strain is genetically similar to $\mathrm{C} 57 \mathrm{BL} / 6 \mathrm{~J}$ strain except that it carries the $\operatorname{ptprc}^{\text {a }}$ (protein tyrosine phosphatase receptor type c locus) allele (CD45.1) from the SJL/J strain. B6.SJL-ptprc ${ }^{\mathrm{a}}$ mice were purchased from Animal Resource Centre (Canning Vale, WA, Australia). 
$\mathrm{B} \mathrm{Aa}^{0} / \mathrm{Aa}^{0}$ mice lack the expression of MHC class II molecules. The strain was generated by the targeted mutation of the Aa gene, which encodes one of the polypeptide chains of the IA molecule. $\mathrm{B} \mathrm{Aa}^{0} / \mathrm{Aa}^{0}$ mice were obtained from Dr. H. Bluethmann (Hoffmann-La Roche, Basel, Switzerland).

\subsubsection{Genotyping of TCR Transgenic Mouse Strains}

TCR transgenic animals were genotyped by determining the expression of the transgenic TCR on peripheral blood lymphocytes by flow cytometry. Mice were tail bled and blood cells were prepared by water lysis as described in section 2.6.2. Cells were then stained with antibodies specific for V $\alpha 2$ and V $\beta 5.1-5.2$ for OT-II mice, or V $\alpha 3.2$ and V $\beta 11$ for 2D2 mice. Stained cells were analysed by flow cytometry and transgenic mice were defined as those that contained both transgenic TCR $\alpha$ and TCR $\beta$ segments.

\subsection{Chemicals}

Chemicals were purchased from the following companies, as depicted in section 2.3:

BDH Chemicals, distribution by VWR, West Chester, PA, USA

Invitrogen, Carlsbad, CA, USA

Merck KGaA, Darmstadt, Germany

PAA, Pasching, Austria

Roche, Basel, Switzerland

Serva GmbH, Heidelberg, Germany

Sigma-Aldrich, St. Louis, MO, USA. 


\subsection{Buffers and Solutions}

\subsubsection{Buffers for Immunisations}

Pertussis toxin buffer

Tris $15 \mathrm{mM} \quad$ Invitrogen

Sodium chloride $\quad 0.5 \mathrm{M} \quad \mathrm{BDH}$

Triton X-100 $\quad 0.017 \%(v / v) \quad$ Serva

in MilliQ water (Q-Gard ${ }^{\circledR} 1$ Purification Pack, Millipore Corp., Billerica, MA, USA), pH adjusted to 7.4 with $\mathrm{HCl}$. Filter sterilised using a $0.22 \mu \mathrm{m}$ vacuum driven disposable bottle top filter (Steritop ${ }^{\mathrm{TM}}$, Millipore). Stored at $4{ }^{\circ} \mathrm{C}$, used sterile.

\subsubsection{Buffers and Media for Cell Preparation and Tissue Culture}

Alsever's solution

D-glucose

$114 \mathrm{mM}$

Sigma-Aldrich

Sodium citrate

$27 \mathrm{mM}$

Sigma-Aldrich

Sodium chloride

$71 \mathrm{mM}$

in MilliQ water, $\mathrm{pH}$ adjusted to 6.1 with $1 \mathrm{M}$ citric acid (BDH). Filter sterilised, stored at $4^{\circ} \mathrm{C}$ and used sterile.

\begin{tabular}{lll}
\multicolumn{2}{l}{ Complete Iscove's Modified Dulbecco's Medium (cIMDM) } \\
2-Mercaptoethanol & $55 \mu \mathrm{M}$ & Invitrogen \\
Foetal calf serum (FCS) & $5 \%(\mathrm{v} / \mathrm{v})$ & PAA \\
Non-essential amino acids & $100 \mu \mathrm{M}$ & Invitrogen \\
Penicillin & $1 \mathrm{U} / \mathrm{ml}$ & Invitrogen \\
Sodium pyruvate & $1 \mathrm{mM}$ & Invitrogen \\
Streptomycin & $1 \mu \mathrm{g} / \mathrm{ml}$ & Invitrogen
\end{tabular}

in sterile IMDM (Invitrogen). Stored at $4{ }^{\circ} \mathrm{C}$ for no longer than 14 days, used sterile. 


\section{DNase/Collagenase solution}

DNase I

$0.2 \mathrm{mg} / \mathrm{ml}$

Roche

Collagenase Type II

$1 \mathrm{mg} / \mathrm{ml}$

Invitrogen

in sterile cIMDM. Prepared fresh before each use, used sterile.

\section{Red blood cell lysis buffer}

Tris

$20 \mathrm{mM}$

Ammonium chloride

$14 \mathrm{mM}$

$\mathrm{DBH}$

in MilliQ water, $\mathrm{pH}$ adjusted to 7.4 with $\mathrm{HCl}$. Sterilised by autoclaving, stored at room temperature (RT) and used sterile.

\section{Würzburger buffer}

DNAse I

$0.02 \mathrm{mg} / \mathrm{ml}$

FCS

$1 \%(\mathrm{v} / \mathrm{v})$

EDTA

$5 \mathrm{mM}$

Invitrogen

in sterile Dulbecco's phosphate buffered saline (D-PBS, Invitrogen). Stored at $4{ }^{\circ} \mathrm{C}$, used sterile.

\subsubsection{Buffers for Cell Purification}

FlowComp ${ }^{\mathrm{TM}}$ isolation buffer

FCS

$2 \%(\mathrm{v} / \mathrm{v})$

EDTA

$1 \mathrm{mM}$

in sterile D-PBS. Stored at $4^{\circ} \mathrm{C}$, used sterile.

autoMACS ${ }^{\mathrm{TM}}$ running buffer

FCS $1 \%(\mathrm{v} / \mathrm{v})$

EDTA $2 \mathrm{mM}$

in D-PBS. Sterilised by autoclaving, FCS added after sterilisation. Stored at $4^{\circ} \mathrm{C}$ and used sterile. 
autoMACS $^{\mathrm{TM}}$ rinsing buffer

EDTA

$2 \mathrm{mM}$

in D-PBS, sterilised by autoclaving. Stored at $4^{\circ} \mathrm{C}$, used sterile.

\subsubsection{Buffers for Flow Cytometry}

\section{FACS buffer}

Sodium azide

$0.1 \%(\mathrm{w} / \mathrm{v})$

Sigma-Aldrich

FCS $1 \%(\mathrm{v} / \mathrm{v})$

EDTA

$2 \mathrm{mM}$

in D-PBS, stored at RT.

\subsubsection{Solutions for Preparation of $\mathrm{Cl}_{2} \mathrm{MDP}$ Liposomes}

\section{Phosphatidylcholine stock solution}

L- $\alpha$-phosphatidylcholine $\quad 100 \mathrm{mg} / \mathrm{ml} \quad$ Sigma-Aldrich

in chloroform (Merck). Stored at $-20{ }^{\circ} \mathrm{C}$

\section{Cholesterol stock solution}

Cholesterol $20 \mathrm{mg} / \mathrm{ml} \quad$ Sigma-Aldrich

in chloroform. Stored at $-20^{\circ} \mathrm{C}$

\section{Dichloromethylene diphosphonate $\left(\mathrm{Cl}_{2} \mathrm{MDP}\right)$ solution}

$\mathrm{Cl}_{2} \mathrm{MDP}$

$0.7 \mathrm{M}$

Sigma-Aldrich

in MilliQ water, $\mathrm{pH}$ adjusted to 7.1 with $\mathrm{NaOH}$. Stored at $-20{ }^{\circ} \mathrm{C}$ 


\subsubsection{Solutions for Immunohistochemistry}

\section{Zinc fixative}

Tris

$0.1 \mathrm{M}$

Calcium acetate

$3 \mathrm{mM}$

Sigma-Aldrich

Zinc acetate

$27 \mathrm{mM}$

Sigma-Aldrich

Zinc chloride

$37 \mathrm{mM}$

Sigma-Aldrich

in MilliQ water, $\mathrm{pH}$ adjusted to 7.4 with $\mathrm{HCl}$. Stored at RT.

\subsubsection{Solutions for SDS PAGE}

\section{Staining solution}

Coomassie $^{\circledR}$ brilliant blue G 250

$$
0.1 \%(\mathrm{w} / \mathrm{v})
$$

Serva

Glacial acetic acid

$$
10 \%(\mathrm{v} / \mathrm{v})
$$

Merck

Methanol

$45 \%(\mathrm{v} / \mathrm{v})$

Merck

in MilliQ water. Stored at room temperature (RT).

\section{Destaining solution}

Glacial acetic acid

$$
\begin{aligned}
& 7.5 \%(\mathrm{v} / \mathrm{v}) \\
& 5 \%(\mathrm{v} / \mathrm{v})
\end{aligned}
$$

Methanol

in MilliQ water. Stored at RT.

\subsubsection{Buffers for Chromatography}

\section{FPLC running buffer}

Disodium hydrogen phosphate $\quad 8.1 \mathrm{mM} \quad \mathrm{BDH}$

Sodium dihydrogen phosphate $\quad 11.9 \mathrm{mM} \quad \mathrm{BDH}$

in MilliQ water, $\mathrm{pH}$ adjusted to 7.0. Filtered through $0.22 \mu \mathrm{m}$ filter (Steritop ${ }^{\mathrm{TM}}$, Millipore), stored at RT. 


\section{FPLC elution buffer}

Glycine

$0.1 \mathrm{M}$

$\mathrm{BDH}$

in MilliQ water, $\mathrm{pH}$ adjusted to 2.7 with $\mathrm{HCl}$. Filtered through $0.22 \mu \mathrm{m}$, stored at RT.

\section{FPLC neutralisation buffer}

Tris

in MilliQ water, $\mathrm{pH}$ adjusted to 9.0 with $\mathrm{HCl}$. Filtered through $0.22 \mu \mathrm{m}$, stored at RT.

\section{CNBr sepharose coupling buffer}

Disodium hydrogen phosphate $\quad 92.5 \mathrm{mM}$

Sodium dihydrogen phosphate $\quad 7.5 \mathrm{mM}$

Sodium chloride $\quad 0.5 \mathrm{M}$

in MilliQ water, $\mathrm{pH}$ adjusted to 8.3. Filtered through $0.22 \mu \mathrm{m}$, stored at RT.

\section{CNBr sepharose blocking buffer}

Tris

$0.1 \mathrm{M}$

in MilliQ water, $\mathrm{pH}$ adjusted to 8.0 with $\mathrm{HCl}$. Filtered through $0.22 \mu \mathrm{m}$, stored at RT.

\section{CNBr Sepharose low pH wash buffer}

Glacial acetic acid

$$
0.5 \%(\mathrm{v} / \mathrm{v})
$$

Sodium acetate

$15 \mathrm{mM}$

$\mathrm{BDH}$

Sodium chloride

$0.5 \mathrm{M}$

in MilliQ water, $\mathrm{pH}$ adjusted to 4.0. Filtered through $0.22 \mu \mathrm{m}$, stored at RT.

\section{CNBr sepharose high pH wash buffer}

Tris

$0.1 \mathrm{M}$

Sodium chloride

$0.5 \mathrm{M}$

in MilliQ water, $\mathrm{pH}$ adjusted to 8.0 with $\mathrm{HCl}$. Filtered through $0.22 \mu \mathrm{m}$, stored at RT. 


\subsection{Proteins, Peptides and Copolymers}

MOG $_{35-55}$ peptide (MEVGWYRSPFSRVVHLYRNGK) was synthesised by Mimotopes (Clayton, Victoria, Australia). This $\mathrm{H}-2^{\mathrm{b}}$ binding peptide corresponds to amino acids $35-55$ of the murine MOG protein. Purity of the peptide was $>97 \%$.

OVA $_{323-339}$ (ISQAVHAAHAEINEAGR) is an $\mathrm{H}-2^{\mathrm{b}}$ binding peptide derived from the chicken OVA sequence and corresponds to amino acids 323-339. OVA $323-339$ was synthesised by Mimotopes ( $>95 \%$ pure).

Recombinant MOG protein corresponds to the N-terminal extracellular domain (amino acids 1-117) of the murine MOG protein. MOG protein was provided by Prof. John D. Fraser, University of Auckland.

Ovalbumin (OVA) protein was purchased from Sigma-Aldrich as a lyophilised powder. The protein is isolated from chicken egg, and has a purity of $>98 \%$.

Trypsinogen (from bovine pancreas, Sigma-Aldrich) is the inactive precursor of the intestinal protease trypsin. The molecular weight is $24 \mathrm{kDa}$ by calculation. It was purchased as an essentially salt-free, lyophilised powder and stored at $-20^{\circ} \mathrm{C}$, protected from moisture. After dissolving in PBS, it was stored at $4{ }^{\circ} \mathrm{C}$.

Glatiramer acetate $\left(\mathrm{GA}\right.$, Copaxone $\left.^{\circledR}\right)$ was purchased from Teva Pharmaceutical Industries Ltd. (Petach Tikva, Israel).

SMEZ2 M1 is a modified form of the streptococcal superantigen SMEZ2 that contains the mutations W75L.K182Q.D42C. As a consequence, this modified superantigen cannot bind TCR. SMEZ2 M1 and the conjugates SMEZ2 M1-MOG and SMEZ2 M1-OVA were provided by Prof. John D. Fraser (Department of Molecular Medicine and Pathology, University of Auckland, Auckland).

DM SMEZ2 is a modified form of the streptococcal superantigen SMEZ2 that contains the mutations Y18A.D42C.H202A.D204A. As a consequence, this modified superantigen cannot bind TCR or MHCII molecules. DM SMEZ2 was provided by Prof. John D. Fraser, University of Auckland. 


\subsection{Immunisations and Treatment}

\subsubsection{EAE induction}

Eight to twelve week old female C57BL/6J or 2D2 mice were immunised with $50 \mu \mathrm{g} \mathrm{MOG}_{35-55}$ emulsified in CFA (Difco Laboratories, Detroit, MI, USA) containing $500 \mu \mathrm{g}$ heat-killed

(Difco Laboratories). The components were emulsified by three homogenisation cycles of 90 seconds each in a Mini bead beater-8 (BioSpec Products, Bartlesville, OK, USA). The emulsion was transferred to $1 \mathrm{ml}$ syringes (BD Biosciences, Franklin Lakes, NJ, USA) by centrifugation. The mice were injected with $100 \mu \mathrm{l}$ of the emulsion subcutaneously in each hind limb flank. One day after EAE induction, mice received intraperitoneal injections of $250 \mathrm{ng}$ pertussis toxin (Sapphire Bioscience, Redfern, NSW, Australia) in $200 \mu 1$ pertussis toxin buffer (section 2.3.1).

The mice were monitored daily throughout 40 days after EAE induction. EAE scores were assigned as follows: 1, loss of tail tonicity; 2, limp tail; 3: hind limb weakness; 4: hind limb paralysis; 5: hind and fore limb paralysis, moribund state. Mice were scored and weighed daily. Mice that reached EAE score 5 or lost $>20 \%$ of their total body weights within five days were euthanised.

\subsubsection{Treatment}

Co-immunisation. Eight to twelve week old female C57BL/6J or 2D2 mice were subcutaneously immunised in the hind limb flanks with $50 \mu \mathrm{g}_{\mathrm{MOG}_{35-55}}$ and $500 \mu \mathrm{g}$ GA emulsified in CFA containing $500 \mu \mathrm{g}$ heat-killed - Mice received intraperitoneal injections of $250 \mathrm{ng}$ pertussis toxin one day after co-immunisation.

C57BL/6J mice were co-immunised with $50 \mu \mathrm{g} \mathrm{MOG}_{35-55}$ and either $5 \mu \mathrm{g}$ SMEZ2 M1-GA, $20 \mu \mathrm{g}$ M5/114-GA or $20 \mu \mathrm{g}$ M1/70-GA subcutaneously in the hind limb flanks. Mice received intraperitoneal injections of $250 \mathrm{ng}$ pertussis toxin one day after coimmunisation.

Treatment in the neck. Eight to twelve week old female C57BL/6J mice received subcutaneous injections of $500 \mu \mathrm{g}$ GA in $100 \mu \mathrm{l}$ PBS in the scruff of the neck on seven consecutive days before EAE induction.

Eight to twelve week old female C57BL6/J mice were subcutaneously treated with $500 \mu \mathrm{g}$ GA emulsified in $100 \mu \mathrm{l}$ CFA containing $500 \mu \mathrm{g}$ heat-killed in the scruff of the neck on the day of EAE induction. 
Intravenous treatment. Eight to twelve week old female C57BL/6J mice received intravenous injections of $500 \mu \mathrm{g}$ GA in $100 \mu \mathrm{l}$ PBS on the day of EAE induction.

\subsubsection{Immunisations to Prime Cells for Proliferation Assays or to Study Lymphoid Tissue Infiltration}

Eight to twelve week old C57BL/6J or B6.SJL-ptprc ${ }^{\text {a }}$ mice were subcutaneously immunised in the hind limb flanks with $500 \mu \mathrm{g}$ GA or $500 \mu \mathrm{g}$ GA-FITC emulsified in CFA containing $500 \mu \mathrm{g}$ heat-killed , or with CFA only. Eight hours to ten days after immunisation, cells were recovered from spleens, blood or draining (inguinal) lymph nodes.

\subsection{Tissue Harvest and Processing}

\subsubsection{Preparation of Cells from Spleens and Lymph Nodes}

Mice were sacrificed by carbon dioxide asphyxiation followed by cervical dislocation. Mice were pinned on a dissection tray and the fur was soaked with $70 \%$ ethanol. Dissection tools were stored in $70 \%$ ethanol to maintain sterility. The peritoneal cavity was opened and inguinal, para-aortic or mesenteric lymph nodes or spleens were removed as required. The spleens and lymph nodes were placed in cIMDM (section 2.3.2) on ice immediately after removal.

For the study of total cells populating the lymphoid tissue, 1-2 $\mathrm{ml}$ of DNase/collagenase (section 2.3.2) solution was injected into the spleens, and the flowthrough was collected in 24 well plates (BD Biosciences). The spleens were then placed in the collected solution and incubated at $37^{\circ} \mathrm{C}$ for 30 minutes. Lymph nodes were placed directly in $1 \mathrm{ml} \mathrm{DNase/collagenase} \mathrm{in} \mathrm{24-well} \mathrm{plates} \mathrm{and} \mathrm{incubated} \mathrm{as} \mathrm{above.}$ DNase/collagenase digestion was omitted for the isolation of lymphocytes from spleens and lymph nodes.

For preparation of single cell suspensions, spleens and lymph nodes were disrupted with the back of the plunger of $1 \mathrm{ml}$ syringes (BD Biosciences) and flushed with cIMDM (or with Würzburger buffer (section 2.3.2) after DNase/collagenase digestion) through $100 \mu \mathrm{m}$ nylon cell strainers (BD Biosciences) into $50 \mathrm{ml}$ tubes (BD Biosciences). The cells were centrifuged for three minutes at $2000 \mathrm{x}$ g. Lymph node cells were washed once, resuspended in cIMDM and placed on ice until further use. Spleen 
cells were resuspended in $1 \mathrm{ml}$ cIMDM, and $5 \mathrm{ml}$ of red blood cell lysis buffer (section 2.3.2) per spleen was added. The tube was gently inverted several times, and centrifuged as above to pellet white blood cells. The cells were washed once and resuspended in cIMDM, filtered through a $100 \mu \mathrm{m}$ cell strainer and placed on ice until further use.

For counting lymphocytes, $10 \mu \mathrm{l}$ of the cell suspension was diluted to $10 \mathrm{ml}$ with ISOTON $^{\circledR}$ II Diluent (Beckman Coulter, Fullerton, CA, USA) and cells in the size range of $4 \mu \mathrm{m}-8 \mu \mathrm{m}$ were counted with a $\mathrm{Z} 2^{\mathrm{TM}}$ Coulter Counter ${ }^{\circledR}$ (Beckman Coulter).

\subsubsection{Preparation of Cells from Blood}

Blood was collected from mice either by tail-bleeding or cardiac puncture. For tail-bleeding, mice were placed under a heat source for several minutes before tail bleeding. The mice were then placed in a restraining tube. The distal $0.5-1 \mathrm{~mm}$ of the tail was removed with a sharp pair of sterile scissors. The tail was gently rubbed from the base to the tip, and 4-5 drops of blood were collected in $1.7 \mathrm{ml}$ tubes containing $200 \mu \mathrm{l}$ of Alsever's solution (section 2.3.2). The tubes were placed on ice until further use.

For collecting blood by cardiac puncture, mice were sacrificed by carbon dioxide asphyxiation. Blood was collected with a $1 \mathrm{ml}$ syringe and a $24 \mathrm{G}$ needle. The mouse was laid on the back and needle was inserted horizontally from the left side of the thorax. About $1 \mathrm{cc}$ of air was withdrawn after the insertion to create a vacuum by pulling the plunger. The needle was then directed to the ventricle until blood appeared in the needle. The vacuum was maintained by further gently pulling back on the plunger. The collected blood $(500-800 \mu \mathrm{l})$ was immediately transferred to $1.7 \mathrm{ml}$ tubes containing $700 \mu \mathrm{l}$ Alsever's solution. The tubes were placed on ice until further use.

For preparation of total white blood cells for flow cytometry, red blood cells were lysed with MilliQ water (water lysis). Blood cells were resuspended in $500 \mu$ MilliQ water. An equal volume of $1.8 \% \mathrm{NaCl}$ was added after 20 seconds. The cells were then centrifuged for one minute at $10000 \mathrm{rpm}$ (IEC Micromax, Thermo Scientific, Waltham, MA, USA), and the water lysis repeated as required. The cells were then resuspended in FACS buffer (section 2.3.4) and placed on ice until further use.

For isolation of peripheral blood mononuclear cells (PBMCs) for tissue culture, blood cells were resuspended in $1 \mathrm{ml}$ sterile D-PBS. Blood from individual mice was pooled in $15 \mathrm{ml}$ tubes (BD Biosciences), and 2-4 $\mathrm{ml}$ of Lympholyte ${ }^{\circledR}-\mathrm{M}$ cell separation media (Cedarlane Laboratories Ltd., Burlington, NC, USA) was added through sterile pasteur pipettes (Sigma-Aldrich). The cells were then centrifuged for 20 minutes at 
2400 x g. Mononuclear cells were collected from the interface and transferred to a fresh tube. The cells were washed once, resuspended in cIMDM and placed on ice until further use.

For counting white blood cells, $10 \mu \mathrm{l}$ of cell suspension was mixed with $10 \mu \mathrm{l}$ 0.4\% Trypan Blue Stain (Invitrogen). The mixture was placed on a Bright Line doubleruling haemocytometer set (Boeco, Hamburg, Germany) and the living cells that excluded the dye were counted under 100x magnification with an Olympus CK40 inverted microscope (Olympus, Central Valley, PA, USA).

\subsubsection{CFSE Labelling}

Cell suspensions were prepared from blood or lymphoid tissue (sections 2.6.1 and 2.6.2). Red blood cells were not lysed during splenocyte isolation. The cells were resuspended in Hank's Balanced Salt Solution (HBSS, Invitrogen) containing $2 \mathrm{mM}$ EDTA at $20 \times 10^{7}$ cells $/ \mathrm{ml}$. Then, $20 \mu 1$ of carboxyfluorescein succinimidyl ester (CFSE, $200 \mu \mathrm{M}$ stock in DMSO, Invitrogen) was added to the cells. The cells were incubated for seven minutes at RT, with occasional inversions. At the end of incubation, $30 \mathrm{ml}$ of cIMDM was added and the cells centrifuged at $2000 \mathrm{x} g$ for 5 minutes. The cells were then washed twice and resuspended in HBSS (without EDTA) or cIMDM for adoptive transfers or tissue culture, respectively.

\subsection{Cell Purification}

\subsubsection{Magnetic Sorting of CD11b ${ }^{\text {hi }}$ Cells}

$\mathrm{CD}_{11} \mathrm{~b}^{\text {hi }}$ cells were isolated with the autoMACS ${ }^{\mathrm{TM}}$ cell separation system (Miltenyi Biotec, Bergisch Gladbach, Germany). PBMCs were isolated from the blood as described in section 2.6.2. The cells were resuspended in $2 \mathrm{ml}$ autoMACS running buffer (section 2.3.3) and filtered through $30 \mu \mathrm{m}$ pre-separation filters (Miltenyi). To deplete dead cells, a positive selection was performed using an autoMACS separator (program "possel", Miltenyi). The negative fraction was collected, washed once and resuspended in $200 \mu 1$ running buffer. A PE-conjugated antibody specific for CD11b (clone M1/70, Pharmingen) was added $(1 \mu \mathrm{l})$, and the cells were incubated for 15 minutes on ice. The cells were then washed twice with $50 \mathrm{ml}$ running buffer and resuspended in $200 \mu \mathrm{l}$. Subsequently, $20 \mu 1$ of anti-PE beads (Miltenyi) were added, and the cells were incubated 
for 30 minutes at $4^{\circ} \mathrm{C}$ under rotation. The cells were washed once, resuspended in $2 \mathrm{ml}$ running buffer, and separated using the autoMACS separator as above. The positive fraction contained the $\mathrm{CD} 11 \mathrm{~b}^{\mathrm{hi}}$ enriched cells $\left(>80 \% \mathrm{CD} 11 \mathrm{~b}^{\mathrm{hi}}\right)$. The cells were then washed once and resuspended HBSS or cIMDM for adoptive transfer or tissue culture, respectively.

\subsubsection{Magnetic Sorting of $\mathrm{CD}^{+}$Cells}

$\mathrm{CD}^{+}$cells were isolated with the Dynabeads ${ }^{\circledR}$ FlowComp ${ }^{\mathrm{TM}}$ Mouse CD4 kit (Invitrogen). Briefly, splenocyte or lymph node cell suspensions were resuspended in $2 \mathrm{ml}$ isolation buffer (section 2.3.3) per $5 \times 10^{8}$ total cells in $5 \mathrm{ml}$ polystyrene tubes (BD Biosciences). Then, $25 \mu 1$ of FlowComp Mouse CD4 antibody (Invitrogen) was added. The cells were incubated for 10 minutes on ice, washed once ( 3 minutes at $2000 \mathrm{x}$ g) and resuspended in $4 \mathrm{ml}$ isolation buffer. Subsequently, $300 \mu \mathrm{l}$ of FlowComp Dynabeads (Invitrogen) were added and the cells were incubated for 15 minutes at $4^{\circ} \mathrm{C}$ under rotation. The tube was placed in a DynaMag ${ }^{\mathrm{TM}}-15$ magnet (Invitrogen) for two minutes. The supernatant was removed with a pipette. The tube was removed from the magnet, the cells resuspended in isolation buffer, and the magnetic separation step repeated. The cells were then resuspended in $4 \mathrm{ml}$ FlowComp release buffer (Invitrogen) and incubated for 10 min at RT under rotation. The tube was placed in the magnet for two minutes and the supernatant containing the $\mathrm{CD}^{+}$cells $\left(>95 \% \mathrm{CD}^{+}\right)$transferred to a fresh tube. The cells were washed once and resuspended in HBSS for adoptive transfer, or in cIMDM for tissue culture.

\subsection{Adoptive Transfer}

Cell pellets from blood, spleens or lymph nodes were labelled with CFSE as described in section 2.6.3. Then either $\mathrm{CD} 4^{+}$cells from lymphoid tissue or $\mathrm{CD} 11 \mathrm{~b}{ }^{\text {hi }}$ cells from blood were enriched as described in section 2.7. The cells were resuspended in HBSS.

For proliferation assays (section 2.9.3), $2-5 \times 10^{6} \mathrm{CFSE}$ labelled CD4 ${ }^{+} \mathrm{T}$ cells from 2D2 mice were adoptively transferred into CD45 congenic B6.SJL-ptprc ${ }^{\mathrm{a}}$ mice by intravenous injection into the tail vein. 
For the study of lymph node infiltration after immunisation (section 2.5.3), $2 \times 10^{5} \mathrm{CD} 11 \mathrm{~b}^{\text {hi }}$ cells from $\mathrm{C} 57 \mathrm{BL} / 6 \mathrm{~J}$ were adoptively transferred into CD45 congenic B6.SJL-ptprc ${ }^{\text {a }}$ mice by intravenous injection into the tail vein.

\subsection{Proliferation and Suppression Assays}

\subsubsection{Antigen Specific in vitro Proliferation Assays}

Splenocytes or lymphocytes were isolated from immunised or TCR transgenic mice as described in section 2.6.1. Splenocytes from TCR transgenic mice $\left(5 \times 10^{5}\right.$ cells per well) or draining lymphocytes from immunised mice $\left(1 \times 10^{6}\right.$ cells per well) were incubated in 96-well flat bottom plates (BD Biosciences) with serial dilutions of antigen.

The cultures were incubated with atmospheric air and $5 \% \mathrm{CO}_{2}$ at $37^{\circ} \mathrm{C}$ in a HeraSafe tissue culture incubator (Thermo Scientific). After 48-72 hours of incubation, $0.25 \mu \mathrm{Ci}\left[{ }^{3} \mathrm{H}\right]$-thymidine (GE Healthcare) was added, and the cultures were incubated for eight more hours. At the end of the incubation period, either $\left[{ }^{3} \mathrm{H}\right]$-thymidine incorporation was analysed immediately, or the cultures were stored at $-20^{\circ} \mathrm{C}$ until analysis. As a positive control of proliferation, splenocytes or lymph node cells were incubated with serial dilutions of a soluble anti-CD3 antibody (clone 2c11) for 48 hours, and additional eight hours after addition of $\left[{ }^{3} \mathrm{H}\right]$-thymidine.

For analysis, cells were harvested from the 96-well plates onto printed glass fibre filter mats (PerkinElmer Lifesciences Inc., Boston, MA, USA) using a Tomtec automatic 96 well plate harvester (Tomtec, Hamden, CT, USA). Filter mats were dried in a microwave oven, placed into plastic sample bags (PerkinElmer) and scintillated with Beta Scint liquid scintillation cocktail (Wallac, Turku, Finland). The sample bags were sealed (Heat sealer, Wallac) and $\left[{ }^{3} \mathrm{H}\right]$-thymidine incorporation was analysed using a 1450 Microbeta Plus liquid scintillation counter (Wallac).

\subsubsection{In vitro Suppression Assays}

Suppression of polyclonal T cell proliferation. CD11 $\mathrm{b}^{\text {hi }}$ cells were enriched from the blood of C57BL/6J mice as described in section 2.7.1. Serial dilutions of enriched $\mathrm{CD} 11 \mathrm{~b}^{\text {hi }}$ cells were co-cultured with $5 \times 10^{4} \mathrm{C} 57 \mathrm{BL} / 6 \mathrm{~J}$ splenocytes per well in presence of $2.5 \times 10^{4}$ Dynabeads $^{\circledR}$ mouse CD3/CD28 $\mathrm{T}$ cell expander beads (Invitrogen) in a round-bottom 96-well plate (BD Biosciences). The co-cultures were incubated at $37^{\circ} \mathrm{C}$ 
for 24 hours. Then, $0.25 \mu \mathrm{Ci}\left[{ }^{3} \mathrm{H}\right]$-thymidine was added and the cultures were incubated for additional eight hours. The cultures were harvested and incorporation of $\left[{ }^{3} \mathrm{H}\right]$-thymidine was analysed as described in section 2.9.1.

Suppression of antigen specific $T$ cell proliferation. Lymphocytes were isolated from 2D2 mice and labelled with CFSE and cultured with $10 \mu \mathrm{g} / \mathrm{ml} \mathrm{MOG}_{35-55}$ in a roundbottom 96-well plate $\left(5 \times 10^{4}\right.$ cells per well). Serial dilutions of enriched CD11 bi ${ }^{\text {hi }}$ cells were added to the lymphocyte culture after 24 hours of incubation. The co-culture was incubated for additional 48-72 hours, and CFSE dilution in the responder cells was analysed by flow cytometry at the end of the incubation period.

\subsubsection{Antigen specific in vivo Proliferation Assays}

CFSE labelled $\mathrm{CD}^{+}$cells from 2D2 mice were adoptively transferred into B6.SJL-ptprc ${ }^{a}$ mice $\left(2-5 \times 10^{6}\right.$ cells per mouse). The cells were mixed with either $10 \mu \mathrm{g} / \mathrm{ml} \mathrm{MOG}_{35-55}$ or with $10 \mu \mathrm{g} / \mathrm{ml} \mathrm{MOG}_{35-55}$ and $250 \mu \mathrm{g} / \mathrm{ml}$ GA before injection, with control mice receiving cells only. CFSE dilution in the responder cells in the blood and the spleen of recipients was analysed by flow cytometry 48-96 hours after cell transfers.

In an alternative setting, CFSE labelled $\mathrm{CD}^{+}$2D2 cells were adoptively transferred to B6.SJL-ptprc ${ }^{\mathrm{a}}$ mice as above. One day after cell transfers, recipients were subcutaneously immunised with $\mathrm{MOG}_{35-55}$, or co-immunised with $\mathrm{MOG}_{35-55}$ and GA, as described in section 2.5, and control mice were immunised with CFA only. CFSE dilution in the responder cell population in the draining (inguinal) lymph nodes, spleens and blood of the recipients was analysed by flow cytometry 48-96 hours after immunisation.

\subsection{Flow Cytometry}

Single cell suspensions were prepared from various tissues at $1 \times 10^{6}-$ $2.5 \times 10^{7}$ cells $/ \mathrm{ml}$. $200 \mu \mathrm{l}$ of the cell suspensions per well were transferred to a round bottom greiner 96 well plate (BD Biosciences). The cells were centrifuged for 37 seconds at $3000 \mathrm{rpm}$ (IEC Centra GP8R, Thermo Scientific), the supernatant was flicked off and the cells resuspended by vortexing. The cells were washed twice with $200 \mu \mathrm{l}$ FACS buffer (section 2.3.4) and incubated with anti-CD16/CD32 antibody (clone 2.4G2) in 50 $\mu 1$ FACS buffer for 10 minutes at RT. The cells were washed twice with FACS buffer as above, and then incubated with a mixture of fluorophor-labelled antibodies (table 2.1) 
against cell surface molecules of interest in $50 \mu \mathrm{FACS}$ buffer for 10 minutes at RT. The cells were washed twice and incubated with fluorophor-conjugated streptavidin in $50 \mu \mathrm{l}$ FACS buffer for 10 minutes at RT. The cells were then washed twice, resuspended in $200 \mu 1$ FACS buffer and transferred to Titertube ${ }^{\circledR}$ micro tubes (Bio-Rad Laboratories Inc., Hercules, CA, USA) through filter gauze.

Table 2.1: Antibodies used for flow cytometry.

\begin{tabular}{|c|c|c|c|c|}
\hline Antigen & Clone & Source & Isotype & Fluorophor \\
\hline CD4 & GK1.5 & Pharmingen & Rat IgG2b, $\kappa$ & Alexa647 ${ }^{\mathrm{a}}, \mathrm{PE}$, PerCP \\
\hline CD8 & 2.43 & homemade & Rat IgG2b, $\kappa$ & Alexa647, PE \\
\hline CD11b & $\mathrm{M} 1 / 70$ & Pharmingen & Rat IgG2b, $\kappa$ & PE, Alexa647 a \\
\hline CD18 & $\mathrm{M} 18 / 2$ & eBioscience & Rat IgG2a, $\kappa$ & Alexa647 ${ }^{\mathrm{a}}$ \\
\hline $\mathrm{IA}^{\mathrm{b}}$ & M5/114 & Pharmingen & Rat IgG2b, $\kappa$ & PE, Alexa647 ${ }^{\mathrm{a}}$ \\
\hline CD11c & N418 & homemade & $\begin{array}{l}\text { Armenian } \\
\text { hamster IgG }\end{array}$ & Biotin \\
\hline $\mathrm{F} 4 / 80$ & BM8 & eBioscience & Rat IgG2a, $\kappa$ & Biotin, Alexa488 \\
\hline Gr-1 & RB6-8C5 & Pharmingen & Rat IgG2b, $\kappa$ & PE, Alexa647 \\
\hline CD19 & eBio1D3 & eBioscience & Rat IgG2a, $\kappa$ & Biotin, Alexa647 \\
\hline PDCA-1 & JF05-1C2. & Miltenyi & Rat IgG2b, $\kappa$ & APC \\
\hline Ly6C & AL-21 & $\mathrm{BD}$ & Rat IgM, $\kappa$ & Biotin \\
\hline Ly6G & $1 \mathrm{~A} 8$ & $\mathrm{BD}$ & Rat IgG2a, $\kappa$ & Alexa647 ${ }^{\mathrm{a}}$, Biotin \\
\hline CD80 & 16-10A1 & homemade & $\begin{array}{l}\text { Armenian } \\
\text { hamster IgG }\end{array}$ & Biotin \\
\hline CD86 & PO3.1 & eBioscience & Rat IgG2b, $\kappa$ & Alexa647 \\
\hline CD45.2 & 104 & eBioscience & Mouse IgG2a, $\kappa$ & $\mathrm{PE}$ \\
\hline CD16/CD32 & $2.4 \mathrm{G} 2$ & homemade & Rat IgG2b, $\kappa$ & Alexa647 \\
\hline CD45 & $30-\mathrm{F} 11$ & Pharmingen & Rat IgG2b, $\kappa$ & PerCP \\
\hline $\mathrm{V} \alpha 3.2$ & RR3-16 & $\mathrm{BD}$ & Rat IgG2b, $\kappa$ & FITC \\
\hline $\mathrm{V} \beta 11$ & RR3-15 & $\mathrm{BD}$ & Rat IgG2b, $\kappa$ & Alexa647 ${ }^{\mathrm{a}}$ \\
\hline
\end{tabular}

a Purified unlabelled antibodies were purchased from the indicated companies and labelled with Alexa Fluor ${ }^{\circledR} 488$ or Alexa Fluor ${ }^{\circledR} 647$ (Invitrogen) as described in section 2.13.5. 


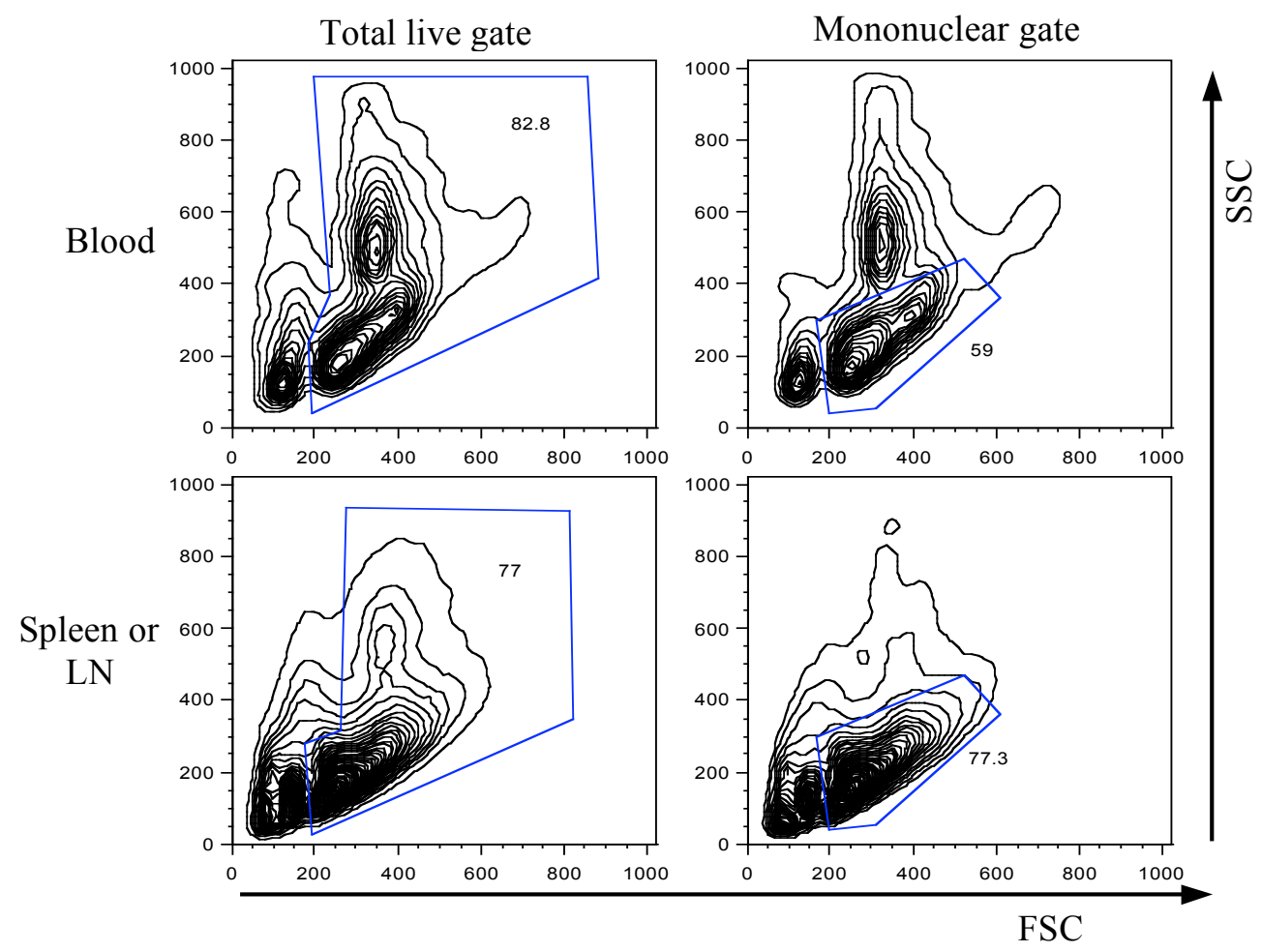

Figure 2.1: Gating on total or mononuclear cells in flow cytometry.

Contour plots acquired from unstained cell samples from blood, spleens or lymph nodes (LN) were used for gating on living cells or mononuclear cells, based on their FSC/SSC properties.

Flow cytometric analysis was performed on a FACSort (BD Biosciences). Unstained samples were used to define the population of living cells or mononuclear cells by their forward scatter (FSC) and side scatter (SSC) properties (figure 2.1). Cell samples stained with antibodies corresponding to all four channels were used to adjust the voltage, and samples stained with single antibodies were used to compensate for overflow of fluorescence into other channels. When studying a specific cell population such as $\mathrm{CD} 45.2^{+}$or $\mathrm{CD} 11 \mathrm{~b}{ }^{\text {hi }}$ cells, gates were set on these populations (figure 2.2). Data acquisition was carried out with CellQuest Pro software (BD Biosciences) and the data were analysed using the FlowJo software (Tree Star Inc., Ashland, OR, USA). 

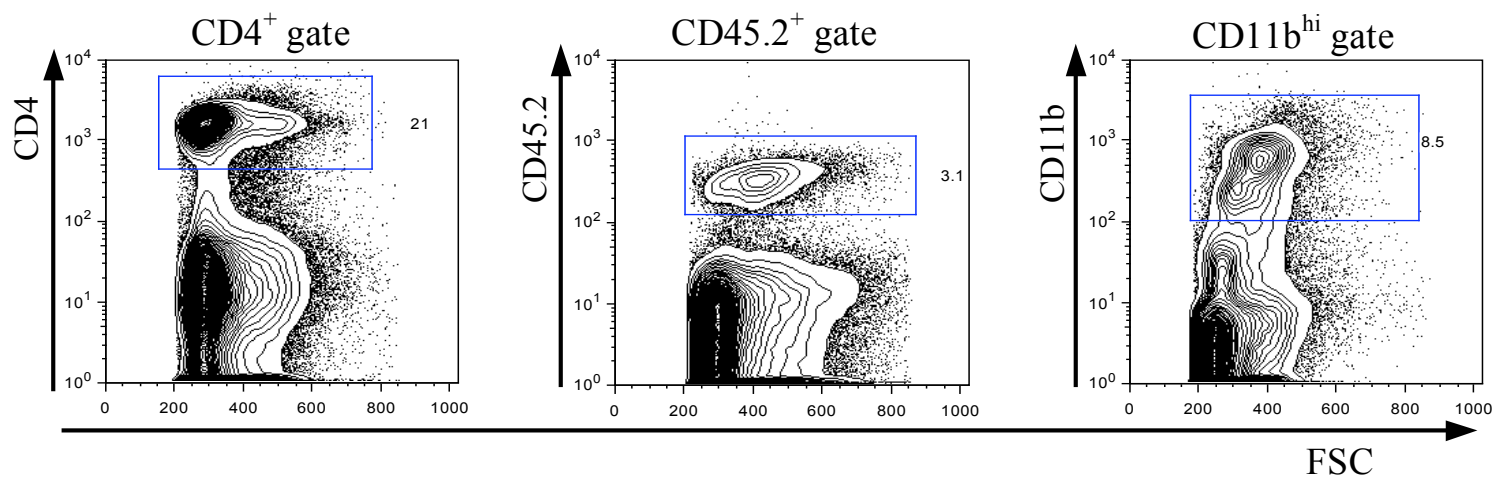

Figure 2.2: Gating based on cell surface marker expression in flow cytometry.

High-resolution contour plots with outliers were used for gating on $\mathrm{CD}^{+}, \mathrm{CD} 45.2^{+}$or $\mathrm{CD}_{11 \mathrm{~b}}{ }^{\text {hi }}$ cells. Cell samples shown are prepared from spleens of immunised C57BL/6J mice (CD4 gate), spleens of immunised B6.SJL-ptprc ${ }^{\text {a }}$ recipients of CD45.2 congenic cells (CD45.2 gate), or blood of naïve C57BL/6J mice (CD11b ${ }^{\text {hi }}$ gate). Plots are gated on mononuclear cells as shown in figure 2.1 .

\subsection{In vivo Monocyte Depletion}

For preparation of $\mathrm{Cl}_{2} \mathrm{MDP}$-liposomes, $1.1 \mathrm{ml}$ of $\mathrm{L}$ - $\alpha$-phosphatidylcholine stock solution (section 2.3.5) was added to $0.51 \mathrm{ml}$ cholesterol stock solution (section 2.3.5) in a $500 \mathrm{ml}$ round bottom flask. The solvent was removed by low vacuum rotation evaporation at 150 rpm (Rotavapor R-210, Büchi Labortechnik AG, Flawil, Switzerland) and 120 mbar (Vacuum controller V-850, Büchi) until a thin milky-white phospholipid film formed against the inside of the flask. The phospholipid film was dispersed in $10 \mathrm{ml}$ of $0.7 \mathrm{M} \mathrm{Cl}_{2} \mathrm{MDP}$ solution (section 2.3.5) by gentle rotation (120 rpm) at RT for 10 minutes. The resulting suspension was held under nitrogen gas at RT for two hours. The suspension was then gently shaken and sonicated in a waterbath at $40 \mathrm{kHz}$ (Ultrasonic cleaner FXP8M, Unisonics, Manly Vale, NSW, Australia) for three minutes. The suspension was held under nitrogen gas overnight at $4^{\circ} \mathrm{C}$.

The liposome suspension was transferred to a $50 \mathrm{ml}$ tube and centrifuged for 40 minutes at $3500 \mathrm{~g}$ and $10^{\circ} \mathrm{C}$. The aqueous phase containing the non-encapsulated $\mathrm{Cl}_{2} \mathrm{MDP}$ was removed with a $10 \mathrm{ml}$ syringe. The $\mathrm{Cl}_{2} \mathrm{MDP}$ solution was stored at $-20^{\circ} \mathrm{C}$ for further use. The liposomes were washed twice with $20 \mathrm{ml} \mathrm{D}-\mathrm{PBS}\left(3500 \mathrm{~g}\right.$ at $10^{\circ} \mathrm{C}$ for 20 minutes) and resuspended to a final volume of $5.1 \mathrm{ml}$ D-PBS. Liposomes were stored for up to one week at $4{ }^{\circ} \mathrm{C}$ before use. 
For monocyte depletion, the $\mathrm{Cl}_{2} \mathrm{MDP}$ liposomes were transferred to $1 \mathrm{ml}$ insulin syringes (BD Biosciences), and $200 \mu 1$ liposomes were intravenously injected into the tail vein of mice. Immediately before transfer to syringes and before injecting, the suspensions were gently shaken to ensure even dispersion.

\subsection{Histology}

EAE was induced in mice as described in section 2.5.1. Mice were sacrificed 40 days after EAE induction and spinal cords removed. For this, the dorsal fur was soaked with $70 \%$ ethanol and the skin was cut open from caudal to cranial. The head was removed and a cut was introduced to the spine about $5 \mathrm{~cm}$ from the cranial end with a sharp pair of sterile scissors. An $18 \mathrm{G}$ needle was inserted into the neural tube from the caudal end of the dislocated spine, and the spinal cord was removed by flushing with cold D-PBS.

Spinal cords were fixed in immunohistochemistry zinc fixative (section 2.3.6). Tissue was embedded in paraffin, sectioned at $6 \mu \mathrm{m}$ and stained with hematoxylin and eosin by the Pathology Department at the Wellington School of Medicine. Histology slides were analysed with an Olympus BX551 microscope and Olympus DP70 digital camera (Olympus, Center Valley, PA, USA).

\subsection{Biochemical Methods}

\subsubsection{Non-reducing SDS PAGE}

Non-reducing sodium dodecyl sulfate polyacrylamide gel electrophoresis (SDS PAGE) was used to evaluate the efficiency of conjugations. For this, the sample was mixed with 4x LDS sample buffer (Invitrogen) and loaded on NuPAGE ${ }^{\circledR}$ Novex Bis-Tris gels (Invitrogen). Precision Plus Protein prestained standard (Bio-Rad) was added to one

well of the gel. The gels were run using an XCell $\quad{ }^{\mathrm{TM}}$ Mini-Cell (Invitrogen) in MES SDS running buffer (Invitrogen) at constant voltage of $200 \mathrm{~V}$ from a PowerPac 3000 power supply (Bio-Rad) for 40-45 minutes. The gels were then stained with Coomassie blue (section 2.3.7) for one hour with constant shaking. The gels were destained (section 2.3.7) for one hour, and boiled for one minute in a microwave oven 
with soft tissue paper in distilled water $\left(\mathrm{dH}_{2} \mathrm{O}\right)$. Gel pictures were taken with a Molecular Imager Gel Doc XR (Bio-Rad).

\subsubsection{Conjugation of proteins to antibodies}

The conjugation system used for antibody conjugations consists of two complementary amine-reactive chemicals: C6-S-HyNic (Solulink, San Diego, CA, USA) incorporates aromatic hydrazines into proteins, whereas sulfo-S-4FB (Solulink) incorporates aromatic aldehydes. Once coupled to the respective proteins, these chemicals are only reactive to each other and form a bis-aryl-hydrazone bond upon coincubation. The chemicals were dissolved in dimethylformamide (DMF, Solulink) at a concentration of $10 \mathrm{mg} / \mathrm{ml}$ and stored as aliquots of $10 \mu 1$ at $-70^{\circ} \mathrm{C}$ until use.

For conjugation, the antibodies were first modified with C6-S-HyNic, and the proteins were modified with sulfo-S-4FB. For this, proteins and antibodies were resuspended in modification buffer (Solulink) with ZEBA desalting columns (Pierce, Rockford, IL, USA) or Vivaspin 10K MWCO PES columns (Sartorius AG, Göttingen, Germany). C6-S-HyNic or sulfo-S-4FB were then added to the antibodies or proteins, respectively, and incubated for two hours at RT. Excess chemical was removed from the solutions with ZEBA desalting columns or by three consecutive concentration-dilution cycles in Vivaspin 10K MWCO PES columns.

The modified antibodies were then mixed together with the modified proteins and incubated for one hour at RT. Conjugation efficiency was tested by non-reducing SDS PAGE. The conjugates were sterilised with $0.22 \mu \mathrm{m}$ microcentrifuge filters (Corning Inc., Corning, NY, USA) before further use.

\subsubsection{Conjugation of GA to Superantigens}

For conjugation of GA to superantigens, the heterobifunctional amine/sulfhydrylreactive crosslinking chemical SMCCplus ${ }^{\mathrm{TM}}$ (ABD Bioquest, Sunnyvale, CA, USA) was used. SMCCplus was dissolved in $50 \%(\mathrm{v} / \mathrm{v})$ of acetonitrile $/ \mathrm{dH}_{2} \mathrm{O}$ at a concentration of $50 \mathrm{mg} / \mathrm{ml}$ and aliquoted to $20 \mu \mathrm{l}$. The chemical was then dried in a Savant SpeediVac concentrator (Thermo Scientific) and the resulting dry aliquots were stored at $-20^{\circ} \mathrm{C}$ protected from moisture.

Prior to use for conjugation reactions the amount of remaining reactive chemical was determined. For this, a $1 \mathrm{mg}$ aliquot of SMCCplus was dissolved in $100 \mu \mathrm{l}$ of $50 \% \mathrm{DMF} / \mathrm{dH}_{2} \mathrm{O}$, and $10 \mu \mathrm{l}$ of the dissolved SMCCplus was transferred to $500 \mu \mathrm{l}$ of 
$\mathrm{dH}_{2} \mathrm{O}$, which was subsequently split in two tubes of $250 \mu 1$ each. To one of the tubes, $10 \mu 1$ of $2 \mathrm{~N} \mathrm{NaOH}$ was added to hydrolyse SMCCplus. The $\mathrm{OD}_{280}$ of both samples was measured immediately. The amount of reactive chemical that will conjugate to the proteins was calculated from the difference in absorbance of the hydrolysed $(\mathrm{H})$ and the unhydrolysed (UH) sample using the following formula: $\alpha=1-\left(\mathrm{A}_{280 \mathrm{UH}} / \mathrm{A}_{280 \mathrm{H}}\right)$.

GA was resuspended in D-PBS with ZEBA desalting columns and modified with $((100 * \mathrm{n}(\mathrm{GA} / \mu$ moles $)) / \alpha) \mu 1$ SMCCplus (2-fold molar excess over GA) for two hours at RT. GA was diluted to $0.75 \mathrm{mg} / \mathrm{ml}$ during modification to avoid precipitation in the DMF-containing solution. Excess chemical was removed by three consecutive concentration-dilution cycles with D-PBS in Vivaspin 3K MWCO columns (Sartorius).

Superantigens were reduced with $1 \mathrm{mM}$ Tris(2-carboxyethyl)phosphine hydrochloride (TCEP-HCl, Pierce) for 30 minutes at RT. The reducing agent was then removed with ZEBA desalting columns, and reduced superantigen was used immediately for conjugation reactions. Reduced superantigen was co-incubated with a 10-fold molar excess of modified GA over night at $4{ }^{\circ} \mathrm{C}$. Conjugation efficiency was tested by nonreducing SDS PAGE.

\subsubsection{Fast protein liquid chromatography (FPLC)}

Preparation of Gel Filtration Columns. Pre-swollen Sephacryl ${ }^{\circledR} \quad 300-\mathrm{HR}$ suspension (Sigma-Aldrich) was resuspended by gentle shaking. For one $8 \mathrm{ml}$ column, $15 \mathrm{ml}$ of the suspension was transferred to a $50 \mathrm{ml}$ tube (BD Biosciences). The suspension was centrifuged for two minutes at $2000 \mathrm{~g}$ and resuspended in MilliQ water to a total volume of $10 \mathrm{ml}$. An empty glass Econo-column (Bio-Rad) was connected to a BioLogic LP low-pressure chromatography system (Bio-Rad). The column outlet was disconnected from the chromatography tubing and sealed, and the column bed was prewet with 2-3 $\mathrm{ml}$ of FPLC running buffer (section 2.3.8). A $2 \mathrm{ml}$ glass pipette was inserted into the column, with the tip of the pipette immersing into the buffer in the bottom of the column, but not touching the column bed. The Sephacryl suspension was then slowly poured into the column alongside the wall of the glass pipette. The pipette was slowly withdrawn from the column and the gel resin let settle for 10 minutes by gravity. Then, an Econo-column flow adaptor (Bio-Rad) was inserted into the column and sealed 0.5-1 $\mathrm{cm}$ above the gel resin. The column was then packed at a flow rate of $6.5 \mathrm{ml} / \mathrm{min}$ with 
FPLC running buffer (section 2.3.8) for one hour. If not proceeding directly to a separation run, the column was filled with $20 \%$ ethanol, sealed and stored at $4{ }^{\circ} \mathrm{C}$.

Gel filtration chromatography. Gel filtration columns were connected to the tubing of the FPLC system, and the flow adaptor was inserted into the column and sealed $0.5-1 \mathrm{~cm}$ above the gel resin. The gel filtration column was washed thoroughly with running buffer at $1 \mathrm{ml} / \mathrm{min}$, until the conductivity became constant. The sample was resuspended in $200 \mu \mathrm{l}$ FPLC running buffer using Vivaspin $10 \mathrm{kDa}$ MWCO columns. The sample was then injected through the sample injection port, and the separation was performed at $1 \mathrm{ml} / \mathrm{min}$. Fractions of $1.5 \mathrm{ml}$ were collected in $1.7 \mathrm{ml}$ Eppendorf tubes using a BioFrac fraction collector (Bio-Rad) and concentrated to 20-30 $\mu 1$ using Vivaspin 10K MWCO columns. Efficiency of separation was tested by non-reducing SDS PAGE.

Affinity chromatography. Affinity chromatography was performed using two different systems. First, a pre-packed HiTrap Protein G column (GE Healthcare, Piscataway, NJ, USA) was used, which binds specifically to the immunoglobulin (Ig) Fc region. The samples were resuspended in FPLC running buffer. The chromatography column was washed with running buffer, and the samples were applied through the sample injection port. The column was thoroughly washed with running buffer until no protein was detected in the effluent. The antibodies bound to the column were then eluted with elution buffer (section 2.3.8). Fractions of $1.3 \mathrm{ml}$ were collected in $1.7 \mathrm{ml}$ Eppendorf tubes containing $200 \mu \mathrm{l}$ neutralisation buffer (section 2.3.8). The fractions were concentrated to 50-100 $\mu$ with Vivaspin $10 \mathrm{kDa}$ MWCO columns and analysed by nonreducing SDS PAGE.

Second, affinity columns were used based on the monoclonal antibody MAR18.5, which is specific for rat Ig $\kappa$ chains. CNBr-activated Sepharose ${ }^{\mathrm{TM}}$ beads (Amersham, Uppsala, Sweden) were used for the preparation a MAR18.5 affinity column. For this, MAR18.5 was coupled to the Sepharose beads following the manufacturer's instructions. Briefly, the beads were swelled in $1 \mathrm{mM} \mathrm{HCl}$, resuspended in coupling buffer (section 2.3.8) and incubated with MAR18.5 for two hours. The beads were then blocked to avoid non-specific binding, washed several times in alternating $\mathrm{pH}$ (section 2.3.8) and resuspended in FPLC running buffer. The column was packed as described for the gel filtration columns. Affinity chromatography was performed with the MAR18/5 columns as described for the HiTrap Protein G columns. 


\subsubsection{Fluorophor Labelling of Antibodies and Proteins}

Labelling with fluorescein isothiocyanate (FITC). FITC (Sigma-Aldrich) was dissolved in DMSO (Sigma-Aldrich) at $20 \mathrm{mg} / \mathrm{ml}$, and stored at $-20^{\circ} \mathrm{C}$ in $10 \mu 1$ aliquots. Proteins were resuspended in $50 \mathrm{mM}$ borate buffer at $1 \mathrm{mg} / \mathrm{ml}$ using ZEBA desalting columns. While vortexing, $5 \mu \mathrm{g}$ of FITC per $100 \mu \mathrm{g}$ of protein was added, followed by incubation at RT for 45 minutes. Remaining reactive dye was sequestered by the addition of 0.1 Vol. $1 \mathrm{M}$ Tris- $\mathrm{Cl}(\mathrm{pH} 8.5)$. The labelled protein was subsequently resuspended in D-PBS using Vivaspin size exclusion columns with the appropriate molecular cut-off (Sartorius). The proteins were washed with D-PBS until no coloration of the filtrate was visually detectable.

Labelling with Alexa Fluor succinimidyl esters or Sulfo-NHS biotin. Alexa Fluor 488, 555 or 647 (Invitrogen) or Sulfo-NHS biotin (Invitrogen) were dissolved in DMSO at $10 \mathrm{mg} / \mathrm{ml}$ and stored at $-20^{\circ} \mathrm{C}$ in $10 \mu \mathrm{l}$ aliquots. Proteins or antibodies were resuspended in freshly made $0.1 \mathrm{M} \mathrm{NaHCO}_{3}$ using ZEBA desalting columns at $2 \mathrm{mg} / \mathrm{ml}$ (antibodies) or $0.5 \mathrm{mg} / \mathrm{ml}$ (proteins). $10 \mu \mathrm{g}$ of fluorescent dye or biotin was added per $100 \mu \mathrm{g}$ of antibody, or $50 \mu \mathrm{g}$ of protein, respectively. The samples were incubated at RT for 8 minutes and the labelling reaction stopped by the addition of $0.1 \mathrm{Vol}$ of $1 \mathrm{M}$ Tris-Cl ( $\mathrm{pH} \mathrm{8.5).} \mathrm{The} \mathrm{labelled} \mathrm{proteins} \mathrm{were} \mathrm{then} \mathrm{resuspended} \mathrm{in} \mathrm{D-PBS} \mathrm{using} \mathrm{Vivaspin} \mathrm{columns}$ with the appropriate cut-off and washed until the filtrate was transparent. Labelling of antibodies was validated by flow cytometry.

\subsection{Statistical Analysis}

Statistical analysis was performed on cell proliferation data or EAE scores using a two-tailed t-test when testing statistical significance between two data sets. When comparing multiple sets of EAE scores, repeated measures ANOVA was used followed by Tukey's multiple comparison test. Statistical analysis was carried out with the software GraphPad Prism (GraphPad Software Inc., San Diego, CA, USA). 


\section{CHAPTER 3:}

\section{TARGETING GLATIRAMER}

ACETATE TO ANTIGEN

\section{PRESENTING CELLS IN EAE}

TREATMENT 


\subsection{Introduction}

Therapeutic efficacy of GA treatment in MS and EAE is commonly associated with the expansion of anti-inflammatory $\mathrm{T}_{\mathrm{H}} 2$ cells and T-regs $(62,77,80)$. However, $\mathrm{T}$ cell mediated protection does not result from direct effects of GA on T cells (84). Rather, the observed $\mathrm{T}$ cell expansion is a consequence of the action of GA on innate cells that can present antigen to T cells (86). Although APCs are considered to be the primary

target of GA, it is not known whether GA has the same effect on all APCs or preferentially acts through a specific subset. Nevertheless, a characteristic feature of the mode of action of GA appears to be the MHCII restricted presentation of GA peptides to CD4 $\mathrm{T}$ cells, which then become activated and can inhibit or counteract pathogenic events in EAE and MS $(62,63,86)$.

\subsubsection{The MHCII Antigen Presentation Pathway}

Several classes of pathogens replicate inside membrane-surrounded compartments of the cell, and proteins derived from such pathogens are inaccessible to the cytosolic proteasome complex. Instead, these proteins are degraded within acidified endosomal compartments (93). The enzymes involved in degradation of these proteins are proteases that are activated at low $\mathrm{pH}$, the best-characterised ones of which are the cathepsin family of proteases (93). Extracellular pathogens or pathogen components, as well as phagocytosed fragments of apoptotic or necrotic host cells are also degraded in endosomal compartments after their uptake by the APC. Short peptides emerge as degradation products and are subsequently loaded onto MHCII molecules for presentation to CD4 T cells (93).

MHCII molecules consist of two transmembrane subunits, the $\alpha$ - and $\beta$-chains, both of which are co-translationally translocated into the ER (13). In the ER, the $\alpha$ - and $\beta$-chains assemble to functional MHCII molecules containing one peptide-binding groove (13). A short polypeptide called the invariant chain (Ii) prevents premature loading of MHCII molecules with ER-resident peptides by binding to and blocking the peptidebinding groove (13).

An additional function of the $\mathrm{Ii}$ is the direction of MHCII to late endosomes where peptide loading occurs (figure 3.1). MHCII molecules are first transported from the ER to the Golgi complex, and pass through its cisternae to the trans-Golgi network (TGN) (94). An endosomal sorting signal that is contained within the sequence of the Ii 
mediates transport of the MHCII molecules from the TGN to late endosomes (94). Late endosomal compartments are the sites where the MHCII pathway intersects with the endocytic pathway. These compartments are therefore referred to as MIIC (MHCII containing compartment) (93).

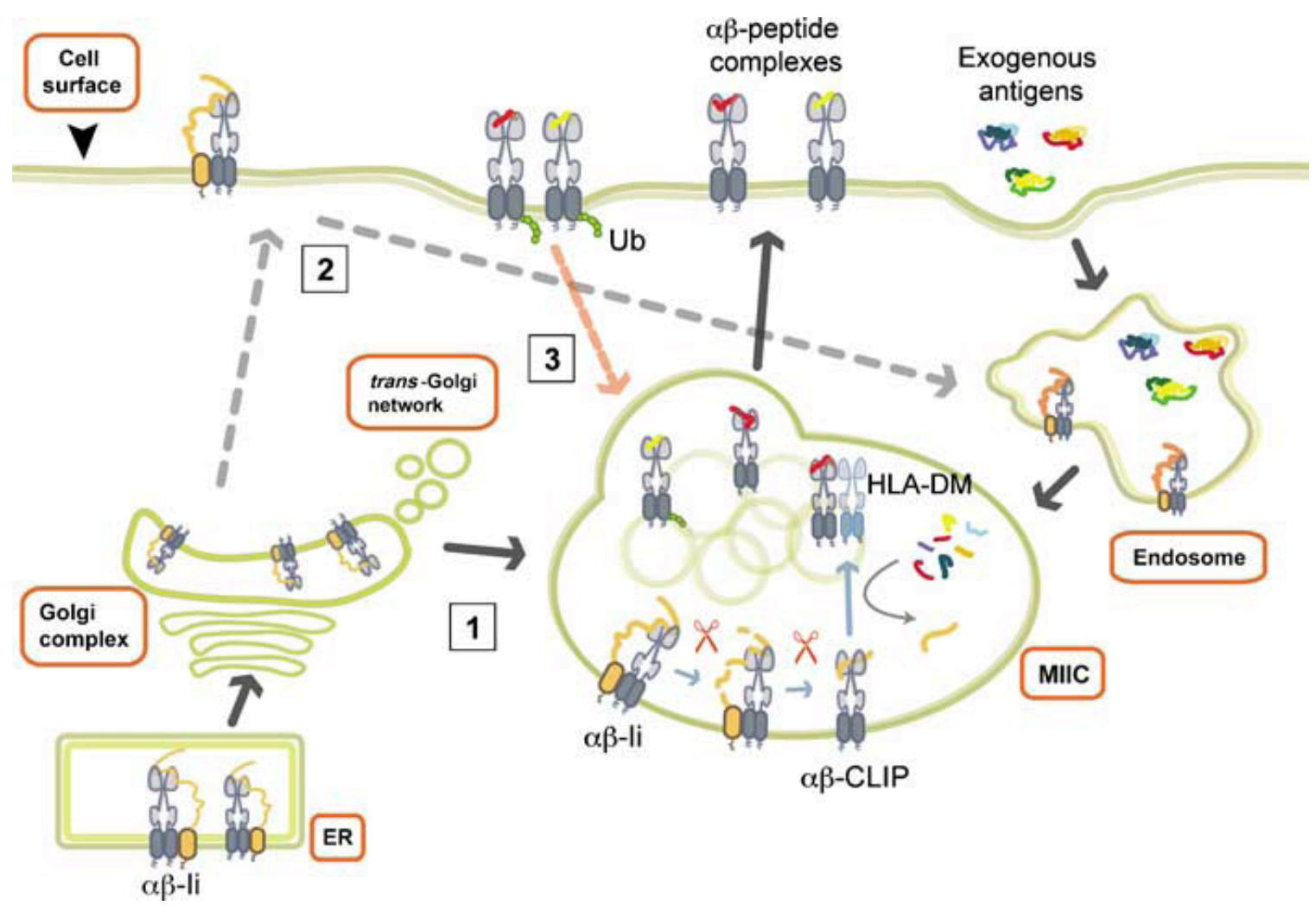

Figure 3.1: Overview on the MHCII antigen presentation pathway.

Exogenous antigens are taken up by professional APCs and localise to endosomes, in which processing to peptides takes place. The peptides are delivered to the MIIC compartment. Newly synthesised MHCII molecules assemble in the ER and associate with the invariant chain $(\alpha \beta-\mathrm{Ii})$, which prevents premature acquisition of ER-resident peptides and directs MHCII molecules to the MIIC compartment via the TGN. The Ii is sequentially cleaved by cathepsins and HLA-DM in the MIIC, so that MHCII molecules can acquire antigenic peptides. Peptide-loaded MHCII molecules are then transported to the cell surface. Cell-surface MHCII is constitutively internalised and locates to endosomes, from where it can be transported to MIIC for re-acquisition of new peptides Adopted from ref. (93). 
In the MIIC, the Ii is sequentially cleaved by acidic proteases such as cathepsins, and peptides are loaded onto MHCII molecules. Peptide loading is catalysed by $\mathrm{H}-2^{\mathrm{m}}$ in the mouse (HLA-DM in humans), an MHCII-like molecule that predominantly localises to MIIC and is not expressed on the cell surface (93). Another function of $\mathrm{H}-2^{\mathrm{m}}$ is the release of weakly bound peptides from MHCII, which ensures that MHCII molecules are transported to the cell surface only with stably bound peptides (93).

The final step in antigen processing and presentation is the transport of pMHCII complexes from the MIIC to the cell surface. The complexes are transported within vesicles, and the directed transport to the cell surface is mediated by microtubules and associated motor proteins (6). At the cell surface, pMHCII can be recognised by specific TCRs on CD4 T cells (6).

Peptide binding to MHCII is usually irreversible, but pMHCII are constitutively internalised from the cell surface (94). Internalised pMHCII localise to endocytic vesicles, where peptides are removed. MHCII molecules can subsequently be transported to MIIC compartments, for re-loading with new antigenic peptides (figure 3.1). This results in presentation of newly acquired peptides by recycled MHCII on the surface of the APC, rather than solely depending on a supply of synthesised MHCII (94).

\subsubsection{Cross-presentation}

Professional APCs, and DCs in particular, have the capacity to take up and degrade extracellular pathogen-derived compounds or cell fragments, deliver peptides to the MHCI antigen presentation pathway and present them to CD8 T cells, a process known as cross-presentation (95). This feature of the immune system allows priming of CD8 T cells to combat pathogens that do not infect APCs.

The mechanism of cross-presentation and the route antigens take can vary, depending on the nature of the antigen and the type of cross-presenting cell (96). Most studies have found that endocytosed antigens are required to escape the endocytic pathway and be degraded by the proteasome in the cytosol $(95,96)$. The antigens are subsequently transported into the ER by the transporter associated with antigen processing (TAP) complex. In the ER, loading of antigenic peptides onto newly synthesised MHCI molecules occurs, similar to the loading of intracellular pathogenderived antigens. However, it is not clear how material contained in endocytic vesicles gains access to the cytosol across membranes (96). 
Recent reports have shown that internalised virions can be processed and the resulting peptides loaded on MHCI molecules in early endocytic vesicles of plasmacytoid DCs in a proteasome-independent manner (97). These vesicles may represent a specialised compartment for processing and cross-presentation of antigens derived from the cell exterior. Soluble antigens can also be diverted to the cross-presentation pathway in early endosomes. This requires their processing by proteasomes and re-import into endosomes by the TAP complex, which is recruited to endosomal membranes through TLR4 signalling (98).

Research on the mechanisms of cross-presentation has been of great impact, because manipulation of this pathway may prove useful in the development of improved vaccines against viral infections and tumours, the challenge of which require a robust CD8 T cell response (99).

\subsubsection{Superantigens}

Superantigens (SAgs) are toxins that are predominantly produced by the grampositive bacteria and , as well as by several other bacteria and viruses (100). Bacterial SAgs are secreted monomeric proteins with a molecular mass of $24-28 \mathrm{kDa}(101)$. The benefit of SAgs to the bacteria that produce them is not fully understood. However, SAgs can have substantial effects on the immune system and cause severe pathologies like toxic shock or food poisoning (100).

SAgs have the ability to simultaneously bind to MHCII molecules and to TCRs. In this way, SAgs are able to induce polyclonal $\mathrm{T}$ cell expansion and systemic release of inflammatory cytokines (102). Unlike conventional peptide antigens, SAgs can bind directly to MHCII and the TCR V $\beta$ domain without being processed (103). In contrast to the highly specific interaction between TCRs and pMHCII complexes, SAgs are able to antigen non-specifically activate any $\mathrm{T}$ cell that expresses the correct TCR V $\beta$ segment on its surface (103). As a result, a specific pMHCII complex activates an estimated one in $10^{5}$ to $10^{6} \mathrm{~T}$ cells, where up to $20 \%$ of total $\mathrm{T}$ cells can be reactive to a given $\mathrm{SAg}(100)$.

Streptococcal mitogenic exotoxin Z (SMEZ) is one of the most potent SAgs discovered to date (104). It has been found to be highly polymorphic, and more than 20 allelic variants have been discovered from clinical isolates in New Zealand (105). A strain-specific variant, SMEZ2, has been recovered from strain 2035, based on sequence homology to the original SMEZ (101). 
SMEZ2 M1 is a modified form of SMEZ2 that carries mutations in three amino acid residues (W75L, K182Q, D42C) that are responsible for TCR binding (106). As a result of these mutations, SMEZ2 M1 maintains the ability to bind MHCII without the toxic effects associated with the wild-type SAg.

\subsubsection{Objectives}

The first objective of the experiments presented in this chapter was to establish a GA treatment model of EAE suppression. Different GA formulations, sites of administration and doses were tested in order to set up a consistent EAE therapy. The use of a reliable model of GA treatment was of crucial importance for the subsequent assessment of drug targeting strategies.

The main objective was to use the GA treatment model to develop a targeting strategy, aiming at the direct delivery of GA to MHCII molecules on the surface of APCs. For this, two different targeting molecules were used: a monoclonal antibody recognising MHCII or a modified SAg (SMEZ2 M1) that can also bind to MHCII with high affinity. This targeting strategy was hypothesised to significantly enhance uptake of GA by APCs and its presentation to CD4 T cells, resulting in an enhancement of GAspecific $\mathrm{T}$ cell responses and an increase in the drug's efficiency.

Prior to use in EAE treatment, the targeting strategy was validated by proof-of-concept experiments, in which expansion of TCR transgenic T cells was measured after incubation with MHCII-targeted cognate antigen. 


\subsection{Results}

\subsubsection{Establishment of a GA Treatment Model of EAE Suppression}

\subsubsection{Pre-treatment with $G A$ in PBS does not suppress $E A E$}

In the EAE model that was used throughout this study, disease was induced in C57BL/6J mice by subcutaneous immunisation with $50 \mu \mathrm{g} \mathrm{MOG}_{35-55}$ emulsified in CFA in the hind limb flanks. The average disease onset was apparent 10-12 days after EAE induction (figure 3.2). Untreated mice developed severe disease (EAE score 3-4) within four to five days after onset, and maintained clinical symptoms until the end of the 40-day observation period. In some experiments a slight decrease of the clinical score was observed following the initial phase of severe disease, but total remittance or recovery did not occur (figure 3.3).

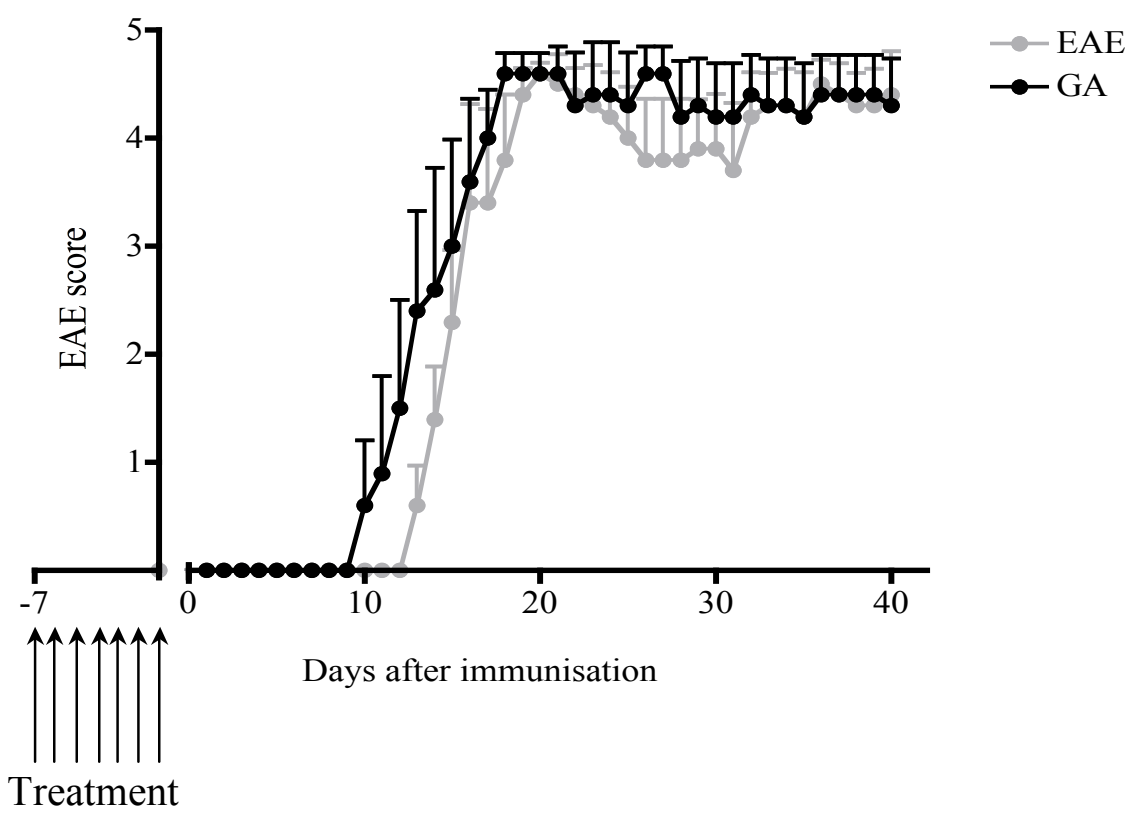

Figure 3.2: Pre-treatment with GA in PBS does not suppress EAE.

C57BL/6J mice received subcutaneous injections of $150 \mu \mathrm{g}$ GA in PBS in the neck on seven consecutive days before EAE induction. Control mice (EAE) were injected with PBS only. ( $p>0.05$ for EAE vs. GA; mean \pm SEM of $n=5$ mice per group). Data represents one out of two independent experiments. 
In order to establish a GA treatment model of EAE suppression, first a GA formulation was tested that did not contain adjuvants. Mice received subcutaneous injections of $150 \mu \mathrm{g}$ GA in PBS into the scruff of the neck on seven consecutive days preceding EAE induction. As shown in figure 3.2, this mode of GA treatment did not have any effect on EAE onset or progression. Hence, subcutaneous injection of GA in PBS in the neck before disease induction was not an effective treatment for EAE.

\subsubsection{Co-Immunisation with $M_{0} G_{35-55}$ and GA Suppresses EAE}

In further effort to establish a GA treatment model, another GA formulation was tested that contained CFA as an adjuvant. EAE was induced in control mice as described above. For treatment, GA and $\mathrm{MOG}_{35-55}$ were mixed with $\mathrm{CFA}$ and subcutaneously injected into the hind limb flanks as a single emulsion that contained both the diseasecausing peptide and the disease-suppressing drug (107). As shown in figure 3.3, this GA formulation effectively suppressed EAE. This "co-immunisation" treatment was used as a positive control for EAE suppression for the rest of this study.

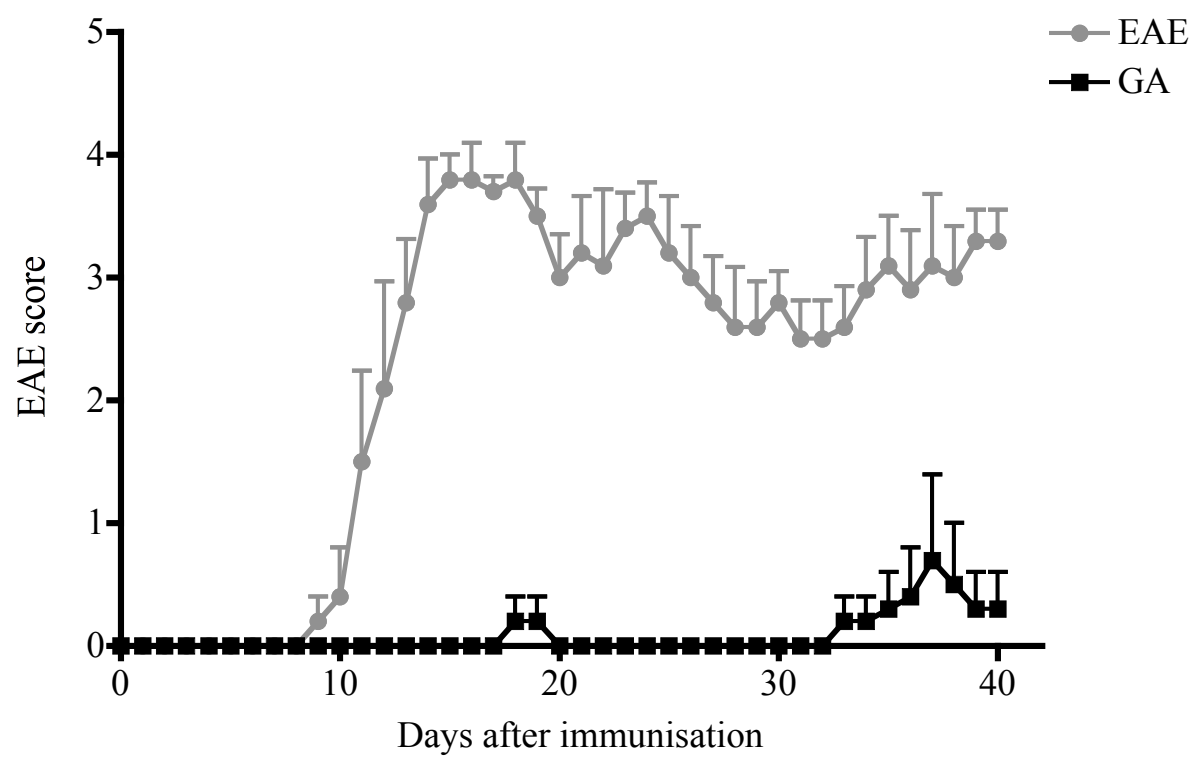

Figure 3.3: Co-immunisation with $\mathrm{MOG}_{35-55}$ and $\mathrm{GA}$ in CFA suppresses EAE.

C57BL/6J mice received a single subcutaneous immunisation with $50 \mu \mathrm{g} \mathrm{MOG}_{35-55}$ with or without $500 \mu \mathrm{g}$ GA in CFA. ( $p<0.001$ for EAE vs. GA; mean \pm SEM of $\mathrm{n}=5$ mice per group). Data represents one out of twelve independent experiments. 
At the end of the 40-day observation period spinal cords were removed from coimmunised and untreated mice and sections of the spinal cords were stained with hematoxylin and eosin (figure 3.4). Local cellular infiltration of the spinal cords was evident in the sections from untreated mice, but no such infiltrations could be detected in the sections from the GA treated mice.

Untreated
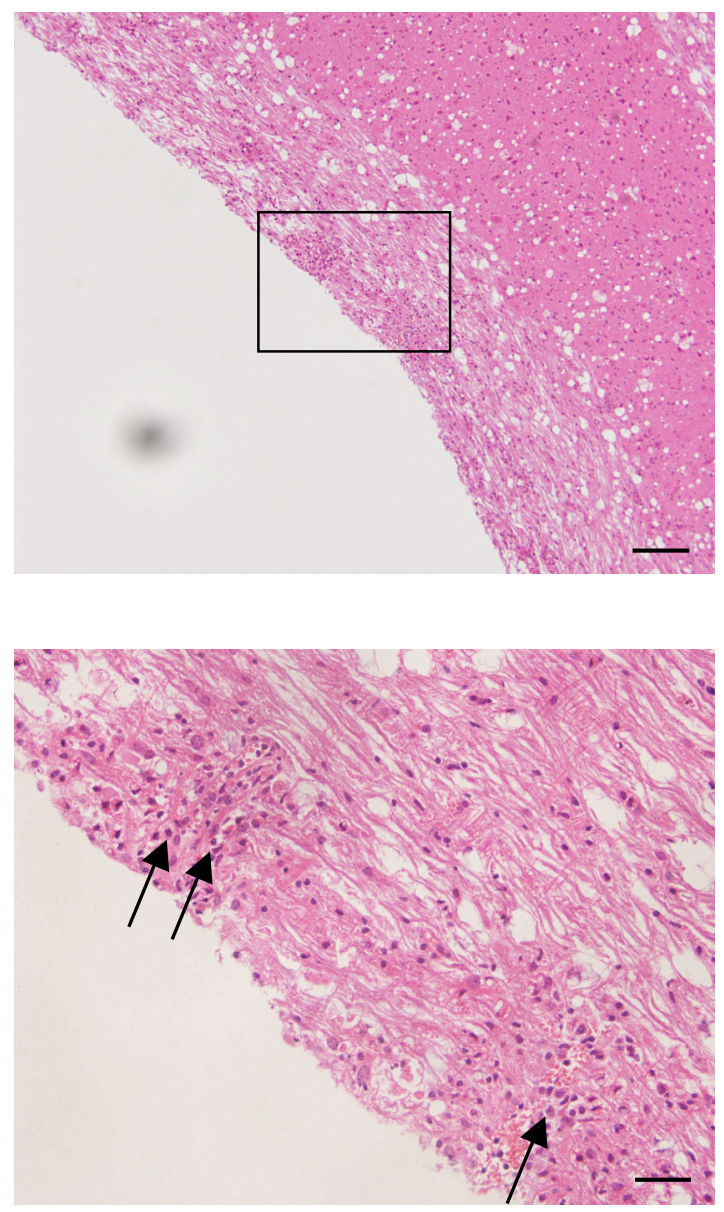

GA treated
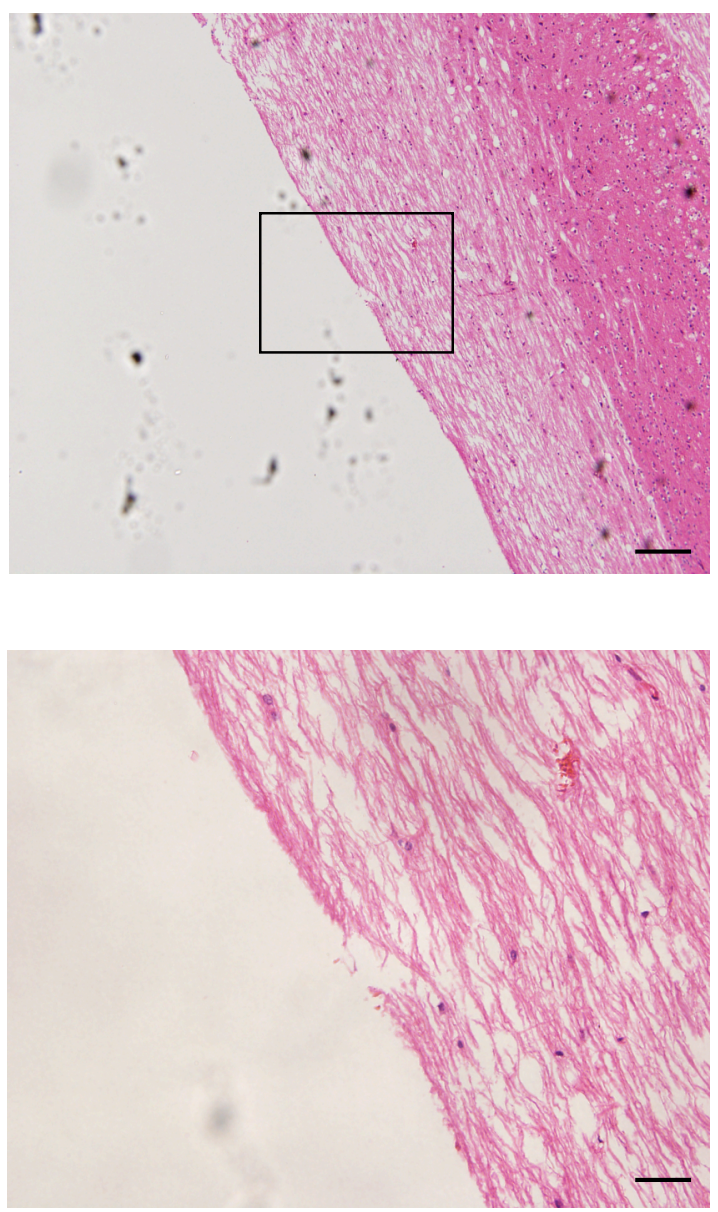

Figure 3.4: GA treatment prevents cellular infiltration of the spinal cord.

EAE was induced in C57BL/6J mice, and the mice were either left untreated or coimmunised with $500 \mu \mathrm{g}$ GA. Spinal cords were removed 40 days after immunisation and paraffin-embedded $6 \mu \mathrm{m}$ sections were stained with hematoxylin and eosin. Original magnification is $100 \mathrm{x}$ in upper panels. Lower panels show boxed area at $400 \mathrm{x}$ magnification. Scale bars: $200 \mu \mathrm{m}$ (upper panels) or $50 \mu \mathrm{m}$ (lower panels). Arrows: Clusters of infiltrating cells. Images shown are representative for three individual mice per group from one EAE experiment. 
Co-immunisation with $500 \mu \mathrm{g}$ GA almost completely suppressed EAE in most of the experiments. To determine whether EAE could be efficiently suppressed by lower doses of GA, mice were treated by co-immunisation with decreasing doses. As shown earlier, $500 \mu \mathrm{g}$ GA was very efficient in suppressing EAE (figure 3.5). Reduction of the dose to $250 \mu \mathrm{g}$ resulted in a statistically significant loss of protection. Mice treated with $100 \mu \mathrm{g}$ GA developed slightly more severe EAE than mice treated with $250 \mu \mathrm{g}$ GA. Finally, $50 \mu \mathrm{g}$ GA lacked significant protective effects (figure 3.5). These findings showed that $500 \mu \mathrm{g}$ GA were necessary to achieve efficient EAE suppression by coimmunisation treatment.

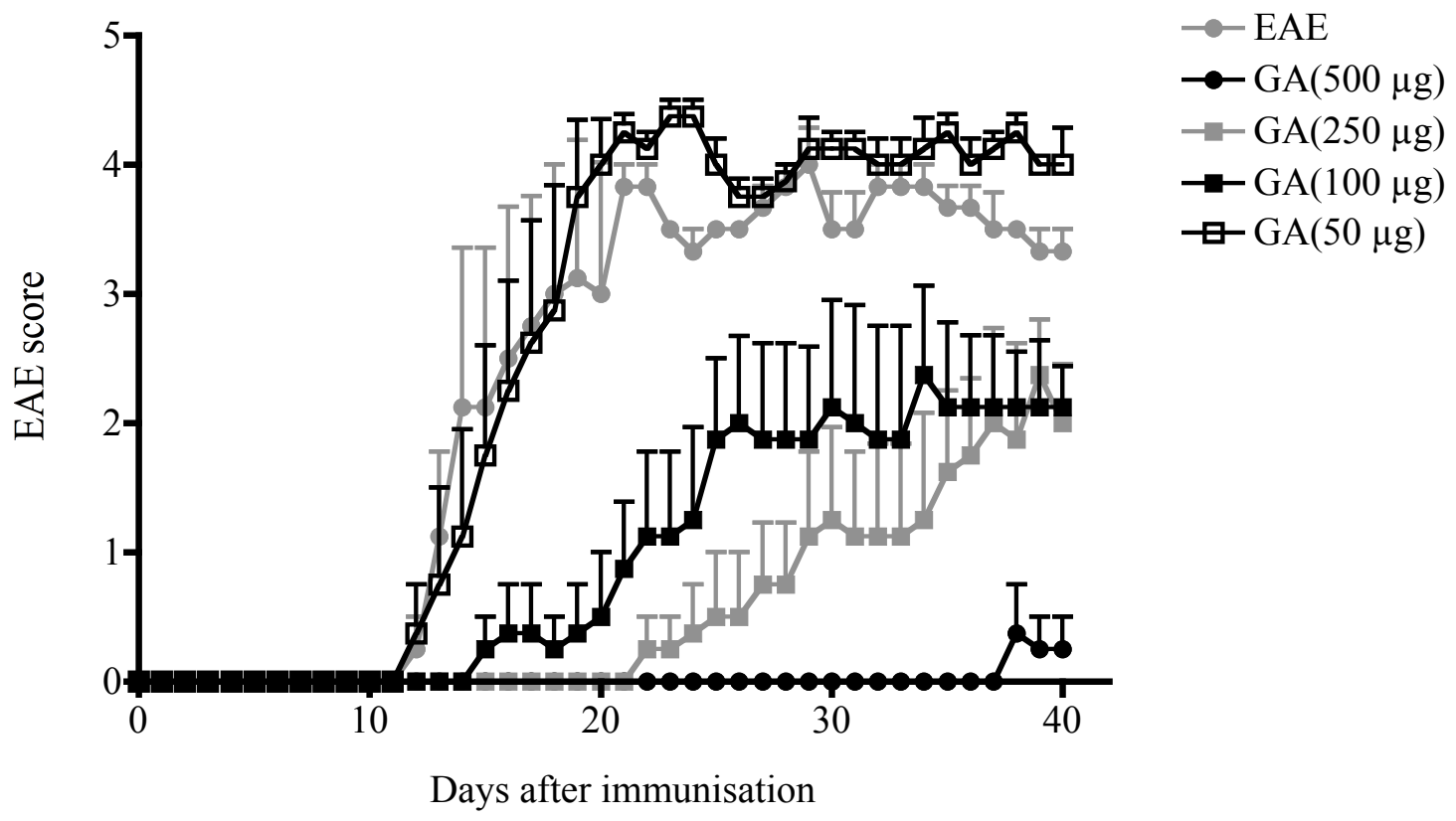

Figure 3.5: Determination of the optimal dose of GA for efficient EAE suppression. C57BL/6J were treated by co-immunisation with the indicated doses of GA. Control mice (EAE) were left untreated. ( $\mathrm{p}<0.05$ for GA $(500 \mu \mathrm{g})$ vs. GA $(250 \mu \mathrm{g}), \mathrm{p}>0.05$ for EAE vs. GA $(50 \mu \mathrm{g})$; mean $\pm \mathrm{SEM}$ of $\mathrm{n}=5$ mice per group). Data represents one out of two independent experiments.

The different results obtained from treatment experiments raised the question whether the choice of the treatment site was important for efficient EAE suppression. To address this question, EAE was induced as described above and mice were treated with $500 \mu \mathrm{g}$ GA emulsified in CFA subcutaneously in the scruff of the neck immediately after disease induction. Mice that had been treated in the neck developed slightly less severe 
EAE than the untreated mice, but this difference did not reach statistical significance (figure 3.6). Disease onset was similar in treated and untreated mice. Mice treated by coimmunisation were better protected from EAE than mice that had been treated in the neck, suggesting that the treatment site may be important for protection from EAE.

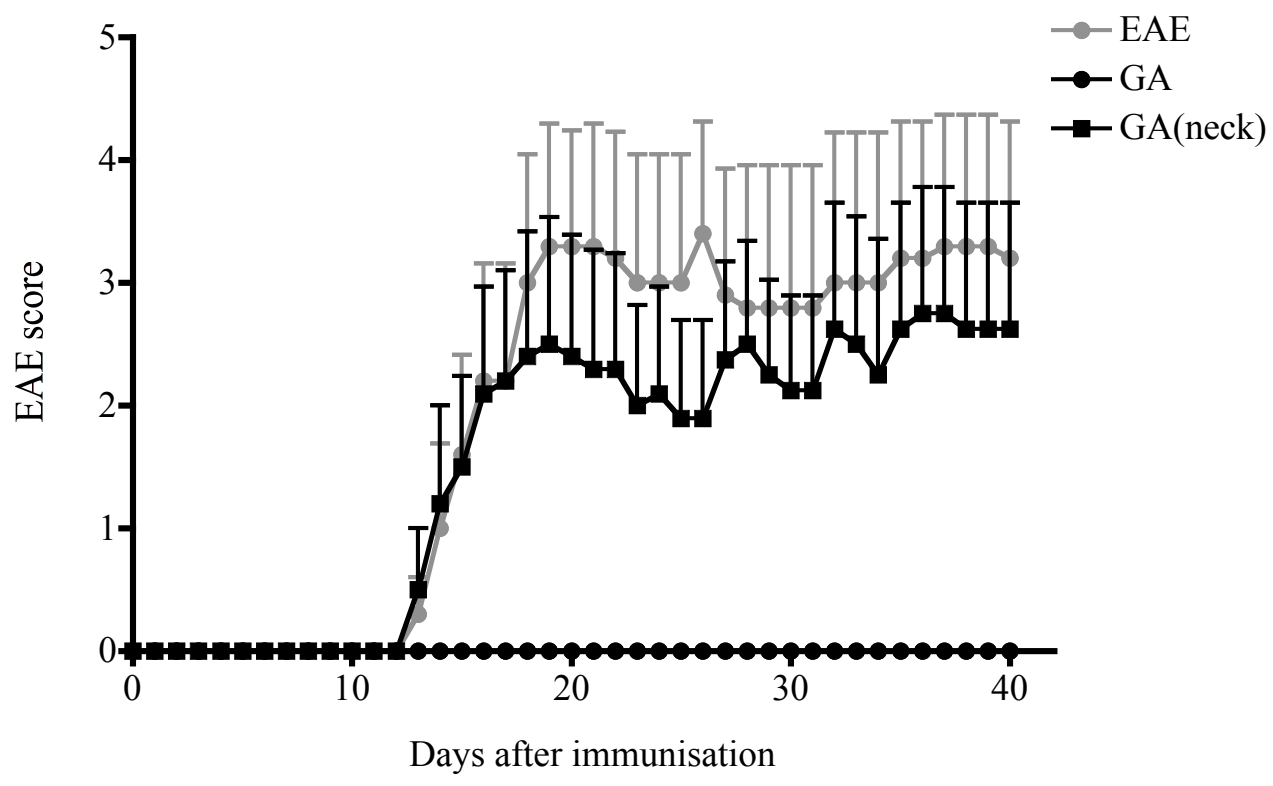

Figure 3.6: Co-immunisation treatment is superior to GA treatment in the neck.

C57BL/6J were either treated by subcutaneous co-immunisation or subcutaneous injections of $500 \mu \mathrm{g} \mathrm{GA}$ in CFA in the neck. Control mice (EAE) were left untreated. $(\mathrm{p}<0.001$ for GA vs. GA (neck), $\mathrm{p}>0.05$ for EAE vs. GA (neck); mean \pm SEM of $\mathrm{n}=5$ mice per group).

\subsubsection{Mannitol is not Required for Effective Co-Immunisation Treatment}

The formulation of GA that is approved for MS therapy and that was used in previous experiments contains $40 \mathrm{mg} / \mathrm{ml}$ mannitol as a stabilising agent. Strategies of GA targeting involve the chemical conjugation of GA to targeting molecules. The conjugation process includes several steps of desalting; hence the GA conjugates do not contain mannitol. Therefore, it was tested whether the absence of mannitol from the therapeutic GA formulation altered the efficacy of EAE treatment.

Mannitol was removed from the GA solution by the use of size exclusion columns with a molecular weight cut-off of $3 \mathrm{kDa}$. The filtrate was discarded and the remaining 
amount of GA in the concentrate was determined by $\mathrm{OD}_{280}$ measurement. Mice were then treated by co-immunisation with $500 \mu \mathrm{g}$ GA without mannitol. As shown in figure 3.7, mice treated with GA with or without mannitol showed a similar extent of protection from EAE. Some of the mice treated with the mannitol-containing GA formulation developed mild EAE from day 25 after EAE induction, while the mice treated with mannitol-free GA were completely protected. However, the difference between the two treatment groups was not statistically significant. This finding showed that therapeutic effect of GA in EAE treatment did not depend on the presence of mannitol.

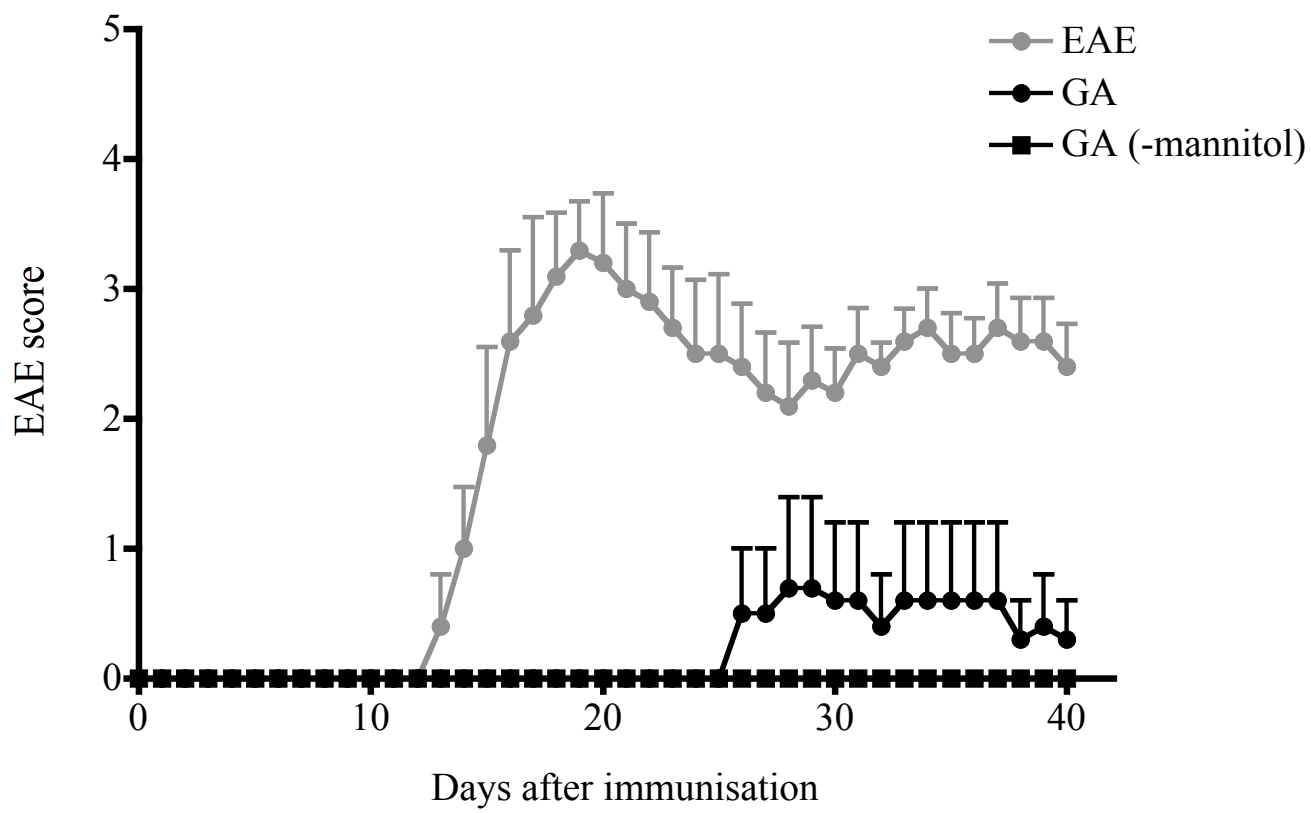

Figure 3.7: Therapeutic effect of GA does not depend on the presence of mannitol. C57BL/6J received EAE induction and were co-immunised with two different GA formulations, either with or without mannitol ( $p<0.001$ for EAE vs. GA (-mannitol), $\mathrm{p}>0.05$ for GA vs. GA (-mannitol); mean \pm SEM of $\mathrm{n}=5$ mice per group). Data represents one out of two independent experiments.

\subsubsection{GA does not Suppress EAE in $M_{30 G_{35-55}}$ TCR Transgenic Mice}

To determine whether suppression of EAE by GA was dependent on $\mathrm{T}$ cell recognition of GA peptides presented on MHCII molecules, the co-immunisation treatment was tested in mice that express a transgenic TCR that is not reactive to GA.

2D2 mice express a transgenic TCR on their CD4 $\mathrm{T}$ cells that is specific for $\mathrm{MOG}_{35-55}$ (92). 2D2 mice developed more severe EAE compared to their wild-type 
counterparts, and all of the mice were euthanised between days 20-30 after disease induction due to severe EAE (figure 3.8). Reduction of the dose of $\mathrm{MOG}_{35-55}$ from $50 \mu \mathrm{g}$ to $25 \mu \mathrm{g}$ for EAE induction did not improve EAE severity or survival of 2D2 mice (see A1.1). In this 2D2 model of EAE, co-immunisation with $500 \mu \mathrm{g}$ of GA failed to ameliorate disease or increase survival (figure 3.8), suggesting that $\mathrm{T}$ cell recognition of GA peptides presented on MHCII molecules is key for EAE suppression.

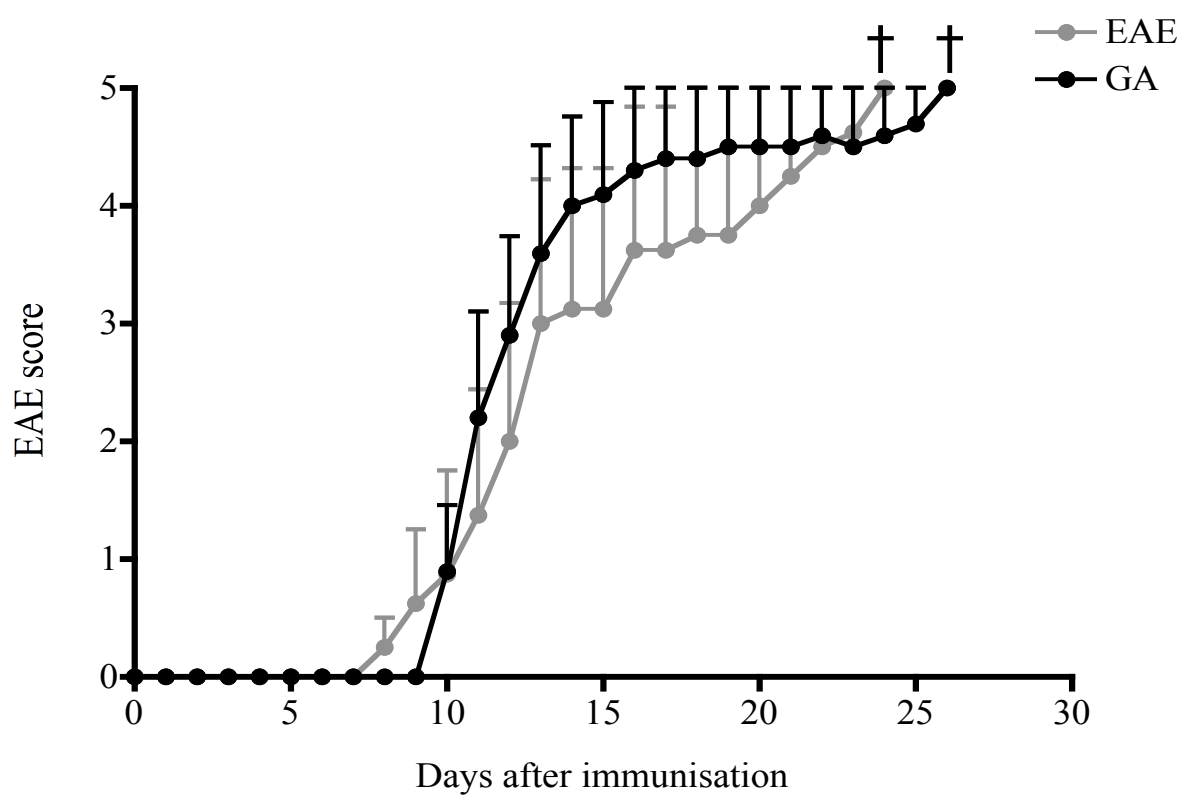

Figure 3.8: GA does not suppress EAE in $\mathrm{MOG}_{35-55}$ TCR transgenic mice.

EAE was induced in 2D2 mice with $50 \mu \mathrm{g} \mathrm{MOG}_{35-55}$. Mice were either left untreated (EAE) or co-immunised with $500 \mu \mathrm{g}$ GA. ( $p>0.05$ for EAE vs. GA; mean \pm SEM of $\mathrm{n}=5$ mice per group). Data represents one out of three independent experiments. $†$ indicates that all mice in the corresponding group were euthanised due to severe EAE.

In summary, a GA treatment model of EAE suppression was established in C57BL/6J mice. In this model mice were subcutaneously co-immunised with the disease causing MOG peptide and GA in a single CFA emulsion in the hind limb flanks. The treatment was most effective at a dose of $500 \mu \mathrm{g}$ GA and did not depend on the presence of mannitol. Although effective in wild-type mice, co-immunisation treatment failed to suppress EAE in transgenic mice that express a $\mathrm{MOG}_{35-55}$ specific TCR and cannot mount GA-specific T cell responses. The co-immunisation treatment was used in the treatment of wild-type mice throughout the rest of this study in the investigation of drug targeting strategies. 


\subsubsection{Antigen Targeting to MHCII with a Specific Antibody in vitro}

The aim of targeting GA into the MHCII antigen presentation pathway was to increase the efficacy of the drug by enhancing its uptake and presentation. Being a random copolymer, GA lacks a defined primary sequence, and can give rise to a range of different MHCII binding peptides (42). As a consequence, TCR transgenic systems specific for GA are not available. For this reason, a series of proof-of-concept experiments was performed using MOG and OVA proteins and splenocytes from the respective TCR transgenic 2D2 and OT-II mice.

\subsubsection{Conjugation of MOG and OVA Proteins to an anti-MHCII Antibody}

Due to the apparent importance of MHCII-restricted presentation of GA peptides to CD4 T cells during GA therapy, a monoclonal antibody specific for MHCII of the H-2 ${ }^{\mathrm{b}}$ haplotype (clone M5/114) was chosen as targeting molecule. For conjugation of proteins to M5/114, a two-component chemical conjugation system was used. The conjugation process involved the modification of the antibody and the protein with the two chemicals separately, followed by the co-incubation of the modified antibody and protein. The two chemicals (C6-S-HyNic and sulfo-S-4FB) react with primary amines, i.e. with lysine residues and the $\mathrm{N}$-terminal amine group of peptide backbones, and are complementary to each other, so that homotypic conjugation does not occur (108). The use of a single heterobifunctional amine/cysteine-reactive chemical was not feasible, because it would require the reduction of antibodies, which would in turn result in the loss of its quaternary structure and consequently in the loss of its function.

Different experimental conditions were tested in order to optimise the conjugation protocol. Variables were the molar ratio of M5/114 to C6-S-HyNic (HyNic hereafter), the ratio of MOG or OVA to the complementary sulfo-S-4FB (4FB hereafter), and the ratio of the modified antibody to protein during co-incubation. Efficiency of conjugation was evaluated by non-reducing SDS-PAGE. A summary of the optimisation of the M5/114-MOG conjugation is given in table 3.1, and a representative SDS gel picture of the conjugates is shown in figure 3.9. The optimal reaction conditions were found to be the following for M5/114-MOG: Ratio (M5/114:HyNic) 1:100; ratio (MOG:4FB) 1:5; ratio (M5/114:MOG) 1:30. The conditions for the optimal conjugation of M5/114 to OVA were similar, except that the ratio (OVA:4FB) was 1:20 in this case. 
Table 3.1: Optimisation of the conjugation of M5/114 to MOG.

Correlation of molar ratios of antibody or protein to the respective chemical used for modification, as well as the molar ratios of antibody to protein with the efficacy of conjugation. Representative SDS gel images of "poor", "fair" and "good" conjugation reactions are shown in figure 3.9.

\begin{tabular}{|c|c|c|c|}
\hline M5/114:HyNic & MOG:4FB & M5/114:MOG & Outcome \\
\hline $1: 20$ & $1: 20$ & $3: 1$ & Poor conjugation \\
\hline $1: 20$ & $1: 20$ & $1: 1$ & Poor conjugation \\
\hline $1: 20$ & $1: 20$ & $1: 3$ & Poor conjugation \\
\hline $1: 20$ & $1: 20$ & $1: 10$ & Poor conjugation \\
\hline $1: 20$ & $1: 20$ & $1: 20$ & Poor conjugation \\
\hline $1: 20$ & $1: 20$ & $1: 50$ & Poor conjugation \\
\hline $1: 20$ & $1: 20$ & $1: 100$ & Poor conjugation \\
\hline $1: 10$ & $1: 5$ & $1: 3$ & Poor conjugation \\
\hline $1: 10$ & $1: 5$ & $1: 30$ & Fair conjugation \\
\hline $1: 10$ & $1: 20$ & $1: 3$ & Poor conjugation \\
\hline $1: 10$ & $1: 20$ & $1: 30$ & Poor conjugation \\
\hline $1: 100$ & $1: 5$ & $1: 3$ & Poor conjugation \\
\hline $1: 100$ & $1: 5$ & $1: 30$ & Good conjugation \\
\hline $1: 100$ & $1: 20$ & $1: 3$ & Poor conjugation \\
\hline $1: 100$ & $1: 20$ & $1: 30$ & Fair conjugation \\
\hline $1: 100$ & $1: 5$ & $1: 15$ & Fair conjugation \\
\hline
\end{tabular}

Conjugates of a molecular weight of $>205 \mathrm{kDa}$ were frequently observed (figure 3.9). The binding of a large number of proteins to one antibody molecule is not a satisfactory explanation for this observation, because high molecular weight (MW) complexes were observed in clusters over a relatively narrow range of molecular weight. Rather, the high-MW complexes probably consisted of two or more antibodies interconnected by protein molecules.

In almost every permutation of reaction conditions tested, there was excess MOG or OVA protein left over in the reaction mixture that did not conjugate to M5/114 (see figure 3.9). Overall, reaction conditions with low amounts of unconjugated antibody were favoured over those with low amounts of unconjugated protein. The reason for this was that the removal of excess protein from the conjugates by size exclusion or gel filtration was anticipated to be less challenging than the removal of excess antibody. 


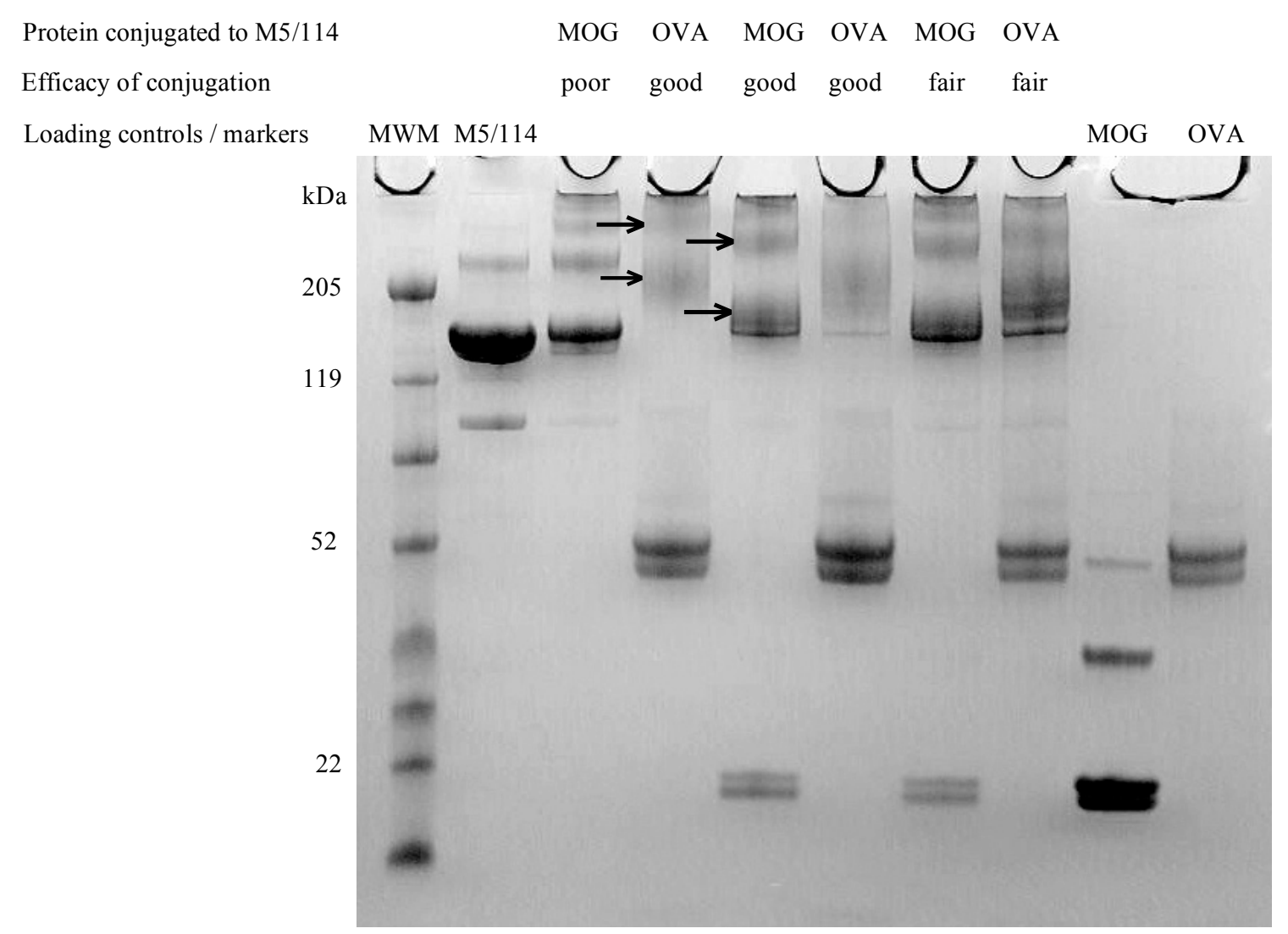

Figure 3.9: Optimisation of the conjugation of MOG and OVA proteins to M5/114.

The effect of varying reaction conditions on the efficacy of conjugation of MOG or OVA to M5/114 was evaluated. MOG or OVA were conjugated to M5/114 under varying conditions (see table 3.1), and efficiency of conjugation was assessed by non-reducing SDS-PAGE and Coomassie blue staining. M5/114 and MOG and OVA proteins were loaded alone as controls. The location of conjugates on the gel is exemplified in two lanes by arrows. MWM: molecular weight marker.

Several different chromatographic approaches were tested for the removal of excess protein from the conjugates (table 3.2). The use of self-packed gel filtration columns did not result in satisfactory separation of the conjugates from any of the free proteins tested. For removal of excess MOG, both mini-centrifuge size-exclusion columns (50 kDa MW cut-off) and Ig-based affinity chromatography (HiTrap Protein G columns) were effective. However, for removal of OVA, neither of these methods was suitable. The use of self-packed affinity columns that contained an antibody specific for rat IgG $\kappa$ chains (clone MAR18.5) also failed to bind to M5/114-OVA and therefore could not be used to separate the conjugate from free OVA (table 3.2). A control mixture 
of unconjugated M5/114 and OVA could be effectively separated with both affinity columns.

Table 3.2: Purification of M5/114-antigen conjugates by chromatography.

MOG, OVA and GA were conjugated to M5/114. The indicated chromatography columns were used to separate the conjugates from excess free protein (see Materials and Methods). The outcome of the different means of purification is stated.

\begin{tabular}{|c|c|c|c|}
\hline Conjugate & Purification method & Column & Outcome \\
\hline M5/114-MOG & Gel filtration & Sephacryl 300 HR & Poor purification \\
\hline M5/114-MOG & Size exclusion & Vivaspin 50K MWCO & Good purification \\
\hline M5/114-MOG & Affinity & HiTrap Protein G & Good purification \\
\hline M5/114-OVA & Gel filtration & Sephacryl 300 HR & Poor purification \\
\hline M5/114-OVA & Size exclusion & Vivaspin 50K MWCO & No purification \\
\hline M5/114-OVA & Affinity & HiTrap Protein G & No purification \\
\hline M5/114-OVA & Affinity & CNBr Sepharose-MAR18.5 & No purification \\
\hline M5/114-GA & Size exclusion & Vivaspin 50K MWCO & Poor purification \\
\hline M5/114-GA & Affinity & HiTrap Protein G & Good purification \\
\hline
\end{tabular}

In summary, the experimental procedure for conjugation of MOG and OVA proteins to M5/114 was optimised. Excess unconjugated MOG protein was effectively separated from the conjugates, and the M5/114-MOG conjugate was then tested for its potential to induce $\quad T$ cell proliferation (section 3.2.2.2). Excess OVA could not be removed, and the conjugate was therefore used as a mixture of M5/114-OVA and OVA in the proliferation assays.

\subsubsection{Targeting MOG and OVA to MHCII Enhances CD4 T Cell Proliferation in vitro}

$\mathrm{T}$ cell proliferation assays were performed using M5/114-MOG and splenocytes from TCR transgenic 2D2 mice. MOG protein was conjugated to M5/114 and M5/114-MOG was separated from excess MOG as described in the previous section (figure 3.10A). Concentration of M5/114-MOG was determined by $\mathrm{OD}_{280}$ measurement. The molecular weight (MW) of M5/114-MOG was considered to be $175 \mathrm{kDa}$ on average, as estimated from gel pictures (figure 3.10A). 

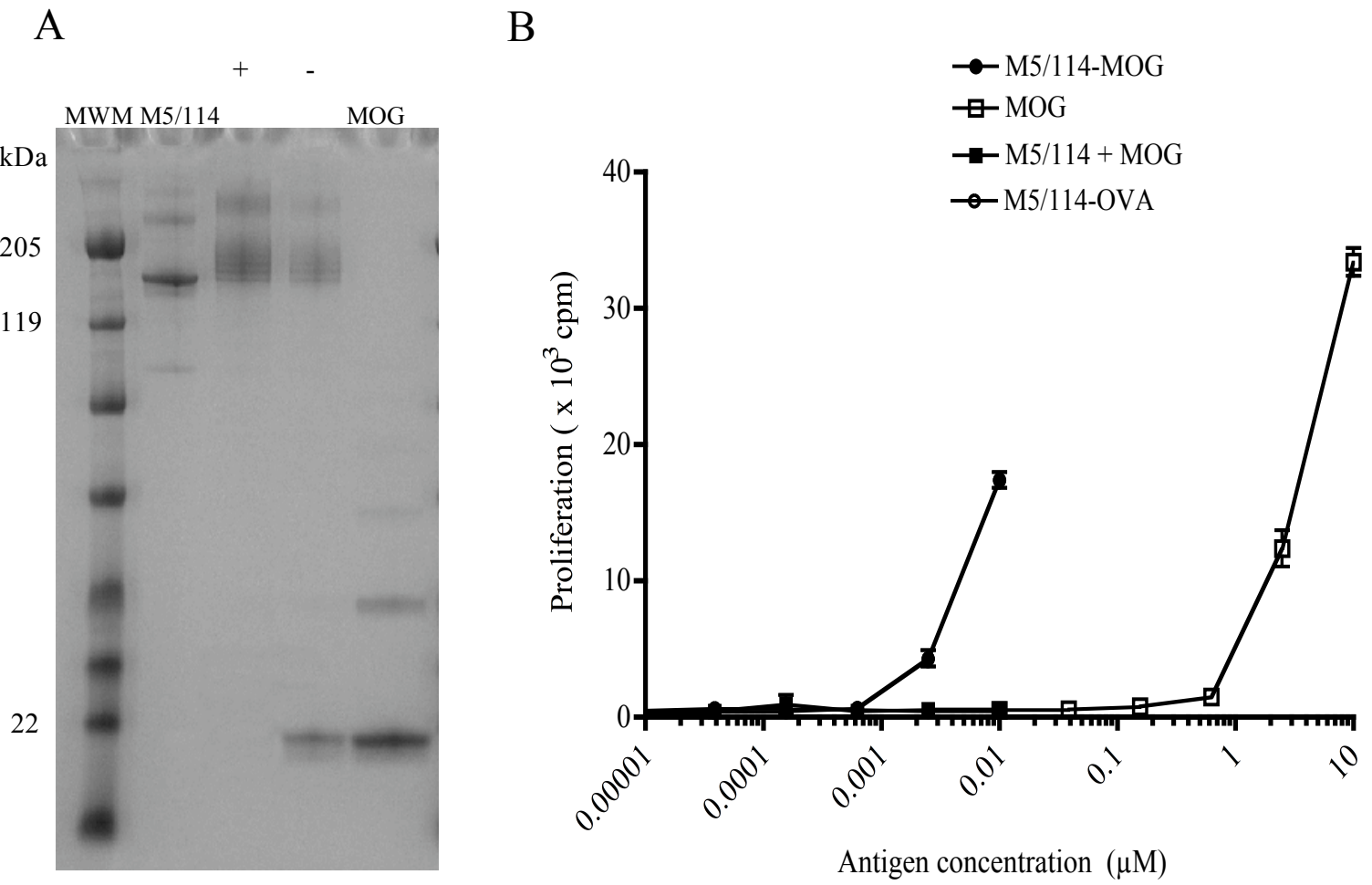

Figure 3.10: Targeting MOG to MHCII enhances CD4 $\mathrm{T}$ cell proliferation in vitro.

(A) MOG protein was conjugated to M5/114 and the conjugates purified with Protein G columns as described in Materials and Methods. The purified (+) and unpurified (-) conjugates as well as loading controls were analysed by non-reducing SDS-PAGE and stained with Coomassie blue. MWM: Molecular weight marker. (B) Proliferation assay with splenocytes from 2D2 mice. Splenocytes were incubated with the indicated concentration of antigens at $5 \times 10^{5}$ cells per well. The cells were incubated for 72 hours, and eight more hours in presence of $\left[{ }^{3} \mathrm{H}\right]$-thymidine. Proliferation was measured by the incorporation of $\left[{ }^{3} \mathrm{H}\right]$-thymidine. Data represents one out of five independent experiments (mean \pm SEM of triplicate wells).

Purified M5/114-MOG was incubated with 2D2 splenocytes to compare the proliferative response to free MOG. As shown in figure 3.10B, M5/114-MOG stimulated CD4 T cell proliferation at more than 100-fold lower concentrations than free MOG. A mixture of unconjugated M5/114 and MOG failed to induce proliferation at the same concentration as M5/114-MOG. As expected, M5/114-OVA failed to stimulate MOGspecific T cells (figure 3.10B). 
M5/114-OVA (estimated average MW $190 \mathrm{kDa}$, figure 3.11A) stimulated CD4 T cells at an almost 1000-fold lower concentration than free OVA (figure 3.11B). Although the conjugates could not be separated from excess OVA (figure 3.11A), a mixture of unconjugated M5/114 and free OVA failed to induce T cell proliferation at comparable doses to M5/114-OVA (figure 3.11B), indicating that the free OVA in the conjugation mixture was unlikely to contribute to the observed $\mathrm{T}$ cell proliferation in response to M5/114-OVA. The enhanced $T$ cell expansion induced by M5/114-MOG and M5/114-OVA showed that uptake of selected antigens can be enhanced by conjugating them to an anti-MHCII antibody, resulting in markedly enhanced capacity of APCs to induce proliferation of CD4 T cells.

A

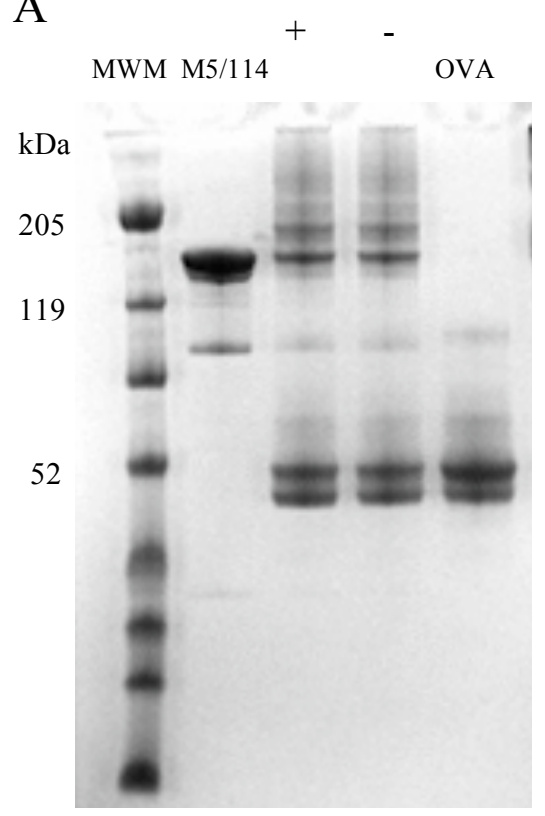

$\mathrm{B}$

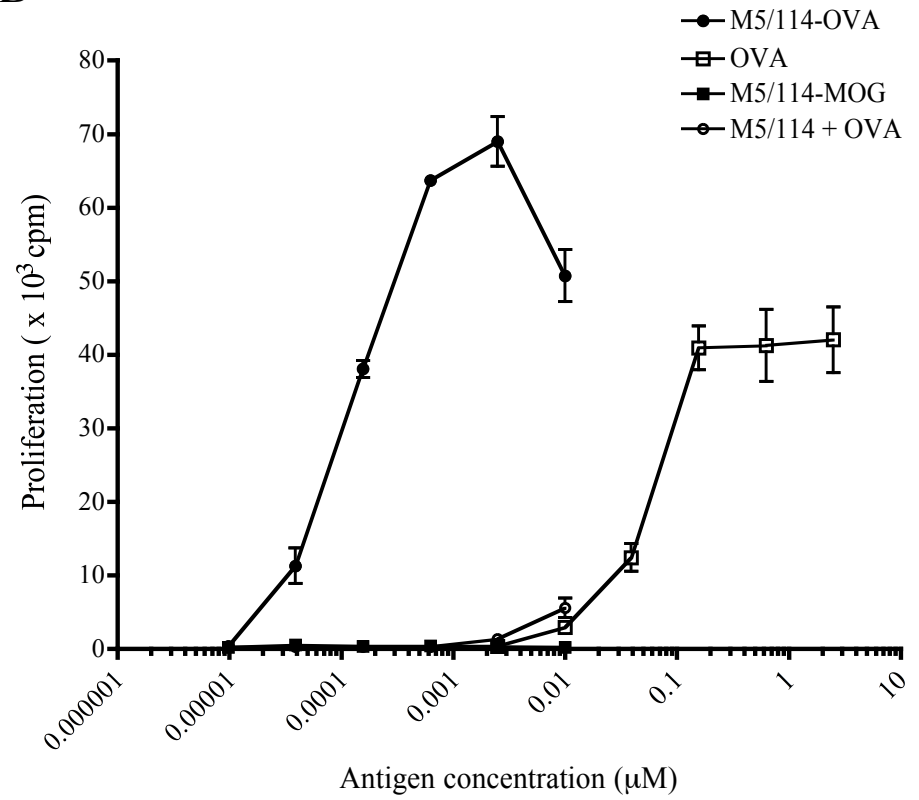

Figure 3.11: Targeting OVA to MHCII enhances CD4 T cell proliferation in vitro. (A) OVA protein was conjugated to M5/114. M5/114-OVA could not be separated from OVA. The purified (+) and unpurified (-) conjugates as well as loading controls were analysed by non-reducing SDS-PAGE and stained with Coomassie blue. MWM: Molecular weight marker. (B) Proliferation assay with splenocytes from OT-II mice. Splenocytes were incubated with the indicated concentration of antigens at $5 \times 10^{5}$ cells per well. The cells were incubated for 72 hours, and eight more hours in presence of $\left[{ }^{3} \mathrm{H}\right]$-thymidine. Proliferation was measured by the incorporation of $\left[{ }^{3} \mathrm{H}\right]$-thymidine. Data represents one out of three independent experiments (mean \pm SEM of triplicate wells). 


\subsubsection{Enhanced CD4 T Cell Proliferation is Independent of FcyR Mediated Uptake of M5/114-Antigen Conjugates}

$\mathrm{Fc} \gamma$ receptors $(\mathrm{Fc} \gamma \mathrm{R})$ are expressed by a variety of different cell types, including DCs, monocytes, macrophages and B cells (109). These receptors can bind to the constant Fc region of antibodies of the IgG isotype, and mediate uptake of the antibody and eventually bound or opsonised antigen into the cell interior (109). M5/114 is of the IgG2b isotype, and it was therefore possible that uptake of the M5/114 conjugates was mediated by Fc $\gamma$ Rs.

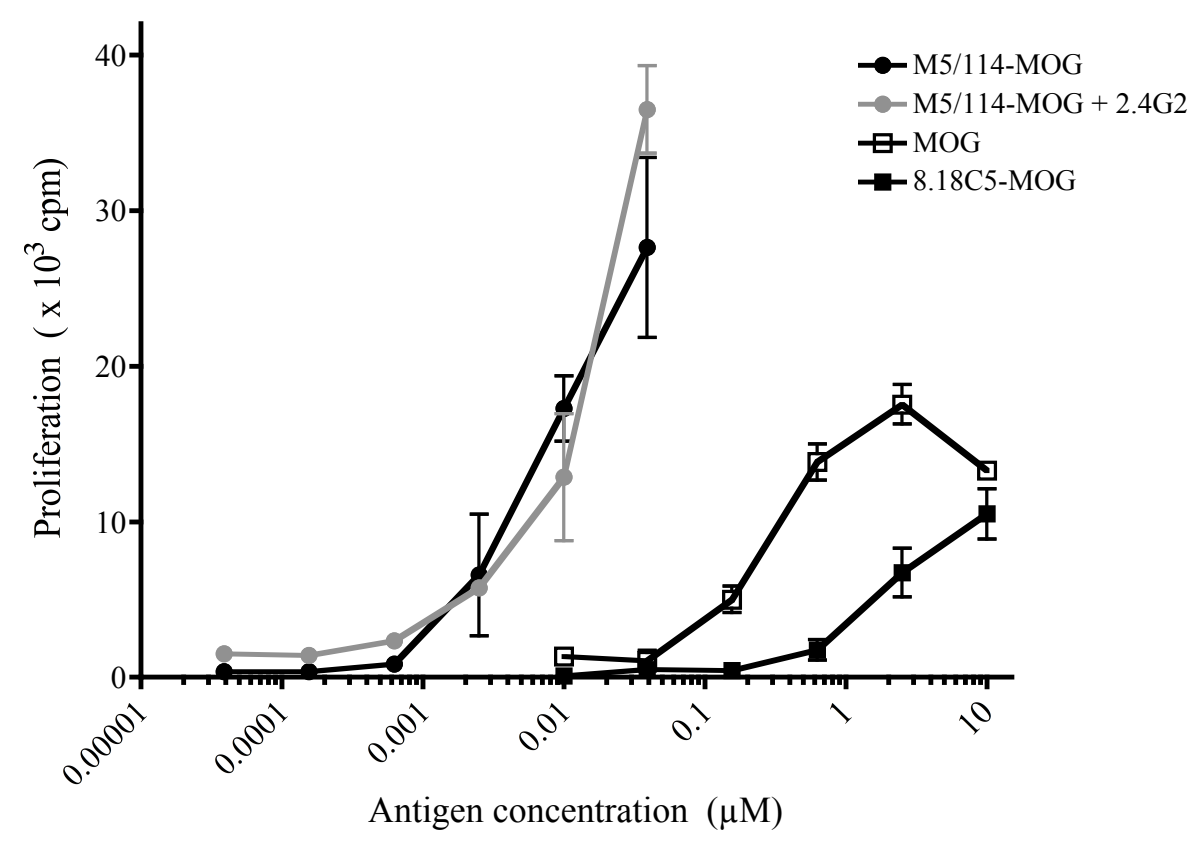

Figure 3.12: Increased CD4 $\mathrm{T}$ cell proliferation to M5/114-MOG is not diminished by Fc $\gamma$ RII/III blocking.

Splenocytes from 2D2 mice were incubated with M5/114-MOG at the indicated concentrations either with or without $1 \mu \mathrm{g} / \mathrm{ml}$ anti-CD16/CD32 antibody (clone 2.4G2) at $5 \times 10^{5}$ cells per well. MOG was used as a control antigen at the indicated concentrations. Anti-MOG-MOG (8.18C5-MOG) was used as a further control. The cells were incubated for 72 hours, and eight more hours in presence of $\left[{ }^{3} \mathrm{H}\right]$-thymidine. Proliferation was measured by the incorporation of $\left[{ }^{3} \mathrm{H}\right]$-thymidine. Data represents one out of two independent experiments (mean \pm SEM of triplicate wells).

To test whether Fc $\gamma$ Rs play a role in the uptake of M5/114-antigen conjugates, a proliferation assay was performed with M5/114-MOG in the presence of a blocking antibody specific for Fc $\gamma$ RIII/II (CD16/CD32, clone 2.4G2). The presence of 2.4G2 did 
not diminish M5/114-MOG induced CD4 T cell proliferation (figure 3.12). In addition, the use of an anti-MOG antibody (anti-MOG-MOG, clone 8-18C5) instead of M5/114 failed to induce $\mathrm{T}$ cell proliferation (figure 3.12). These results indicated that increased $\mathrm{T}$ cell proliferation in response to M5/114-MOG was most likely not due to increased uptake of antigens via $\mathrm{Fc} \gamma \mathrm{R}$ mediated pathways.

\subsubsection{Targeting OVA to MHCII enhances CD8 T cell Proliferation in vitro}

The enhanced CD4 T cell proliferation in response to MHCII targeted antigen was probably a result of increased antigen uptake and processing in endosomal compartments. As antigens can gain access to the MHCI pathway from early endosomes (98), the ability of the M5/114-antigen conjugates to increase antigen presentation on MHCI molecules was also investigated. For this, proliferation assays were performed using splenocytes from OT-I mice, which express a transgenic TCR on CD8 T cells that is specific for the $\mathrm{OVA}_{257-264}$ peptide. M5/114-OVA was 100-fold more potent in stimulating CD8 T cells than free OVA, whereas a mixture of unconjugated M5/114 and OVA failed to induce proliferation (figure 3.13).

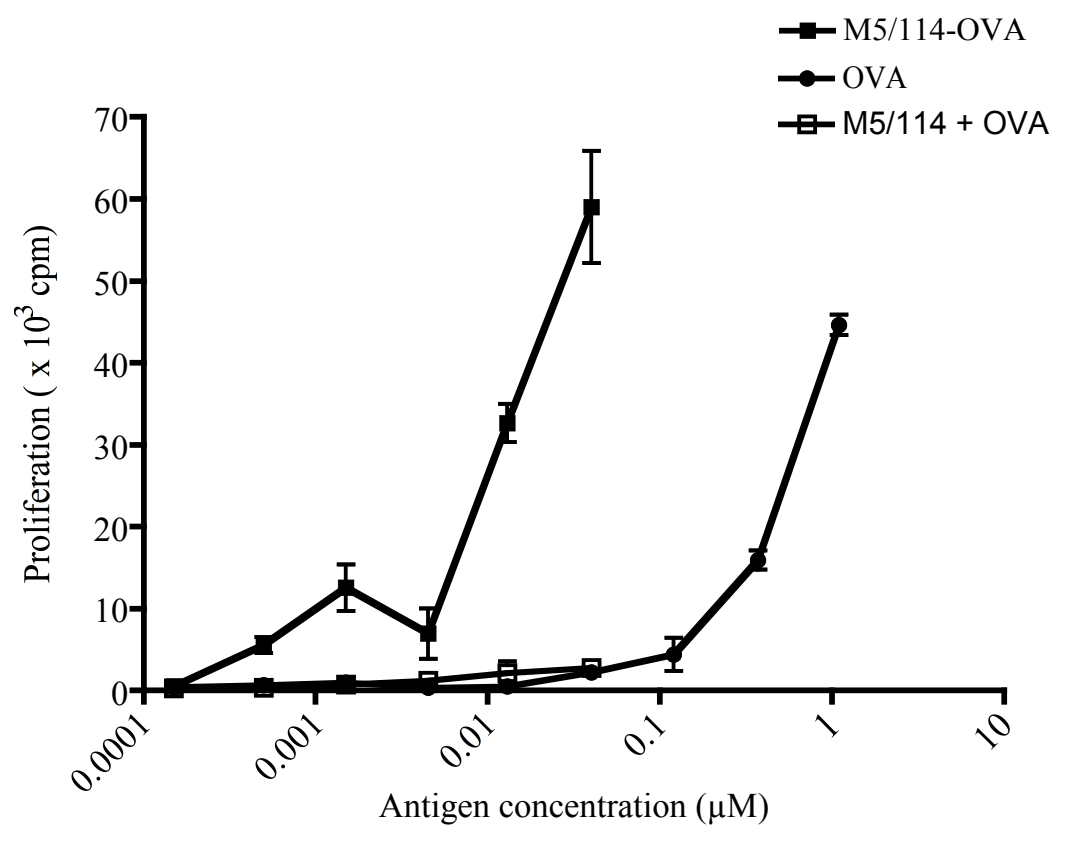

Figure 3.13: Targeting OVA to MHCII enhances CD8 $\mathrm{T}$ cell proliferation in vitro. Splenocytes from OT-I mice were incubated with the indicated concentrations of antigens at $5 \times 10^{5}$ cells per well. The cells were incubated for 48 hours, and eight more hours in presence of $\left[{ }^{3} \mathrm{H}\right]$-thymidine. Proliferation was measured by the incorporation of $\left[{ }^{3} \mathrm{H}\right]$-thymidine. Data represents one out of two independent experiments (mean $\pm \mathrm{SEM}$ of triplicate wells). 
Taken together, these findings suggested that conjugation of antigens to an antibody specific for MHCII was a suitable approach to deliver these antigens selectively to MHCII-expressing professional APCs. The improved antigen delivery to APCs resulted in a substantially enhanced induction of T cell proliferation by the APCs. Uptake of the conjugates was not mediated by $F c \gamma R$, and the conjugates were much more efficient in inducing proliferation of both CD4 and CD8 $\mathrm{T}$ cells than the respective free antigens.

\subsubsection{Antigen Targeting to MHCII with a Modified Superantigen in vitro.}

In order to further consolidate the effectiveness of targeting MHCII, MOG and OVA proteins were conjugated to an alternative targeting molecule, a modified SAg. The modified SAg SMEZ2 M1 has three point mutations that prevent its binding to TCRs, but MHCII binding is still intact (106). SMEZ2 M1 can therefore be used as a non-toxic vector for antigen-delivery to MHCII.

When MOG was conjugated to SMEZ2 M1 (SMEZ2 M1-MOG), it was a 100-fold stronger inducer of CD4 T cell proliferation than free MOG upon incubation with 2D2 splenocytes (figure 3.14A). Likewise, SMEZ2 M1-OVA induced proliferation at about 100-fold lower concentration than free OVA (figure 3.14B).

Contribution of free proteins that were present in the conjugation mixture could be excluded in these experiments, since free MOG or OVA proteins were essentially absent from the conjugates. As for the M5/114-antigen conjugates, T cell proliferation was antigen-specific, whereby SMEZ2 M1-MOG failed to induce proliferation of OT-II cells, and SMEZ2 M1-MOG-OVA did not induce proliferation of 2D2 cells.

These findings were consistent with the enhanced $\mathrm{T}$ cell proliferation induced by the M5/114 conjugates and emphasised the role of MHCII as a target for efficient delivery of antigen to APCs. 

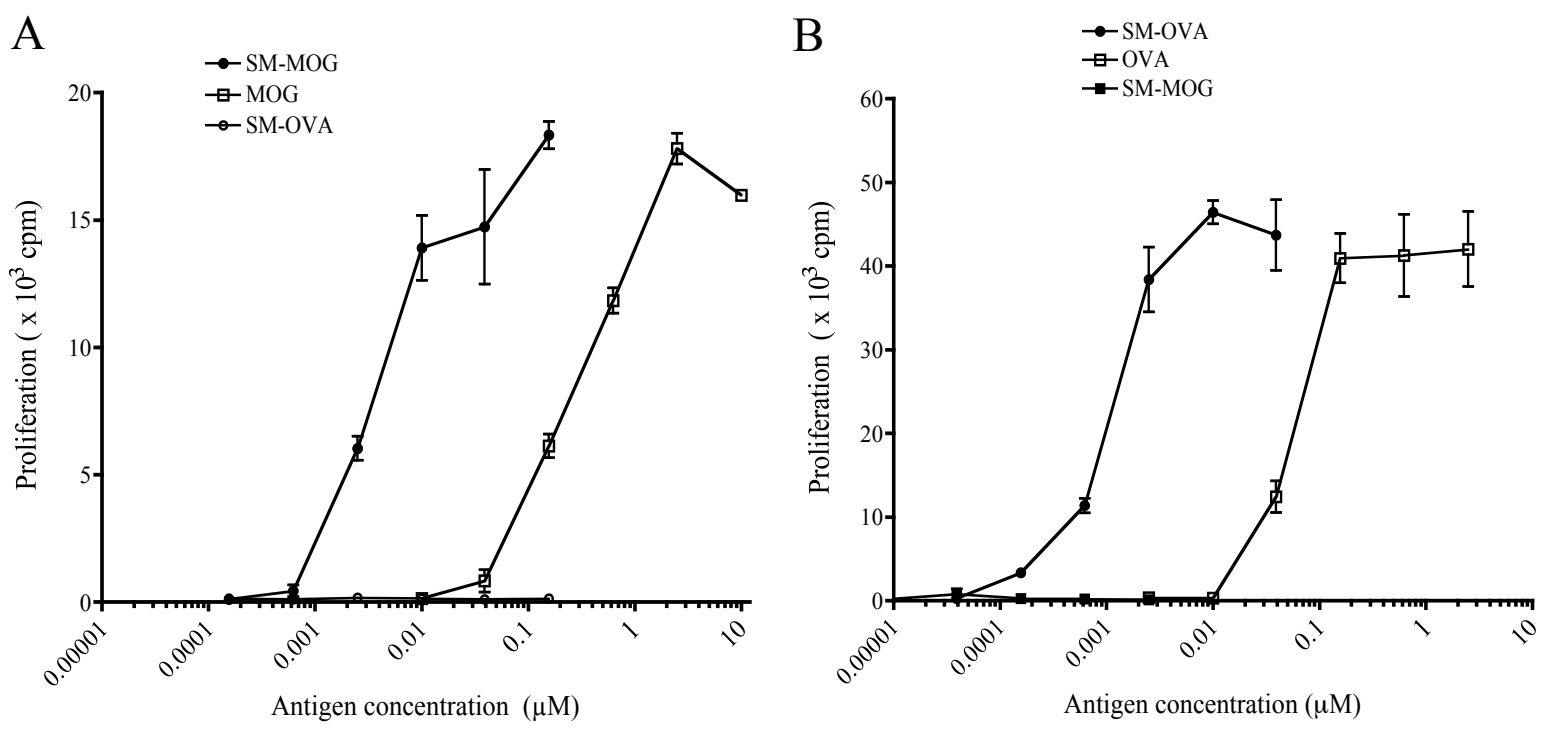

Figure 3.14: Targeting MOG and OVA proteins to MHCII with a modified SAg results in enhanced CD4 $\mathrm{T}$ cell proliferation in vitro.

The proliferative response to the SMEZ2 M1-MOG (A) or SMEZ2 M1-OVA (B) conjugates was measured using splenocytes from 2D2 (A) or OT-II (B) mice. Free MOG or OVA proteins and conjugates containing irrelevant antigen were used as experimental controls. Splenocytes were incubated with the indicated concentrations of antigens at $5 \times 10^{5}$ cells per well. The cells were incubated for 72 hours, and eight more hours in presence of $\left[{ }^{3} \mathrm{H}\right]$-thymidine. Proliferation was measured by the incorporation of $\left[{ }^{3} \mathrm{H}\right]$-thymidine. Data represents one out of three (A) or two (B) independent experiments (mean \pm SEM of triplicate wells).

\subsubsection{Targeting GA to MHCII Increases T Cell Proliferation in vitro}

The enhancement of T cell proliferation in the TCR transgenic models illustrated the potential for targeting GA to the MHCII pathway to enhance proliferation of GAspecific $\mathrm{T}$ cells to improve EAE suppression. To test the proliferative response to MHCII-targeted GA, proliferation assays were performed using draining lymph node (dLN) cells from mice previously immunised with GA.

GA was conjugated to M5/114 with the conjugation system that had been used for the conjugation of MOG and OVA to the same antibody (figure 3.15A). The most efficient conjugation was achieved at a ratio (GA:4FB) of 1:2, whereas the other conditions were the same as for the M5/114-MOG conjugation (see table 3.1). Free GA 
was removed by affinity chromatography (see table 3.2), and the final concentration of M5/114-GA was determined by $\mathrm{OD}_{280}$ measurement.

A

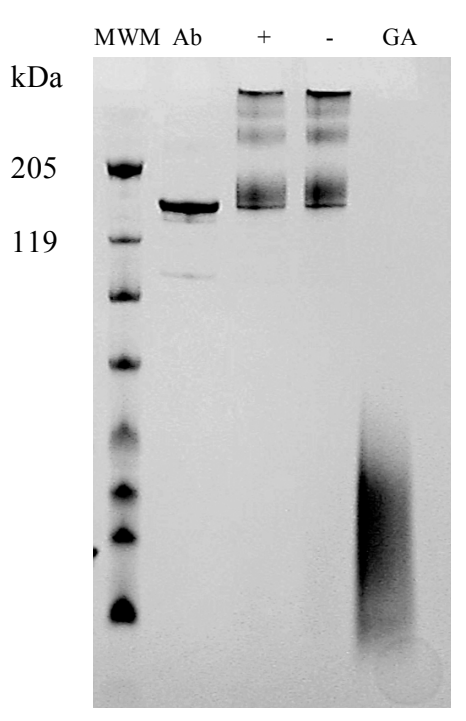

$\mathrm{C}$

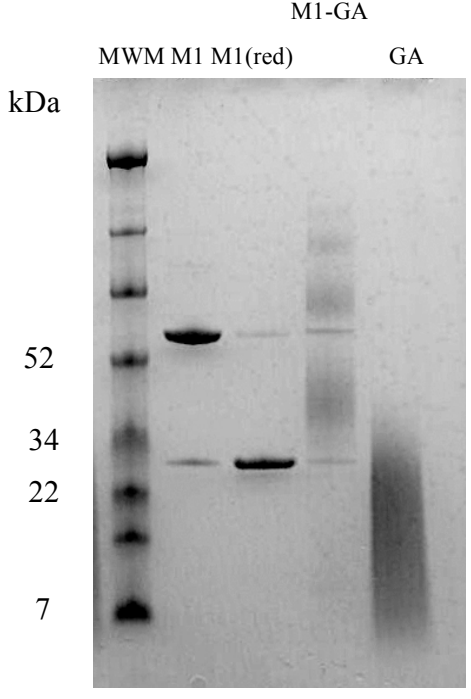

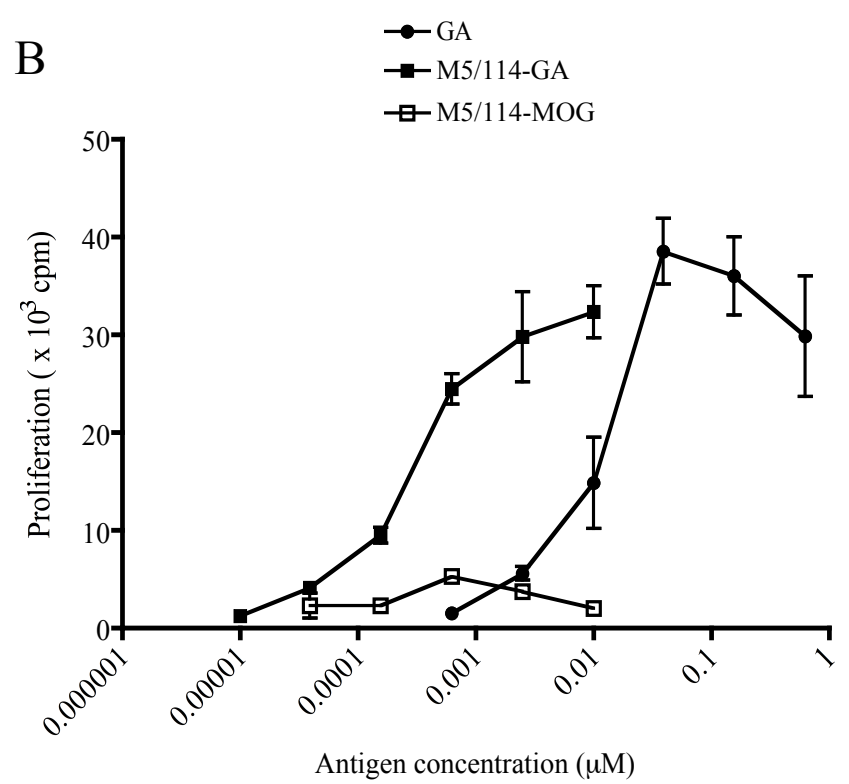

$\mathrm{D}$

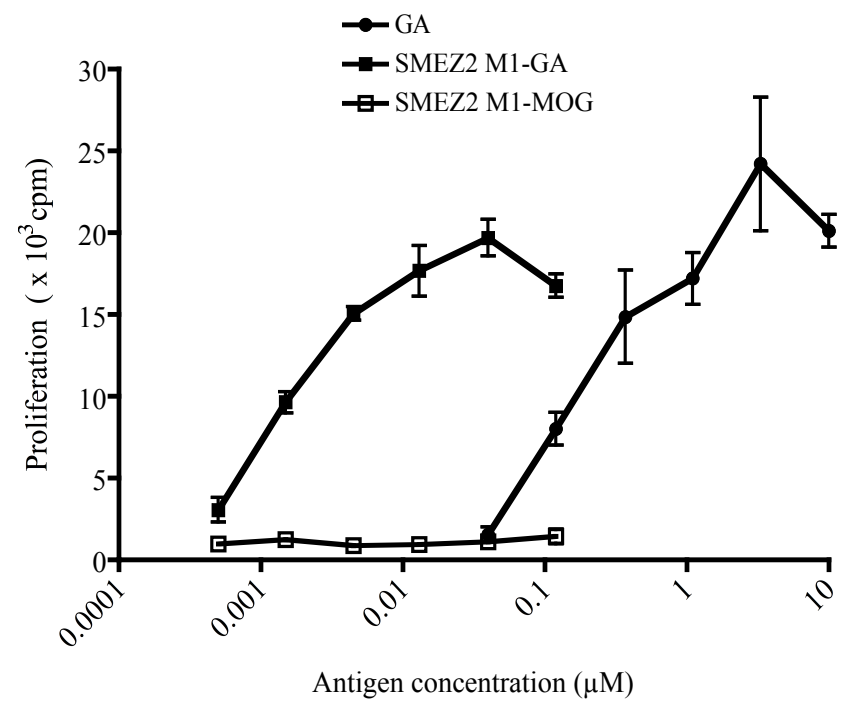

Figure 3.15: Targeting GA to MHCII results in enhanced T cell proliferation.

(A) GA was conjugated to M5/114 and purified by affinity chromatography. Purified (+) and unpurified (-) conjugates were analysed by non-reducing SDS-PAGE. M5/114 antibody $(\mathrm{Ab})$ and GA were loading controls. MWM: Molecular weight marker. (B) C57BL/6J mice were immunised with GA. Seven days after immunisation, draining lymph node cells were incubated with M5/114-GA, GA or M5/114-MOG for 80 hours, and 16 more hours in presence of $\left[{ }^{3} \mathrm{H}\right]$-thymidine. Proliferation was measured by the incorporation of $\left[{ }^{3} \mathrm{H}\right]$-thymidine. (C) GA was conjugated to SMEZ2 M1 as described in Materials and Methods. The conjugate was analysed by non-reducing SDS-PAGE and stained with Coomassie blue. Unconjugated oxidised and reduced SMEZ2 M1 (M1 and 
SMEZ2 M1 was conjugated to GA using SMCCplus, a heterobifunctional chemical, which has two functional groups that react with sulfhydryls and primary amines, respectively. First, GA was activated with SMCCplus, and then activated GA was co-incubated with reduced SMEZ2 M1 for conjugation. The reaction conditions were optimised (see A1.2 and A1.3), and the optimal conditions were a ratio (GA:SMCCplus) of 1:5; a ratio (SMEZ2 M1:GA) of 1:10, and an overnight incubation of activated GA with SMEZ2 M1 at $4^{\circ} \mathrm{C}$ (figure 3.15C). The conjugate contained only insignificant amounts of free GA, and was used in proliferation assays without need for further purification.

To test the potency of the GA conjugates to induce $\mathrm{T}$ cell proliferation, C57BL/6J mice were immunised with $500 \mu \mathrm{g}$ GA in CFA. Seven days later, cells were isolated from draining (inguinal) lymph nodes of the mice. The cells were incubated with GA or M5/114-GA and proliferation was measured. As shown in figure 3.15B, M5/114-GA was 100 -fold more potent inducer of $\mathrm{T}$ cell proliferation than free GA. Proliferation was also observed to M5/114-MOG, albeit at a much lower level. SMEZ2 M1-GA also induced proliferation of draining lymph node cells of GA-primed C57BL/6J mice at a 100-fold lower concentration than free GA (figure 3.15D).

Taken together, these results showed that antigens could be targeted to MHCII by conjugation to two different targeting molecules. This targeting resulted in markedly enhanced proliferation of TCR transgenic cells _, and also of antigen-primed nontransgenic $\mathrm{T}$ cells. This increased proliferation was therefore predicted to increase the efficacy of GA in EAE treatment, by promoting the expansion of protective $\mathrm{T}$ cells.

M1(red), respectively) and GA were controls. (D) C57BL/6J mice were immunised with $500 \mu \mathrm{g}$ GA. Seven days after immunisation, draining lymph node cells were incubated with SMEZ2 M1-GA, GA or SMEZ2 M1-MOG for 96 hours, and 16 more hours in presence of $\left[{ }^{3} \mathrm{H}\right]$-thymidine. Proliferation was measured by the incorporation of $\left[{ }^{3} \mathrm{H}\right]$-thymidine. Data (B and D) represents one out of two independent experiments each (mean \pm SEM of triplicate wells). 


\subsubsection{Targeting GA to MHCII does not suppress EAE}

Induction of GA-specific $\mathrm{T}$ cell responses is considered important for efficient protection from EAE (see figure 3.8 and ref. (62)). As a result, targeting GA to the MHCII antigen presentation pathway had the potential to significantly enhance $\mathrm{T}$ cell proliferation.

To test the potential of targeting GA to MHCII to improve EAE suppression, C57BL/6J mice were treated by co-immunisation with M5/114-GA. As shown in figure 3.16, $20 \mu \mathrm{g}$ M5/114-GA failed to ameliorate EAE. GA effectively suppressed EAE at a dose of $500 \mu \mathrm{g}$, but failed to suppress EAE at an equimolar amount to M5/114-GA (GA(e.m), figure 3.16).

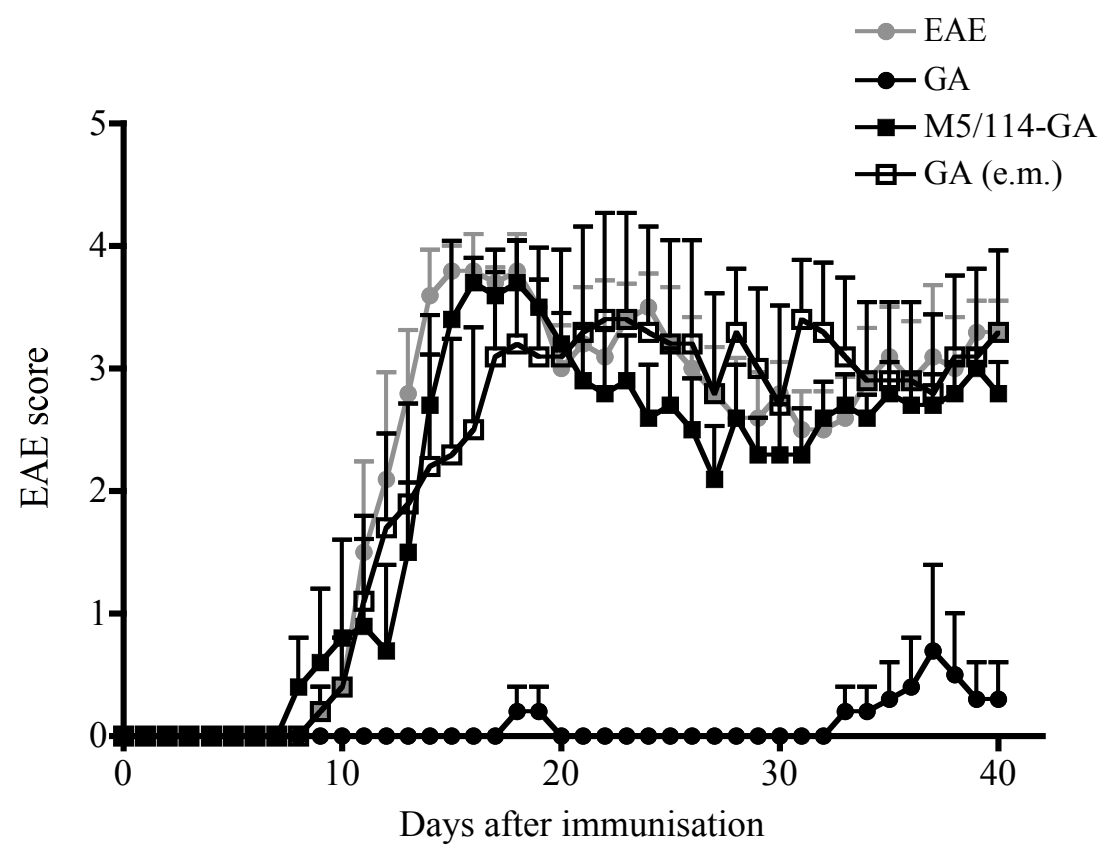

Figure 3.16: Targeting GA to MHCII with a monoclonal antibody does not suppress EAE.

C57BL/6J mice were left untreated (EAE), treated with $20 \mu \mathrm{g}$ M5/114-GA, or with $1.2 \mu \mathrm{g}$ GA (GA(e.m)) following the co-immunisation protocol. Mice were co-immunised with $500 \mu \mathrm{g}$ GA as a positive control. ( $\mathrm{p}>0.05$ for EAE vs. M5/114-GA and for EAE vs. GA (e.m), p $<0.001$ for GA vs. GA (e.m) and for GA vs. M5/114-GA; mean \pm SEM of $\mathrm{n}=5$ mice per group). Data represents one out of two independent experiments.

Similar results were obtained using the other MHCII targeting conjugate, SMEZ2 M1-GA (figure 3.17). This conjugate did not ameliorate EAE after coimmunisation with $5 \mu \mathrm{g}$ per mouse. M5/114-GA (estimated average MW $170 \mathrm{kDa}$, see 
figure 3.15A) and SMEZ2 M1-GA (average MW $40 \mathrm{kDa}$, see figure 3.15C) were used at comparable molar amounts in the EAE experiments.

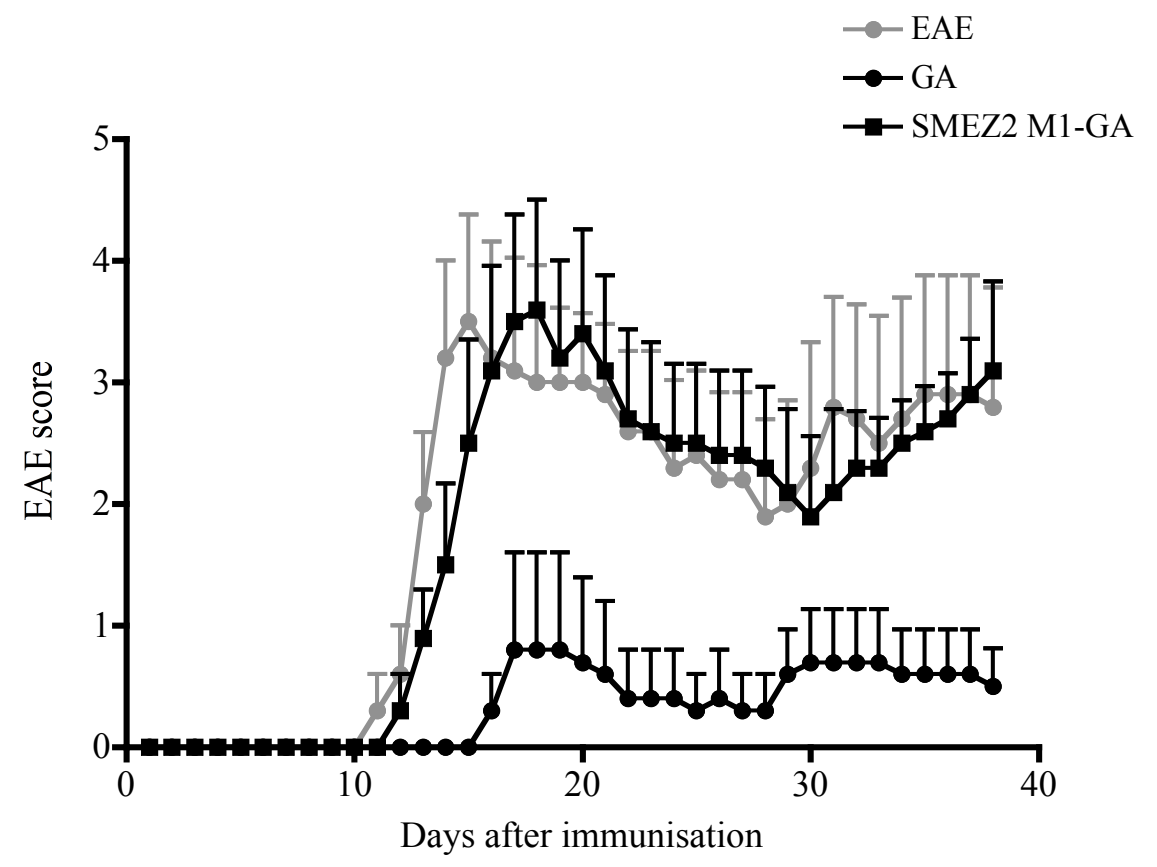

Figure 3.17: Targeting GA to MHCII with a modified SAg does not suppress EAE. C57BL/6J mice were left untreated (EAE), or co-immunised with $5 \mu \mathrm{g}$ SMEZ2 M1-GA, or with $500 \mu \mathrm{g}$ GA. ( $\mathrm{p}>0.05$ for EAE vs. SMEZ2 M1-GA, $\mathrm{p}<0.001$ for SMEZ2 M1-GA vs. GA; mean \pm SEM of $n=5$ mice per group). Data represents one out of two independent experiments.

\subsubsection{Summary}

A GA treatment model of EAE suppression was established to be used in investigating drug targeting strategies. In this model, co-immunisation of mice with $500 \mu \mathrm{g}$ GA resulted in efficient EAE suppression. This treatment mode was used as a control to evaluate the efficacy of MHCII targeted GA as a therapeutic agent.

Targeting antigens to the MHCII antigen presentation pathway enhanced CD4 and CD8 $\mathrm{T}$ cell responses by 100 - to 100 -fold . This could be achieved by the conjugation of antigens to two different targeting molecules, which have in common the high affinity binding to MHCII. Conjugation of GA to both targeting molecules also resulted in increased proliferation of GA-primed lymphocytes. However, treatment of EAE with GA conjugated to either of the targeting vectors was not effective. 


\subsection{Discussion}

Presentation of GA on MHCII molecules may be a key process for GA therapy of EAE and MS (figure 3.8 and ref. (62)). For this reason, targeting GA directly into the MHCII antigen presentation pathway was anticipated to result in an increase in therapeutic effect in EAE and hence in a marked decrease of the therapeutic dose. The results presented in this chapter involved the establishment of a GA treatment model of EAE that was subsequently used to investigate the drug targeting approach. The targeting strategy was validated by antigen targeting experiments before proceeding to the in EAE model.

\subsubsection{Establishment of a GA Treatment Model in C57BL/6J Mice}

For MS treatment, GA is injected subcutaneously at a dose of $20 \mathrm{mg}$ per day (86). In mice, GA can suppress EAE induced with MBP (68), MOG (39), PLP (110) and MSCH (111). EAE suppression can be achieved with different GA formulations, including subcutaneous treatments in CFA, IFA and PBS $(73,80,107)$. EAE suppression can be achieved by a single GA injection in CFA or IFA before or concomitantly with disease induction $(73,107,111)$. In absence of adjuvants, continuous GA treatment before disease induction or after disease onset is required $(80,82)$. In addition to these subcutaneous treatment models, GA also ameliorates MBP-induced EAE upon oral treatment. However, the oral route of administration has not proven beneficial for MS treatment in clinical trials (112).

The EAE model used in the current study involves the subcutaneous injection of $\mathrm{MOG}_{35-55}$ peptide emulsified in CFA. Immunisation of mice with the peptide in CFA induces an immune attack that is primarily directed against MOG epitopes and results in demyelination and axonal damage $(39,107) . \mathrm{MOG}_{35-55}$ induced EAE in $\mathrm{H}-2^{\mathrm{b}}$ mice displays a chronic disease course, and is often accompanied by optic neuritis, which results from demyelination and damage to the optic nerve $(39,92)$.

The objective of the experiments presented in this chapter was the establishment of a treatment model of EAE using GA as the therapeutic agent. GA has previously not been used in our laboratory for EAE therapy. Moreover, subtle differences in experimental settings can have substantial influence on therapeutic outcome, as discussed below. It was therefore necessary to establish a treatment model, on which subsequent drug targeting approaches could be based. 
First, the efficacy of subcutaneous GA injections in PBS was tested, because treatment without adjuvants is more readily applicable to MS treatment. The lack of EAE suppression after pre-treatment with GA in PBS over a seven-day period preceding disease induction (see figure 3.2) contradicts findings from other groups (80). The different results may be due to different treatment sites. In this study, mice received GA injections in PBS at the scruff of the neck, whereas in the other study, mice were treated in the hind limb flanks (M. S. Weber, personal communication). The contradicting results indicate that the treatment site is an important factor in GA treatment of EAE. Consistent with this, treatment with GA in CFA did not significantly improve EAE when given subcutaneously in the scruff of the neck, as opposed to the co-immunisation treatment in the flanks (see figure 3.6).

The observation that GA treatment needs to take place at the same site as EAE induction suggests that the drug modulates cells in the immediate surroundings of the injection site, which can then prevent or suppress the initiation of pathogenic $\mathrm{T}$ cell responses in the draining lymph node. It should be noted that, even in studies where the therapeutic formulations do not contain adjuvants , the injections take place at the same site as EAE induction; hence CFA is present at the site of treatment $(80,82)$. Therefore, CFA components may influence cells that GA eventually acts on, even when the GA formulation does not contain CFA.

Co-immunisation of mice with a single emulsion of CFA that contained both the encephalitogenic MOG peptide and GA proved highly efficient in suppressing EAE. This GA treatment efficiently prevented immune cell trafficking into the spinal cord parenchyma, and the majority of the treated mice lacked clinical signs of EAE over the complete observation period. The lack of effect of mannitol on EAE treatment was also confirmed, making co-immunisation an effective positive control for this study.

\subsubsection{GA does not Protect TCR Transgenic Mice from EAE}

2D2 mice express a transgenic TCR on their CD4 T cells that is specific for the $\mathrm{MOG}_{35-55}$ peptide (92). These mice develop spontaneous optic neuritis and more severe EAE than their wild-type counterparts upon immunisation with $\mathrm{MOG}_{35-55}$ (92).

In contrast to EAE suppression in wild-type mice, GA failed to alter the disease course in 2D2 mice (figure 3.8). Begum-Haque . recently reported a slight but statistically significant reduction of EAE severity in GA-treated 2D2 mice (82). EAE of 2D2 mice was milder than that of 2D2 mice used in this study (mean maximum EAE 
score 3 vs. 5). It is possible that disease severity plays a role in GA treatment, where GA is more effective in suppressing moderate disease pathology in 2D2 mice. However, both the results of this study and the study of Begum-Haque show that GA is less effective in treatment of EAE in 2D2 mice than in their wild-type counterparts (see figure 3.8 and ref. (82)).

Two potential scenarios can explain the lack of protection in 2D2 mice. First, an overwhelming pathogenic $\mathrm{T}$ cell response that overcomes GA-induced protection may be induced in the TCR transgenic mice by immunisation with $\mathrm{MOG}_{35-55}$ (82). Second, the absence of GA-specific T cell responses in the transgenic mice may be the reason for the lack of suppression. GA does not cross-react with MOG epitopes on TCR level (80), hence a GA-specific $\mathrm{T}$ cell response (including $\mathrm{T}_{\mathrm{H}} 2$ cells or T-regs) is absent in 2D2 mice. Presentation of disease-unrelated peptides by GA-conditioned monocytes induces expansion of Foxp3 $3^{+}$T-regs (80), but it is unknown whether this can happen with the disease-promoting $\mathrm{MOG}_{35-55}$ peptide . Hence a GA-specific T cell response is likely required for efficient EAE suppression.

Either individual scenario or a combination of both is plausible. Consistent with the second, GA is known to act through multiple mechanisms. Although not mentioned by the authors, $T$ cell independent mechanisms such as apoptosis of $T_{H} 1$ or $T_{H} 17$ cells (113) or neuroprotective effects (114) may be responsible for the minor beneficial effects of GA on moderate EAE observed by Begum-Haque (82). A further possible explanation for the discrepancy is that continuous daily GA treatment (82) may be able to ameliorate disease where co-immunisation (present study) fails.

\subsubsection{Targeting Antigens to MHCII Enhances T Cell Proliferation in vitro}

TCR transgenic 2D2 or OT-II mice were used to evaluate antigen presentation and $\mathrm{CD} 4 \mathrm{~T}$ cell proliferation in response to $\mathrm{MOG}$ and OVA conjugates, respectively. Both M5/114-MOG and M5/114-OVA caused a pronounced increase of proliferation relative to the respective free proteins (see figures 3.10 and 3.11). These results are consistent with the findings of Lunde

(115), who showed a similar increase in $\mathrm{T}$ cell proliferation using genetically engineered anti-MHCII antibodies. These "Troy-bodies" contained T cell epitopes within the constant region of their heavy chains. Increased antigen presentation required internalisation and processing of the Troy-bodies (115). 
Despite the different means of antigen incorporation into the anti-MHCII antibody, it is highly likely that the M5/114-antigen conjugates used in this study function by the same mechanism as Troy-bodies in antigen targeting. Internalisation and processing of the M5/114 conjugates are likely to be key processes for efficient antigen presentation, because the proteins are covalently bound to the antibody. Hence the mechanism probably involves the uptake of the M5/114-antigen conjugates by APCs, their processing in acidic compartments within the endocytic pathway, and the subsequent loading of the released peptides onto recycling MCHII molecules within the MIIC compartment (see figure 3.1).

The increased CD4 $\mathrm{T}$ cell proliferation in response to M5/114-MOG was not affected by blocking FcR $\gamma$ III/II with a specific antibody (116). In addition, a complex of MOG and MOG-specific IgG (clone 8-18C5) did not induce T cell proliferation (see figure 3.12). To note, the isotypes of M5/114 and $8-18 \mathrm{C} 5$ are $\operatorname{IgG} 2 \mathrm{~b}$ and $\operatorname{IgG} 1$, respectively. MOG-specific antibodies of the IgG2b isotype were not available. Although these findings do not formally exclude contribution of Fc $\gamma$ Rs that are not blocked by $2.4 \mathrm{G} 2$, they strongly suggest that uptake of M5/114 conjugates occurs through an MHCII dependent mechanism. A similar increase of antigen presentation was observed when the targeting molecule M5/114 was replaced by SMEZ2 M1 (see figure 3.14). Increased CD4 $\mathrm{T}$ cell proliferation in response to SMEZ2 M1-antigen conjugates is in consistence with previous findings (117), and further supports an MHCII-dependent uptake of the M5/114-antigen conjugates.

Various attempts to separate M5/114-OVA from free OVA failed, because M5/114-OVA did not bind to Ig specific affinity chromatography columns, and gel filtration yielded insufficient separation due to poor resolution of the column. (see table 3.2). Hence, M5/114-OVA could not be purified (see figure 3.11A) and was used as a mixture of M5/114-OVA and free OVA in the proliferation assays. However, it is unlikely that contaminating free OVA contributed substantially to increased proliferation. OVA concentration was much lower in wells supplemented with M5/114-OVA than in control wells containing free OVA protein (see figure 3.11). In addition, a mixture of unconjugated M5/114 and OVA failed to improve antigen presentation over free OVA.

In addition to increased antigen presentation on MHC II molecules, M5/114-OVA was also efficient in directing OVA peptides into the cross-presentation pathway (see figure 3.13). This observation is consistent with previous findings reporting that, upon 
incubation with SMEZ2 M1-OVA, DCs can efficiently present OVA peptides on MHCI molecules (117). Efficient stimulation of CD8 T cells with low doses of M5/114-OVA has great potential for the development of improved vaccines, considering that crosspresentation of free antigens is biased towards presentation of high-abundance antigens (95).

M5/114-GA and SMEZ2 M1-GA induced increased proliferation of GAprimed non-transgenic lymphocytes (see figure 3.15). Unlike cultures from TCR transgenic mice, lymph node cell suspensions from wild-type mice contain both CD4 and CD8 $\mathrm{T}$ cells that can be potentially reactive to GA. Hence, increased proliferation of lymph node cells may in part be attributable to CD8 T cells. However, expansion of $\mathrm{T}_{\mathrm{H}}$ cells in response to GA is well-established $(68,69,73,80)$, and enhanced proliferation of CD4 T cells was evident in the TCR transgenic systems. Targeting GA to MHCII is therefore likely to primarily induce CD4 T cell proliferation.

In summary, targeting antigens to the MHCII antigen presentation pathway resulted in significantly enhanced antigen uptake and presentation and gave rise to increased antigen-specific proliferation of CD4 and CD8 T cells . Next, it was tested whether the increased $\mathrm{T}$ cell proliferation in response to MHCII targeted GA was beneficial in EAE treatment.

\subsubsection{Targeting GA to MHCII in EAE Treatment}

Targeting antigens to professional APCs has been explored for a long time as a strategy for the development of improved vaccines (99). The major advantage of targeted vaccines is the potential of priming immune responses at low doses of antigen. Targeting antigens to cell surface molecules of DCs has also been shown to induce antigen specific tolerance in EAE (99), illustrating potential therapeutic applications in the treatment of diseases such as MS.

It has been reported more than two decades ago that subcutaneous immunisation with an anti-MHCII conjugated antigen can induce secondary serological responses to a later challenge with the free antigen in mice, even when the conjugates are administered without adjuvant (118). These responses are depend on MHCII haplotype compatibility to the antibody used for targeting (119). In addition, oral or intraduodenal immunisation with anti-MHCII conjugated antigens efficiently targets intestinal epithelial cells, and the targeted antigens are superior to free antigens in inducing specific serum $\operatorname{IgG}$ and $\operatorname{IgA}$ (120). 
In addition to the magnitude of the response, targeting antigens by conjugating them to anti-MHCII antibodies can also induce isotype switching (121). Hence targeting antigens to MHCII is a promising strategy for the generation of efficient antibody responses. However, MHCII has not been used as a target molecule for the induction of suppressive of regulatory $\mathrm{T}$ cell responses.

Although targeting GA to MHCII by conjugating it to M5/114 or SMEZ2 M1 significantly enhanced $\mathrm{T}$ cell proliferation , both M5/114-GA and SMEZ2 M1-GA failed to suppress EAE. The conjugates were used at a molar dose approximately 500 -fold lower than the therapeutic dose of free GA. The dose of conjugates used for EAE treatment were based on the finding that MHCII targeted antigens can induce CD4 $\mathrm{T}$ cell proliferation at concentrations 100- to 1000-fold lower than free antigen (see figures 3.10 and 3.15). However, it is possible that a higher dose of M5/114-GA is required for therapeutic effect than is necessary to induce proliferation

SMEZ2 M1-MOG $35-55$ has been shown to ameliorate EAE at a $10^{5}$-fold lower molar dose than the free peptide as a result of antigen specific tolerance (122). However, antigen-specific suppression of $\mathrm{MOG}_{35-55}$ specific $\mathrm{T}$ cells is unlikely to contribute to the effect of GA, since GA does not cross-react with $\mathrm{MOG}_{35-55}$ on TCR level (80). It therefore remains possible that the dose of GA conjugates used for treatment in this study was too low for therapeutic benefit.

Another possible explanation for the failure of the GA conjugates to suppress EAE is that internalisation and processing leads to the destruction of TCR epitopes that are contained within GA. The drug may be required to bind to MHCII directly without internalisation in order to stimulate protective $\mathrm{T}$ cells or to block pathogenic $\mathrm{T}$ cell responses (88). However, this is unlikely to be the case as the proliferative response to M5/114-GA showed that GA was also effective in inducing $\mathrm{T}$ cell responses after being internalised and processed to peptides (see figure 3.15).

Despite increased uptake and presentation of the drug by APCs, the conjugates may have failed to induce differentiation of the target cells into anti-inflammatory or tolerogenic APCs. Used as a conjugate, GA may have acted as a mere antigen, devoid of its immunomodulatory properties that promote the induction of anti-inflammatory or regulatory $\mathrm{T}$ cell responses $(73,80,84,85)$. Therefore, although the conjugates are capable of increasing $\mathrm{T}$ cell expansion, the $\mathrm{T}$ cells may not express the phenotype that is required for EAE suppression. 


\subsubsection{Conclusions}

Targeting proteins to MHCII expressing cells enhanced antigen-specific proliferation of $\mathrm{T}$ cells , as hypothesised. However, increased $\mathrm{T}$ cell proliferation did not translate into protection from EAE. The lack of EAE suppression may have been due to insufficient amounts of the GA conjugates used for treatment. However, other explanations for the failure of the conjugates are also possible.

Free GA induces a phenotype shift of APCs through an unknown mechanism, rendering them able to induce anti-inflammatory/regulatory $\mathrm{T}$ cell responses (80). Inability of the conjugates to induce this phenotype shift is a possible explanation for the lack of therapeutic effect of MHCII-targeted GA. Proliferation of GA specific T cells was observed in response to MHCII-targeted GA , but may by itself not be sufficient for disease suppression in absence of APC derived qualitative signals that promote differentiation of $\mathrm{T}_{\mathrm{H}} 2$ cells or T-regs.

It is not known whether GA can act through all MHCII expressing cells, or preferentially targets a particular APC subset. Hence sequestration of the conjugates by MHCII expressing cells that are irrelevant to GA's mechanism may have been responsible for the lack of protection. Thus targeting GA to a specific APC subset, rather than to total MHCII-expressing cells, may be a promising approach for treatment of EAE. 


\section{CHAPTER 4:}

\section{TARGETING GLATIRAMER ACETATE TO MONOCYTES IN EAE TREATMENT}




\subsection{Introduction}

MS and EAE are considered to be primarily CD4 T cell mediated diseases (51). However, there is evidence that monocytes and macrophages also substantially contribute to disease progression. Macrophages play important roles in the destruction of CNS tissue, the production of pro-inflammatory cytokines, and as APCs in the CNS (25). Depletion of macrophages in the CNS (123) or blockade of monocyte trafficking into the CNS (124) reduces severity of EAE, indicating that these cells are key players in the effector phase of the disease and are required for the development of clinical EAE.

In addition to disease-promoting functions, monocytes and macrophages can also play protective roles during treatment. GA is effective in treatment of MS and EAE suppression, and can alter monocyte or macrophage phenotype leading to their production of anti-inflammatory cytokines such as IL-10 or TGF- $\beta(80,125)$. In addition, GA treated monocytes are able to induce regulatory $\mathrm{T}$ cell responses that suppress EAE (80). Hence modulation of monocytes by GA may be a central part of its mechanism in MS treatment.

\subsubsection{Monocytes in Innate and Adaptive Immunity}

Monocytes are mononuclear leukocytes of myeloid lineage. They develop in the bone marrow from myeloid progenitors and are released into the bloodstream (126). They either reside in the blood as circulating monocytes, or enter peripheral tissue both under steady-state conditions and in response to inflammation, where they differentiate into macrophages or DCs (3). Monocytes fulfil important functions in innate immunity, including phagocytosis and clearance of pathogens or debris (127). In addition, monocytes can also induce adaptive immune responses by presenting antigen to $\mathrm{T}$ cells (128).

\subsubsection{Monocyte Subpopulations}

Monocytes are primarily identified by the expression of CD11b ( $\alpha_{M}$ integrin subunit) and low levels of F4/80 in mice (3). Recent findings have shown that murine monocytes can be further divided into at least two subpopulations that differ in their ability to respond to environmental signals and their potential to differentiate (129). One population expresses intermediate levels of the $\mathrm{CX}_{3} \mathrm{C}$-chemokine receptor-1 $\left(\mathrm{CX}_{3} \mathrm{CR} 1\right)$ and high levels of Ly6C $\left(\mathrm{CX}_{3} \mathrm{CR} 1^{\text {int }} \mathrm{Ly} 6 \mathrm{C}^{\mathrm{hi}}\right.$ monocytes $)$, a marker of unknown function 
that is part of granulocyte antigen-1 (Gr-1). The other subset expresses high levels of $\mathrm{CX}_{3} \mathrm{CR} 1$ but only low levels of Ly6C $\left(\mathrm{CX}_{3} \mathrm{CR} 1^{\text {hi }} \mathrm{Ly}^{6} \mathrm{C}^{\mathrm{lo}}\right.$, ref.(129)).

$\mathrm{CX}_{3} \mathrm{CR} 1^{\text {int }}$ Ly6 $\mathrm{C}^{\text {hi }}$ monocytes express the chemokine receptor CCR2 and can be recruited to sites of inflammation by MCP-1, the ligand for CCR2. Consequently, $\mathrm{CX}_{3} \mathrm{CR} 1^{\text {int }}$ Ly6 $\mathrm{C}^{\text {hi }}$ monocytes are commonly referred to as inflammatory monocytes (129). Inflammatory monocytes can home to sites of infection and differentiate into F4/80 hi macrophages or CD11c ${ }^{\text {hi }}$ DCs (3). Macrophages are highly phagocytic and play an important role in the clearance of pathogens and the resolution of inflammation (3). In addition, both macrophages and DCs can contribute to pathogen clearance by producing cytotoxic molecules such as nitric oxide (NO) and inflammatory cytokines such as tumour necrosis factor- $\alpha$ (TNF- $\alpha$, refs. $(3,130))$. Following their activation in inflamed tissue, inflammatory monocytes and monocyte-derived DCs also acquire the ability to present antigen and prime CD4 $\mathrm{T}$ cell responses (126).

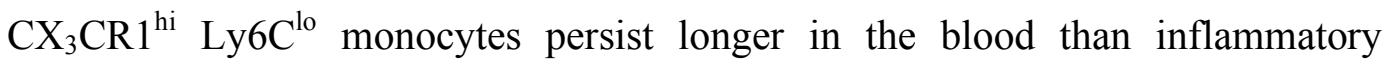
monocytes during homeostasis, and are therefore referred to as resident monocytes (129). The main function of resident monocytes is thought to be the replenishment of resident tissue macrophages, although they also have the potential to differentiate into DCs and to present antigen (126). Current data suggests that monocytes show the phenotype of inflammatory monocytes when they egress from the bone marrow and that differentiation into resident monocytes occurs subsequently to their release into the circulation (126, 131).

Monocytes gradually upregulate CD11c or F4/80 during their differentiation into DCs or macrophages, respectively (3). In most cases, CD $11 b^{\text {hi }} \mathrm{F} 4 / 80^{\text {lo }} \mathrm{CD} 11 \mathrm{c}^{-/ 1 \mathrm{lo}}$ monocytes can be distinguished from mature DCs $\left(\mathrm{CD} 11 \mathrm{c}^{\mathrm{hi}}\right)$ or macrophages $\left(\mathrm{F} 4 / 80^{\mathrm{hi}}\right.$, refs. $(3,131))$. However, phenotypical and functional traits of monocytes overlap with those of macrophages/DCs during differentiation, and classification of differentiating cells can be ambiguous (3). A more stringent definition refers to monocytes as mononuclear phagocytic cells in the circulation with the propensity to differentiate into macrophages, in addition to their $\mathrm{CD} 11 \mathrm{~b}^{\text {hi }} \mathrm{F} 4 / 80^{\text {lo }}$ cell surface phenotype (128). This definition is based on the assumption that differentiation programs are initiated in monocytes concurrently with their extravasation, and hence excludes cells that have left the bloodstream (128). 


\subsubsection{Differential Activation of Monocytes and Macrophages}

Inflammatory and resident monocytes play different roles in immunity. In addition, different types of stimuli can induce differential activation of monocytes and macrophages (3). Based on experiments of macrophage activation in response to defined stimuli, three common activation types have been identified to date: classical activation, alternative activation and type II activation (132).

Classical activation by inflammatory stimuli such as TLR ligands can trigger proinflammatory responses. Classically activated macrophages produce high levels of TNF- $\alpha$ and IL-12, have increased capacity to present antigen and produce high amounts of $\operatorname{ROS}(3,132)$. In contrast, alternative activation by anti-inflammatory cytokines (e.g. IL-4, IL-5) results in poor antigen presentation and the production of low levels of antiinflammatory cytokines (132). Type II activation can be achieved by immune complexes (e.g. Ig-opsonised antigen), and type II activated macrophages produce high levels of IL-10, but also show increased production of NO and efficient antigen presentation (132).

It is possible that monocytes respond to environmental stimuli in a similar fashion, and their differentiation into macrophages may happen concomitantly with differential activation $\quad$ For instance, GA treatment induces type II monocytes that produce high levels of IL-10 and efficiently present antigen to CD4 T cells However, classification of activation states is not as clear-cut in the more complex environment, and intermediate phenotypes of activation also exist $(3,131)$.

Production of NO is one feature of type II activated monocytes/macrophages, and high-level production of NO in peripheral lymphoid tissue is associated with $\mathrm{T}$ cell suppression in autoimmunity (45). Inflammatory monocytes with suppressive ability accumulate in spleens of mice immunised with CFA (133). These monocytes suppress T cell proliferation through production of NO (133). However, the role of these suppressive monocytes remains unclear, since mice develop clinical EAE despite their accumulation. 


\subsubsection{Myeloid-Derived Suppressor Cells}

Accumulation of cells expressing myeloid markers has been described in tumourbearing mice and in cancer patients (134). These cells have immunosuppressive ability, and are commonly referred to as myeloid-derived suppressor cells (MDSCs, ref. (135)). Myeloid cells with suppressive ability have also been found to accumulate in numerous other conditions such as bacterial and parasitic infections, acute and chronic inflammation, graft-versus host disease and autoimmune diseases (133, 134, 136-138). Although most of the studies have focused on the suppression of CD8 T cells that predominate in anti-tumour immunity, subsets of MDSCs have also been shown to suppress CD4 T cell responses (138).

Subsets of myeloid cells with suppressive ability include monocytes, macrophages, DCs, granulocytes and early myeloid progenitors (133, 139, 140). MDSCs can employ several mechanisms to suppress $\mathrm{T}$ cell proliferation in an antigen nonspecific manner, including IFN- $\gamma$ induced NO production (133), IL-4/IL-13 dependent arginase 1 (ARG1) upregulation (134) and inhibition of signalling through the TCR- $\zeta$ chain (136).

Subsets of MDSCs show augmented expression of the inducible NO synthase (iNOS) and suppress T cell responses through production of NO (139). NO-mediated suppression is associated with an inhibition of key intracellular signalling components such as Janus-activated kinases, STAT-3 or STAT-5, which results in $\mathrm{T}$ cell unresponsiveness, cell cycle arrest or apoptosis (139). NO may directly mediate these effects by oxidation or S-nitrosylation of the target proteins (134).

Some MDSC subsets upregulate ARG1 in response to $\mathrm{T}_{\mathrm{H}} 2$ cytokines like IL-4, IL-13, TGF- $\beta$ and IL-10, an enzyme that reduces L-arginine to urea and ornithine (139). ARG1 mediated suppression is associated with reduced expression of TCR- $\zeta$ chain on T cells, which renders T cells unresponsive to stimulation (139).

Subsets of MDSCs have different suppressive potential and employ different mechanisms to suppress immune responses $(139,140)$. In addition, individual MDSC subsets can exhibit suppressive function through more than one mechanism, as blockade of iNOS or ARG1 reduces, but does not completely abrogate suppression by MDSCs (140). Recent studies also suggest that, throughout a number of tumour and other disease models, mononuclear $\mathrm{CD} 11 \mathrm{~b}^{+} \mathrm{Gr}-1^{\text {lo }} \mathrm{F} 4 / 80^{\text {lo }}$ cells possess consistent and comprehensive 
suppressive ability, whereas suppressive potential of other MDSC subsets is more variable $(133,140,141)$.

\subsubsection{Objectives}

GA can induce the production of anti-inflammatory mediators by DCs, macrophages, monocytes and microglia $(84,85,125)$, and induce type II activation of monocytes (80). However, it is unclear whether GA preferentially targets a specific cell type or can act through multiple cell subsets . The primary aim of the experimental work presented in this chapter was to identify the target cell population of GA. The further objective was to characterise the target cells and to assess their potential to antigen non-specifically suppress $\mathrm{T}$ cell proliferation. Direct suppression of self-reactive cells is a potential mechanism that contributes to protection from EAE (45), and understanding the mechanisms of suppression could pave the way for the development of improved therapeutics.

Finally, targeting GA to its target cell population by conjugating it to a specific monoclonal antibody was hypothesised to enhance the therapeutic efficacy of GA in EAE and to substantially reduce the therapeutic dose. Drug targeting by the use of monoclonal antibodies is a promising approach for the treatment of many diseases (99), and GA targeting has the potential of clinical application in MS therapy. 


\subsection{Results}

\subsubsection{GA Binds to CD11b ${ }^{\text {hi }}$ Cells in vivo, but not in vitro}

GA can modulate $\mathrm{CD}_{11} \mathrm{~b}^{+} \mathrm{CD} 11 \mathrm{c}^{-}$monocytes

(80), but it is not clear whether this is a result of direct action on monocytes. The primary target cell population of GA is currently not known. GA has been shown to bind to MHCII expressing cells

(87). However, it is currently unclear whether or not

GA binding is specific to a particular type of MHCII expressing cell. In addition, targeting GA to MCHII is ineffective in suppressing EAE at low dose (see figures 3.16 and 3.17). It is therefore possible that MHCII molecules are not the main targets of GA.

To identify its target cell population, GA was labelled with Alexa Fluor $^{\circledR} 488$ (Alexa488), and injected intravenously to C57BL/6J mice. Cell suspensions were prepared from the blood, spleen and lymph nodes (inguinal, para-aortic or mesenteric) one hour after the injections, and analysed by flow cytometry (figure 4.1). GA bound to a population of cells in the blood and spleen that expressed high levels of $\mathrm{CD} 11 \mathrm{~b}\left(\mathrm{CD} 11 \mathrm{~b}{ }^{\mathrm{hi}}\right)$ and located to the mononuclear fraction on the side scatter $\left(\mathrm{SSC}^{\mathrm{lo}}\right)$. Alexa488 labelled OVA was used as control, and did not bind to the same cells. Bovine trypsinogen, an inactive precursor of the intestinal protease trypsin, and DM SMEZ2, a modified bacterial superantigen, also bound to $\mathrm{CD} 11 \mathrm{~b}{ }^{\text {hi }}$ cells in both blood and spleen (figure 4.1). MOG protein was included as a further control protein and, like OVA, did not bind to any cells.

The percentage of $\mathrm{GA}^{+}$cells showed considerable variation between individual mice and this variation correlated with the percentage of total $\mathrm{CD} 11 \mathrm{~b}^{\text {hi }} \mathrm{SSC}^{\text {lo }}$ cells (compare figures 4.1 and 4.2). Hence the variation was not due to differences in GA binding, but due to variance in the presence of target cells. In addition, GA, but not DM SMEZ2 or trypsinogen, also bound to a significant percentage of CD11 b cells in blood and spleen, but the fluorescence intensity was much lower than the staining of CD11b ${ }^{\text {hi }}$ cells (figure 4.1). 


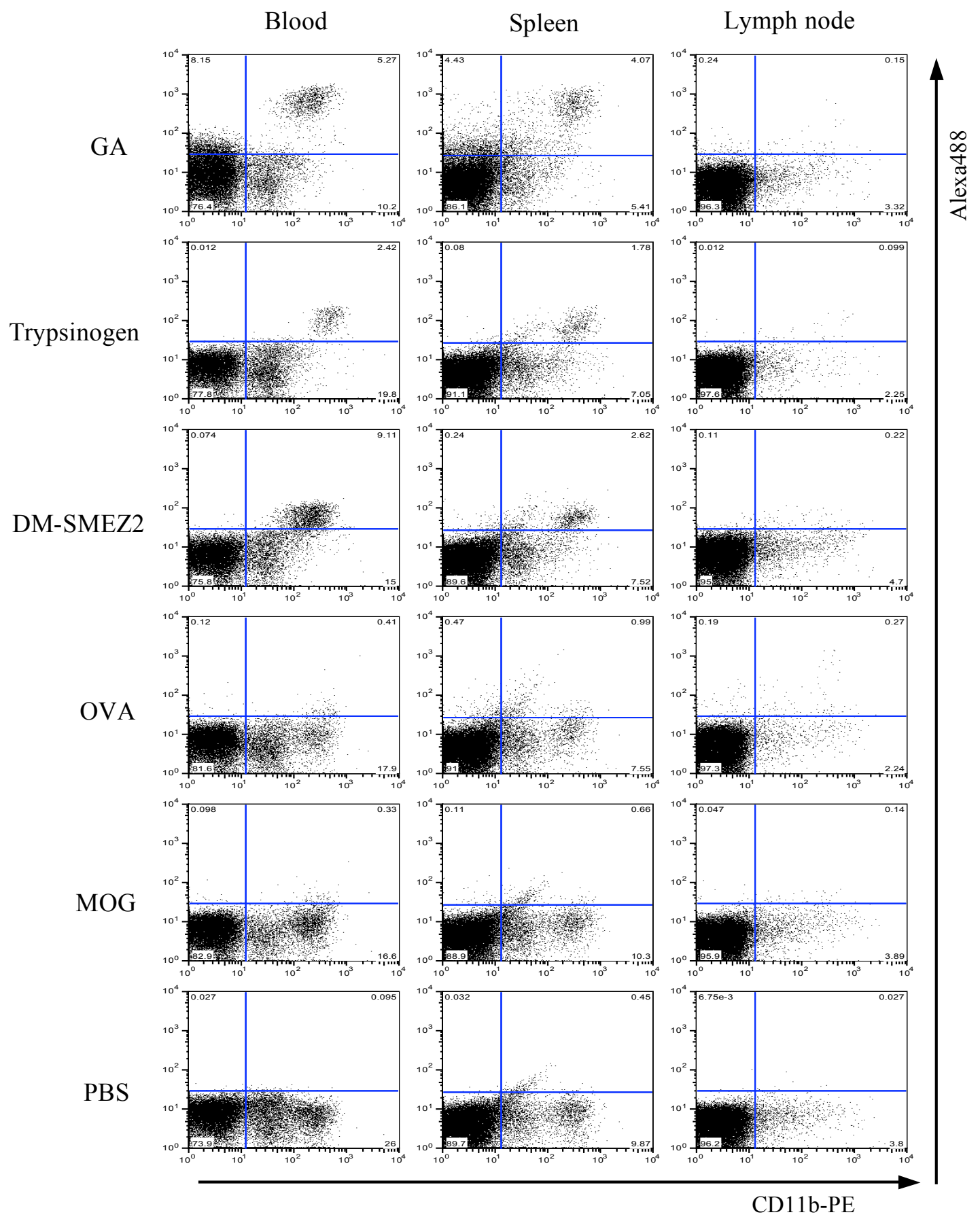

Figure 4.1: GA binds to a $\mathrm{CD}_{11 b^{\text {hi }}}$ cell population after intravenous injection.

C57BL6/J mice received intravenous injections of $50 \mu \mathrm{g}$ Alexa488 labelled proteins in PBS. Cells from indicated tissues were recovered one hour after the injections, stained for cell surface antigens and cells of individual mice were analysed by flow cytometry. Plots are gated on mononuclear cells. Data represents one out of at least three independent experiments for each protein. 
Binding to $\mathrm{CD} 11 \mathrm{~b}^{\text {hi }}$ cells was also observed when the mice were injected with fluorescein isothiocyanate (FITC) labelled GA (figure 4.2); hence GA staining was not limited to one particular fluorophor. However, GA staining could not be detected upon intravenous injection of biotinylated GA and subsequent incubation of blood cells with PerCP-conjugated streptavidin

(figure 4.2).
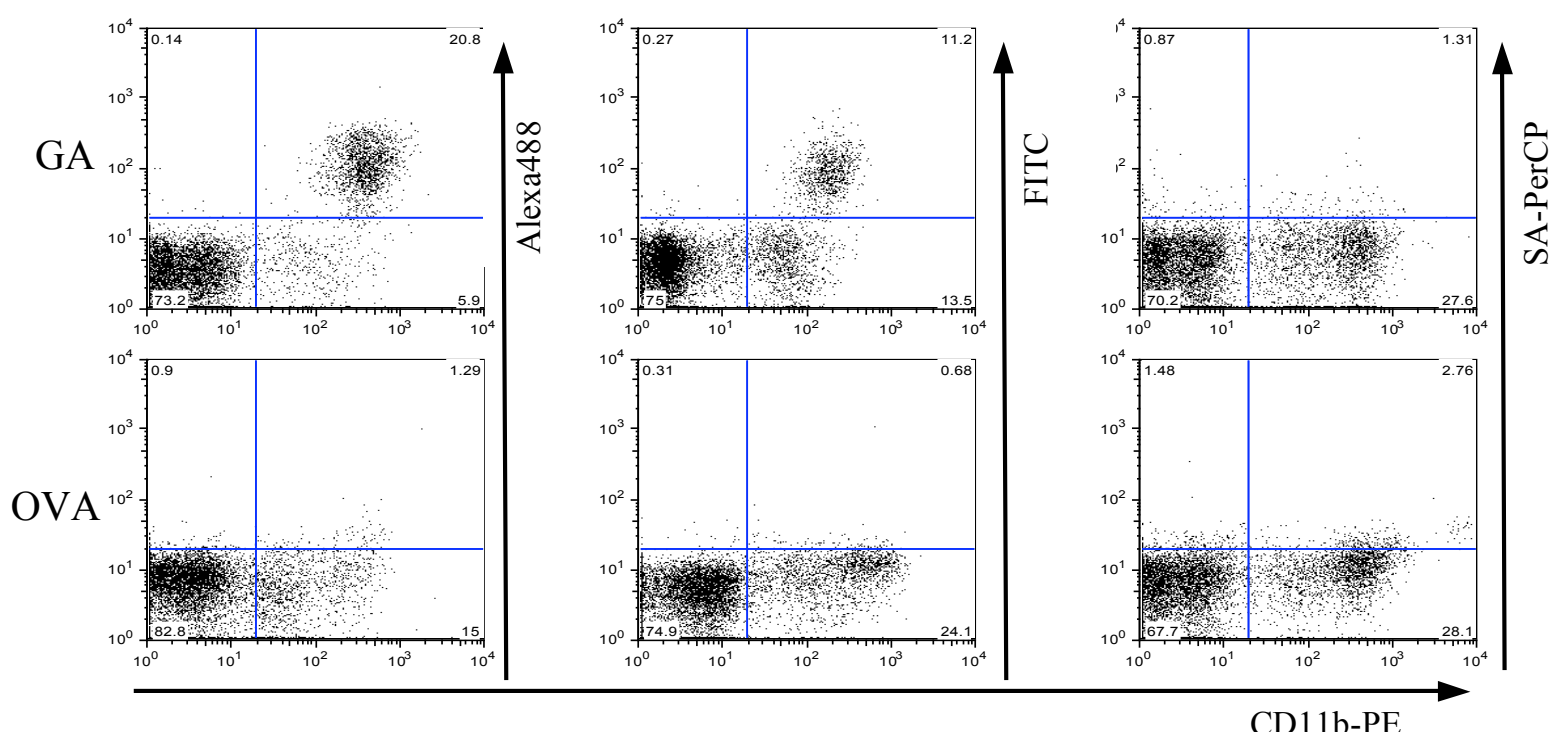

Figure 4.2: GA staining of $\mathrm{CD}_{11} \mathrm{~b}^{\text {hi }}$ cells with different fluorophors.

C57BL6/J mice received intravenous injections of $50 \mu \mathrm{g}$ of GA or OVA, which had been labelled with Alexa488, FITC or biotinylated. Cells from the blood were recovered one hour after the injections and stained for cell surface antigens. Cells from mice that had received biotinylated proteins were stained with streptavidin (SA)-PerCP. Cells from individual mice were analysed by flow cytometry. Plots are gated on mononuclear cells. Data represents one out of two independent experiments $(n=2$ mice per group).

To test whether binding to CD11 $\mathrm{b}^{\text {hi }}$ cells would occur , Alexa488 labelled GA (GA-Alexa488) was incubated with single cell suspensions from blood, spleens or lymph nodes of $\mathrm{C} 57 \mathrm{BL} / 6 \mathrm{~J}$ mice at $37^{\circ} \mathrm{C}$ in PBS containing $1 \% \mathrm{FCS}$ for one hour. The cells were then analysed by flow cytometry for GA staining. Even though some CD11b ${ }^{\text {hi }}$ cells showed weak GA staining compared to OVA (figure 4.3), the staining was much less pronounced than the staining of $\mathrm{CD} 11 \mathrm{~b}^{\text {hi }}$ cells by GA. Some CD11b cells showed distinct GA staining (figure 4.3), and these cells were heterogeneous in MHCII expression (see A1.4). The pattern of staining was essentially the same upon incubation in buffer supplemented with $0.1 \%$ sodium azide (data not shown). Hence, the 
weak GA staining

was likely due to binding to cell surface molecules, rather than fluid-phase pinocytosis, since OVA-Alexa488 stained less cells than GA and GA staining was also observed in azide-containing buffer (142).
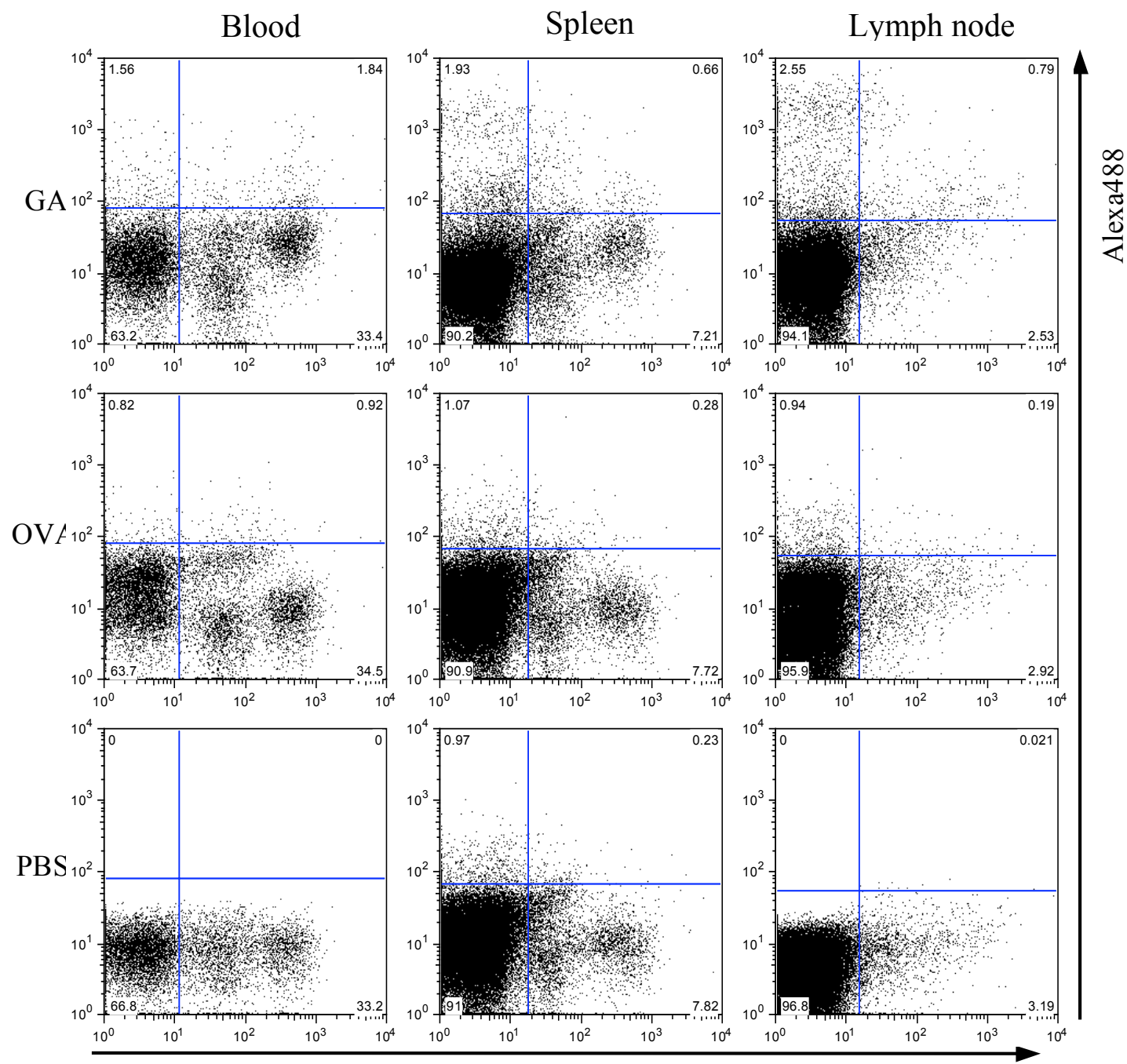

CD11b-PE

\section{Figure 4.3: GA does not bind to $\mathrm{CD}_{11} \mathrm{~b}^{\text {hi }}$ cells in vitro.}

Single cell suspensions were prepared from spleens, inguinal lymph nodes and blood of C57BL/6J mice. The cells were incubated in absence (PBS) or presence of $10 \mu \mathrm{g} / \mathrm{ml}$ Alexa488 labelled GA or OVA for one hour at $37{ }^{\circ} \mathrm{C}$ in a 96 -well plate at $5 \times 10^{4}$ cells per well. The cells were then stained for cell surface markers and analysed by flow cytometry. Data represents one out of two independent experiments with duplicate wells.

In summary, GA binding to CD11 $\mathrm{b}^{\text {hi }}$ cells was observed in blood and spleen using two different fluorophors, but significant staining of $\mathrm{CD}_{11} \mathrm{~b}^{+}$or $\mathrm{MHCII}^{+}$ cells was not observed upon incubation with cell suspensions from the same tissues. 


\subsubsection{GA Cells in the Blood Show Monocyte Cell Surface Phenotype}

Next, the cells that showed GA staining were further characterised based on their expression of cell surface markers. GA-Alexa488 was injected intravenously as above, and cell surface marker expression of GA-binding cells in the blood was examined. GA binding cells were heterogeneous in expression of Ly6C, Gr-1, CD11c and MHCII (figure 4.4).

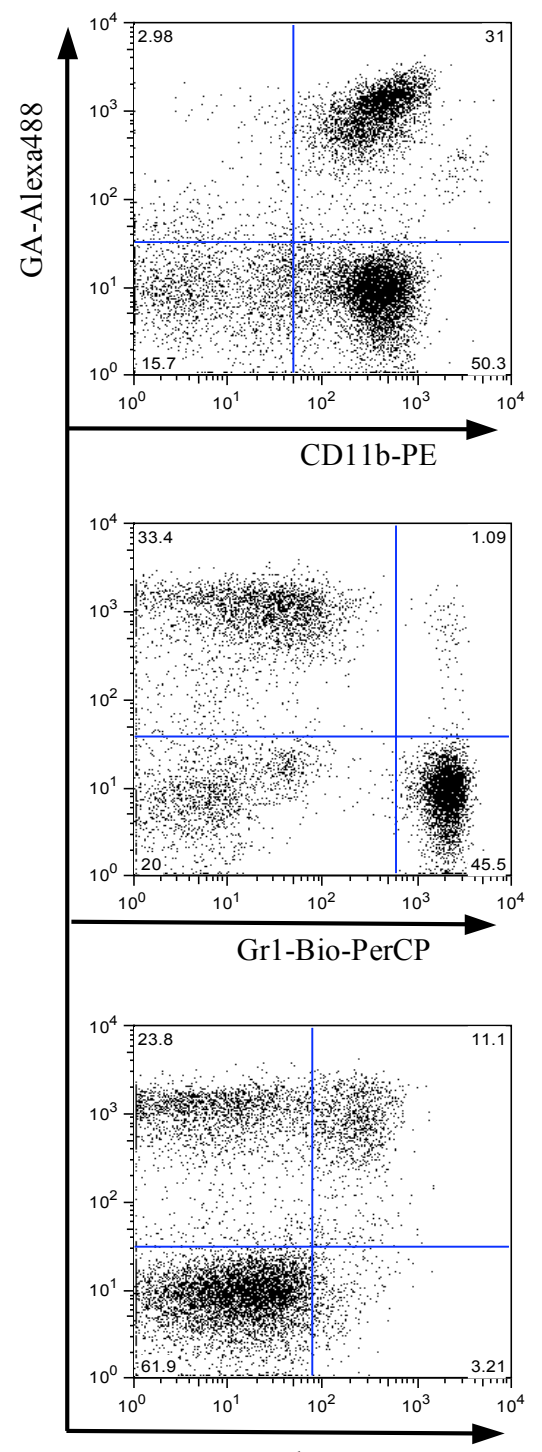

IA $^{\text {b-Alexa647 }}$
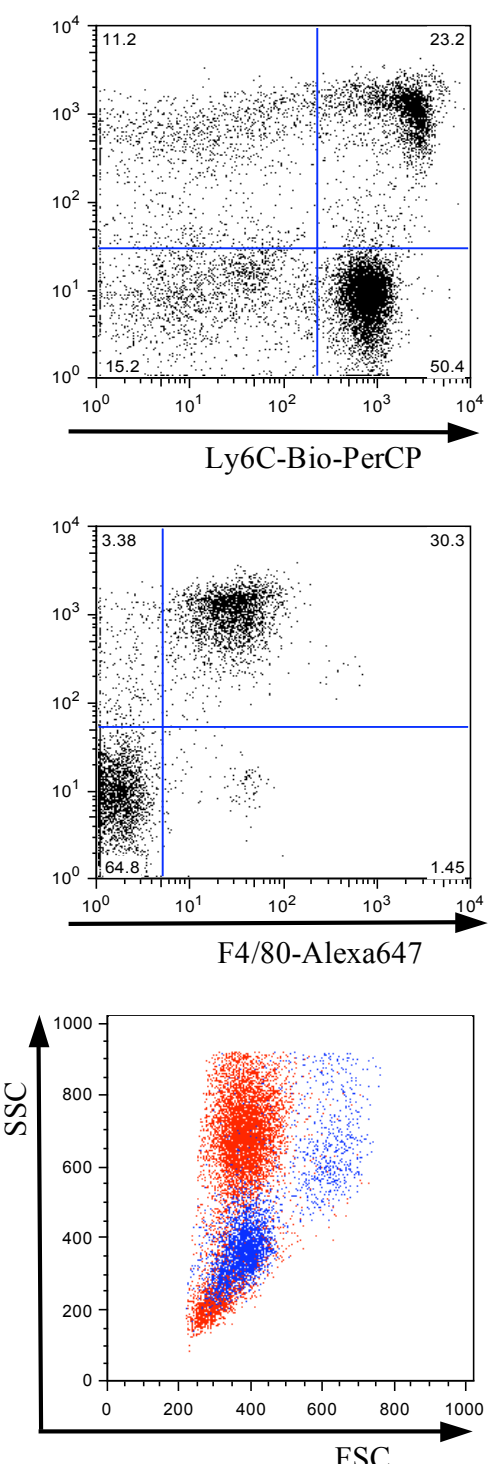
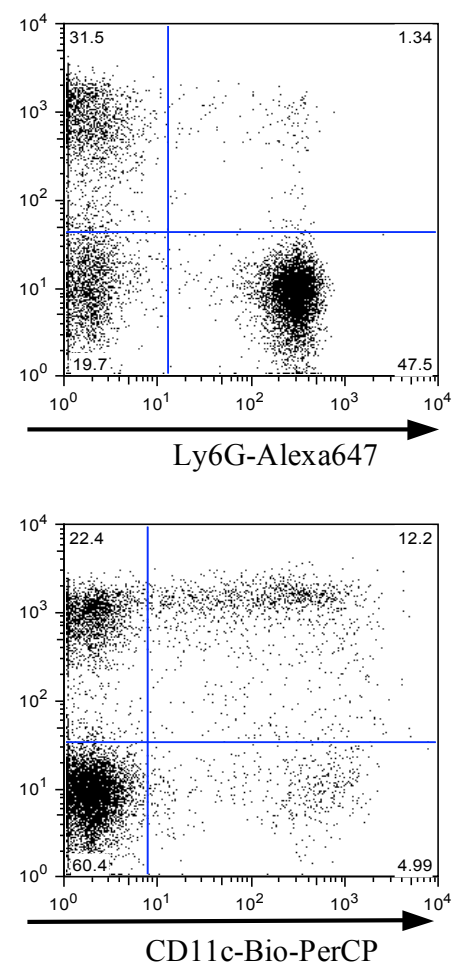

$\mathrm{GA}+$

total cells

Figure 4.4: $\mathrm{GA}^{+}$cells in the blood show monocyte cell surface phenotype.

GA-Alexa488 was injected intravenously to C57BL/6J mice at a dose of $50 \mu \mathrm{g}$ per mouse. CD11 bi cells were enriched from pooled blood of five mice by magnetic sorting one hour after the injections. The cells were then stained for cell surface markers and analysed by flow cytometry. Bottom right panel row shows the FSC/SSC profile of $\mathrm{GA}^{+}$ cells in comparison to total blood cells. Data represents one out of two experiments. 
Virtually all cells that bound GA lacked cell surface expression of Ly6G and were $\mathrm{SSC}^{\mathrm{lo}}$, indicating that the cells were not neutrophils or other granulocytes (140). GAbinding cells expressed low levels of F4/80 and high levels of CD11b (figure 4.4), indicating that they were monocytes (3). The heterogeneity in Ly6C and Gr-1 indicates that both inflammatory and resident monocytes were contained within the GA binding population (131).

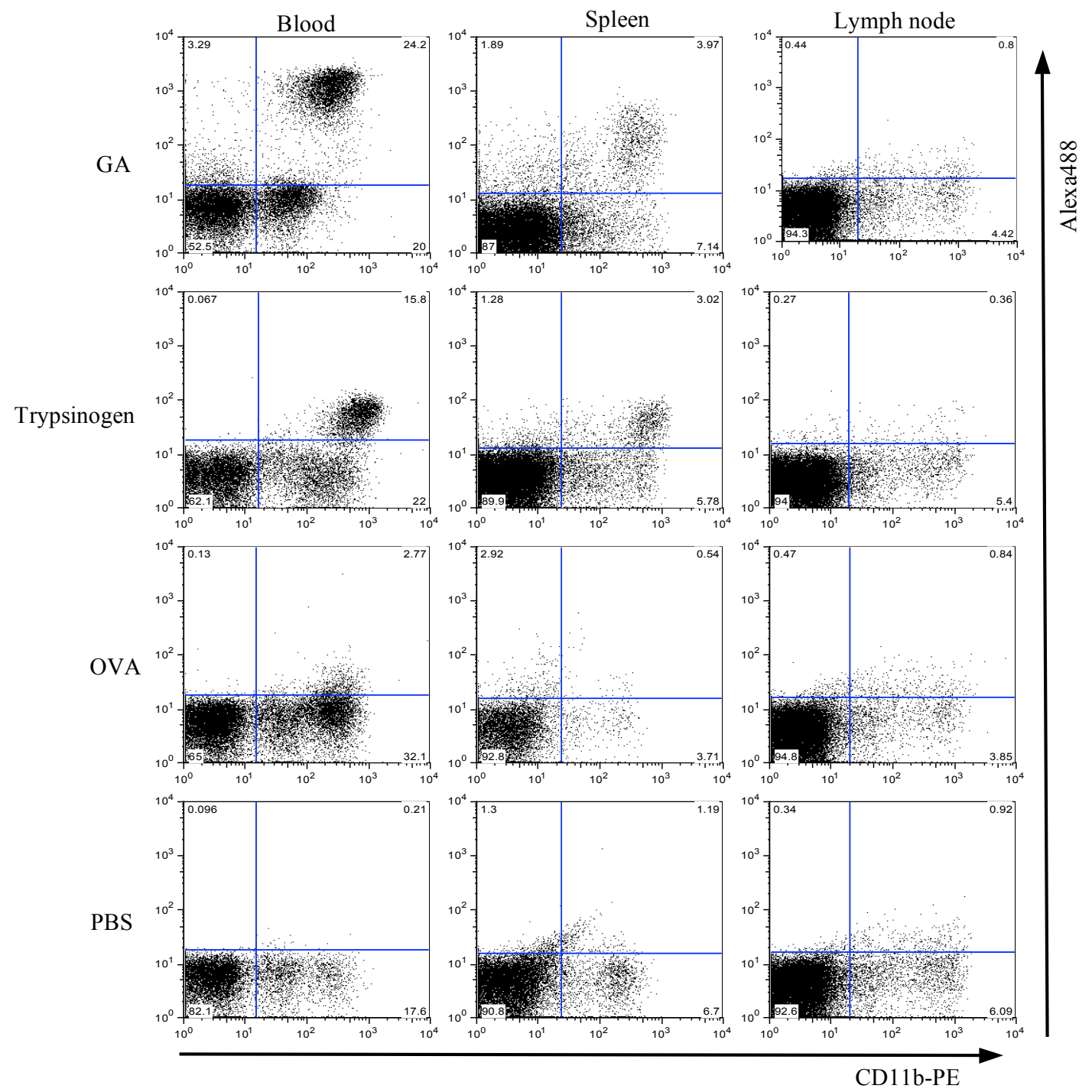

Figure 4.5: Binding of GA to CD11b ${ }^{\text {hi }}$ cells is independent of MHCII.

MHCII deficient $\mathrm{B} 6 \mathrm{Aa}^{0} / \mathrm{Aa}^{0}$ mice received intravenous injections of $50 \mu \mathrm{g}$ of Alexa488labelled proteins in PBS. Cells were recovered from the indicated tissues of individual mice one hour after the injections. The cells were stained for cell surface markers and analysed by flow cytometry. Plots are gated on mononuclear cells. Data represents one of two independent experiments. 
Both GA and trypsinogen bound to monocytes in MHCII deficient $\mathrm{B} \mathrm{Aa}^{0} / \mathrm{Aa}^{0}$ mice (figure 4.5). The percentage of cells that bound GA and trypsinogen were higher than in wild-type mice, due to an elevated overall percentage of monocytes in the blood of MHCII deficient mice. In addition, GA-binding cells in MHCII deficient mice were also $\mathrm{F} 4 / 80^{\mathrm{lo}}$ and $\mathrm{Ly}_{6 \mathrm{G}}^{-}$, similar to $\mathrm{GA}^{+}$cells in wild-type mice (see A1.5).

In summary, GA bound to a heterogeneous population of monocytes in the blood after intravenous injection. The binding was independent of MHCII, because virtually all CD11b ${ }^{\text {hi }}$ cells of the mononuclear fraction bound GA in both wild-type and MHCII deficient mice (see figures 4.1 and 4.5).

\subsubsection{Time-course of Monocyte Staining by $\boldsymbol{G A}$}

In the above experiments, blood cells were recovered for analysis one hour after injection of fluorophor-labelled GA. To investigate the time profile of GA staining, GA-Alexa488 was intravenously injected, and blood cells of individual mice were analysed over time. Consistent with previous experiments, more than $90 \%$ of monocytes were $\mathrm{GA}^{+}$one hour after injection (figure 4.6).
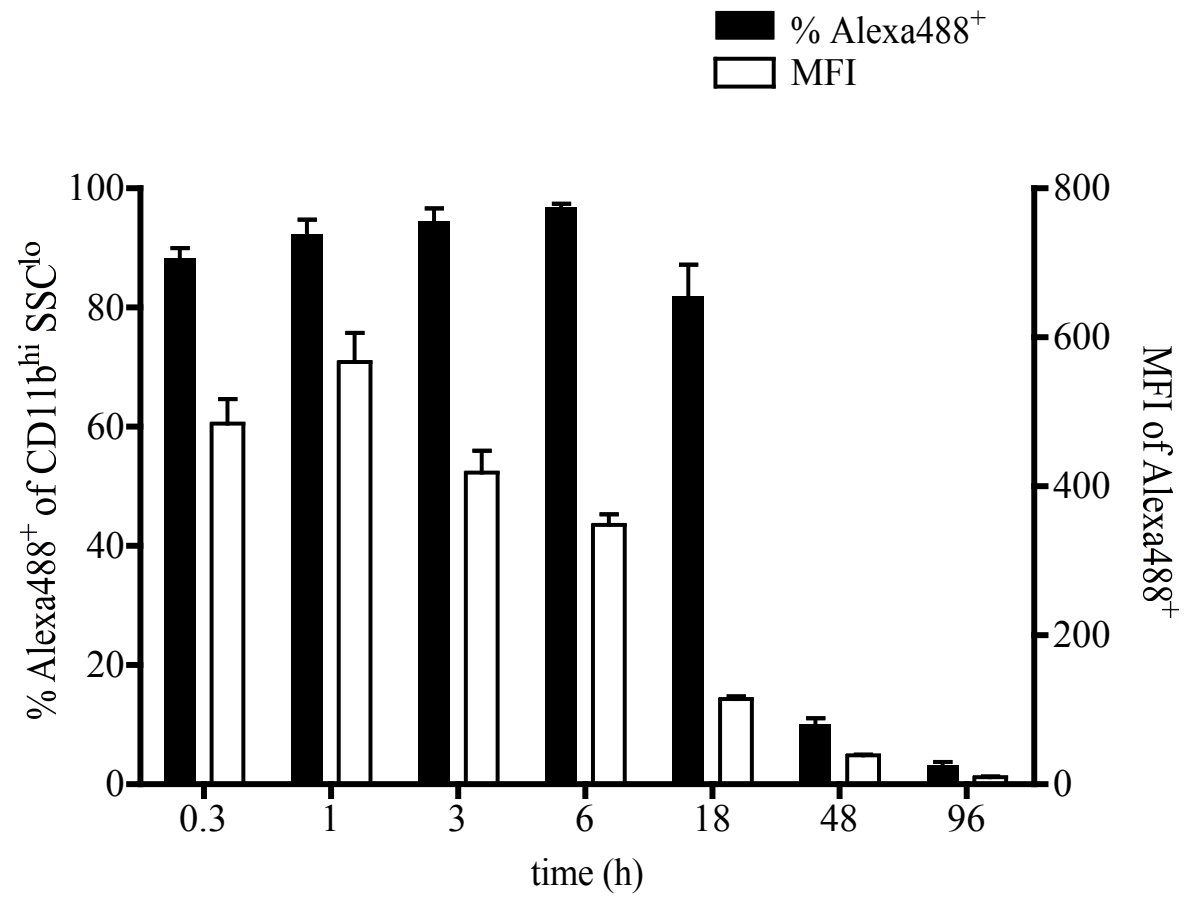

Figure 4.6: GA staining of blood monocytes is stable over 18 hours.

C57BL/6J mice were intravenously injected with $50 \mu \mathrm{g}$ of GA-Alexa488. Mice were tailbled at the indicated time points after the injections and blood cells were analysed by flow cytometry. Data originates from separate experiments for time ranges of 20 minutes to six hours and from six hours to 96 hours. Both experiments were performed twice with five mice each. 
Essentially the same percentage of monocytes was $\mathrm{GA}^{+} 20$ minutes after injection. The percentage of $\mathrm{GA}^{+}$monocytes remained stable for six hours, and decreased only slightly between six and 18 hours after injection, but the mean fluorescence index (MFI) of $\mathrm{GA}^{+}$cells declined gradually after one hour. After 48 hours, only a low percentage of monocytes could be identified as $\mathrm{GA}^{+}$, and the MFI had declined further. Two separate experiments were performed to cover the time periods from 20 minutes to six hours, and from six hours to 96 hours, respectively. Due to ethical regulations, tail bleeding was limited to four times for every individual mouse.

In summary, GA was found to rapidly bind to blood monocytes in an MHCII independent manner after intravenous injection. To determine the specificity of this binding, other proteins were tested for their ability to bind to monocytes Trypsinogen and DM SMEZ2, but not MOG or OVA bound to blood monocytes, indicating that GA binding to monocytes was not an artefact of non-specific binding. Although these results suggested that GA may specifically target monocytes whether GA binding alters monocyte function remained unclear.

\subsubsection{Intravenous GA Treatment Enhances the Suppressive Ability of Monocytes}

Immunosuppressive MDSCs with monocyte-like phenotype have been described in murine tumour models $(140,143)$ as well as in EAE (133). In addition, independent studies in our laboratory have shown that blood monocytes from naïve mice can suppress $\mathrm{T}$ cell proliferation in an antigen non-specific manner (C. Slaney, unpublished data). To determine if $\mathrm{T}$ cell suppression by monocytes could play a role in GA treatment, the effect of GA treatment on the suppressive ability of blood monocytes was investigated.

To test the suppressive ability of GA-treated blood monocytes, C57BL/6J mice were injected intravenously with GA, and PBMCs were recovered through density gradient separation of blood isolated three hours after GA treatment. Monocytes were enriched by magnetic sorting and co-cultured with C57BL/6J splenocytes in presence of beads coated with anti-CD3/anti-CD28 antibodies that promote polyclonal $\mathrm{T}$ cell expansion. Monocytes from naïve mice suppressed $\mathrm{T}$ cell proliferation in a dosedependent manner, and monocytes from GA treated mice were even more potent suppressors of $\mathrm{T}$ cell proliferation (figure 4.7). Suppression was not simply due to phagocytosis of the beads by monocytes, because the same effect was also observed in anti-CD3/anti-CD28 coated wells (C. Slaney, unpublished data). CFSE dilution assays also showed that proliferation of both CD4 and CD8 T cells in the mixed splenocyte 
culture was suppressed by monocytes (C. Slaney, unpublished data). These results suggested that the binding of GA to monocytes in blood could have functional relevance, by enhancing the potential of monocytes to suppress $\mathrm{T}$ cell dependent immune responses.

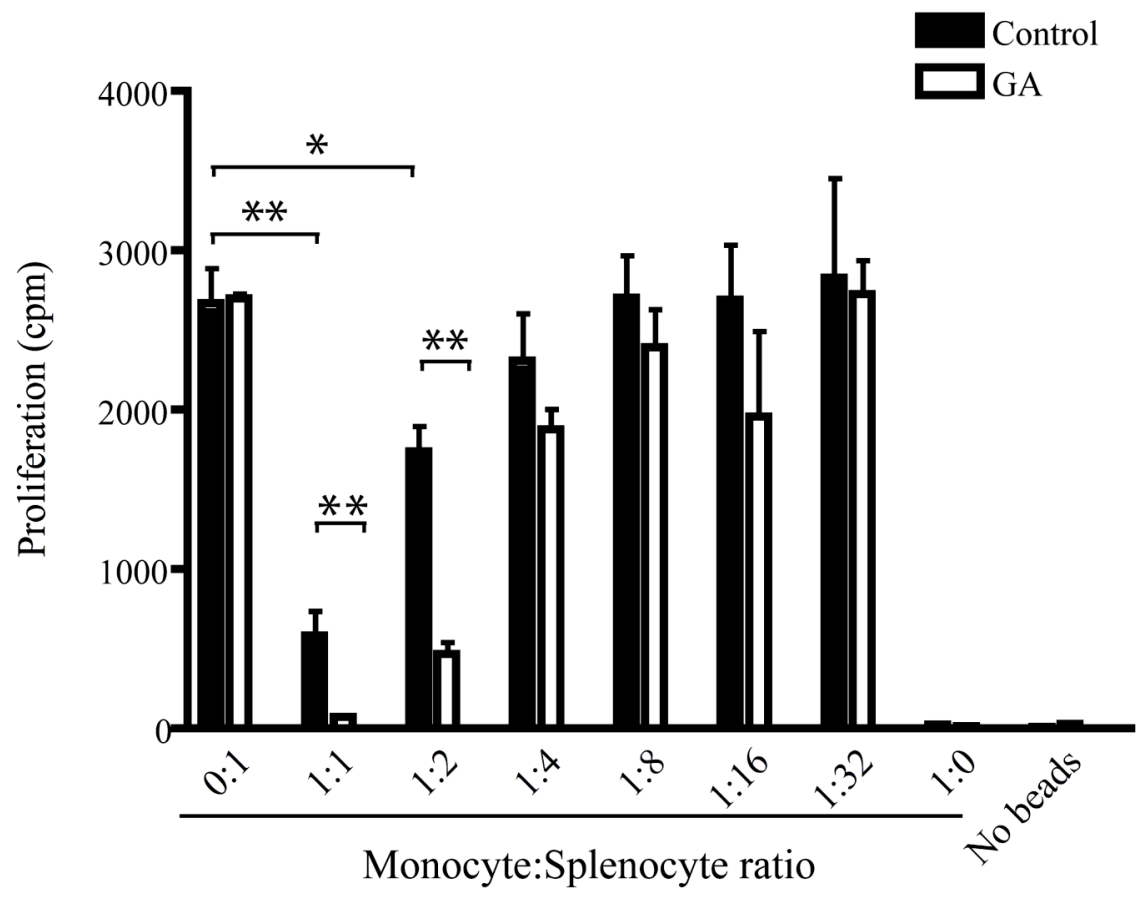

Figure 4.7: GA enhances the ability of monocytes to suppress polyclonal $\mathrm{T}$ cell proliferation.

C57BL/6J mice received intravenous injections of $50 \mu \mathrm{g}$ GA or were left untreated (control). PBMCs were recovered three hours after the injections, and CD11b $b^{\text {hi }}$ monocytes enriched by magnetic sorting $\left(>80 \% \mathrm{CD} 11 \mathrm{~b}^{\mathrm{hi}}\right)$. The monocytes were cocultured with $5 \times 10^{4} \mathrm{C} 57 \mathrm{BL} / 6 \mathrm{~J}$ splenocytes at the indicated ratios in presence of beads coated with anti-CD3/anti-CD28 in round-bottom wells. The cells were incubated for 24 hours, and additional eight hours in the presence of $\left[{ }^{3} \mathrm{H}\right]$-thymidine. Proliferation was measured by $\left[{ }^{3} \mathrm{H}\right]$-thymidine incorporation. Results represent one out of two independent experiments (Mean \pm SEM of triplicate wells; $* \mathrm{p}<0.01$, two-tailed t-test).

To test whether GA treatment could also enhance the suppression of antigen specific $\mathrm{T}$ cell proliferation, monocytes were enriched from PBMCs of GA treated mice or of naïve controls. The monocytes were co-cultured with CFSE labelled TCR transgenic 2D2 lymphocytes in the presence of $\mathrm{MOG}_{35-55}$ peptide. CD4 $\mathrm{T}$ cell proliferation in the co-culture was assessed by flow cytometric analysis of CFSE dilution. Monocytes of GA treated mice were equally potent suppressors of $\mathrm{MOG}_{35-55}$ specific CD4 $\mathrm{T}$ cell proliferation as naïve monocytes (figure 4.8). The failure to detect superior 
suppression by GA treated monocytes could have been due to limitations of the experimental setting, as discussed in section 4.3.3.

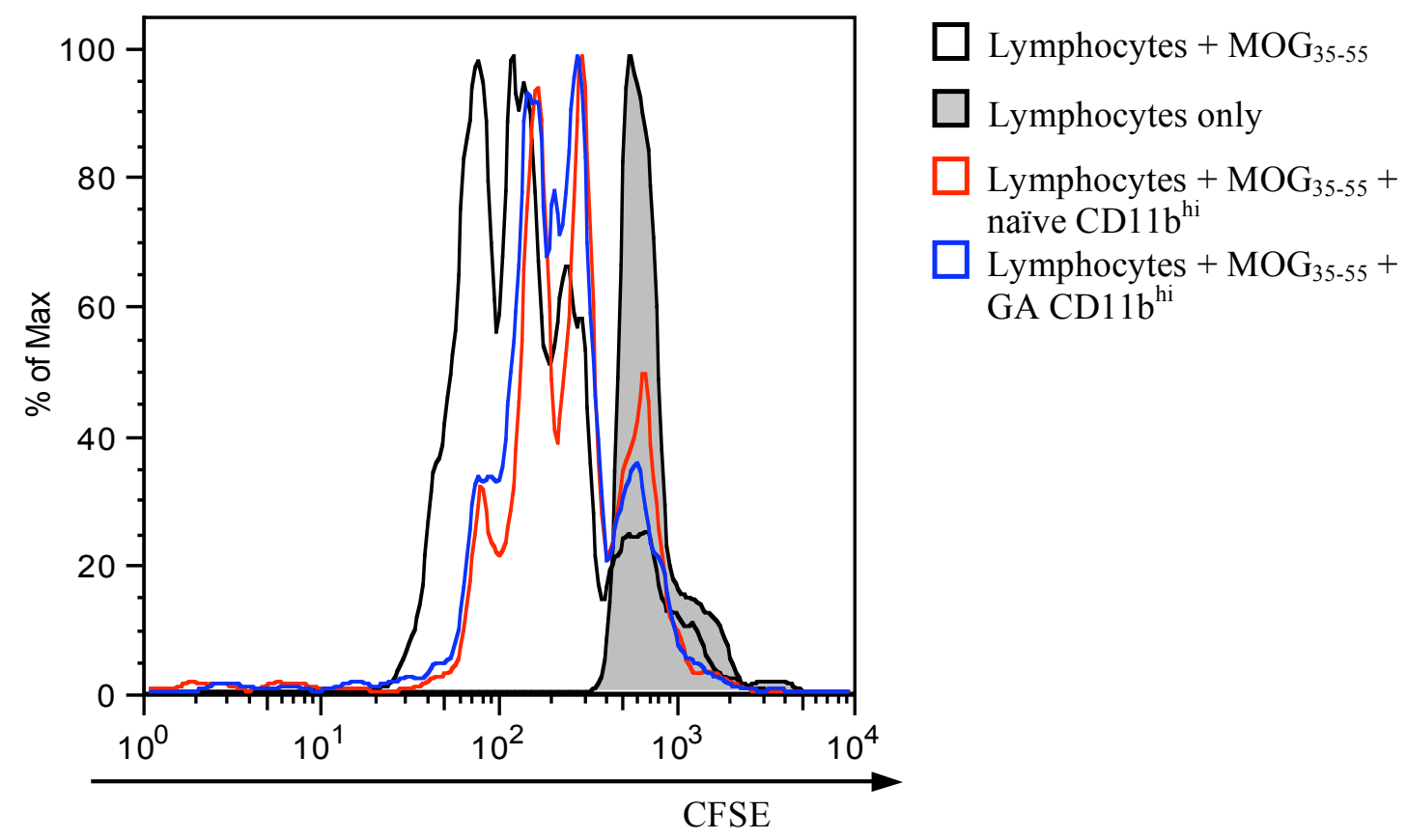

Figure 4.8: GA treated and naïve monocytes equally suppress $M_{0 G} O_{35-55}$ specific $T$ cell proliferation in vitro.

C57BL/6J mice received intravenous injections of $50 \mu \mathrm{g}$ GA or were left untreated. PBMCs were recovered three hours after the injections, and CD11 bi monocytes enriched by magnetic sorting $\left(>80 \% \mathrm{CD} 11 \mathrm{~b}^{\mathrm{hi}}\right)$. Monocytes from GA treated (blue histogram) or untreated (red histogram) mice were co-cultured with $5 \times 10^{4} \mathrm{C} 57 \mathrm{BL} / 6 \mathrm{~J}$ lymphocytes in presence of $10 \mu \mathrm{g} / \mathrm{ml} \mathrm{MOG}_{35-55}$ in round-bottom wells. The ratio of monocytes to lymphocytes was 1:2. 2D2 lymphocytes were cultured in presence (open black histogram) or absence (shaded histogram) of $\mathrm{MOG}_{35-55}$ as controls. The cells were cultured for 72 hours, and CFSE dilution was analysed by flow cytometry. Results represent one out of two independent experiments with duplicate wells.

To further investigate the possible role of $\mathrm{T}$ cell suppression in GA treatment, an proliferation assay was performed. $\mathrm{CD}^{+}$cells were purified from $2 \mathrm{D} 2$ mice, labelled with CFSE, and adoptively transferred to congenic mice, which express the CD45.1 marker on their leukocytes rather than CD45.2, which is expressed by C57BL/6J mice (and 2D2 mice, which are on C57BL/6J background). Together with the cell transfer, mice received intravenous injections of either $\mathrm{MOG}_{35-55}$ alone or $\mathrm{MOG}_{35-55}$ and GA. Analysis of CFSE dilution in spleens and blood of the recipients showed that 
$\mathrm{MOG}_{35-55}$ specific proliferation of TCR transgenic donor cells was reduced in GA treated mice, in comparison to mice injected with the MOG peptide only (figure 4.9).
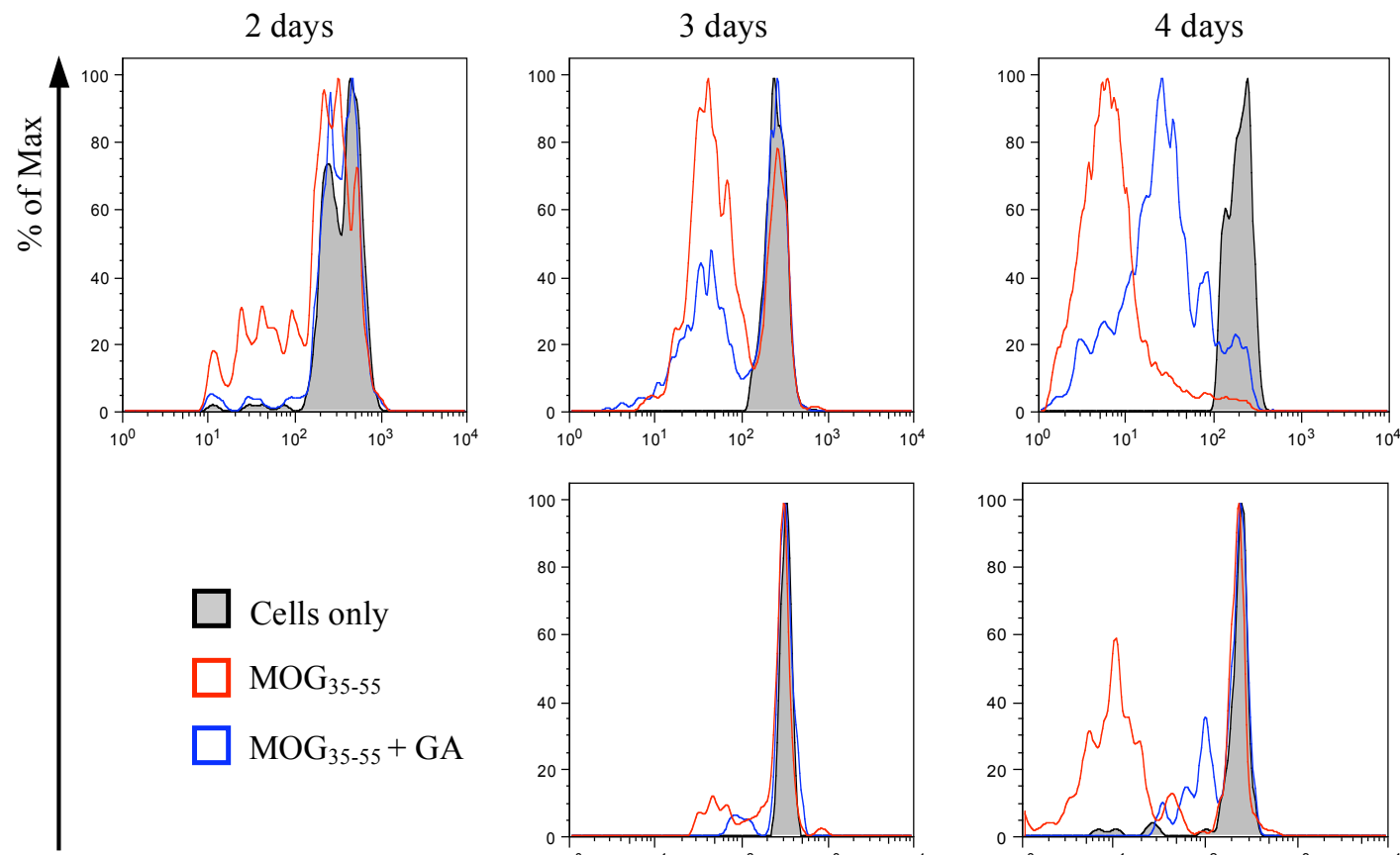

Spleen
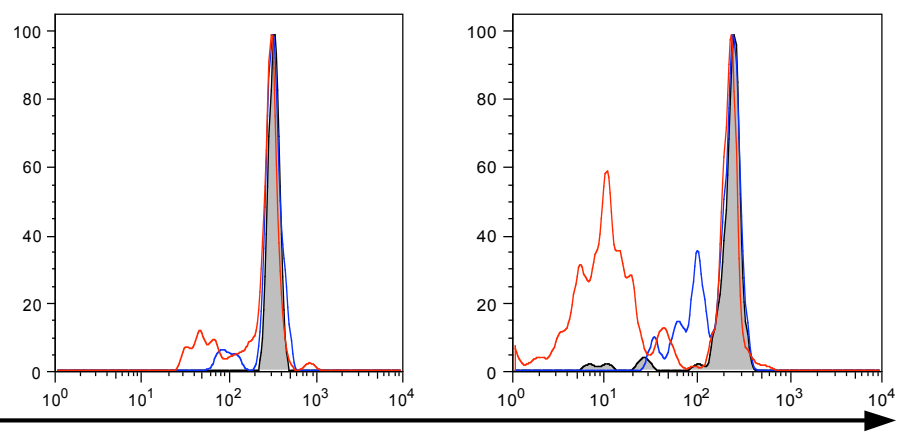

Blood

CFSE

Figure 4.9: Intravenous GA treatment suppresses CD4 T cell proliferation in vivo.

Splenocytes from 2D2 mice were labelled with CFSE and $\mathrm{CD}^{+}$cells were purified by magnetic sorting. Purified $\mathrm{CD}^{+}$cells were adoptively transferred to CD45-congenic mice $\left(4 \times 10^{6}\right.$ cells per mouse, $\left.>95 \% \mathrm{CD}^{+}\right)$. Mice were either injected with cells only (shaded histograms), with cells and $2 \mu \mathrm{g} \mathrm{MOG}_{35-55}$ (red histograms) or with cells and 2 $\mu \mathrm{g} \mathrm{MOG}_{35-55}$ and $50 \mu \mathrm{g}$ GA (blue histograms). Two to four days after cell transfer, CFSE dilution in the spleens and blood of the recipients was analysed by flow cytometry. Histograms are gated on CD $45.2^{+} \mathrm{CD} 4^{+}$cells. Data represents one out of two independent experiments ( $n=2$ mice per group).

These results showed that intravenous GA treatment could modulate monocytes to exhibit an increased capacity to suppress polyclonal, but not $\mathrm{MOG}_{35-55}$ specific, $\mathrm{T}$ cell proliferation . However, $\mathrm{MOG}_{35-55}$ specific $\mathrm{T}$ cell proliferation was impaired upon intravenous GA treatment. It is therefore possible that suppression of myelinspecific T cells by GA modulated monocytes contributes to GA therapy in EAE. 


\subsubsection{Effects of GA on Monocytes cannot be Detected in Draining Lymph Nodes}

Enhanced suppressive ability of GA treated monocytes was observed after intravenous injection of GA (section 4.2.2), but it remained unclear whether increased T cell suppression contributed to suppression of EAE in the subcutaneous co-immunisation GA treatment model.

The first question addressed was whether binding of GA to monocytes could be detected after subcutaneous injection. For this, C57BL/6J mice were subcutaneously immunised with $500 \mu \mathrm{g}$ FITC-labelled GA emulsified in CFA. Draining (inguinal) lymph node cells were analysed by flow cytometry two to four days after immunisation (figure 4.10). A small population of CD11b $b^{\text {hi }}$ cells (0.9-1.9\%) was present in the lymph nodes of immunised as well as naïve mice, but these cells did not show GA staining. GA staining could not be detected after subcutaneous injection in PBS either (data not shown).

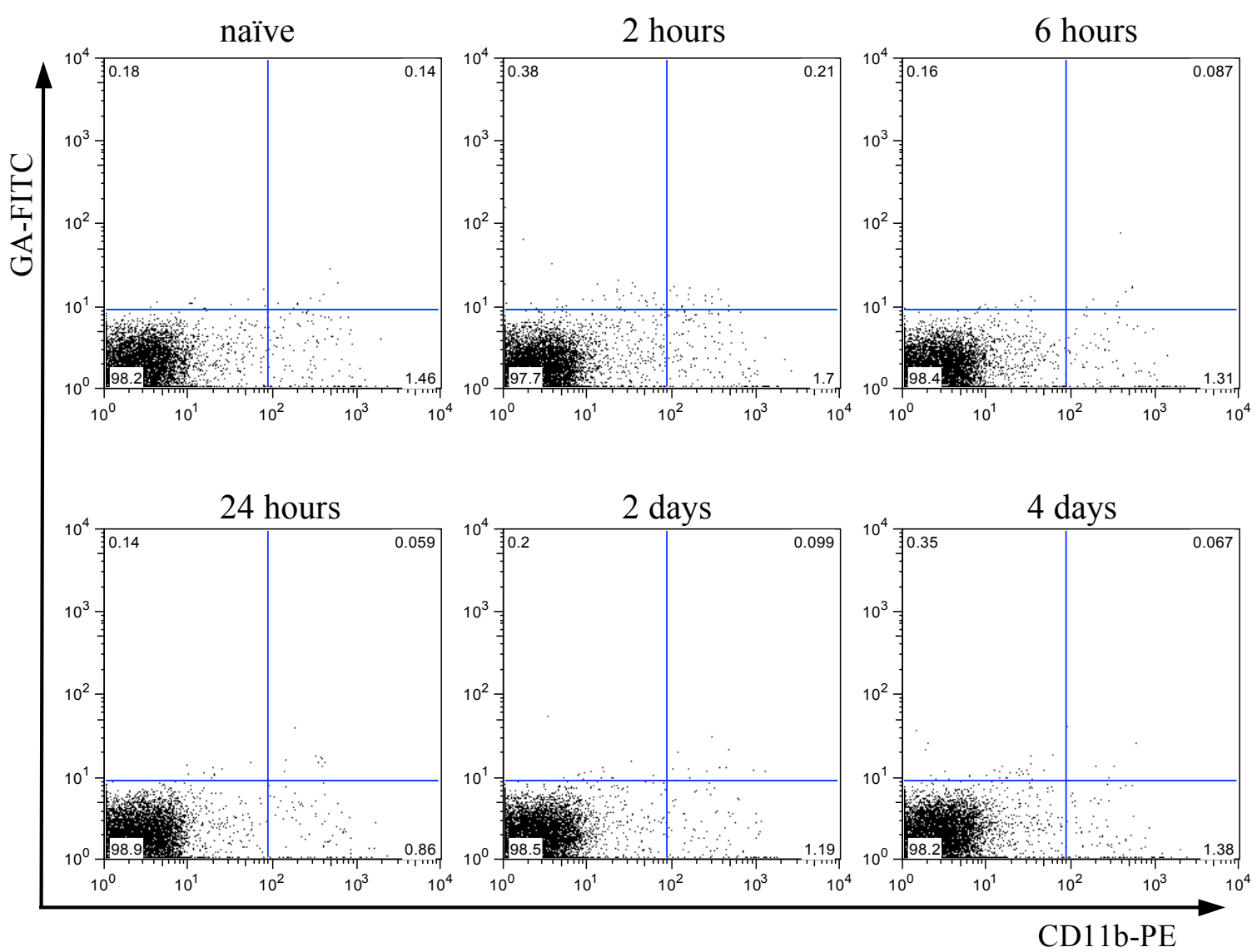

Figure 4.10: GA-FITC cannot be detected in lymph nodes after immunisation.

C57BL/6J mice were subcutaneously immunised with $500 \mu \mathrm{g}$ GA-FITC in CFA. Draining lymph nodes were digested with DNase/collagenase (see Materials \& Methods) at indicated time points after immunisation cells of individual mice were analysed by flow cytometry. Data represents one out of three experiments $(n=2$ mice per time point). 
The constant percentage of CD $11 b^{\text {hi }}$ cells in draining lymph nodes suggested that monocyte infiltration of draining lymph nodes did not occur within the first four days after immunisation. A more extensive time course experiment was performed to study the $\mathrm{CD} 11 \mathrm{~b}^{\text {hi }}$ population in the draining lymph nodes of immunised mice. The percentage of CD11b ${ }^{\text {hi }}$ cells did not change in draining lymph nodes during eight days following immunisation with CFA containing unlabelled GA (figure 4.11).
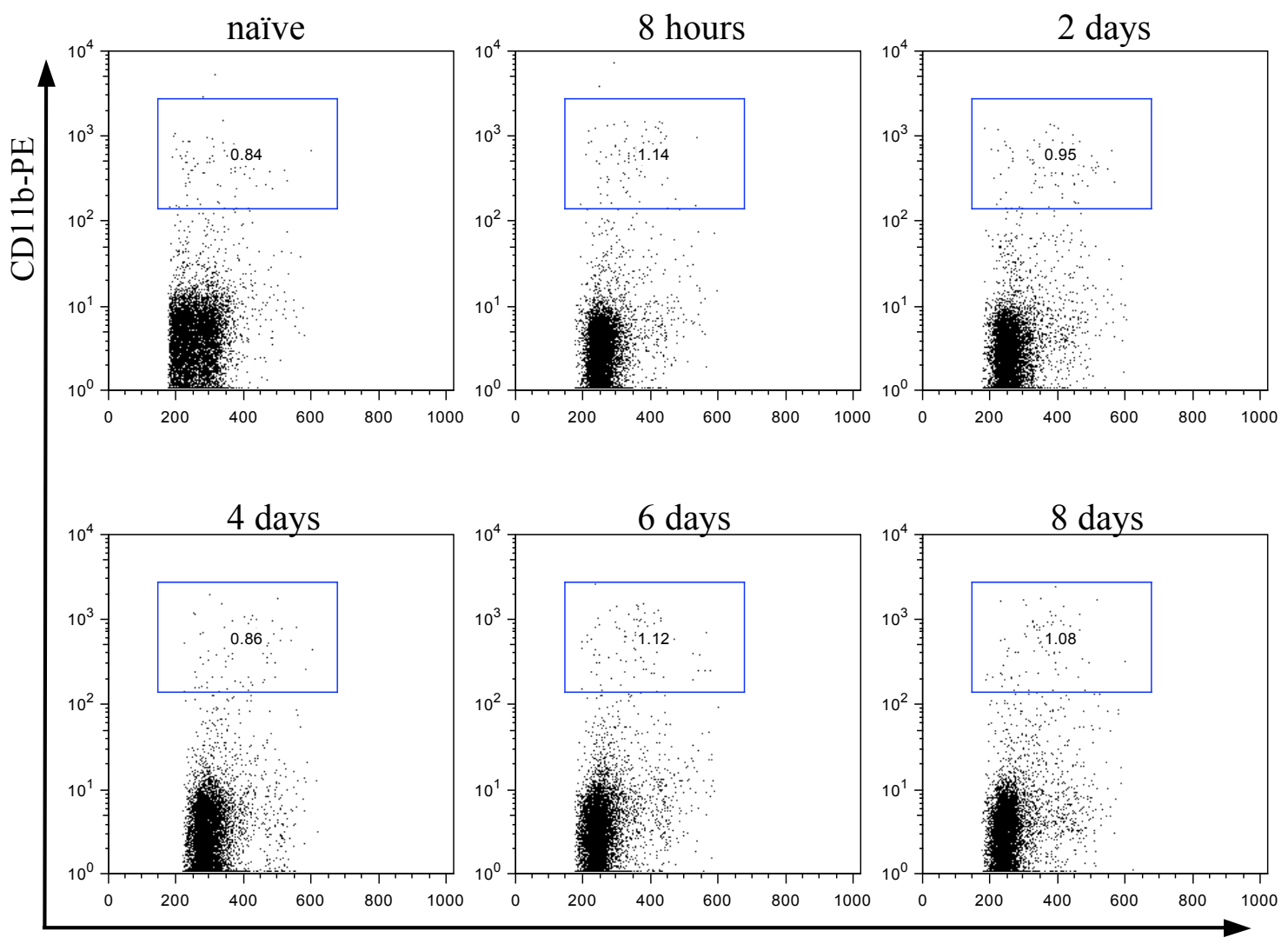

FSC

Figure 4.11: The percentage of $C D 11 b^{\text {hi }}$ cells in the draining lymph node does not change after immunisation.

C57BL6/J mice were immunised with $500 \mu \mathrm{g}$ GA emulsified in CFA Draining lymph nodes were digested with DNase/collagenase at indicated time points after immunisation and single cell suspensions of individual mice were analysed by flow cytometry. Data represents one out of four independent experiments $(n=2$ mice per time point $)$.

In CFA-immunised mice, accumulation of $\mathrm{CD}_{11} 1 \mathrm{~b}^{\text {hi }}$ cells was observed in the blood and spleen. The accumulating cells were CD11b ${ }^{\text {hi }}$ Ly6C ${ }^{\text {int }} \mathrm{Ly}_{6} \mathrm{G}^{+}$(figure 4.12), suggesting that they were neutrophils or other granulocytes (140). An increase in the 
$\mathrm{SSC}^{\text {lo }} \mathrm{CD} 11 \mathrm{~b}^{\text {hi }}$ monocyte population was not observed in spleens or blood of immunised mice.
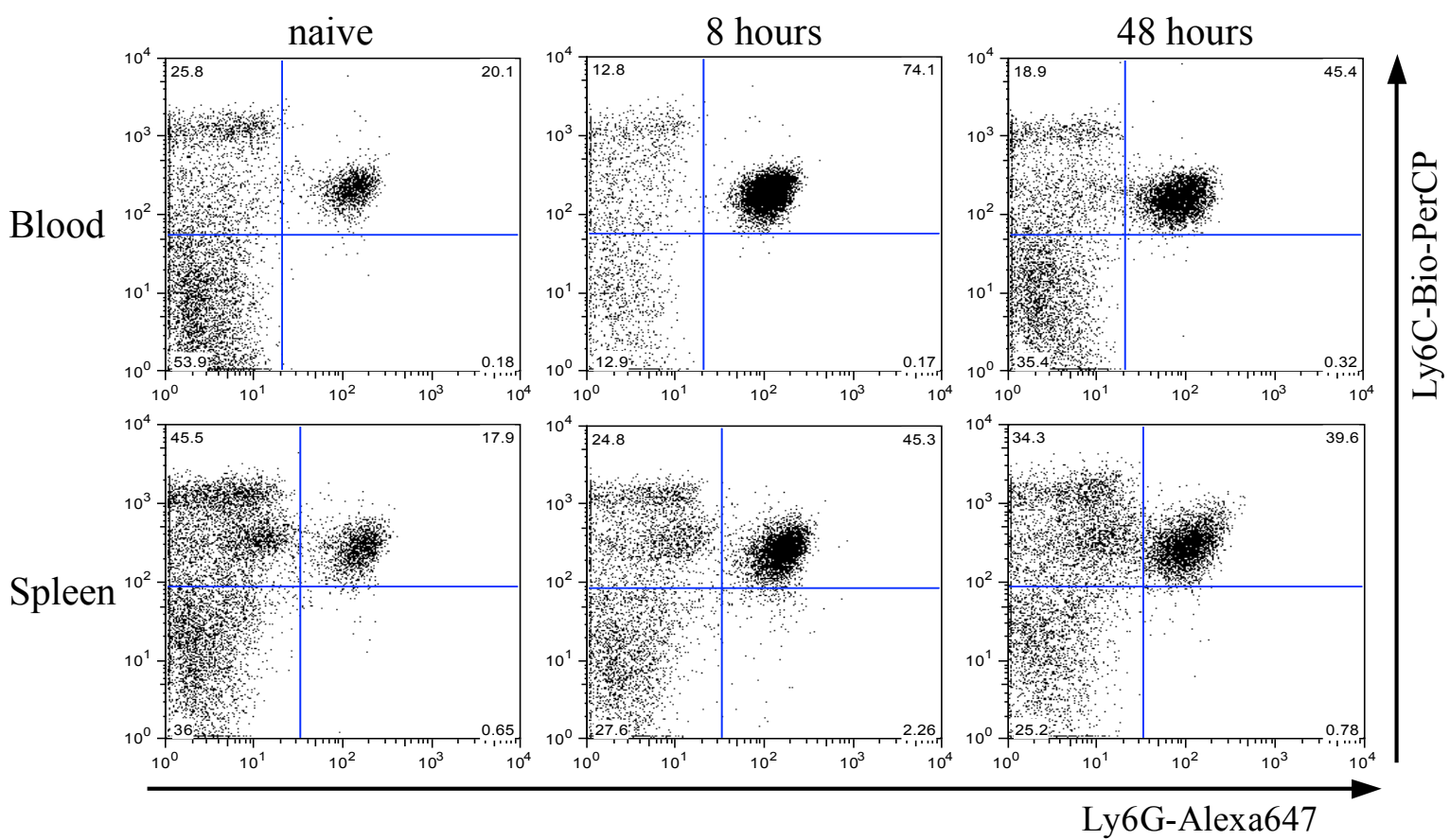

Figure 4.12: $\mathrm{CD}_{11 b^{\mathrm{hi}}} \mathrm{Ly6G}^{+}$granulocytes accumulate in spleen and blood after immunisation with CFA.

C57BL/6J mice were subcutaneously immunised with CFA. Single cell suspensions from spleens and blood were prepared eight or 48 hours after immunisation and analysed by flow cytometry. Plots are gated on $\mathrm{CD} 11 \mathrm{~b}{ }^{\text {hi }}$ cells. Data represents one out of four independent experiments ( $\mathrm{n}=2$ mice per time point).

The lack of monocyte accumulation in draining lymph nodes after immunisation raised the question whether blood monocytes escaped detection because they had undergone phenotypic changes following immunisation, for instance matured into DCs. To address this question, enriched CD11 bi monocytes from naïve C57BL/6J mice were adoptively transferred to naïve CD45-congenic mice. One day after cell transfer, recipients were immunised with CFA. Cells expressing CD45.2 were not detected in draining lymph nodes of recipients five days after cell transfer (figure 4.13). However, CD $45.2^{+}$cells could not be detected in the blood of the recipients, either. The small percentage of seemingly CD $45.2^{+} \mathrm{CD} 11 \mathrm{~b}^{+}$cells observed in both tissues were likely to be auto-fluorescent cells, because they were also present in tissues from mice that had not received cell transfer (figure 4.13). 


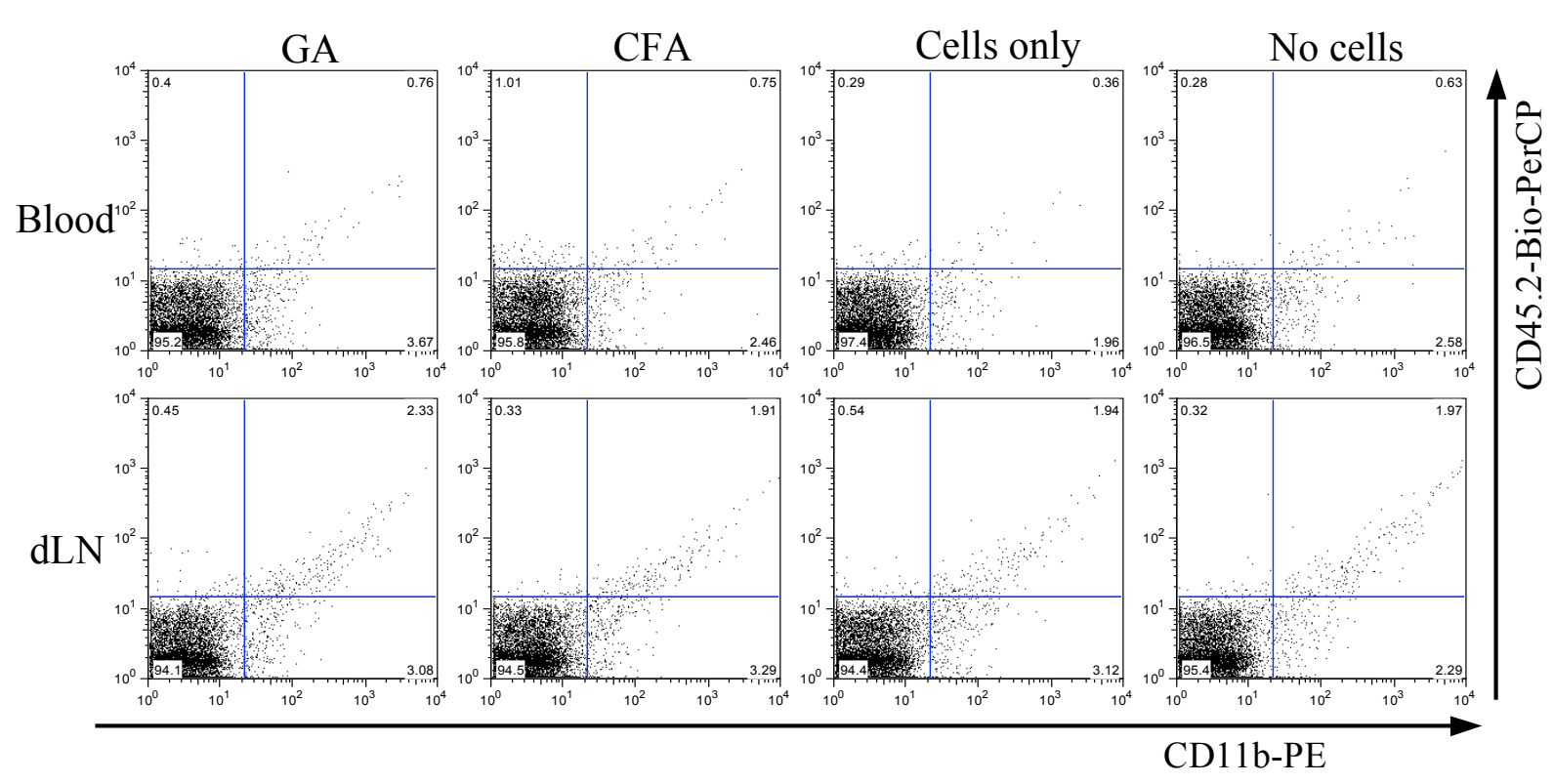

Figure 4.13: Adoptively transferred monocytes cannot be detected in draining lymph nodes of immunised recipients.

PBMCs from C57BL/6J mice were enriched for $\mathrm{CD} 11 \mathrm{~b}^{\text {hi }}$ monocytes by magnetic sorting $\left(>80 \% \mathrm{CD} 11 \mathrm{~b}^{\mathrm{hi}}\right)$. The monocytes were adoptively transferred to CD45-congenic mice ( $2 \times 10^{5}$ cells per mouse). One day after cell transfer, recipients were immunised with $500 \mu \mathrm{g}$ GA emulsified in CFA, or CFA only, or were not immunised (cells only). Four days after immunisations, cells were isolated from draining lymph nodes (dLN) and the blood and analysed by flow cytometry. Data represents one out of two independent experiments ( $\mathrm{n}=1$ mouse per group).

In summary, monocyte infiltration could not be detected in draining lymph nodes of immunised mice. The lack of monocyte infiltration was evident over eight days following immunisation, and adoptively transferred $\mathrm{CD} 11 \mathrm{~b}^{\text {hi }}$ cells could not be recovered from draining lymph nodes or blood of immunised congenic recipients.

\subsubsection{GA Treatment does not Impair T Cell Proliferation in EAE}

The lack of monocyte infiltration of the draining lymph nodes after immunisation raised the question whether increased $\mathrm{T}$ cell suppression by monocytes after intravenous GA treatment would translate to the subcutaneous treatment model. To clarify whether suppression of myelin specific T cells contributes to GA therapy in EAE, an and an approach was undertaken.

First, the proliferative capability of lymphocytes from GA treated mice was compared to untreated mice. For this, EAE was induced in C57BL/6J mice, and the 
mice were either left untreated or co-immunised with GA. Seven days later, lymphocytes were isolated from draining lymph nodes of immunised mice and cultured in presence of $\mathrm{MOG}_{35-55}$. In this setting, lymphocytes from GA treated mice did not show reduced proliferation in comparison to untreated mice (figure 4.14).

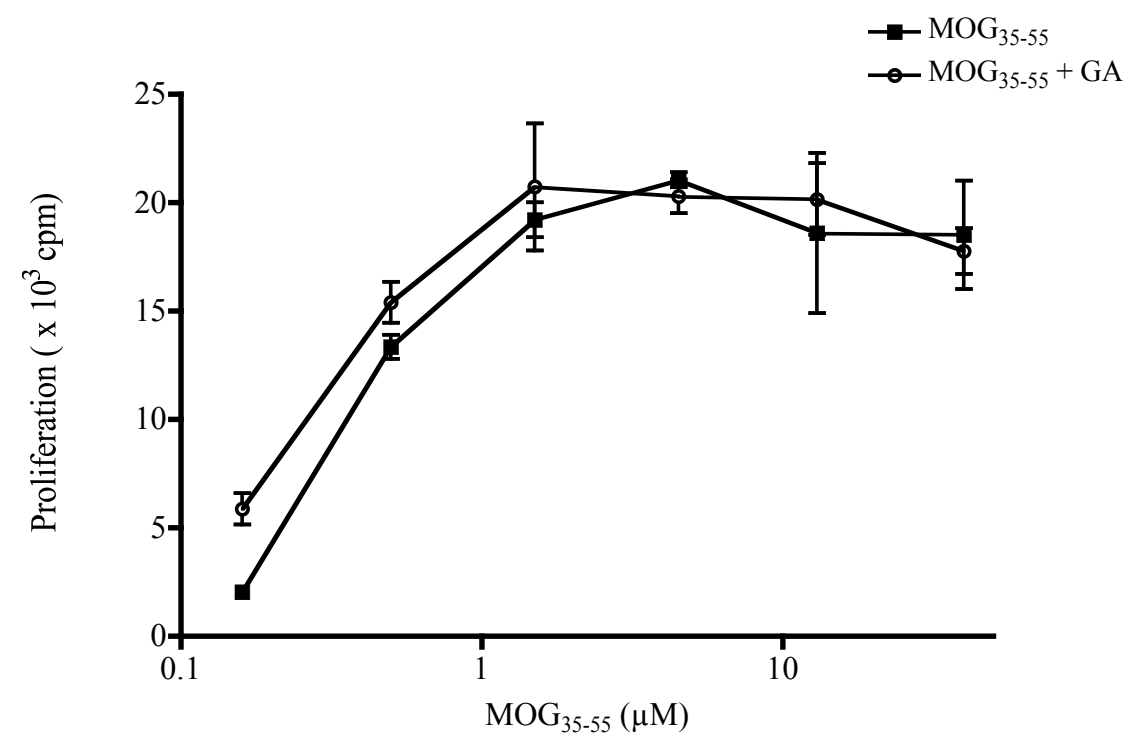

Figure 4.14: Co-immunisation with GA does not impair ex vivo MOG $_{35-55}$ specific $T$ cell proliferation.

C57BL/6J mice were immunised with $\mathrm{MOG}_{35-55}$ or GA treated by co-immunisation $\left(\mathrm{MOG}_{35-55}+\mathrm{GA}\right)$. Cells were isolated from draining lymph nodes seven days after immunisations and incubated in presence of $\mathrm{MOG}_{35-55}\left(5 \times 10^{5}\right.$ cells per well). The cells were incubated for 80 hours, and additional 16 hours in presence of $\left[{ }^{3} \mathrm{H}\right]$-thymidine. Proliferation was measured by $\left[{ }^{3} \mathrm{H}\right]$-thymidine incorporation $\left(\mathrm{p}>0.1\right.$ for $\mathrm{MOG}_{35-55}$ vs. $\mathrm{MOG}_{35-55}+\mathrm{GA}$ ). Data represents one out of two independent experiments (Mean $\pm \mathrm{SEM}$ of triplicate wells).

Secondly, $\mathrm{MOG}_{35-55}$ specific $\mathrm{T}$ cell proliferation was investigated in GAtreated mice. CFSE labelled $\mathrm{CD}^{+}$cells from 2D2 mice were adoptively transferred to CD45-congenic mice. One day after cell transfer, recipients were immunised with CFA only, with $\mathrm{MOG}_{35-55}$, or GA treated by co-immunisation. Two to four days after immunisation, CFSE dilution in donor cells was assayed in spleens, draining lymph nodes and blood of the recipients (figure 4.15). No significant differences in proliferation were detected in any of the analysed tissues between GA treated and untreated mice. 

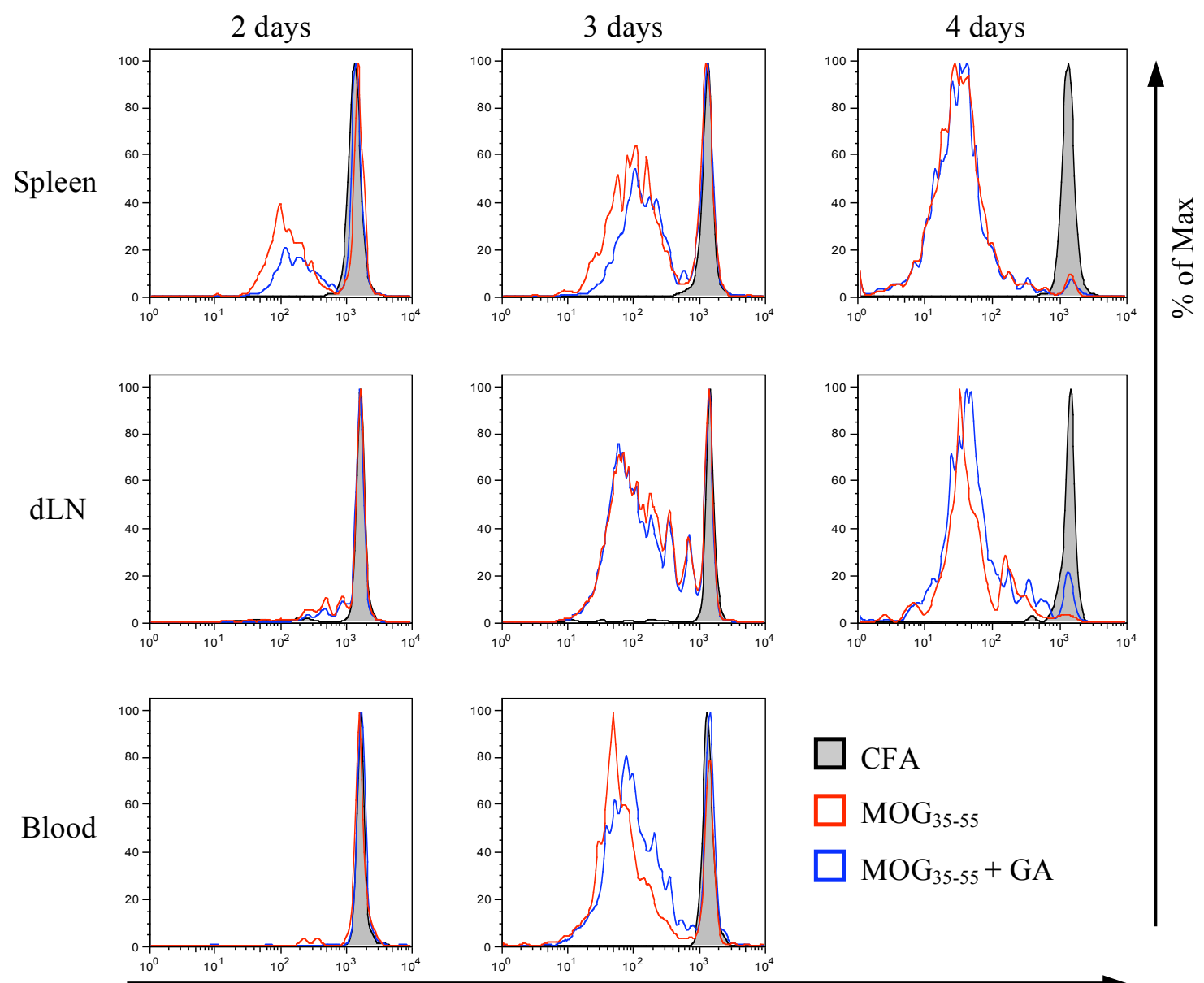

CFSE

Figure 4.15: Co-immunisation treatment with GA does not suppress $\mathbf{M O G}_{35-55}$ specific $\mathbf{T}$ cell proliferation in EAE.

Splenocytes from 2D2 mice were labelled with CFSE and $\mathrm{CD}^{+}$cells were purified by magnetic sorting. Purified $\mathrm{CD}^{+}$cells were adoptively transferred to CD45-congenic mice $\left(4 \times 10^{6}\right.$ cells per mouse, $\left.>96 \% \mathrm{CD}^{+}\right)$. One day after cell transfer, recipients were immunised with CFA only (shaded histograms), with $\mathrm{MOG}_{35-55}$ (red histograms) or GAtreated by co-immunisation (blue histograms). Two to four days after the immunisations, CFSE dilution in the spleens, draining lymph nodes (dLN) and blood of the recipients was analysed by flow cytometry. Histograms are gated on $\mathrm{CD} 45.2^{+} \mathrm{CD} 4^{+}$cells. Data represents one out of at least two independent experiments ( $n=2$ mice per group).

In summary, suppression of $\mathrm{MOG}_{35-55}$ specific $\mathrm{T}$ cell proliferation was not observed in mice co-immunised with GA. These results are in contrast to the enhancement of suppressive ability of monocytes and reduced

$\mathrm{MOG}_{35-55}$ specific CD4 $\mathrm{T}$ cell proliferation following intravenous GA treatment (see figures 4.7 and 4.9), and suggest that suppression of myelin specific $\mathrm{T}$ cells does not occur after subcutaneous co-immunisation with GA. 


\subsubsection{Temporary Monocyte Depletion does not Impair GA Treatment of EAE}

Enhanced T cell suppressive ability of monocytes after intravenous GA treatment did not translate into increased suppression of myelin reactive $\mathrm{T}$ cells in the subcutaneous treatment model. However, monocytes have been shown to contribute to GA treatment in EAE by inducing regulatory and anti-inflammatory $\mathrm{T}$ cell responses (80). Furthermore, the binding assays presented in section 4.2.1 suggested that GA may preferentially target monocytes in EAE treatment Therefore the next question to be addressed was whether therapeutic efficacy of GA in EAE is dependent on monocytes.

Monocytes can be depleted and with dichloromethylene diphosphonate $\left(\mathrm{Cl}_{2} \mathrm{MDP}\right)$ loaded liposomes, which are taken up by phagocytic cells and induce their apoptosis (144). To investigate whether the presence of monocytes is essential for efficacy of GA treatment, monocytes of C57BL/6J mice were depleted by intravenous injection of $\mathrm{Cl}_{2} \mathrm{MDP}$-liposomes prior to EAE induction and GA treatment. Monocytes comprised less than 1\% of PBMCs 24 hours after liposome injection, as opposed to $5-10 \%$ in mice that had not received liposomes (data not shown). GA treated mice were protected from EAE to the same extent, regardless of whether they had received monocyte depleting treatment or not (figure 4.16).

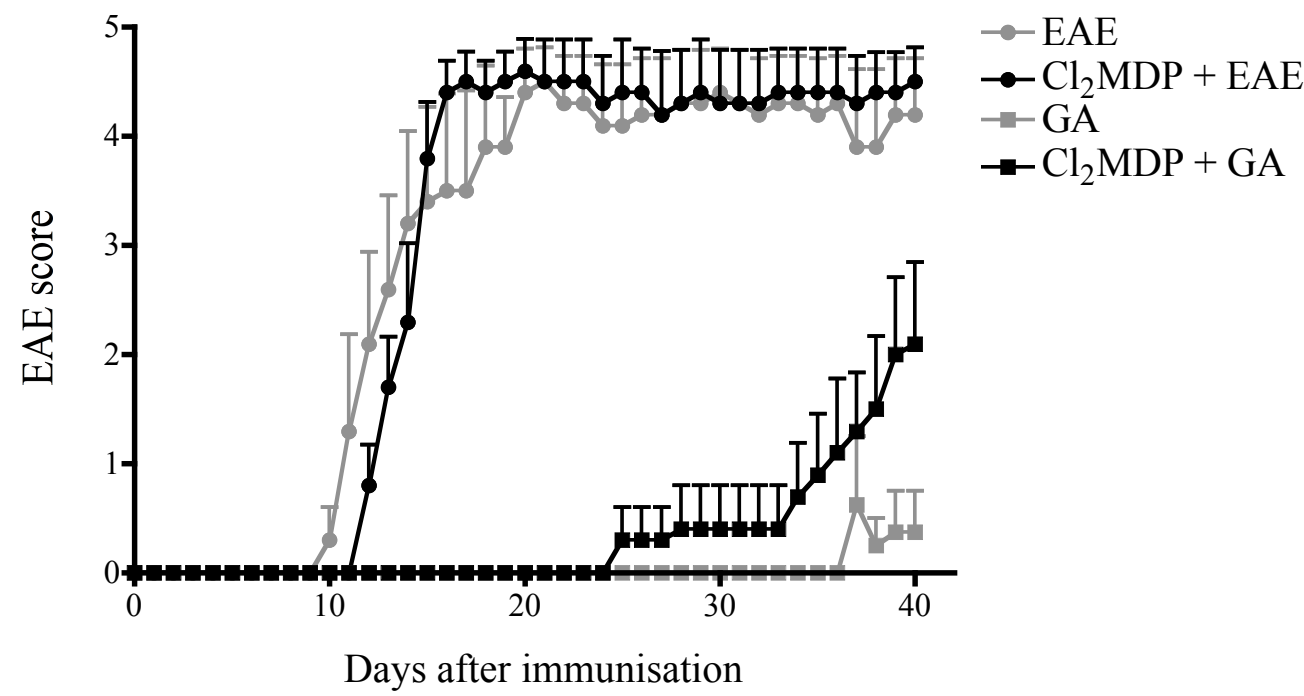

Figure 4.16: Temporary monocyte depletion does not impair GA treatment of EAE. $\mathrm{C} 57 \mathrm{BL} / 6 \mathrm{~J}$ mice received intravenous injections of $\mathrm{Cl}_{2} \mathrm{MDP}$-liposomes 16 hours before EAE induction and GA treatment by co-immunisation. $\left(\mathrm{p}<0.001\right.$ for $\mathrm{Cl}_{2} \mathrm{MDP}+\mathrm{EAE}$ vs. $\mathrm{Cl}_{2} \mathrm{MDP}+\mathrm{GA} ; \mathrm{p}>0.05$ for GA vs. $\mathrm{Cl}_{2} \mathrm{MDP}+\mathrm{GA}$; mean $\pm \mathrm{SEM}$ of $\mathrm{n}=5$ mice per group). Data represents one out of two independent experiments. 
GA-treated mice that received $\mathrm{Cl}_{2} \mathrm{MDP}$ developed slightly more severe EAE than GA-treated mice without monocyte depletion. This difference occurred 25-30 days after monocyte depletion and was not statistically significant. This slight difference is therefore unlikely to be attributable to the temporary depletion of monocytes.

Monocyte depletion did not interfere with the onset or the progression of EAE in untreated control mice. Depletion of monocytes by $\mathrm{Cl}_{2} \mathrm{MDP}$ is only transient, and monocyte numbers recover three to four days after their depletion (131). Hence, this finding showed that GA therapy did not depend on the presence of monocytes in the early phase of treatment.

\subsubsection{Targeting GA to CD11b does not Suppress EAE at Low Dose}

The association of GA with monocytes as well as its effects on suppressive function of monocytes after intravenous treatment did not translate to the subcutaneous co-immunisation model. In a reciprocal approach, intravenous injection of $\mathrm{C} 57 \mathrm{BL} / 6 \mathrm{~J}$ mice with a single dose of $500 \mu \mathrm{g}$ of GA immediately after EAE induction failed to suppress disease (figure 4.17).

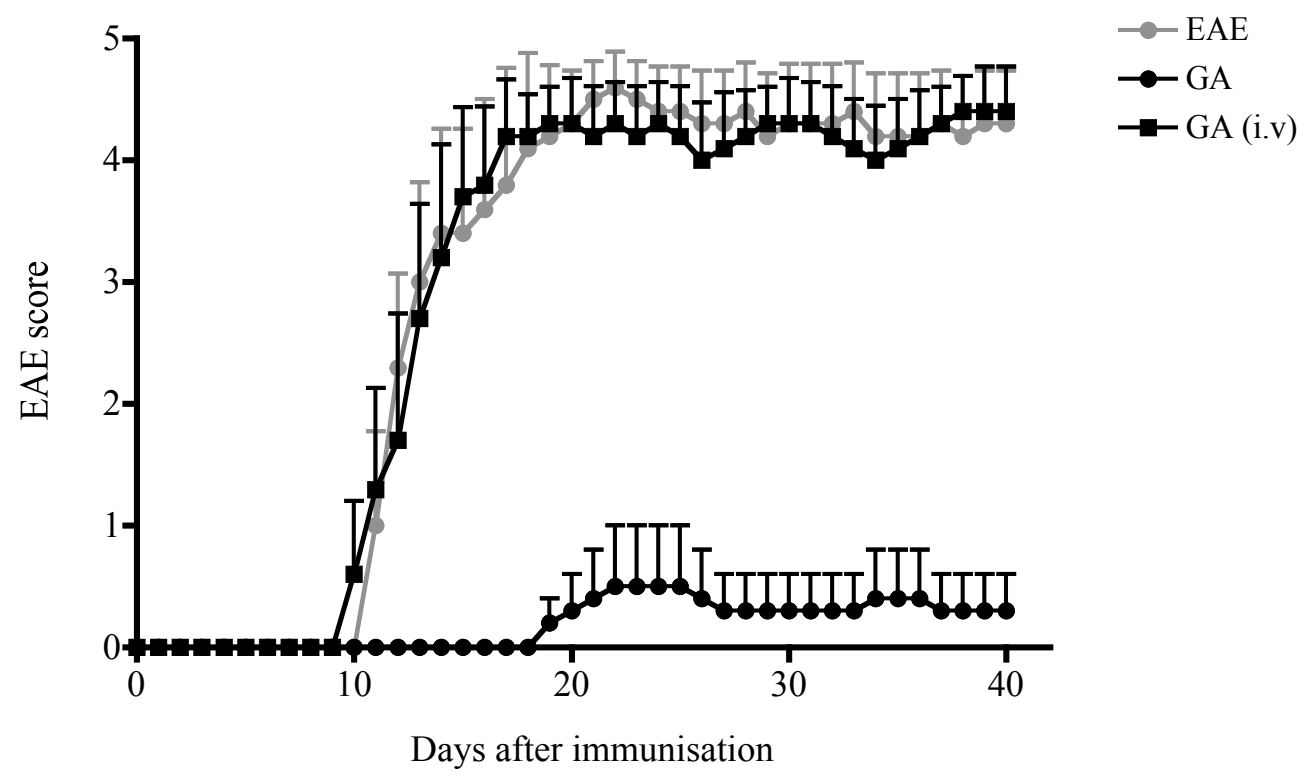

Figure 4.17: Intravenous GA treatment does not suppress EAE.

C57BL/6J mice received intravenous injections of $500 \mu \mathrm{g}$ GA in PBS immediately before EAE induction (GA (i.v)). Mice were left untreated (EAE) or co-immunised with GA as controls. ( $p>0.05$ for EAE vs. GA (i.v); mean \pm SEM of $n=5$ mice per group). Data represents one out of two independent experiments. 
The failure of intravenous GA treatment to suppress EAE may have been due to a lack of a sustained effect on monocytes after a single injection. Persistent effects of GA on the immune system is ensured by CFA in the subcutaneous treatment model, which gradually releases the drug into the system over time (37). Hence, a subcutaneous treatment strategy of targeting GA to monocytes was tested to assess whether sustained release of monocyte-targeted GA could suppress EAE.

For monocyte targeting, GA was conjugated to an antibody specific for CD11b (clone M1/70), a cell surface molecule that all GA-binding monocytes invariably expressed at high levels (see figure 4.4). The concentration of the conjugate was determined by $\mathrm{OD}_{280}$ measurement. For treatment of EAE, mice were co-immunised with $20 \mu \mathrm{g}$ of the conjugate (M1/70-GA). As shown in figure 4.18, mice that had been treated with M1/70-GA were not protected from EAE. Consistent with earlier findings (see figure 3.16), an equal molar amount of GA that corresponded to the molarity of the conjugate $(1.2 \mu \mathrm{g})$ also failed to ameliorate EAE.

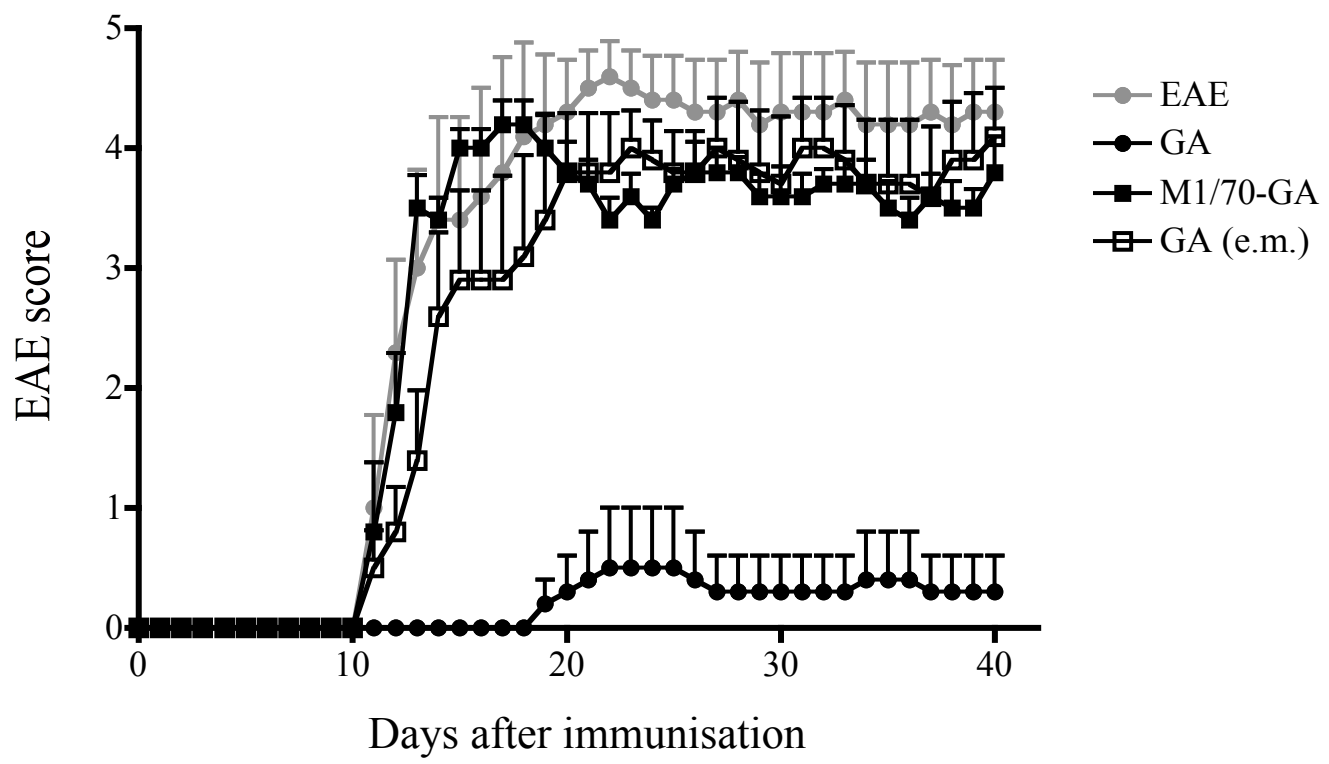

Figure 4.18: Targeting GA to CD11b does not suppress EAE.

C57BL/6J mice were left untreated (EAE), or treated with $20 \mu \mathrm{g} \mathrm{M1/70-GA,} \mathrm{or} \mathrm{with}$ $1.2 \mu \mathrm{g}$ GA (GA(e.m)) following the co-immunisation protocol. Mice were co-immunised with $500 \mu \mathrm{g}$ GA as a positive control. ( $\mathrm{p}>0.05$ for EAE vs. M1/70-GA, $\mathrm{p}>0.05$ for EAE vs. GA (e.m), p < 0.001 for GA vs. GA (e.m) and for GA vs. M1/70-GA; mean \pm SEM of $n=5$ mice per group). Data represents one out of two independent experiments. 


\subsubsection{Summary}

Fluorophor-labelled GA bound to a heterogeneous population of monocytes in the blood of mice after intravenous injection. The binding was independent of MHCII expression by the monocytes, and suggested that GA treatment may primarily target monocytes . GA binding to monocytes also altered monocyte function, since intravenous GA treatment enhanced the intrinsic ability of the monocytes to suppress polyclonal $\mathrm{T}$ cell proliferation in an antigen non-specific manner.

$\mathrm{MOG}_{35-55}$ specific $\mathrm{T}$ cell proliferation was also reduced after intravenous GA treatment. However, $\mathrm{MOG}_{35-55}$ specific CD4 $\mathrm{T}$ cell proliferation was not impaired in subcutaneous GA treatment of EAE, suggesting that direct suppression of $\mathrm{MOG}_{35-55}$ specific $\mathrm{T}$ cells by monocytes did not contribute to GA therapy in the co-immunisation treatment model.

Monocytes are known to play an important protective role in GA therapy of EAE in mice (80). It was shown in this chapter that temporary depletion of monocytes did not impair efficiency of GA treatment, which may have been due to the temporary nature of depletion. Alternatively, other cell types such as DCs may be able to initiate protective responses in a similar way to monocytes and to compensate for the loss of monocytes. Targeting GA to monocytes using a monoclonal antibody specific for CD11b was ineffective in suppressing EAE. This may have been due to the use of an inadequate dose of the conjugate or its sequestration by other CD11b ${ }^{\text {hi }}$ cells such as neutrophils. 


\subsection{Discussion}

Modulation of innate immune cell function is considered to be an important feature of GA treatment in EAE and MS (86). GA-modulated innate cells can act as APCs and induce anti-inflammatory or regulatory $\mathrm{T}$ cell responses (80). However, the mechanism by which GA alters APCs phenotype remains unknown. GA has been shown to directly bind to MHCII

(87), but there is currently no experimental evidence supporting that this binding to MHCII is responsible for changes in APC function. In addition, it is unclear whether GA preferentially acts on a particular subset of APCs or can modulate multiple APC subsets in a redundant manner. Finally, T cell independent mechanisms of immunosuppression by GA-modulated innate cells may also contribute to the inhibition of autoimmunity.

\subsubsection{GA Binding to Blood Monocytes in vivo}

Fluorophor labelled GA bound to a CD11 bi ${ }^{\text {hi }}$ cell population in blood and spleen after intravenous injection. The cell surface phenotype of these cells suggests that they are a heterogeneous population of monocytes and monocyte-derived cells (126). Some of the GA binding cells expressed CD11c or MHCII, suggesting that they may be monocytes in an intermediate state of differentiation into DCs (126). Alternatively, the $\mathrm{CD}_{11 \mathrm{c}^{+}}$cells may be resident monocytes, which have also been shown to express CD11c (131). In support of a resident monocyte phenotype, CD11c and MHCII mostly did not co-localise on the same $\mathrm{GA}^{+}$cells, and $\mathrm{CD} 11 \mathrm{c}^{+}$resident monocytes have been shown to lack MHCII expression (131).

Binding of fluorophor-labelled GA to CD11b expressing cells could not be detected (see figure 4.3). GA-Alexa488 was used at a concentration of $10 \mu \mathrm{M}$ $(100 \mu \mathrm{g} / \mathrm{ml})$ in the binding assays. With the assumption that mice have a total of at least one millilitre blood, the concentration of GA-Alexa488 in the bloodstream was $50 \mu \mathrm{g} / \mathrm{ml}$ after intravenous injection of $50 \mu \mathrm{g}$ GA-Alexa488. Hence, the lack of binding to monocytes was not due to a lower concentration of GA compared to the binding assays.

GA did not bind to MHCII expressing cells , either (see A1.4), in contrast to earlier findings $(87,88)$. The conflicting results may be attributable to the difference in antigen concentrations used in the two studies. Fridkis-Hareli used GA at a concentration of $750 \mu \mathrm{M}(87)$, which is much higher than the $10 \mu \mathrm{M}$ that was used in this 
study. Sequestration of labelled GA by other cells was not responsible for the failure to detect GA staining of $\mathrm{MHCII}^{+}$cells in this study, because the percentage of total cells that showed GA staining was very low (see figure 4.3 and A1.4). However, binding to MHCII itself may require high abundance of GA.

Incubation of cells with low dose GA better represents exposure of immune cells to the drug, because GA is quickly degraded by extracellular proteases after injection (62). Moreover, GA is administered in an adjuvant-containing emulsion in most murine models (107), so that it is slowly released in small amounts at a time. Hence it is not clear whether the binding to MHCII observed by Fridkis-Hareli at high abundance (87) has functional relevance towards GA treatment

The majority of $\mathrm{GA}^{+}$monocytes in the blood did not express MHCII, and GA also bound to monocytes in MHCII deficient mice (see figure 4.5), thus formally excluding participation of MHCII molecules in the binding of GA to monocytes. This finding is in further support of a recent study that reported an MHCII independent modification of monocyte function during GA treatment (80).

The lack of binding of GA to monocytes indicates that staining of blood monocytes may be dependent on the presence of an as yet unidentified soluble factor in the blood. This soluble factor may be a component of the complement system. For example, GA can bind to mononuclear cells via $\alpha_{M} \beta_{2}$ integrin (Mac-1, CD11b/CD18), and $\alpha_{M} \beta_{2}$ integrin can act as a complement receptor (145). $\alpha_{M} \beta_{2}$ integrin is expressed by blood monocytes and may be the target of GA on the surface of the monocytes. However, other soluble factors may also be responsible for GA binding to monocytes.

In addition to GA, DM SMEZ2 and trypsinogen also bound to blood monocytes after intravenous injection. DM SMEZ2 is taken up into intracellular compartments by blood monocytes (C. Slaney, unpublished data). Biotinylated GA could not be detected on the surface of the monocytes (see figure 4.2), suggesting that GA, too, may be internalised after binding to monocytes.

The mechanism by which GA associates with blood monocytes requires further study. Investigation of the precise mechanism was beyond the scope of this study and was not addressed further. 


\subsubsection{Does GA Treatment Affect Monocytes in the Draining Lymph Node?}

In the co-immunisation treatment model, GA may act on blood-derived monocytes that infiltrate the site of immunisation. However, the site of immunisation (subcutaneous at the hind limb flanks) was not accessible to cell recovery techniques used in this study. Hence, analysis was limited to cells that infiltrate the local draining (inguinal) lymph nodes.

In the draining lymph nodes, GA-FITC staining could not be detected on any cells after immunisation with the labelled drug. Staining may have been below detection level, due to dispersion, degradation or slow leakage of GA-FITC from the emulsion. Alternatively, the soluble factor that presumably mediates binding to monocytes in the blood may be absent from the lymph nodes. Finally, cells in the lymph node may be unable to bind GA and cells that may bind GA at the site of immunisation may not subsequently home to the draining lymph node, or loose staining during migration (see figure 4.6). In support of the latter possibility, the percentage of overall CD11b ${ }^{\text {hi }}$ monocytes remained unchanged in the draining lymph nodes following immunisation (see figure 4.11).

Monocyte populations in spleens and the blood also remained constant after immunisation. In contrast, accumulation of $\mathrm{CD} 11 \mathrm{~b}^{\text {hi }} \mathrm{Ly}_{6 \mathrm{G}}^{+}$granulocytes was observed in the spleens and the blood of immunised mice (see figure 4.12). This observation in part contradicts previous findings of Zhu . (133), who observed accumulation of both CD $11 b^{\text {hi }}$ Ly6G $^{+}$granulocytes and CD $11 b^{\text {hi }}$ Ly6G $^{-}$Ly6C ${ }^{\text {hi }}$ inflammatory monocytes in spleens of CFA immunised BALB/c mice. In the study by Zhu , the percentage of monocytes was low $(<0.5 \%)$ in naïve spleens (133), and considerably more monocytes were present in naïve spleens of C57BL/6J mice used in this study ( $>2 \%)$. Significant monocyte accumulation may not have been observed in the present study because of the more pronounced presence of monocytes in the naïve tissues. Alternatively, enrichment of monocytes may have been masked by the concomitant strong granulocyte accumulation.

The lack of monocyte infiltration into the draining lymph nodes after immunisation with CFA is consistent with findings of Zhu (133). Escape of monocyte infiltration from detection due to phenotypic transformation is an unlikely possibility, because monocytes usually retain expression of $\mathrm{CD} 11 \mathrm{~b}$ during heir differentiation into macrophages or DCs $(126,146)$. In addition, adoptively transferred 
monocytes could not be detected in the draining lymph nodes of immunised congenic recipients (see figure 4.13). However, the failure to detect transferred cells may have been due to a general loss of these cells, as CD45.2 $2^{+}$cells could not be recovered from the blood of the recipients, either.

The importance of draining lymph nodes as sites of monocyte-mediated protection in GA treatment remains unclear. Although GA-reactive T cells are present in the draining lymph nodes after immunisation with GA (see figure 3.15), these T cells may become activated by APCs other than monocytes, such as conventional DCs, which do not express CD11b (126). Alternatively, monocytes may act at other sites such as in peripheral tissue or the spleen to contribute to suppression of autoreactivity (80).

\subsubsection{T Cell Suppression by GA-Modulated Monocytes}

Naïve blood monocytes have an intrinsic ability to suppress polyclonal as well as antigen specific $\mathrm{T}$ cell proliferation (see figures 4.7 and 4.8). These monocytes can be classified as MDSCs, because they are of myeloid origin, have an immature phenotype and the ability to suppress $\mathrm{T}$ cell proliferation (135). However, it is a common perception that MDSCs are induced by non-host derived factors, such as tumours, allografts or infectious agents (138-140). As $\mathrm{T}$ cell suppressive ability is an intrinsic feature of the monocytes investigated in this study, they are referred to as suppressive monocytes to differentiate them from elicited MDSCs.

$\mathrm{GA}^{+}$suppressive monocytes are a heterogeneous population of mononuclear $\mathrm{CD} 11 \mathrm{~b}^{\text {hi }} \mathrm{F} 4 / 80^{\text {lo }}$ cells (see figure 4.4). Various phenotypes of mononuclear MDSCs have been described do date, including phenotypes of inflammatory monocytes, DCs and macrophages $(133,140)$. Hence it is not clear which subpopulation of $\mathrm{GA}^{+}$monocytes exhibits $\mathrm{T}$ cell suppressive activity. It is also possible that two or more subpopulations act in concert to achieve T cell suppression.

GA treated monocytes were superior to naïve monocytes in suppressing polyclonal $\mathrm{T}$ cell proliferation, but no differences were observed in the suppression of antigen specific proliferation (see figures 4.7 and 4.8). The lack of difference in the ability of naïve and GA-treated monocytes may have been due to limitations of the available assays for the detection of suppression.

Measurement of $\left[{ }^{3} \mathrm{H}\right]$-thymidine incorporation and measurement of CFSE dilution are both established methods for the assessment of $\mathrm{T}$ cell proliferation (147). However, both methods have their limitations, and one method may be preferable over the other 
depending on the experimental settings (148). Polyclonal stimulation of splenocytes results in a robust proliferative response that can be measured by $\left[{ }^{3} \mathrm{H}\right]$-thymidine incorporation even at low cell numbers (see figure 4.7). In contrast, antigen specific proliferation is less pronounced, and suppression of antigen specific proliferation by $\left[{ }^{3} \mathrm{H}\right]$-thymidine uptake at low cell numbers can be problematic (148). The procedure of monocyte enrichment was accompanied by low yield in cell numbers $\left(2 \times 10^{5}-\right.$ $5 \times 10^{5}$ cells from total blood of five mice), although it consistently produced good purity of mononuclear $\mathrm{CD} 11 \mathrm{~b}{ }^{\text {hi }}$ cells ( $>80 \%$ pure). Due to the low cell numbers, suppression of antigen specific proliferation could not be comprehensively measured by $\left[{ }^{3} \mathrm{H}\right]$-thymidine incorporation.

Measurement of CFSE dilution in responder populations is a more adequate method for studying proliferation at low cell numbers, due to the higher sensitivity of flow cytometric analysis (148). However, detecting subtle differences between two suppressive cell populations by measuring CFSE dilution in responder cells can be difficult. This is particularly the case when the duration of suppression is of interest, because of the prolonged time span of the assay. In contrast, $\left[{ }^{3} \mathrm{H}\right]$-thymidine uptake that is restricted to a defined time span that can be freely chosen (147). Hence, the failure to detect increased suppression by GA treated monocytes as compared to naïve monocytes may have been due to limitations of the assays. Hence, it cannot be conclusively resolved whether GA treatment affects the ability of monocytes to suppress antigen specific $\mathrm{T}$ cell proliferation

Reduced proliferation of $\mathrm{MOG}_{35-55}$ specific $\mathrm{T}$ cells after intravenous GA treatment (see figure 4.9) indicates that differences between GA treated and naïve monocytes may indeed have escaped detection in the antigen specific assay. However, there is no direct evidence that reduced proliferation in GA treated mice is attributable to suppressive monocytes. Demonstrating recovery of $\mathrm{T}$ cell proliferation upon depletion of monocytes would provide direct evidence for suppressive function of monocytes. However, depletion of monocytes using $\mathrm{Cl}_{2} \mathrm{MDP}$ loaded liposomes (144) interferes with T cell proliferation and therefore cannot be used for the in suppression assay (C. Slaney, unpublished data). Hence, currently there is only an indirect correlation between the binding of GA to monocytes after intravenous injection and reduced $\mathrm{MOG}_{35-55}$ specific $\mathrm{T}$ cell proliferation after intravenous GA treatment. 
Reduced proliferation of $\mathrm{T}$ cells was associated with GA treatment both

and , but impaired proliferation of $\mathrm{MOG}_{35-55}$ specific CD4 $\mathrm{T}$ cells was not observed in GA treatment of EAE (see figure 4.15). These findings suggest that direct suppression of CD4 $\mathrm{T}$ cells may not be a central mechanism that contributes to EAE suppression in the co-immunisation GA treatment model. However, suppression of $\mathrm{MOG}_{35-55}$ specific $\mathrm{T}$ cells may have escaped detection in this setting, due to immune manipulations in the EAE model (38).

EAE induction requires injection of CFA, which delivers a strong proinflammatory stimulus to the immune system (38). CFA promotes the induction of robust inflammatory $\mathrm{T}$ cell responses that may have overcome antigen non-specific suppression that can potentially be exerted by GA modulated monocytes. Since CFA is not present in MS patients during GA treatment, it remains possible that the antigen non-specific suppression of T cells observed in this study contributes to GA therapy in MS.

\subsubsection{Targeting GA to Monocytes in EAE Treatment}

Regardless of whether or not they can directly suppress T cells, monocytes are key players in GA treatment of EAE. Monocytes initiate both anti-inflammatory and regulatory $\mathrm{T}$ cell responses upon GA treatment of the murine disease (80). As such monocytes represent a useful target for GA treatment of EAE and therefore of MS.

The cell surface molecule chosen for targeting GA to monocytes was CD11b. Expression of this marker is not limited to $\mathrm{GA}^{+}$cells in the blood, but all $\mathrm{GA}^{+}$cells express CD11b at high levels (see figure 4.4). Moreover, drugs can be targeted efficiently to $\mathrm{Cd} 11 \mathrm{~b}{ }^{\text {hi }}$ macrophages by conjugating them to a monoclonal antibody to CD11b (149), and antigen targeting to $\mathrm{CD} 11 \mathrm{~b}$ results in the efficient delivery of the antigens into the MHCII antigen presentation pathway (150). Hence targeting GA to CD11b was hypothesised to promote GA-specific $\mathrm{T}$ cell responses by enhancing its uptake and MHCII-restricted presentation.

M1/70-GA failed to ameliorate EAE in the co-immunisation treatment model. As the conjugate was administered at an approximately 500-fold less molar amount than the therapeutic dose of free GA, the dose may have been too low to suppress EAE. In addition, expression of CD11b by other cell types like neutrophils (133) may have resulted in the sequestration of M1/70-GA, further reducing the amount of GA that is effectively delivered to monocytes. Alternatively, GA may have been efficiently delivered to monocytes, but failed to induce their differentiation towards an 
immunosuppressive or anti-inflammatory phenotype, which is required for protective immunity $(80,132)$. The latter scenario is supported by previous findings, which showed that immune responses to antigens targeted to $\mathrm{CD} 11 \mathrm{~b}$ expressing cells are biased towards $\mathrm{T}_{\mathrm{H}} 1$, producing high amounts of IFN- $\gamma$, but no IL-4 or IL-10 (150). Hence, targeting GA to $\mathrm{CD} 11 \mathrm{~b}$ may skew the resulting immune response in a way that is not favourable for treatment.

To test the dependency of GA therapy on monocytes in EAE, monocyte-depleted mice were treated with GA. The treatment was as efficacious as in mice that had not received monocyte-depleting treatment. Monocyte depletion by $\mathrm{Cl}_{2} \mathrm{MDP}$-liposomes is only temporary, and the cells are replenished by myeloid progenitors in the bone marrow within three to four days following liposome administration (131). Although monocytes were only depleted in the early phase after co-immunisation, this phase is considered to be of great importance for the initiation of pathogenic as well as protective $\mathrm{T}$ cell responses in EAE (see figure 4.14 and ref. (80)). Hence, GA therapy does not depend on the presence of monocytes in this early phase of the disease.

It is possible that a delayed response to GA by monocytes after their replenishment from bone marrow progenitors following $\mathrm{Cl}_{2} \mathrm{MDP}$ depletion is sufficient for protection from EAE. Monocytes are involved in pathogenic events such as destruction of CNS tissue during later stages of EAE (124); hence longer-term depletion of monocytes by multiple injections of $\mathrm{Cl}_{2} \mathrm{MDP}$ liposomes results in partial resistance to EAE (52). In addition, $\mathrm{Cl}_{2} \mathrm{MDP}$ liposomes have been shown to partially deplete other cell types in the blood (131), as well as phagocytic cells in other tissues $(151,152)$. As a consequence, long-term depletion of monocytes is not a suitable approach to investigate the dependence of GA treatment on monocytes.

\subsubsection{The Role of Trypsinogen and DM SMEZ2}

Trypsinogen and DM SMEZ2 stained a similar cell type in the blood as GA, albeit with different intensity (see figure 4.1). The different intensity may in part be due to the different efficacy of their labelling with the fluorophor. Alexa488 is reactive to primary amines, and can therefore label GA more brightly, due to the high abundance of lysine residues in the copolymer (62). Furthermore, higher molar amounts of GA (7-10 kDa in average) than trypsinogen $(25 \mathrm{kDa})$ and DM SMEZ2 $(24 \mathrm{kDa})$ were injected 
into the mice, which could also contribute to the brighter staining of monocytes from mice injected with GA.

Trypsinogen was found to suppress EAE at a dose of $500 \mu \mathrm{g}$ by co-immunisation treatment (see A1.6). In spite of being an inactive precursor of trypsin in theory, trypsinogen $^{2}$ can have up to $10 \%$ of the enzymatic activity of trypsin ${ }^{3}$ supplied by the same manufacturer. Furthermore, trypsinogen solutions are only stable in a range of $\mathrm{pH} 2-4$, and autocatalytic activation to trypsin occurs in neutral buffers ${ }^{4}$. As a consequence, protection from EAE upon co-immunisation with trypsinogen may be due to degradation of the disease causing peptide, rather than having an effect on monocytes.

A conjugate of DM SMEZ2 and $\mathrm{MOG}_{35-55}$ is able to ameliorate EAE at a dose as low as $2 \mu \mathrm{g}$ when administered in Incomplete Freund's Adjuvant (C. Slaney, unpublished data). Treatment with this DM SMEZ2-MOG ${ }_{35-55}$ conjugate may target the peptide to monocytes, rendering them able to induce antigen-specific anergy or activation and/or expansion of T-regs. Unconjugated DM SMEZ2 was not available in sufficient quantities to evaluate its capacity to suppress EAE at a comparable dose to GA. The functional relevance of the binding of these three compounds to blood monocytes for the suppression of EAE therefore remains unclear.

\subsubsection{Conclusions}

The findings presented in this chapter showed that GA could bind to murine monocytes , indicating that monocytes may be its primary targets in therapy. Intravenous GA treatment enhanced the intrinsic ability of monocytes to suppress $\mathrm{T}$ cell proliferation, suggesting that suppression of myelin-specific $\mathrm{T}$ cells by GA modulated monocytes may contribute to the efficiency of GA therapy. As discussed in section 4.3.3, the current EAE model has inherent inadequacies of an experimental model in mimicking immunopathogenesis of the human disease; hence direct suppression of $\mathrm{T}$ cells may contribute to GA therapy in MS, although impaired T cell proliferation was not detected in EAE in this study.

Monocytes are key players in EAE suppression by GA, and possible targets of the drug during treatment. However, targeting GA to monocytes did not

\footnotetext{
${ }^{2}$ http://www.sigmaaldrich.com/catalog/search/SpecificationSheetPage/SIGMA/T1143

${ }^{3} \mathrm{http}: / /$ www.sigmaaldrich.com/catalog/search/SpecificationSheetPage/SIAL/T1426

${ }^{4} \mathrm{http}: / /$ www.sigmaaldrich.com/sigma/product\%20information\%20sheet/T1143pis.pdf
} 
suppress EAE at low dose. Disease amelioration at higher doses of conjugate remains possible, but the likely scenario is that targeted GA failed to induce the phenotype shift in monocytes that renders them able to prime anti-inflammatory or regulatory $\mathrm{T}$ cell responses. Conjugating GA to antibodies may result in a loss of its immunomodulatory properties.

A crucial dependence of GA treatment on monocytes could not be conclusively demonstrated in this study. Depletion of monocytes with $\mathrm{Cl}_{2} \mathrm{MDP}$ liposomes may not be a suitable approach to investigate the possible dependence of GA on monocytes, as discussed in section 4.3.4. Alternatively, GA may also be able to induce antiinflammatory/tolerogenic DCs or type II activated macrophages that can compensate for the absence of monocytes. GA, trypsinogen and DM SMEZ2 all bound to blood monocytes after intravenous injections. A correlation between binding to blood monocytes of these three compounds and their ability to suppress EAE in different settings is possible, but remains speculative based on the current findings. 


\section{CHAPTER 5:}

\section{GENERAL DISCUSSION}




\subsection{Summary}

GA is currently in use for the treatment of RRMS and can suppress EAE. The primary target of GA are considered to be innate cells, which act as APCs and expand Foxp $3^{+}$T-regs or induce immune deviation by stimulating $\mathrm{T}_{\mathrm{H}} 2$ responses $(80)$. GA-modulated innate cells may also suppress auto-reactive $\mathrm{T}$ cells directly in an antigen non-specific manner (83). However, it is not known which type of innate cell GA preferentially acts on. The objectives of this work were to identify the target cells of GA, and to investigate direct effects of GA on its target cells. In addition, the potential of high-affinity targeting GA directly to cell surface molecules on its target cells to improve EAE treatment was assessed.

Upon intravenous injection, fluorophor-labelled GA was found to bind to blood monocytes independently of their MHCII expression. Intravenous GA treatment enhanced the intrinsic ability of the monocytes to suppress $\mathrm{T}$ cell proliferation

Proliferation of myelin specific CD4 $\mathrm{T}$ cells was also reduced following intravenous GA treatment. However, GA treatment did not reduce $\mathrm{T}$ cell proliferation in EAE.

Direct binding of GA to a distinct cell population is reported for the first time in this study. The identification of monocytes as the probable target of GA is of great value, because it suggests that focusing on monocytes may pave the way for improved GA therapy or the development of alternative immunotherapies for MS. In addition, it is shown for the first time within this study that direct suppression of myelinspecific $\mathrm{T}$ cells by GA modulated monocytes may be part of the mechanism by which GA inhibits autoimmune responses in EAE and MS.

Targeting GA to monocytes with an anti-CD11b antibody or to the sum of APCs with an anti-MHCII antibody did not ameliorate EAE. MCHII targeted GA failed to suppress EAE in spite of substantially enhanced $\quad \mathrm{T}$ cell proliferation in response to MCHII targeted antigens, suggesting that enhanced proliferation of GA specific T cells alone is not sufficient for protection from EAE. As a conjugate GA may fail to alter monocyte function, and conjugate-treated monocytes/APCs may thus not be able to exhibit immunosuppression or to prime regulatory/suppressive $\mathrm{T}$ cell responses. 


\subsection{Antigen Targeting Strategies in Disease Treatment}

Professional APCs can present antigen on MHCII or MHCI molecules to induce CD4 or CD8 T cell responses, respectively (6). Among the APCs, DCs are the most potent inducers of CD4 and CD8 T cell responses, owing to the high expression of cell surface receptors and co-stimulatory molecules involved in these processes. DCs also have the greatest capacity for cross-presentation, but other subsets of APCs are also capable of directing extracellular antigens into the cross-presentation pathway (6).

Antigen and drug targeting by the use of monoclonal antibodies has become a critical part of the treatment of cancer and infectious diseases as well as vaccine development (99). These strategies are based on the ability of the antibodies to specifically bind to the biological target of choice in the patient or the experimental host. Targets are usually pathogens, malignant cells in tumours or APCs for vaccinations (99). Even though APCs possess mechanisms of internalising and processing antigens for their subsequent presentation, this process can be made by far more efficient by targeting antigens directly to cell surface molecules of these cells (99).

\subsubsection{MHCII as an Antigen Target}

Cell surface molecules on APCs that have been used for targeting antigens include MHCII (120), the C-type lectin receptors DEC205 (153) and the mannose receptor CD206 (99); Fcy receptors (154) and complement receptors (155). Antigen targeting studies usually aim at the initiation or enhancement of immunity towards the targeted antigen. MHCII is a particularly attractive candidate for this purpose, since it is regularly internalised and recycled within endocytic compartments (94). In this way, MHCII-targeted antigens are directly delivered into the antigen presentation pathway.

In this study, significantly enhanced CD4 and CD8 $\mathrm{T}$ cell responses were observed when antigens were targeted to MHCII (see figures 3.10 and 3.13). The use of engineered MHCII specific antibodies that contain CD4 T cell epitopes has been reported to result in a similar increase of CD4 T cell proliferation (115). Furthermore, enhanced cross-presentation upon antigen targeting to MHCII has also been reported (117).

Cross-presentation of free antigens is strongly biased towards the presentation of high-abundance antigens (95). The knowledge that antigens can be efficiently targeted to promote cross-presentation should therefore be particularly useful in the design of 
vaccines against intracellular pathogens and tumors where robust CD8 T cell responses to low antigen doses are desirable (156). In addition, simultaneous stimulation of CD4 T cells has the potential to boost the CTL response and further enhance the potency of the targeted vaccines (157). Further investigation of CD8 T cell responses to MHCII targeted antigens was beyond the scope of this study, but M5/114-OVA is currently being tested as an anti-tumour vaccine in a murine melanoma model (E. Spittle, unpublished data).

\subsubsection{Does Targeting GA to Cell Surface Molecules Have Therapeutic Potential?}

GA specific anti-inflammatory CD4 $\mathrm{T}$ cells are present in treated mice and MS patients $(80,158)$, and GA has been shown to bind to MHCII molecules Hence MHCII molecules on the surface of APCs are generally considered to be the primary target of GA. In addition, GA treatment did not suppress EAE in $\mathrm{MOG}_{35-55} \mathrm{TCR}$ transgenic mice in this study (see figure 3.8), suggesting that expansion of GA specific CD4 $\mathrm{T}$ cells may be of crucial importance for treatment. Therefore MHCII was an attractive cell surface molecule for specific targeting of GA into the antigen presentation pathway, in order to achieve a reduction of the therapeutic dose.

Targeting antigens to MHCII in absence of adjuvant results in increased uptake and presentation of antigen and the initiation of robust humoral immunity to a secondary challenge with the same antigen (155). This response depends on CD4 T cell help, suggesting the preferential induction of $\mathrm{T}_{\mathrm{H}} 2$ responses by the targeted antigen, that can subsequently promote humoral immunity (119). However, CFA is a strong proinflammatory adjuvant (38), and its presence may substantially influence the nature of immune responses. Free GA can suppress EAE despite the $\mathrm{T}_{\mathrm{H}} 1$ promoting effects of CFA (see figure 3.3), but the M5/114-GA conjugate may be incapable of acting against the pro-inflammatory effects of the adjuvant. Alternatively, MHCII targeted GA may be sequestered by $\mathrm{MHCII}^{\mathrm{hi}}$ cells that are irrelevant to disease suppression, thus making GA unavailable to its real targets.

It is not known if GA preferentially acts on a particular type of $\mathrm{MHCII}^{+}$cell

In addition, recent findings have demonstrated the induction of type II monocytes by GA in an MHCII independent manner (80). Hence, although MHCII-restricted presentation of GA epitopes is recognised to be important for the activation of antiinflammatory or regulatory $\mathrm{T}$ cells, the role of MHCII during the preceding modulation of APCs is rather questionable (86). The results of this study support this view, as it was 
found that the majority of $\mathrm{GA}^{+}$monocytes in the blood did not express MHCII (see figure 4.4), and GA staining occurred in an MHCII independent manner (see figure 4.5).

The binding assays suggest that monocytes may be the preferred target of GA (see figure 4.1). However, targeting GA to $\mathrm{CD}_{1} 1 \mathrm{~b}^{+}$cells did not ameliorate EAE at low dose (see figure 4.18). Inadequate dosage or sequestration of the targeted drug by irrelevant cells (such as neutrophils that also express CD11b) are possible explanations for the failure of M1/70-GA to suppress EAE. However, the most likely scenario is that as a conjugate, M1/70-GA failed to exhibit immunomodulation on its target cells. Targeting antigens to CD11b can enhance proliferative responses (150), but increased antigen presentation alone is not sufficient for protection from EAE in absence of APCderived anti-inflammatory mediators (80). In addition, targeting antigens to CD11b results in a $\mathrm{T}_{\mathrm{H}} 1$ biased immune response (150), which may further counteract the suppressive potential of GA.

CD11b and MHCII are two candidate cell surface molecules that were evaluated as targets for GA in this study, but amelioration of EAE was not observed using either of these target molecules (see figures 3.16 and 4.18). The most plausible explanation for the failure of GA targeting is that when conjugated, the immunomodulatory effect of GA on its target cells is lost. However, these findings do not imply that further attempts to target GA should be abandoned, but rather suggest that the choice of the cell surface target molecule is of crucial importance to elicit the appropriate immune response.

The type of immune response that is initiated by targeted antigens greatly depends on the choice of the cell surface target (99). For example, targeting antigens to CD206 results in MHCI-restricted antigen presentation and potent CD8 T cell mediated immunity (99). In contrast, targeting antigens to DEC205, a related cell surface receptor, results in antigen specific hyporesponsiveness of both CD8 and CD4 T cells $(153,159)$. The different outcomes for targeting CD206 and DEC205 are likely due to differential localisation of the two receptors after their internalisation (99). This example illustrates how subtle functional differences between cell surface targets can influence the immune response to the targeted antigen.

Anti-inflammatory $\mathrm{T}_{\mathrm{H}} 2$ responses can be induced by targeting OVA protein to FcyRs (154). Immune complexes comprised of OVA and OVA-specific antibodies are taken up by macrophages through Fc $\gamma$ Rs. The macrophages then present OVA-derived peptides to CD4 T cells, which are by this way stimulated to produce IL-4 and IL-10, but 
only low amounts of IFN- $\gamma$ and IL-12 (154). The $\mathrm{T}_{\mathrm{H}} 2$ biased response is observed even when macrophages are pre-activated with IFN- $\gamma$ prior to their exposure to the immune complexes. In contrast, $\mathrm{T}$ cells that are activated by macrophages exposed to free OVA produce high amounts of IFN- $\gamma$ and IL-12, but low amounts of IL-4 (154).

Targeting antigens to FcyRs using anti-FcyR-antigen conjugates may be as efficient in biasing $\mathrm{T}$ cell differentiation as the use of immune complexes. The above mentioned immune complexes consist of OVA protein opsonised by a 10-fold molar excess of Ig (154). These immune complexes may cross-link Fc $\gamma$ Rs, and cross-linking may also occur with antigens that are chemically conjugated to a specific antibody, as the reaction conditions during conjugation can be modified in favour of high molecular weight complexes that contain several antibody molecules interconnected by proteins or copolymers (see section 3.2.2.1 and figure 3.9).

It is not known whether GA is taken up by APCs through an active or passive mechanism. Experimental evidence on any cell surface molecules that are possibly involved in this process is currently lacking as well (86). The mechanism by which GA induces differentiation of anti-inflammatory/type II APCs also remains to be unveiled. A better understanding of this process is necessary to ensure the appropriate choice of cell surface molecules in further approaches of targeting. The choice of a cell surface molecule that can be functionally involved in acquisition of the desired anti-inflammatory APC phenotype may prove efficient in suppression of self-reactive $\mathrm{T}$ cell responses, and hence be a plausible strategy to make GA therapy more efficient.

\subsection{Glatiramer Acetate: Update on Mechanism of Action}

\subsubsection{How Important is Direct Binding to MHCII?}

The view that GA acts as an altered peptide ligand by blocking MHCII molecules and TCR antagonism to myelin-derived peptides $(62,89)$ has predominated for a long time. In addition, MHCII restricted presentation of GA epitopes to CD4 T cells and induction of anti-inflammatory $\mathrm{T}$ cells is considered to be an important part of GA therapy (63). However, recent findings have demonstrated that during GA treatment, monocytes acquire an anti-inflammatory type II phenotype independently of MHCII expression (80), suggesting that GA can modulate APCs in an MHCII independent 
manner. The findings presented in this study provide additional evidence of MHCII independent effects of GA on monocytes.

Binding of fluorophor-labelled GA to MHCII expressing cells was not observed , and binding to monocytes was independent of MHCII $\quad$ (see figures 4.1

and 4.3). While the lack of binding contradicts earlier findings (87) and may be attributable to differences in the experimental settings, binding of GA to any cell type or cell surface molecule has not been reported. It should be noted that, binding assays were performed by intravenous injection of fluorophor-labelled GA, and intravenous treatment failed to suppress EAE. However, intravenous binding assays better represent clinical GA therapy than binding assays using purified cell populations and high concentration of GA $(87,88)$. Hence, the results presented here further challenge the hypothesis that direct binding of GA to MHCII and TCR antagonism to myelin antigens is an important part of the mechanism of GA.

\subsubsection{Divergent T cell Mediated Mechanisms of Suppression}

Induction of $\mathrm{T}_{\mathrm{H}} 2$ cells after GA treatment is well-established in EAE and MS (6870). However, GA can efficiently suppress EAE in mouse strains that lack the key $T_{H} 2$ cytokines IL-4 and IL-10 (81). This finding shows that, even though GA-induced $\mathrm{T}_{\mathrm{H}} 2$ cells can counteract pathogenic cells $(68,74)$, this process is not essential for EAE suppression.

GA can also induce Foxp $3^{+}$T-regs that contribute to suppression of EAE (77) and MS (79). Systemic depletion of $\mathrm{CD} 25^{+}$cells by administration of a monoclonal antibody (clone PC61) before EAE induction resulted in substantial loss of protection by GA (see A1.7). However the loss of protection was not absolute, since GA-treated mice lacking $\mathrm{CD} 25^{+}$cells developed less severe disease than untreated mice lacking the same cells, in agreement with a recent report (82). Thus the loss of Foxp3 $3^{+}$T-regs does not completely abrogate EAE suppression by GA.

GA induces both IL-4 producing $\mathrm{T}_{\mathrm{H}} 2$ cells and Foxp ${ }^{+}$T-regs in mice, and these two populations do not overlap (80). Induction of these two $\mathrm{T}$ cell populations has also been independently observed in GA treated MS patients $(69,160)$. Hence, GA induced $\mathrm{T}_{\mathrm{H}} 2$ and T-regs may act in an additive manner, but independently of each other to control or suppress pathogenic T cells. Furthermore, T-regs may have the ability to compensate for the loss of the $\mathrm{T}_{\mathrm{H}} 2$ effector arm, whereas in the opposite scenario, $\mathrm{T}_{\mathrm{H}} 2$ cells seem to be less capable of compensating for the loss of T-regs $(81,82)$. 


\subsubsection{Possible Contribution of Multiple APC Subsets to GA Therapy}

Despite the prominent $\mathrm{T}$ cell mediated protective effects that are associated with treatment, GA does not affect $\mathrm{T}$ cells directly (84). The primary targets of the drug are considered to be cells of the innate immune system that can act as APCs (86). GA can act on a range of cells with the potential to present antigen to CD4 T cells , including DCs $(85,161)$, monocytes $(162,163)$, macrophages (125) and microglia (84).

The significance of each individual APC subset remains poorly established . GA can act through monocytes to suppress EAE (80), and modulation of monocytes in GA treated MS patients has also been observed (84). However, in this study GA was found to be as efficient in suppressing EAE when monocytes were temporarily depleted (see figure 4.16). Because monocytes are replenished within three to four days by the bone marrow after their depletion (131), it is possible that a delayed monocyte-mediated response following reconstitution of blood monocytes is sufficient for EAE suppression.

Alternatively, GA may be able to act on multiple subsets of innate cells that have the capacity of antigen presentation, such as monocytes, DCs and macrophages. Moreover, some types of APCs may be able to compensate for the loss or malfunction of others. For example, GA modulated DCs may induce protective T cell responses in the absence of monocytes. Consistent with this scenario, some of the CD11 b ${ }^{\text {hi }}$ cells that bind GA in the blood also expressed CD11c (see figure 4.4), suggesting that they could be monocyte-derived DC precursors (126). Hence it is possible that GA can also bind to and act through DCs. The absence of mature DCs from the blood provides a possible explanation for the lack of GA staining of cells that show the cell surface phenotype of CD11 $\mathrm{c}^{\mathrm{hi}} \mathrm{MHCII}^{\mathrm{hi}}$ DCs. Further studies are required to establish the significance of each particular APC subset in the treatment of EAE and MS.

\subsubsection{Possible Role for Suppressive Monocytes in GA Treatment}

Monocytes and macrophages are generally associated with pathogenic events such as the breakdown of the BBB and destruction of CNS tissue in EAE and MS (52). However, GA can alter these cells and , rendering them capable of inducing protective $\mathrm{T}$ cell responses $(80,125)$.

Recent findings have raised the possibility that $\mathrm{T}$ cell independent mechanisms may contribute to GA's therapeutic effect in EAE (114). Furthermore, CFA-induced accumulation of monocytes that inhibit $\mathrm{T}$ cell proliferation through the production of NO 
has been observed in $\operatorname{EAE}(131,133)$. The results presented here show for the first time that GA treatment can induce antigen non-specific suppression of $\mathrm{T}$ cell proliferation through modulation of monocytes (see figure 4.7). Hence, CFA and GA can induce suppressive monocytes independently of each other. Similar to CFA-induced monocytes, GA-induced monocytes may also suppress $\mathrm{T}$ cells through the production of NO.

GA treatment has been shown to induce type II monocytes (80), and NO production is one of the characteristics of type II activation (132). Hence NO is a possible mediator of GA-induced T cell suppression by monocytes. However, the role of NO in autoimmune disorders is complex. Mice that lack iNOS develop more severe EAE than their wild-type counterparts (45). In contrast, treatment of mice with a selective inhibitor of iNOS ameliorates EAE (164), suggesting that NO plays multiple roles in the development and prevention of CNS autoimmunity.

High levels of NO produced by infiltrating macrophages are associated with tissue damage in the CNS and the breakdown of the BBB (52). In contrast, NO production in the periphery is associated with immunosuppressive effects such as induction of lymphocyte apoptosis (136). In addition, splenocytes from iNOS deficient mice show increased proliferation in response to $\mathrm{MOG}_{35-55}$, with a negative correlation between NO levels and T cell proliferation in these cultures (45).

GA has been observed to promote lymphocyte apoptosis in MS patients (113), implicating a possible role for suppressive monocytes in controlling $\mathrm{T}$ cell proliferation in the periphery. Although GA treatment correlates with an overall reduction of total free radicals in MS patients (165), the specific effect on individual free radical species, and NO in particular, is unknown. Hence, the mechanism of enhanced T cell suppression may involve a GA-induced enhancement of NO production by monocytes.

Antigen non-specific $\mathrm{T}$ cell suppression by monocytes may contribute to GA therapy, but the significance of this suppression remains to be established in EAE models. In the co-immunisation model, suppression may have been overcome or masked by the presence of a strong inflammatory adjuvant that promotes $\mathrm{T}$ cell proliferation (38). In addition, $\mathrm{T}$ cell suppression by monocytes is transient in nature (C. Slaney, unpublished data), suggesting that suppression may also be transitory and therefore escaped detection due to sustained $\mathrm{T}$ cell proliferation in the EAE model (82). It therefore remains possible that $\mathrm{T}$ cell suppression can be observed in other EAE models or in MS patients who receive daily GA treatment. 


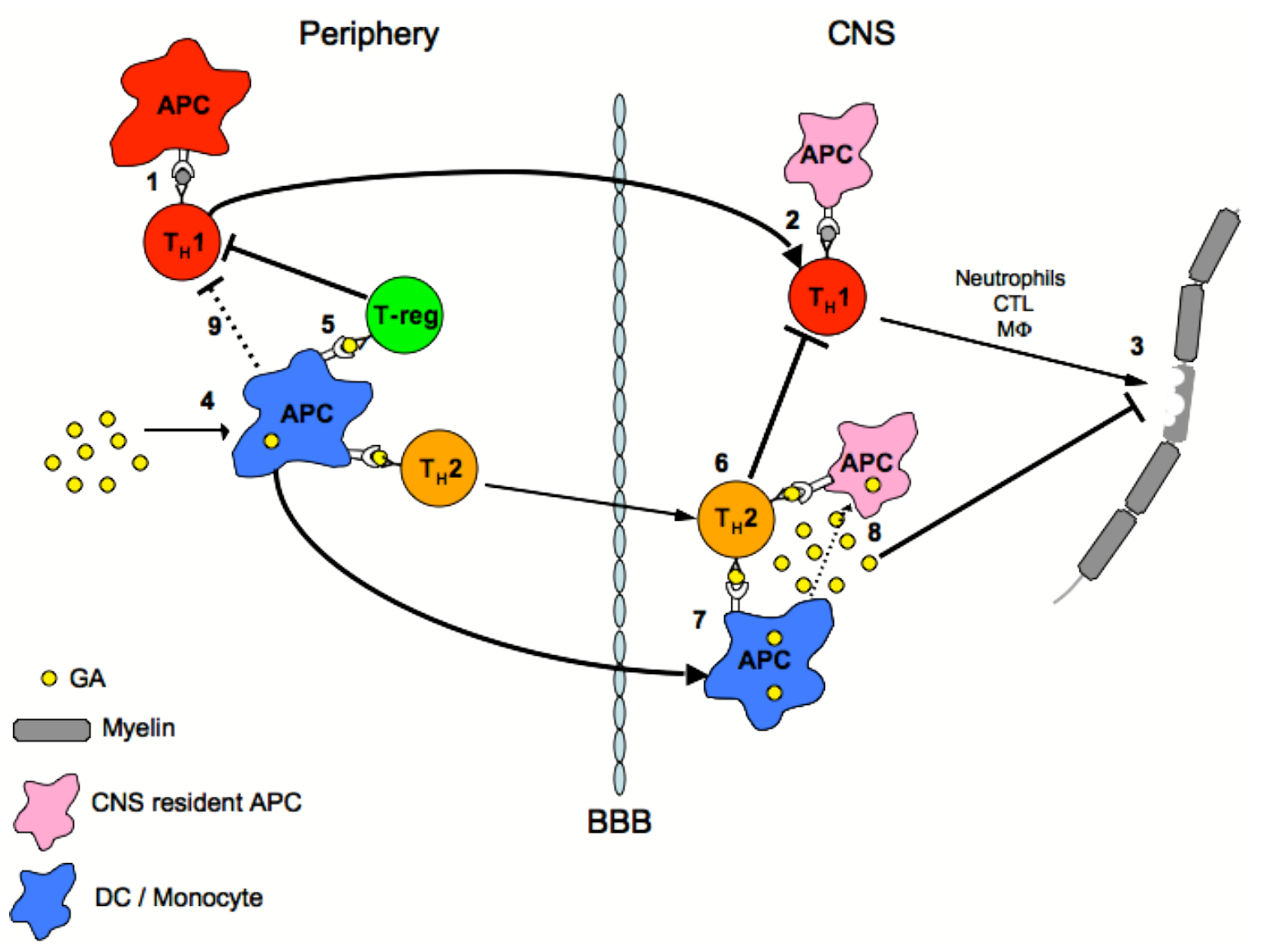

Figure 5.1: Proposed updates on the mechanism of action of GA in EAE.

1: Pathogenic $T_{H} 1$ responses are initiated by the presentation of myelin-derived peptides by activated APCs. 2: The activated $\mathrm{T}_{\mathrm{H}} 1$ cells infiltrate the CNS, where they can be reactivated by myelin peptides presented by local APCs and create an inflammatory environment. 3: As a consequence, other inflammatory cell types are recruited to the CNS that promote demyelination and axonal damage. 4: GA is taken up by APCs in the periphery. It is possible that GA is able to act on multiple subsets of APCs such as monocytes, DCs and macrophages. 5: Upon modulation by GA, these APCs can then induce the expansion of T-regs, which can suppress proliferation of pathogenic $\mathrm{T}$ cells. 6: The APCs also stimulate $T_{H} 2$ cells that are then able to cross the BBB. 7: The $T_{H} 2$ cells can be reactivated by co-migrated or local APCs presenting GA-derived peptides. The activated $\mathrm{T}_{\mathrm{H}} 2$ cells counteract $\mathrm{T}_{\mathrm{H}} 1$ cell-mediated tissue damage by the production of anti-inflammatory and neuroprotective factors. 8: In addition, some DCs may have the ability to release active GA into the CNS environment, which may have direct protective effects on neurons or modulate CNS resident cells. 9: Monocyte or DC-mediated antigen non-specific suppression of pathogenic T cells may also occur in the periphery.

In summary, monocytes, possibly along with other types of APCs, can contribute to GA treatment in several ways (figure 5.1). Induction of $\mathrm{T}_{\mathrm{H}} 2$ cells and T-regs by GA- 
modulated monocytes has recently been demonstrated in EAE (80). The results presented here suggest that GA-modulated monocytes may also suppress pathogenic $\mathrm{T}$ cell responses in an antigen non-specific manner. However, the significance of this antigen non-specific suppression remains to be established in EAE or GA-treated MS patients.

\subsection{Conclusions and Outlook}

GA is a versatile drug that affects the immune system in a plethora of ways. Most prominently, GA-modulated APCs are able to expand Foxp3 ${ }^{+}$T-regs that can suppress pathogenic T cells. Moreover, GA can modulate APCs to prime $\mathrm{T}_{\mathrm{H}} 2$ responses. These $\mathrm{T}_{\mathrm{H}} 2$ responses counteract pro-inflammatory cells in secondary lymphoid organs or in the CNS, and diminish local inflammation. However, the mechanism by which GA modulates APCs remains unclear. The significance of each particular APC subset also remains to be established.

The results presented here show that GA binds to CD1 $1 b^{\text {hi }} F 4 / 80^{\text {lo }}$ monocytes and enhances the intrinsic capacity of these monocytes to suppress $\mathrm{T}$ cell proliferation in an antigen non-specific manner. This study shows for the first time that GA can modify monocytes to suppress the proliferation of myelin-reactive $\mathrm{T}$ cells (figure 5.1; \#9). This antigen non-specific suppression does not appear to be important in the co-immunisation model of EAE treatment but it is possible that direct suppression of $\mathrm{T}$ cells by monocytes contributes to GA treatment in other EAE models and MS patients.

The findings of this study and others also suggest that GA can act through multiple divergent mechanisms. GA has the potential to act on different types of APCs, which can in turn prime at least two different effector functions of the adaptive immune system ( $\mathrm{T}_{\mathrm{H}} 2$ and $\mathrm{T}$-reg). Both of these contribute to suppression self-reactivity and disease amelioration, but they can also act independently of each other.

Further research is necessary to investigate the mechanism by which GA alters APC function. Better understanding of the mechanism of APC modulation may reveal suitable candidate cell surface molecules for targeting GA. Knowledge of the precise mechanism of action of GA, including $\mathrm{T}$ cell dependent and independent processes, will also pave the way for the development of superior immunomodulatory agents for the treatment of MS and other autoimmune disorders. 


\section{REFERENCES}

1. Janeway, C. A., Jr., and R. Medzhitov. 2002. Innate immune recognition.

2. Akira, S., and K. Takeda. 2004. Toll-like receptor signalling.

3. Gordon, S., and P. R. Taylor. 2005. Monocyte and macrophage heterogeneity.

4. Nathan, C. 2006. Neutrophils and immunity: challenges and opportunities.

5. Barrington, R., M. Zhang, M. Fischer, and M. C. Carroll. 2001. The role of complement in inflammation and adaptive immunity.

6. Vyas, J. M., A. G. Van der Veen, and H. L. Ploegh. 2008. The known unknowns of antigen processing and presentation.

7. Calame, K. L. 2001. Plasma cells: finding new light at the end of B cell development.

8. Barry, M., and R. C. Bleackley. 2002. Cytotoxic T lymphocytes: all roads lead to death.

9. Abbas, A. K., K. M. Murphy, and A. Sher. 1996. Functional diversity of helper T lymphocytes.

10. Hogquist, K. A., T. A. Baldwin, and S. C. Jameson. 2005. Central tolerance: learning self-control in the thymus.

11. Jenkins, M. K., A. Khoruts, E. Ingulli, D. L. Mueller, S. J. McSorley, R. L. Reinhardt, A. Itano, and K. A. Pape. 2001. In vivo activation of antigen-specific CD4 T cells.

12. Mackay, C. R. 2001. Chemokines: immunology's high impact factors.

13. Germain, R. N. 1994. MHC-dependent antigen processing and peptide presentation: providing ligands for $\mathrm{T}$ lymphocyte activation.

14. Hennecke, J., and D. C. Wiley. 2001. T cell receptor-MHC interactions up close.

15. Friedl, P., and E. B. Brocker. 2002. TCR triggering on the move: diversity of Tcell interactions with antigen-presenting cells.

16. Pitcher, L. A., and N. S. van Oers. 2003. T-cell receptor signal transmission: who gives an ITAM?

17. Murphy, K. M., and S. L. Reiner. 2002. The lineage decisions of helper T cells.

18. Mosmann, T. R., H. Cherwinski, M. W. Bond, M. A. Giedlin, and R. L. Coffman. 1986. Two types of murine helper T cell clone. I. Definition according to profiles of lymphokine activities and secreted proteins.

19. Bettelli, E., T. Korn, M. Oukka, and V. K. Kuchroo. 2008. Induction and effector functions of $\mathrm{T}(\mathrm{H}) 17$ cells.

20. Agnello, D., C. S. Lankford, J. Bream, A. Morinobu, M. Gadina, J. J. O'Shea, and D. M. Frucht. 2003. Cytokines and transcription factors that regulate T helper cell differentiation: new players and new insights.

21. Stockinger, B., and M. Veldhoen. 2007. Differentiation and function of Th17 T cells. 
22. Kyewski, B., and L. Klein. 2006. A central role for central tolerance.

23. Lechler, R., J. G. Chai, F. Marelli-Berg, and G. Lombardi. 2001. T-cell anergy and peripheral $\mathrm{T}$-cell tolerance.

24. Wekerle, H. 2006. Breaking Ignorance: The Case of the Brain. In , Vol. 305. Springer, Berlin and

Heidelberg, p. 25.

25. McQualter, J. L., and C. C. Bernard. 2007. Multiple sclerosis: a battle between destruction and repair.

26. Vignali, D. A., L. W. Collison, and C. J. Workman. 2008. How regulatory T cells work.

27. Tang, Q., and J. A. Bluestone. 2008. The Foxp3+ regulatory T cell: a jack of all trades, master of regulation.

28. Hafler, D. A., J. M. Slavik, D. E. Anderson, K. C. O'Connor, P. De Jager, and C. Baecher-Allan. 2005. Multiple sclerosis.

29. D'Cruz, D. P., M. A. Khamashta, and G. R. Hughes. 2007. Systemic lupus erythematosus.

30. King, C., and N. Sarvetnick. 1997. Organ-specific autoimmunity.

31. Bar-Or, A., E. M. Oliveira, D. E. Anderson, and D. A. Hafler. 1999. Molecular pathogenesis of multiple sclerosis.

32. Noseworthy, J. H., C. Lucchinetti, M. Rodriguez, and B. G. Weinshenker. 2000. Multiple sclerosis.

33. Hafler, D. A. 2004. Multiple sclerosis.

34. Chancellor, A. M., M. Addidle, and K. Dawson. 2003. Multiple sclerosis is more prevalent in northern New Zealand than previously reported.

35. Keegan, B. M., and J. H. Noseworthy. 2002. Multiple sclerosis.

36. Baxter, A. G. 2007. The origin and application of experimental autoimmune encephalomyelitis.

37. Teixeira, S. V., AA; Bolonheis, SM; Muscara, MN. 2005. Experimental autoimmune encephalomyelitis: A heterogenous group of animal models to study human multiple sclerosis.

38. Steinman, L., and S. S. Zamvil. 2005. Virtues and pitfalls of EAE for the development of therapies for multiple sclerosis.

39. Ben-Nun, A., I. Mendel, R. Bakimer, M. Fridkis-Hareli, D. Teitelbaum, R. Arnon, M. Sela, and N. Kerlero de Rosbo. 1996. The autoimmune reactivity to myelin oligodendrocyte glycoprotein (MOG) in multiple sclerosis is potentially pathogenic: effect of copolymer 1 on MOG-induced disease.

40. Hedegaard, C. J., M. Krakauer, K. Bendtzen, H. Lund, F. Sellebjerg, and C. H. Nielsen. 2008. T helper cell type 1 (Th1), Th2 and Th17 responses to myelin basic protein and disease activity in multiple sclerosis.

41. Edan, G., S. Morrissey, and E. Le Page. 2004. Rationale for the use of mitoxantrone in multiple sclerosis.

42. Arnon, R. 1996. The development of Cop 1 (Copaxone), an innovative drug for the treatment of multiple sclerosis: personal reflections.

43. Aranami, T., and T. Yamamura. 2008. Th17 Cells and autoimmune encephalomyelitis (EAE/MS). 
44. Komiyama, Y., S. Nakae, T. Matsuki, A. Nambu, H. Ishigame, S. Kakuta, K. Sudo, and Y. Iwakura. 2006. IL-17 plays an important role in the development of experimental autoimmune encephalomyelitis.

45. Xiao, B. G., C. G. Ma, L. Y. Xu, H. Link, and C. Z. Lu. 2008. IL-12/IFNgamma/NO axis plays critical role in development of Th1-mediated experimental autoimmune encephalomyelitis.

46. O'Connor, R. A., C. T. Prendergast, C. A. Sabatos, C. W. Lau, M. D. Leech, D. C. Wraith, and S. M. Anderton. 2008. Cutting edge: Th1 cells facilitate the entry of Th17 cells to the central nervous system during experimental autoimmune encephalomyelitis.

47. Stromnes, I. M., L. M. Cerretti, D. Liggitt, R. A. Harris, and J. M. Goverman. 2008. Differential regulation of central nervous system autoimmunity by $\mathrm{T}(\mathrm{H}) 1$ and $\mathrm{T}(\mathrm{H}) 17$ cells.

48. Bechmann, I., I. Galea, and V. H. Perry. 2007. What is the blood-brain barrier (not)?

49. Cayrol, R., K. Wosik, J. L. Berard, A. Dodelet-Devillers, I. Ifergan, H. Kebir, A. S. Haqqani, K. Kreymborg, S. Krug, R. Moumdjian, A. Bouthillier, B. Becher, N. Arbour, S. David, D. Stanimirovic, and A. Prat. 2008. Activated leukocyte cell adhesion molecule promotes leukocyte trafficking into the central nervous system.

50. El Behi, M., S. Dubucquoi, D. Lefranc, H. Zephir, J. De Seze, P. Vermersch, and L. Prin. 2005. New insights into cell responses involved in experimental autoimmune encephalomyelitis and multiple sclerosis.

51. McFarland, H. F., and R. Martin. 2007. Multiple sclerosis: a complicated picture of autoimmunity.

52. Benveniste, E. N. 1997. Role of macrophages/microglia in multiple sclerosis and experimental allergic encephalomyelitis.

53. O'Connor, R. A., and S. M. Anderton. 2008. Foxp3+ regulatory T cells in the control of experimental CNS autoimmune disease.

54. Bynoe, M. S., P. Bonorino, and C. Viret. 2007. Control of experimental autoimmune encephalomyelitis by CD4+ suppressor T cells: peripheral versus in situ immunoregulation.

55. Gold, R. 2008. Combination therapies in multiple sclerosis.

56. Frohman, E. M., A. Shah, E. Eggenberger, L. Metz, R. Zivadinov, and O. Stuve. 2007. Corticosteroids for multiple sclerosis: I. Application for treating exacerbations.

57. Steinman, L. 2005. Blocking adhesion molecules as therapy for multiple sclerosis: natalizumab.

58. Hartung, H. P. 2009. New cases of progressive multifocal leukoencephalopathy after treatment with natalizumab.

59. Neuhaus, O., B. C. Kieseier, and H. P. Hartung. 2004. Mechanisms of mitoxantrone in multiple sclerosis--what is known?

60. Jarius, S., and R. Hohlfeld. 2004. [Interferon therapy of multiple sclerosis. Synopsis of various dosage forms].

61. Sorensen, P. S., C. Ross, K. M. Clemmesen, K. Bendtzen, J. L. Frederiksen, K. Jensen, O. Kristensen, T. Petersen, S. Rasmussen, M. Ravnborg, E. Stenager, and N. Koch-Henriksen. 2003. Clinical importance of neutralising antibodies against interferon beta in patients with relapsing-remitting multiple sclerosis. 
62. Neuhaus, O., C. Farina, H. Wekerle, and R. Hohlfeld. 2001. Mechanisms of action of glatiramer acetate in multiple sclerosis.

63. Schrempf, W., and T. Ziemssen. 2007. Glatiramer acetate: Mechanisms of action in multiple sclerosis.

64. Miller, A., V. Spada, D. Beerkircher, and R. R. Kreitman. 2008. Long-term (up to 22 years), open-label, compassionate-use study of glatiramer acetate in relapsingremitting multiple sclerosis.

65. Ziemssen, T., O. Neuhaus, and R. Hohlfeld. 2001. Risk-benefit assessment of glatiramer acetate in multiple sclerosis.

66. Harde, V., and T. Schwarz. 2007. Embolia cutis medicamentosa following subcutaneous injection of glatiramer acetate.

67. Neumann, H., A. Csepregi, M. Sailer, and P. Malfertheiner. 2007. Glatiramer acetate induced acute exacerbation of autoimmune hepatitis in a patient with multiple sclerosis.

68. Aharoni, R., D. Teitelbaum, M. Sela, and R. Arnon. 1997. Copolymer 1 induces T cells of the $\mathrm{T}$ helper type 2 that crossreact with myelin basic protein and suppress experimental autoimmune encephalomyelitis.

69. Neuhaus, O., C. Farina, A. Yassouridis, H. Wiendl, F. Then Bergh, T. Dose, H. Wekerle, and R. Hohlfeld. 2000. Multiple sclerosis: comparison of copolymer-1reactive $\mathrm{T}$ cell lines from treated and untreated subjects reveals cytokine shift from $\mathrm{T}$ helper 1 to $\mathrm{T}$ helper 2 cells.

70. Duda, P. W., M. C. Schmied, S. L. Cook, J. I. Krieger, and D. A. Hafler. 2000. Glatiramer acetate (Copaxone) induces degenerate, Th2-polarized immune responses in patients with multiple sclerosis.

71. Aharoni, R., D. Teitelbaum, O. Leitner, A. Meshorer, M. Sela, and R. Arnon. 2000. Specific Th2 cells accumulate in the central nervous system of mice protected against experimental autoimmune encephalomyelitis by copolymer 1 .

72. Arnon, R., and R. Aharoni. 2004. Mechanism of action of glatiramer acetate in multiple sclerosis and its potential for the development of new applications.

73. Aharoni, R., D. Teitelbaum, M. Sela, and R. Arnon. 1998. Bystander suppression of experimental autoimmune encephalomyelitis by $\mathrm{T}$ cell lines and clones of the Th2 type induced by copolymer 1 .

74. Aharoni, R., B. Kayhan, R. Eilam, M. Sela, and R. Arnon. 2003. Glatiramer acetate-specific $\mathrm{T}$ cells in the brain express $\mathrm{T}$ helper $2 / 3$ cytokines and brainderived neurotrophic factor in situ.

75. Ziemssen, T., T. Kumpfel, H. Schneider, W. E. Klinkert, O. Neuhaus, and R. Hohlfeld. 2005. Secretion of brain-derived neurotrophic factor by glatiramer acetate-reactive T-helper cell lines: Implications for multiple sclerosis therapy.

76. Arnon, R. 2007. Does glatiramer acetate induce neurogenesis in experimental autoimmune encephalomyelitis?

77. Jee, Y., W. H. Piao, R. Liu, X. F. Bai, S. Rhodes, R. Rodebaugh, D. I. Campagnolo, F. D. Shi, and T. L. Vollmer. 2007. CD4(+)CD25(+) regulatory T cells contribute to the therapeutic effects of glatiramer acetate in experimental autoimmune encephalomyelitis. 
78. Hong, J., N. Li, X. Zhang, B. Zheng, and J. Z. Zhang. 2005. Induction of CD4+CD25+ regulatory $T$ cells by copolymer-I through activation of transcription factor Foxp3.

79. Putheti, P., M. Soderstrom, H. Link, and Y. M. Huang. 2003. Effect of glatiramer acetate (Copaxone) on CD4+CD25high T regulatory cells and their IL-10 production in multiple sclerosis.

80. Weber, M. S., T. Prod'homme, S. Youssef, S. E. Dunn, C. D. Rundle, L. Lee, J. C. Patarroyo, O. Stuve, R. A. Sobel, L. Steinman, and S. S. Zamvil. 2007. Type II monocytes modulate $\mathrm{T}$ cell-mediated central nervous system autoimmune disease.

81. Jee, Y., R. Liu, X. F. Bai, D. I. Campagnolo, F. D. Shi, and T. L. Vollmer. 2006. Do Th2 cells mediate the effects of glatiramer acetate in experimental autoimmune encephalomyelitis?

82. Begum-Haque, S., A. Sharma, I. R. Kasper, D. M. Foureau, D. W. Mielcarz, A. Haque, and L. H. Kasper. 2008. Downregulation of IL-17 and IL-6 in the central nervous system by glatiramer acetate in experimental autoimmune encephalomyelitis.

83. Ruggieri, M., C. Avolio, P. Livrea, and M. Trojano. 2007. Glatiramer acetate in multiple sclerosis: a review.

84. Kim, H. J., I. Ifergan, J. P. Antel, R. Seguin, M. Duddy, Y. Lapierre, F. Jalili, and A. Bar-Or. 2004. Type 2 monocyte and microglia differentiation mediated by glatiramer acetate therapy in patients with multiple sclerosis.

85. Vieira, P. L., H. C. Heystek, J. Wormmeester, E. A. Wierenga, and M. L. Kapsenberg. 2003. Glatiramer acetate (copolymer-1, copaxone) promotes Th2 cell development and increased IL-10 production through modulation of dendritic cells.

86. Weber, M. S., R. Hohlfeld, and S. S. Zamvil. 2007. Mechanism of action of glatiramer acetate in treatment of multiple sclerosis.

87. Fridkis-Hareli, M., D. Teitelbaum, E. Gurevich, I. Pecht, C. Brautbar, O. J. Kwon, T. Brenner, R. Arnon, and M. Sela. 1994. Direct binding of myelin basic protein and synthetic copolymer 1 to class II major histocompatibility complex molecules on living antigen-presenting cells--specificity and promiscuity.

88. Fridkis-Hareli, M., D. Teitelbaum, R. Arnon, and M. Sela. 1995. Synthetic copolymer 1 and myelin basic protein do not require processing prior to binding to class II major histocompatibility complex molecules on living antigenpresenting cells.

89. Aharoni, R., D. Teitelbaum, R. Arnon, and M. Sela. 1999. Copolymer 1 acts against the immunodominant epitope $82-100$ of myelin basic protein by $\mathrm{T}$ cell receptor antagonism in addition to major histocompatibility complex blocking.

90. Stern, J. N., Z. Illes, J. Reddy, D. B. Keskin, E. Sheu, M. Fridkis-Hareli, H. Nishimura, C. F. Brosnan, L. Santambrogio, V. K. Kuchroo, and J. L. Strominger. 2004. Amelioration of proteolipid protein 139-151-induced encephalomyelitis in SJL mice by modified amino acid copolymers and their mechanisms.

91. Teitelbaum, D., R. Aharoni, R. Arnon, and M. Sela. 1988. Specific inhibition of the $\mathrm{T}$-cell response to myelin basic protein by the synthetic copolymer Cop 1 . 
92. Bettelli, E., M. Pagany, H. L. Weiner, C. Linington, R. A. Sobel, and V. K. Kuchroo. 2003. Myelin oligodendrocyte glycoprotein-specific T cell receptor transgenic mice develop spontaneous autoimmune optic neuritis.

93. Rocha, N., and J. Neefjes. 2008. MHC class II molecules on the move for successful antigen presentation.

94. Villadangos, J. A. 2001. Presentation of antigens by MHC class II molecules: getting the most out of them.

95. Ackerman, A. L., and P. Cresswell. 2004. Cellular mechanisms governing crosspresentation of exogenous antigens.

96. Monu, N., and E. S. Trombetta. 2007. Cross-talk between the endocytic pathway and the endoplasmic reticulum in cross-presentation by MHC class I molecules.

97. Di Pucchio, T., B. Chatterjee, A. Smed-Sorensen, S. Clayton, A. Palazzo, M. Montes, Y. Xue, I. Mellman, J. Banchereau, and J. E. Connolly. 2008. Direct proteasome-independent cross-presentation of viral antigen by plasmacytoid dendritic cells on major histocompatibility complex class I.

98. Burgdorf, S., C. Scholz, A. Kautz, R. Tampe, and C. Kurts. 2008. Spatial and mechanistic separation of cross-presentation and endogenous antigen presentation.

99. Keler, T., L. He, V. Ramakrishna, and B. Champion. 2007. Antibody-targeted vaccines.

100. Proft, T., and J. D. Fraser. 2003. Bacterial superantigens.

101. Proft, T., S. L. Moffatt, C. J. Berkahn, and J. D. Fraser. 1999. Identification and characterization of novel superantigens from Streptococcus pyogenes.

102. Fraser, J., V. Arcus, P. Kong, E. Baker, and T. Proft. 2000. Superantigens powerful modifiers of the immune system.

103. Petersson, K., G. Forsberg, and B. Walse. 2004. Interplay between superantigens and immunoreceptors.

104. Kamezawa, Y., T. Nakahara, S. Nakano, Y. Abe, J. Nozaki-Renard, and T. Isono. 1997. Streptococcal mitogenic exotoxin $Z$, a novel acidic superantigenic toxin produced by a $\mathrm{T} 1 \mathrm{strain}$ of Streptococcus pyogenes.

105. Proft, T., S. L. Moffatt, K. D. Weller, A. Paterson, D. Martin, and J. D. Fraser. 2000. The streptococcal superantigen SMEZ exhibits wide allelic variation, mosaic structure, and significant antigenic variation.

106. Loh, M. 2006. Superantigens as vaccine delivery vehicles for the generation of cellular immune responses, $\mathrm{PhD}$ thesis, University of Auckland, Auckland, New Zealand.

107. Gilgun-Sherki, Y., H. Panet, V. Holdengreber, R. Mosberg-Galili, and D. Offen. 2003. Axonal damage is reduced following glatiramer acetate treatment in C57/bl mice with chronic-induced experimental autoimmune encephalomyelitis.

108. Kozlov, I. A., P. C. Melnyk, K. E. Stromsborg, M. S. Chee, D. L. Barker, and C. Zhao. 2004. Efficient strategies for the conjugation of oligonucleotides to antibodies enabling highly sensitive protein detection.

109. Nimmerjahn, F., and J. V. Ravetch. 2008. Fcgamma receptors as regulators of immune responses. 
110. Teitelbaum, D., M. Fridkis-Hareli, R. Arnon, and M. Sela. 1996. Copolymer 1 inhibits chronic relapsing experimental allergic encephalomyelitis induced by proteolipid protein (PLP) peptides in mice and interferes with PLP-specific T cell responses.

111. Kayhan, B., R. Aharoni, and R. Arnon. 2003. Glatiramer acetate (Copaxone) regulates nitric oxide and related cytokine secretion in experimental autoimmune encephalomyelitis.

112. Teitelbaum, D., R. Aharoni, E. Klinger, R. Kreitman, E. Raymond, A. Malley, R. Shofti, M. Sela, and R. Arnon. 2004. Oral glatiramer acetate in experimental autoimmune encephalomyelitis: clinical and immunological studies.

113. Ruggieri, M., C. Avolio, S. Scacco, C. Pica, A. Lia, G. B. Zimatore, S. Papa, P. Livrea, and M. Trojano. 2006. Glatiramer acetate induces pro-apoptotic mechanisms involving Bcl-2, Bax and Cyt-c in peripheral lymphocytes from multiple sclerosis patients.

114. Liu, J., T. V. Johnson, J. Lin, S. H. Ramirez, T. K. Bronich, S. Caplan, Y. Persidsky, H. E. Gendelman, and J. Kipnis. 2007. T cell independent mechanism for copolymer-1-induced neuroprotection.

115. Lunde, E., K. H. Western, I. B. Rasmussen, I. Sandlie, and B. Bogen. 2002. Efficient delivery of T cell epitopes to APC by use of MHC class II-specific Troybodies.

116. Kurlander, R. J., D. M. Ellison, and J. Hall. 1984. The blockade of Fc receptormediated clearance of immune complexes in vivo by a monoclonal antibody (2.4G2) directed against Fc receptors on murine leukocytes.

117. Dickgreber, N., P. Stoitzner, Y. Bai, K. M. Price, K. J. Farrand, K. Manning, C. E. Angel, P. R. Dunbar, F. Ronchese, J. D. Fraser, B. T. Backstrom, and I. F. Hermans. 2009. Targeting antigen to MHC class II molecules promotes efficient cross-presentation and enhances immunotherapy.

118. Carayanniotis, G., and B. H. Barber. 1987. Adjuvant-free IgG responses induced with antigen coupled to antibodies against class II MHC.

119. Carayanniotis, G., and B. H. Barber. 1990. Characterization of the adjuvant-free serological response to protein antigens coupled to antibodies specific for class II MHC determinants.

120. Estrada, A., M. R. McDermott, B. J. Underdown, and D. P. Snider. 1995. Intestinal immunization of mice with antigen conjugated to anti-MHC class II antibodies.

121. Wu, J. Y., R. K. Taylor, and W. F. Wade. 2001. Anti-class II monoclonal antibody-targeted Vibrio cholerae TcpA pilin: modulation of serologic response, epitope specificity, and isotype.

122. McNeill, A. 2005. Suppression of Experimental Autoimmune Encephalomyelitis by Myelin-Specific Regulatory T Cells, PhD Thesis, University of Otago, Dunedin, New Zealand.

123. Huitinga, I., N. van Rooijen, C. J. de Groot, B. M. Uitdehaag, and C. D. Dijkstra. 1990. Suppression of experimental allergic encephalomyelitis in Lewis rats after elimination of macrophages.

124. Izikson, L., R. S. Klein, A. D. Luster, and H. L. Weiner. 2002. Targeting monocyte recruitment in CNS autoimmune disease.

125. Jung, S., I. Siglienti, O. Grauer, T. Magnus, G. Scarlato, and K. Toyka. 2004. Induction of IL-10 in rat peritoneal macrophages and dendritic cells by glatiramer acetate. 
126. Geissmann, F., C. Auffray, R. Palframan, C. Wirrig, A. Ciocca, L. Campisi, E. Narni-Mancinelli, and G. Lauvau. 2008. Blood monocytes: distinct subsets, how they relate to dendritic cells, and their possible roles in the regulation of T-cell responses.

127. Dale, D. C., L. Boxer, and W. C. Liles. 2008. The phagocytes: neutrophils and monocytes.

128. Randolph, G. J., C. Jakubzick, and C. Qu. 2008. Antigen presentation by monocytes and monocyte-derived cells.

129. Geissmann, F., S. Jung, and D. R. Littman. 2003. Blood monocytes consist of two principal subsets with distinct migratory properties.

130. Serbina, N. V., T. P. Salazar-Mather, C. A. Biron, W. A. Kuziel, and E. G. Pamer. 2003. TNF/iNOS-producing dendritic cells mediate innate immune defense against bacterial infection.

131. Sunderkotter, C., T. Nikolic, M. J. Dillon, N. Van Rooijen, M. Stehling, D. A. Drevets, and P. J. Leenen. 2004. Subpopulations of mouse blood monocytes differ in maturation stage and inflammatory response.

132. Edwards, J. P., X. Zhang, K. A. Frauwirth, and D. M. Mosser. 2006. Biochemical and functional characterization of three activated macrophage populations.

133. Zhu, B., Y. Bando, S. Xiao, K. Yang, A. C. Anderson, V. K. Kuchroo, and S. J. Khoury. 2007. CD11b+Ly-6C(hi) suppressive monocytes in experimental autoimmune encephalomyelitis.

134. Marigo, I., L. Dolcetti, P. Serafini, P. Zanovello, and V. Bronte. 2008. Tumorinduced tolerance and immune suppression by myeloid derived suppressor cells.

135. Gabrilovich, D. I., V. Bronte, S. H. Chen, M. P. Colombo, A. Ochoa, S. OstrandRosenberg, and H. Schreiber. 2007. The terminology issue for myeloid-derived suppressor cells.

136. Marhaba, R., M. Vitacolonna, D. Hildebrand, M. Baniyash, P. Freyschmidt-Paul, and M. Zoller. 2007. The importance of myeloid-derived suppressor cells in the regulation of autoimmune effector cells by a chronic contact eczema.

137. Haile, L. A., R. V. Wasielewski, J. Gamrekelashvili, C. Kruger, O. Bachmann, A. M. Westendorf, J. Buer, R. Liblau, M. P. Manns, F. Korangy, and T. F. Greten. 2008. Myeloid-Derived Suppressor Cells in Inflammatory Bowel Disease: A New Immunoregulatory Pathway.

138. Dugast, A. S., T. Haudebourg, F. Coulon, M. Heslan, F. Haspot, N. Poirier, R. Vuillefroy de Silly, C. Usal, H. Smit, B. Martinet, P. Thebault, K. Renaudin, and B. Vanhove. 2008. Myeloid-derived suppressor cells accumulate in kidney allograft tolerance and specifically suppress effector $\mathrm{T}$ cell expansion.

139. Talmadge, J. E. 2007. Pathways mediating the expansion and immunosuppressive activity of myeloid-derived suppressor cells and their relevance to cancer therapy.

140. Movahedi, K., M. Guilliams, J. Van den Bossche, R. Van den Bergh, C. Gysemans, A. Beschin, P. De Baetselier, and J. A. Van Ginderachter. 2008. Identification of discrete tumor-induced myeloid-derived suppressor cell subpopulations with distinct T cell-suppressive activity.

141. Huang, B., P. Y. Pan, Q. Li, A. I. Sato, D. E. Levy, J. Bromberg, C. M. Divino, and S. H. Chen. 2006. Gr-1+CD115+ immature myeloid suppressor cells mediate 
the development of tumor-induced T regulatory cells and T-cell anergy in tumorbearing host.

142. Burnett, R. J., C. A. Lyden, C. J. Tindal, C. M. Cave, M. N. Marra, and J. S. Solomkin. 1996. Mononuclear cell line THP-1 internalizes bactericidal/permeability-increasing protein by a non-receptor-mediated mechanism consistent with pinocytosis.

143. Youn, J. I., S. Nagaraj, M. Collazo, and D. I. Gabrilovich. 2008. Subsets of myeloid-derived suppressor cells in tumor-bearing mice.

144. Van Rooijen, N., and A. Sanders. 1994. Liposome mediated depletion of macrophages: mechanism of action, preparation of liposomes and applications.

145. Stapulionis, R., C. L. Oliveira, M. C. Gjelstrup, J. S. Pedersen, M. E. Hokland, S. V. Hoffmann, K. Poulsen, C. Jacobsen, and T. Vorup-Jensen. 2008. Structural insight into the function of myelin basic protein as a ligand for integrin alpha $\mathrm{M}$ beta 2 .

146. Strauss-Ayali, D., S. M. Conrad, and D. M. Mosser. 2007. Monocyte subpopulations and their differentiation patterns during infection.

147. Venken, K., M. Thewissen, N. Hellings, V. Somers, K. Hensen, J. L. Rummens, and P. Stinissen. 2007. A CFSE based assay for measuring CD4+CD25+ regulatory $\mathrm{T}$ cell mediated suppression of auto-antigen specific and polyclonal $\mathrm{T}$ cell responses.

148. Mannering, S. I., J. S. Morris, K. P. Jensen, A. W. Purcell, M. C. Honeyman, P. M. van Endert, and L. C. Harrison. 2003. A sensitive method for detecting proliferation of rare autoantigen-specific human T cells.

149. Kanai, T., K. Uraushihara, T. Totsuka, Y. Nemoto, R. Fujii, T. Kawamura, S. Makita, D. Sawada, H. Yagita, K. Okumura, and M. Watanabe. 2006. Ameliorating effect of saporin-conjugated anti-CD11b monoclonal antibody in a murine T-cell-mediated chronic colitis.

150. Schlecht, G., J. Loucka, H. Najar, P. Sebo, and C. Leclerc. 2004. Antigen targeting to $\mathrm{CD} 11 \mathrm{~b}$ allows efficient presentation of CD4+ and CD8+ T cell epitopes and in vivo Th1-polarized T cell priming.

151. Cote, C. K., K. M. Rea, S. L. Norris, N. van Rooijen, and S. L. Welkos. 2004. The use of a model of in vivo macrophage depletion to study the role of macrophages during infection with Bacillus anthracis spores.

152. Van Rooijen, N., and A. Sanders. 1996. Kupffer cell depletion by liposomedelivered drugs: comparative activity of intracellular clodronate, propamidine, and ethylenediaminetetraacetic acid.

153. Bonifaz, L., D. Bonnyay, K. Mahnke, M. Rivera, M. C. Nussenzweig, and R. M. Steinman. 2002. Efficient targeting of protein antigen to the dendritic cell receptor DEC-205 in the steady state leads to antigen presentation on major histocompatibility complex class I products and peripheral CD8+ T cell tolerance.

154. Anderson, C. F., and D. M. Mosser. 2002. Cutting edge: biasing immune responses by directing antigen to macrophage Fc gamma receptors.

155. van Bergen, J., F. Ossendorp, R. Jordens, A. M. Mommaas, J. W. Drijfhout, and F. Koning. 1999. Get into the groove! Targeting antigens to MHC class II. 
156. Dickgreber, N., Stoitzner, P., Bai, Y., Price, K.M., Farrand, K.J., Ronchese, F., Fraser, J.D., Backstrom, B.T., Hermans, I.F. 2009. Targeting Antigen to MHC Class II Molecules Promotes Efficient Cross-Presentation and Enhances Immnuotherapy.

157. Assudani, D. P., R. B. Horton, M. G. Mathieu, S. E. McArdle, and R. C. Rees. 2007. The role of CD4+ T cell help in cancer immunity and the formulation of novel cancer vaccines.

158. Chen, M., R. M. Valenzuela, and S. Dhib-Jalbut. 2003. Glatiramer acetatereactive $\mathrm{T}$ cells produce brain-derived neurotrophic factor.

159. Hawiger, D., R. F. Masilamani, E. Bettelli, V. K. Kuchroo, and M. C. Nussenzweig. 2004. Immunological unresponsiveness characterized by increased expression of CD5 on peripheral T cells induced by dendritic cells in vivo.

160. Saresella, M., I. Marventano, R. Longhi, F. Lissoni, D. Trabattoni, L. Mendozzi, D. Caputo, and M. Clerici. 2008. CD4+CD25+FoxP3+PD1- regulatory T cells in acute and stable relapsing-remitting multiple sclerosis and their modulation by therapy.

161. Sanna, A., M. L. Fois, G. Arru, Y. M. Huang, H. Link, M. Pugliatti, G. Rosati, and S. Sotgiu. 2006. Glatiramer acetate reduces lymphocyte proliferation and enhances IL-5 and IL-13 production through modulation of monocyte-derived dendritic cells in multiple sclerosis.

162. Weber, M. S., M. Starck, S. Wagenpfeil, E. Meinl, R. Hohlfeld, and C. Farina. 2004. Multiple sclerosis: glatiramer acetate inhibits monocyte reactivity in vitro and in vivo.

163. Li, Q., R. Milo, H. Panitch, P. Swoveland, and C. T. Bever, Jr. 1998. Glatiramer acetate blocks the activation of THP-1 cells by interferon-gamma.

164. Brenner, T., S. Brocke, F. Szafer, R. A. Sobel, J. F. Parkinson, D. H. Perez, and L. Steinman. 1997. Inhibition of nitric oxide synthase for treatment of experimental autoimmune encephalomyelitis.

165. Iarlori, C., D. Gambi, A. Lugaresi, A. Patruno, M. Felaco, M. Salvatore, L. Speranza, and M. Reale. 2008. Reduction of free radicals in multiple sclerosis: effect of glatiramer acetate (Copaxone). 


\section{APPENDIX 1:}

\section{SUPPLEMENTARY FIGURES AND \\ TABLES}




\section{A1.1: GA does not protect 2D2 mice from EAE induced with $25 \mu \mathrm{g} \mathrm{MOG}_{35-55}$.}

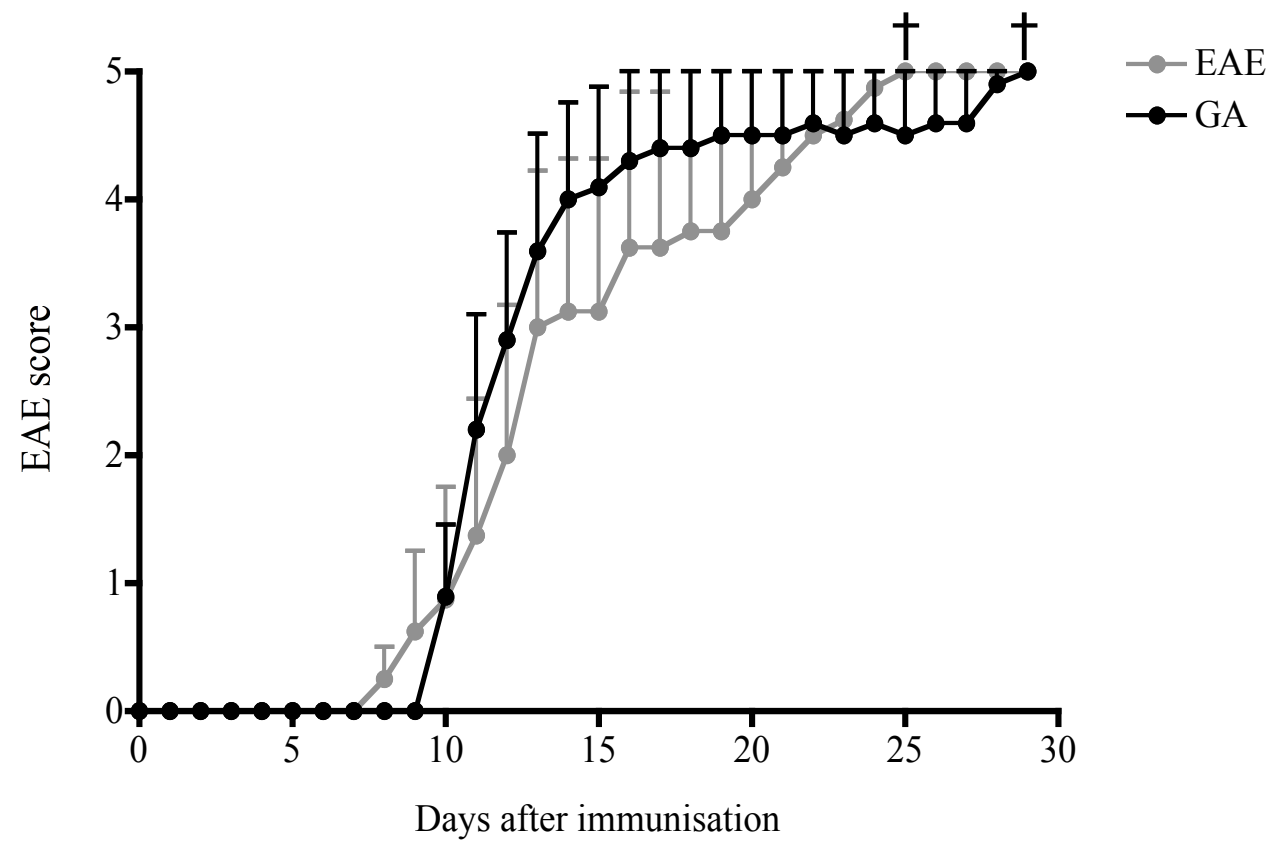

EAE was induced in 2D2 mice with $25 \mu \mathrm{g} \mathrm{MOG}_{35-55}$. Mice were either left untreated (EAE) or co-immunised with $500 \mu \mathrm{g} \mathrm{GA}$. ( $\mathrm{p}>0.05$ for EAE vs. GA; mean $\pm \mathrm{SEM}$ of $\mathrm{n}=$ 5 mice per group). $\dagger$ indicates that all mice in the corresponding group were euthanised due to severe EAE. 


\section{A1.2: Optimisation of the Conjugation of GA to Modified SAgs.}

GA was incubated with varying molar ratios of SMCCplus as described in Materials and Methods in detail. Excess chemical was removed and activated GA was mixed with the reduced SAg in different ratios, and incubated at varying conditions. Efficacy of conjugation was assessed by non-reducing SDS-PAGE. Representative SDS gel images of "poor", "fair" and "good" conjugation reactions are shown in A1.3.

\begin{tabular}{|c|c|c|c|}
\hline Ratio SAg:GA & Ratio GA:SMCCplus & Incubation & Outcome \\
\hline $1: 1$ & $1: 2$ & ON at $4{ }^{\circ} \mathrm{C}$ & Poor conjugation \\
\hline $1: 1$ & $1: 2$ & $1 \mathrm{~h}$ at $\mathrm{RT}$ & Poor conjugation \\
\hline $1: 5$ & $1: 2$ & $\mathrm{ON}$ at $4{ }^{\circ} \mathrm{C}$ & Fair conjugation \\
\hline $1: 5$ & $1: 2$ & 1 h at $\mathrm{RT}$ & Fair conjugation \\
\hline $1: 10$ & $1: 2$ & $\mathrm{ON}$ at $4{ }^{\circ} \mathrm{C}$ & Fair conjugation \\
\hline $1: 20$ & $1: 2$ & $\mathrm{ON}$ at $4^{\circ} \mathrm{C}$ & Fair conjugation \\
\hline $1: 1$ & $1: 10$ & $\mathrm{ON}$ at $4^{\circ} \mathrm{C}$ & Poor conjugation \\
\hline $1: 5$ & $1: 10$ & $\mathrm{ON}$ at $4^{\circ} \mathrm{C}$ & Fair conjugation \\
\hline $1: 20$ & $1: 10$ & $\mathrm{ON}$ at $4^{\circ} \mathrm{C}$ & Poor conjugation \\
\hline $1: 5$ & $1: 5$ & $\mathrm{ON}$ at $4{ }^{\circ} \mathrm{C}$ & Good conjugation \\
\hline $1: 10$ & $1: 5$ & $\mathrm{ON}$ at $4{ }^{\circ} \mathrm{C}$ & Good conjugation \\
\hline $1: 20$ & $1: 5$ & $\mathrm{ON}$ at $4{ }^{\circ} \mathrm{C}$ & Fair conjugation \\
\hline
\end{tabular}


A1.3: Optimisation of the Conjugation Reaction of GA to Modified SAgs.

\begin{tabular}{|c|c|}
\hline Ratio GA:SMCCplus & \\
\hline Ratio SAg:GA & \\
\hline Efficacy of conjugation & \\
\hline Loading controls & SAg (ox) \\
\hline
\end{tabular}

The effect of varying reaction conditions on the efficacy of conjugation of GA to modified SAg was evaluated. GA was conjugated to SAg under varying conditions (see A1.2), and efficiency of conjugation was assessed by non-reducing SDS-PAGE and Coomassie blue staining. SAg was loaded onto the gel alone before (ox) and after (red) reduction as control. MWM: molecular weight marker. 


\section{A1.4: GA does not bind to MHCII expressing cells in vitro.}

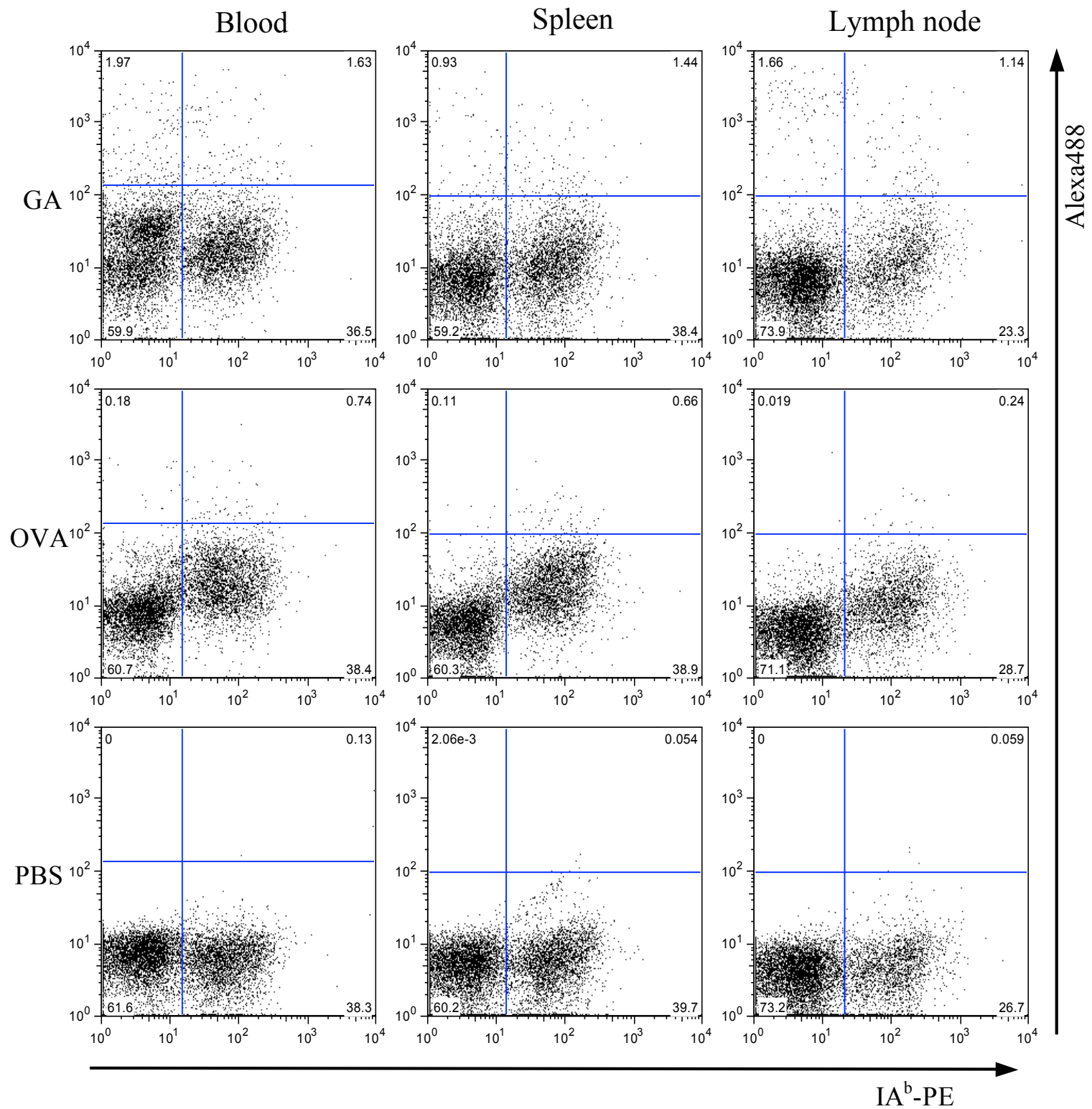

Single cell suspensions were prepared from spleens, inguinal lymph nodes and blood of C57BL/6J mice. The cells were incubated in absence (PBS) or presence of $10 \mu \mathrm{g} / \mathrm{ml}$ Alexa488 labelled GA or OVA for one hour at $37{ }^{\circ} \mathrm{C}$ in a 96 -well plate at $5 \times 10^{4}$ cells per well. The cells were then stained for cell surface markers and analysed by flow cytometry. Data represents one out of two independent experiments with duplicate wells. 
A1.5: $\mathrm{GA}^{+}$cells in the blood of MHCII deficient mice show monocyte phenotype.
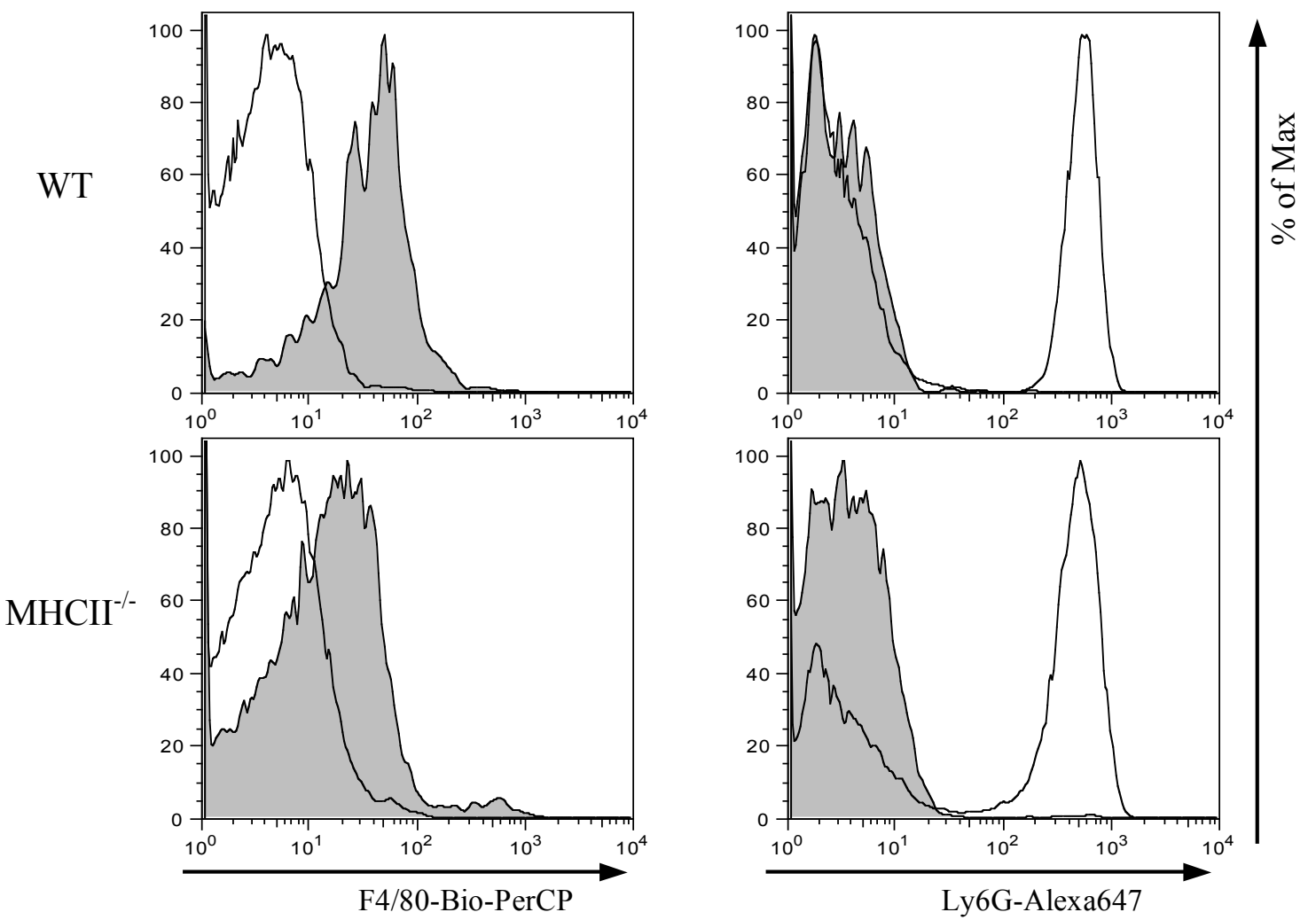

$\mathrm{C} 57 \mathrm{BL} / 6 \mathrm{~J}(\mathrm{WT})$ or $\mathrm{B} \mathrm{Aa}^{0} / \mathrm{Aa}^{0}\left(\mathrm{MHCII}^{-/}\right)$mice received intravenous injections of $50 \mu \mathrm{g}$ GA-Alexa488. Blood cells were recovered one hour after the injections and analysed by flow cytometry. CD11 $\mathrm{b}^{\text {hi }}$ cells that showed GA staining (shaded histograms) were $\mathrm{F} 4 / 80^{\text {lo }}$ and Ly6G'. Open histograms represent $\mathrm{CD} 11 \mathrm{~b}^{\text {hi }}$ cells that did not stain for GA (F4/80- $\mathrm{Ly}^{-} \mathrm{G}^{+}$ granulocytes). Histograms are gated on $\mathrm{CD} 11 \mathrm{~b}^{\text {hi }}$ cells. Data represents one out of two independent experiments. 


\section{A1.6: Co-immunisation treatment with trypsinogen suppresses EAE.}

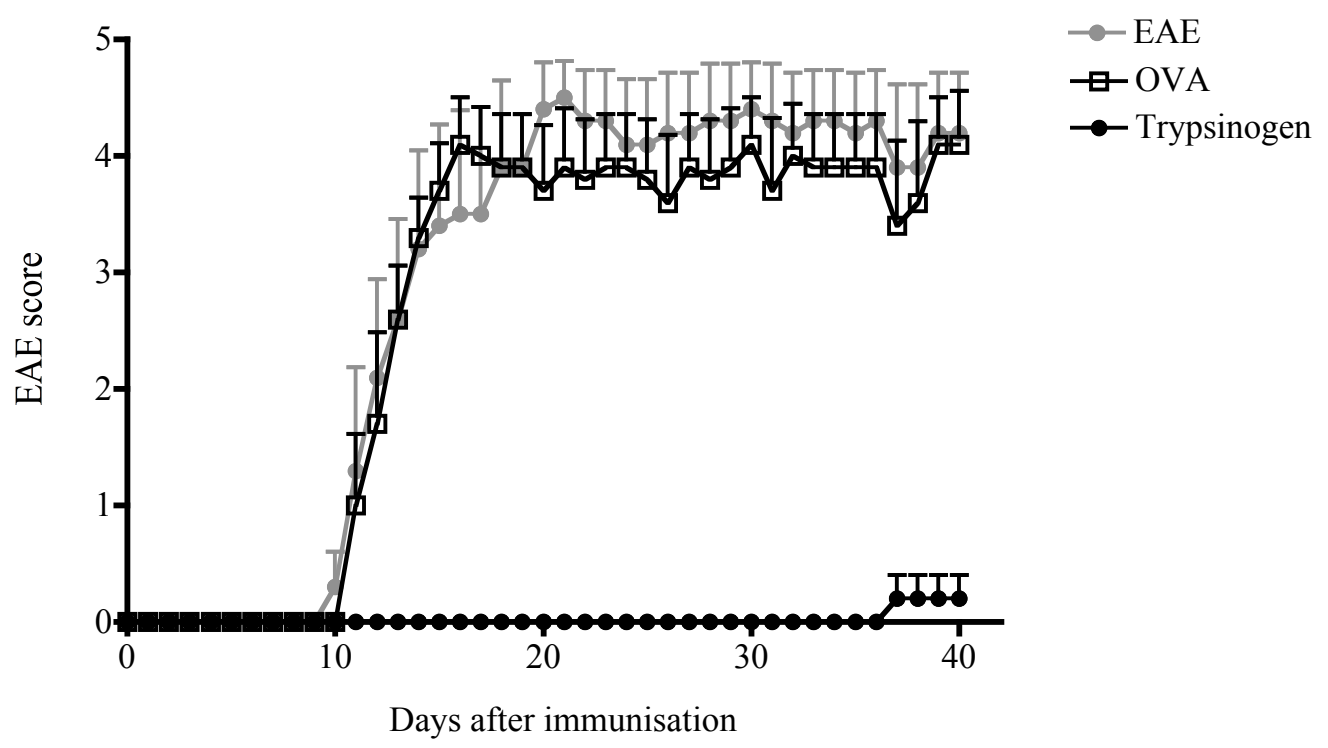

EAE was induced in C57BL/6J mice, and the mice were either left untreated or treated by co-immunisation with $500 \mu \mathrm{g}$ trypsinogen or $500 \mu \mathrm{g}$ OVA. ( $<<0.001$ for EAE vs. Trypsinogen, $\mathrm{p}>0.05$ for EAE vs. OVA; mean \pm SEM of $n=5$ mice per group). Data represents one out of at least two independent experiments. 


\section{A1.7: Depletion of $\mathrm{CD25}^{+}$cells diminishes GA treatment.}

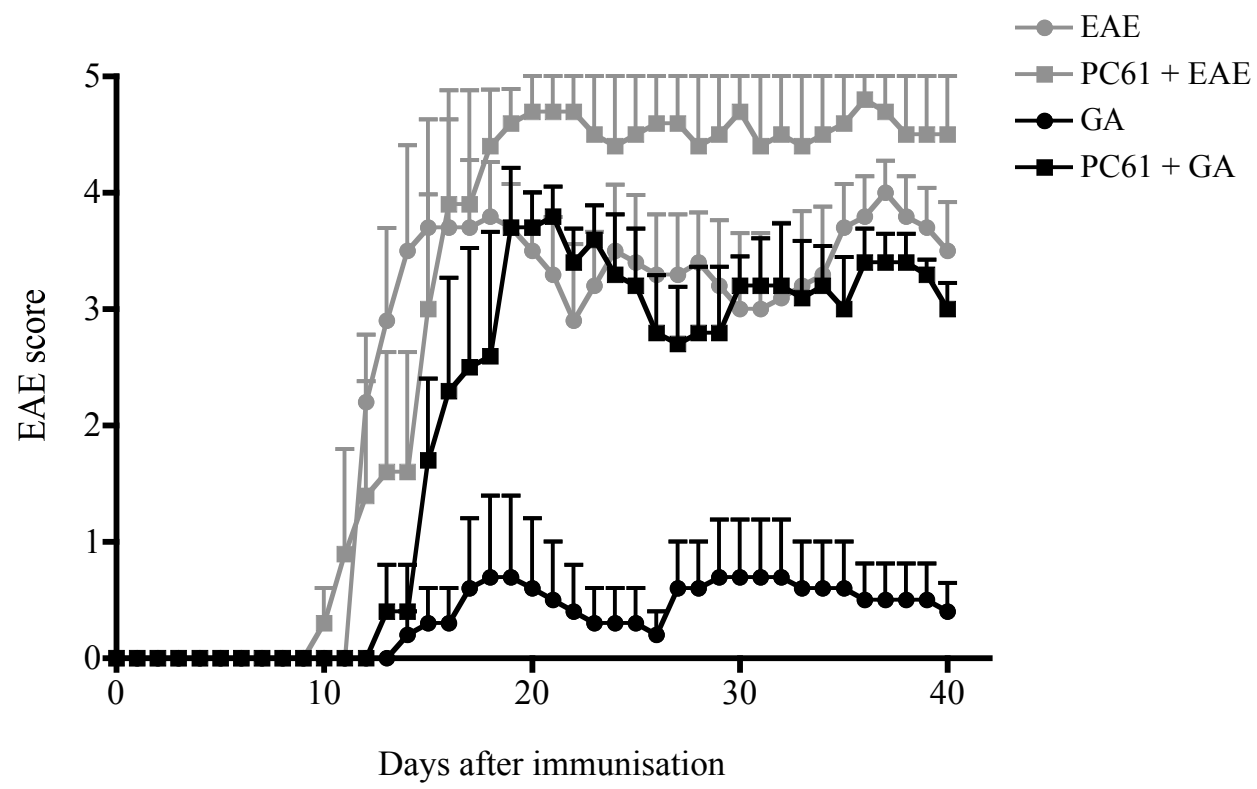

$\mathrm{CD} 25^{+}$cells were depleted in $\mathrm{C} 57 \mathrm{BL} / 6 \mathrm{~J}$ mice through intraperitoneal injections of $200 \mu \mathrm{g}$ anti-CD25 antibody (clone PC61) in PBS three days before EAE induction. Mice were treated by co-immunisation with $500 \mu \mathrm{g}$ GA. ( $<<0.05$ for EAE vs. PC61 + EAE, $\mathrm{p}<0.001$ for GA vs. PC61 $+\mathrm{GA}$; mean \pm SEM of $\mathrm{n}=5$ mice per group). Data represents one out of two independent experiments. 


\section{Acknowledgements}

First of all, I wish to express my sincere gratitude to my family and friends I left behind, for supporting me in my endeavour other end of the world, and for showing no hard feelings because I went as far away from them as humanly possible.

I sincerely thank my primary supervisor Thomas Bäckström for his dedicated supervision and support.

Most sincere thanks to Jacquie, for taking over the supervision of my project at a crucial stage and invaluable advice and support, as well as critical revision of the manuscript of this thesis.

I thank Evelyn, Liz, Franca, Anne, Kylie, Nina and Willy-John for their valuable advice or assistance. I am grateful to Anne and Liz for their critical comments and suggestions on the manuscript.

Thanks to everyone at the Malaghan Institute for an unbeatable working atmosphere.

In addition, I thank Tommy, so that he doesn't feel left out. And buys me a beer.

Thanks to Patricia for great organisation, always having some time for troubleshooting and having the right advice at ready every time asked for.

I thank Nicole, Aaron, Emilie, Jérémie, Jaro, Pavla, Tim and Joanna, for making Wellington an even better place to live.

I am especially thankful to Clare for teaching me almost every immunological method I know, for fruitful discussions, mutual encouragement and support. 